\title{
QUANTITATIVE INTERFEROMETRIC MEASUREMENTS OF THE REFRACTIVE INDEX AND ITS APPLICATIONS
}

\author{
By \\ Mohammed Yahya \\ Bachelor of Science, Mosul University, 1988 \\ Master of Science, Molecular and Nuclear Physics, Mosul University, 1994 \\ Master of Science, Biomedical Physics, Ryerson University, 2010
}

\author{
A dissertation presented to \\ Ryerson University \\ in partial fulfillment of the requirement \\ for the degree of \\ Doctor of Philosophy \\ In the program of \\ Mechanical Engineering
}

Toronto, Ontario, Canada, 2016

(C) Mohammed Yahya, 2016 


\section{Author's Declaration}

I hereby declare that I am the sole author of this dissertation. This is a true copy of the dissertation, including any required final revisions, as accepted by my examiners.

I authorize Ryerson University to lend this dissertation to other institutions or individuals for the purpose of scholarly research.

I further authorize Ryerson University to reproduce this dissertation by photocopying or by other means, in total or in part, at the request of other institutions or individuals for the purpose of scholarly research.

I understand that my dissertation may be made electronically available to the public.

\section{Yahya}




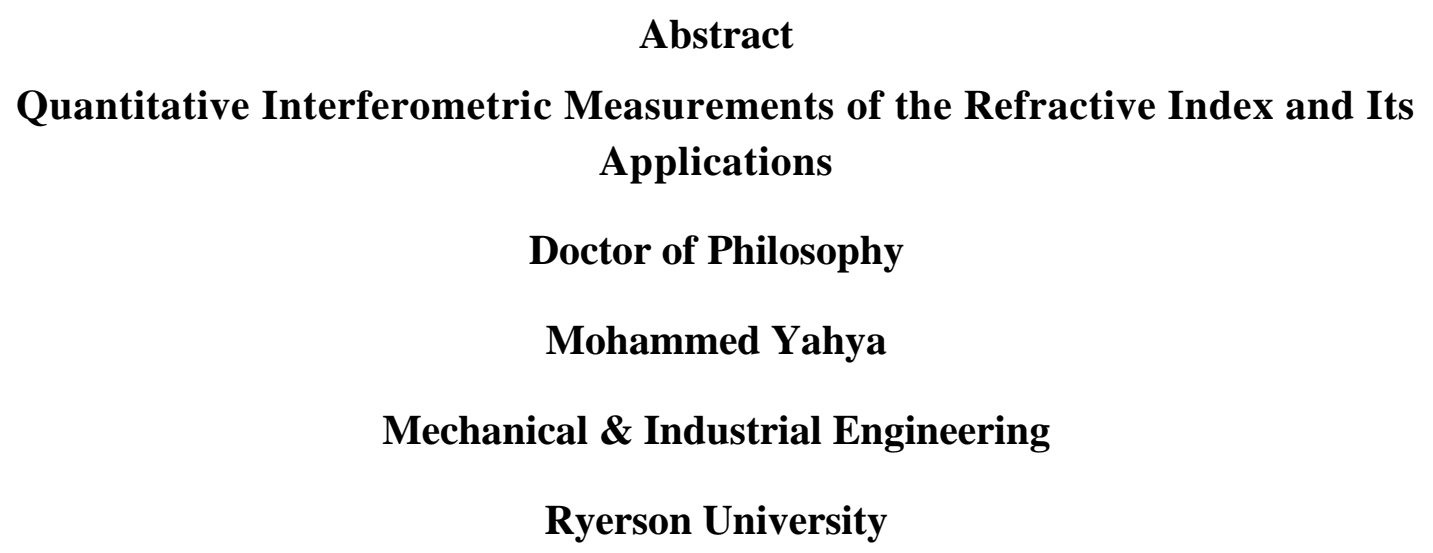

2016

Optical interferometry is a non-contact method used for the imaging and measurement of the optical properties of small or large objects with nanoscale accuracy. Interferometers are essential optical systems in engineering and medical science and are generally used to measure refractive index changes, cell pathology and shape irregularities. The ability to provide quantitative, noninvasive measurements makes interferometers suitable candidates for the study of living cells in vivo and in vitro applications.

In this study, a simple interferometric system capable of providing precise measurements of the refractive indices of transparent and semi-transparent mediums was designed and developed based on the Mach-Zehnder arrangement. During the development phase, the system was used to take precise measurements of the contrast factors of the three hydrocarbon components $1,2,3,4$ Tetrtahydronaphtalenene (THN), Isobutylbenzen (IBB), and Dodecane $\left(n \mathrm{C}_{12}\right)$, prepared in the laboratory. The results were also compared with the corresponding results obtained using the Abbemat refractometer. The results were in agreement with those obtained from both techniques as well as the results obtained from the literature, confirming the accuracy of the measurements obtained with the new system with deviations of $\pm 2.50 \times 10^{-3}$.

Blood analysis is a routine procedure used in medical diagnostics to confirm a patient's condition. Measuring the optical properties of blood is difficult due to the non-homogenous nature of the blood itself. In addition, there is a lot of variation in the refractive indices reported in the literature. These are the reasons that motivated this research to develop an empirical model that can be used to predict the refractive index of human blood as a function of concentration, temperature and wavelength. The experimental measurements were conducted on mimicking 
phantom, that shows better stability compared to human blood, using the Abbemat Refractometer. The values obtained using the model was in substantial agreement with those obtained experimentally with standard deviations of $\pm 2.33 \times 10^{-3}$.

Once the accuracy of the interferometric system was confirmed, the system was used to study the refractive index and morphology of human red blood cells. The results of the analysis confirmed the system's ability to determine refractive index and/or blood hematocrit values with appropriate clinical accuracy. 


\section{Acknowledgments}

First and foremost, I would like to express my gratitude and deep appreciation to my dear wife Sura, who has been with me all these years. Thank you for your steadfast love, support, encouragement and patience. You have kept me going through the most difficult times and have made them the best years of my life.

I would like to express my gratitude and sincere thanks to my supervisor Dr. Z. Saghir for giving me the opportunity to work under his supervision. Thank you for your patience, guidance, encouragement and friendship during my graduate studies at Ryerson University.

I would also like to thank my doctoral committee members for their encouragement, accessibility, insightful comments, valuable discussions and difficult questions.

I am very grateful for Dr. J. Alirezaie and Dr. Y. Xu, who supported me during the most difficult year of my studies.

I would like to thank my friends and fellow lab mates in the mechanical engineering department at Ryerson for the stimulating discussions and all of the great times we have shared in the last four years.

I gratefully acknowledge Dr. T. Yousefi and Dr. Md Abdur Rahman for their time and valuable feedback on a preliminary version of this thesis. I am deeply thankful to Dr. A. Ahadi for his valuable input in my research. Great thanks to my physician Dr. N. Mati and my dear friend Ms. K. Lavoie for their supports and help.

I am deeply thankful to my family for their love, support, sacrifices and encouragement. To my sister Dr. A. Yahya, I could not have done this without you.

To my dear sons Zain and Zaid, thank you boys for keeping daddy extra busy during these years. Thank you, Sarah, for being my fun. 


\section{Table of Contents}

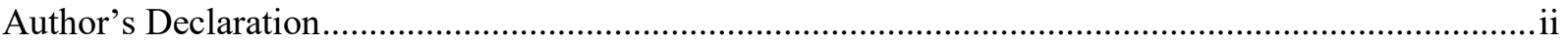

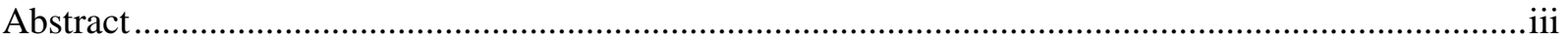

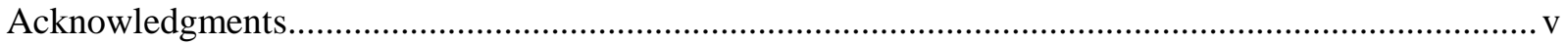

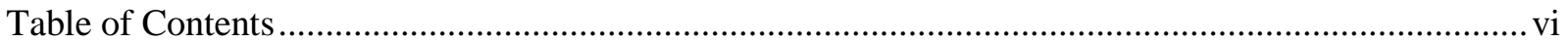

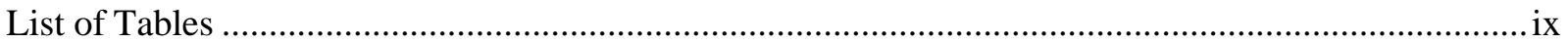

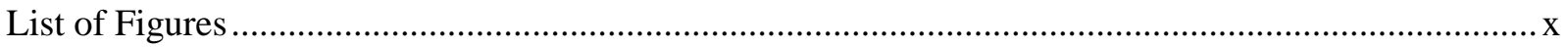

List of Symbols and Abbreviations...............................................................................................

CHAPTER 1 RESEARCH MOTIVATION AND LITERATURE REVIEW ........................................ 1

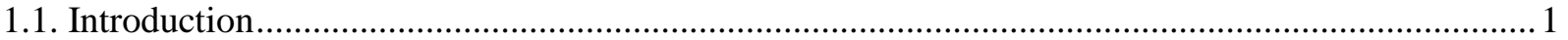

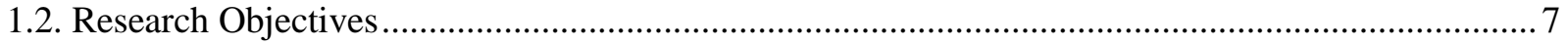

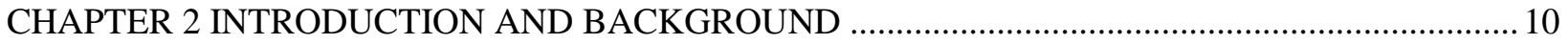

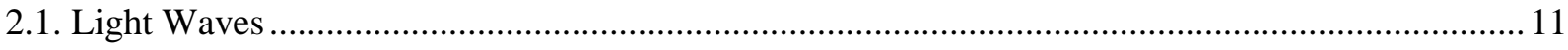

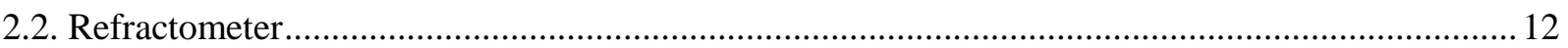

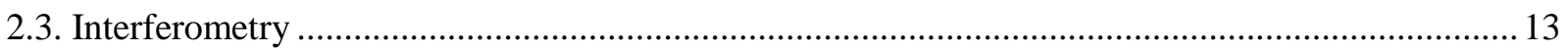

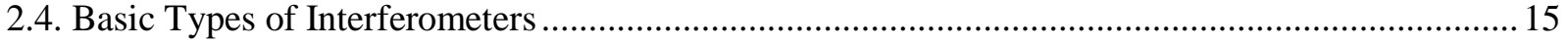

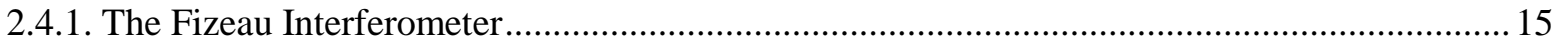

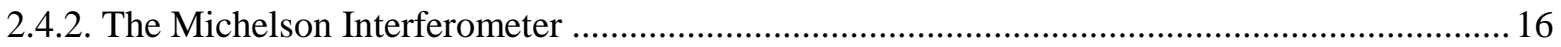

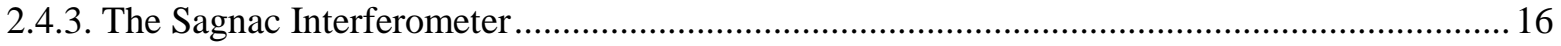

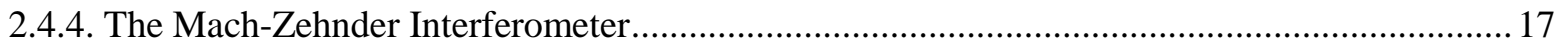

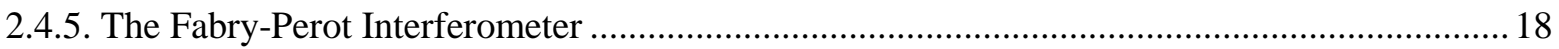

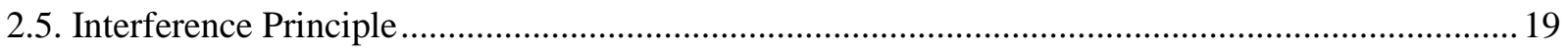

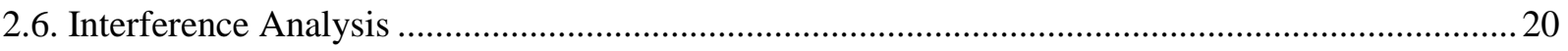

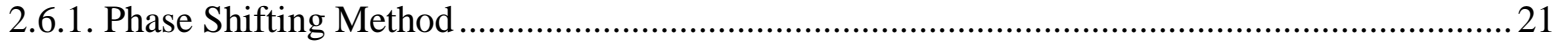

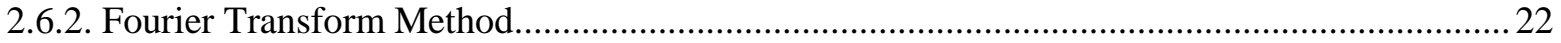

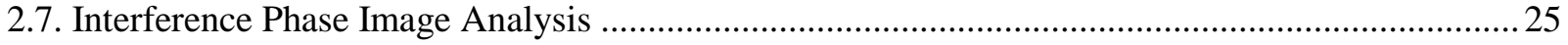

CHAPTER 3 PREDICTION AND EXPERIMENTAL MEASUREMENT OF REFRACTIVE INDEX IN

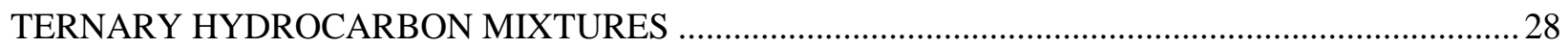

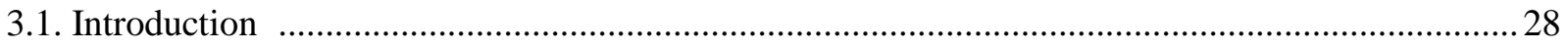

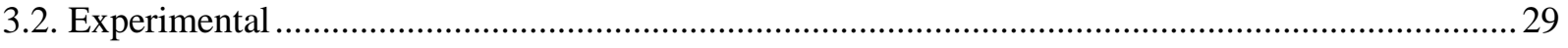

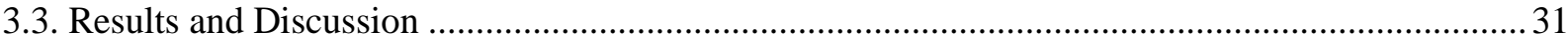




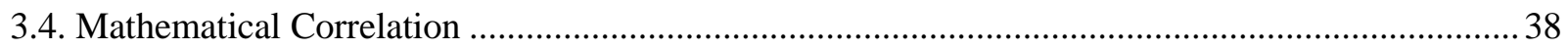

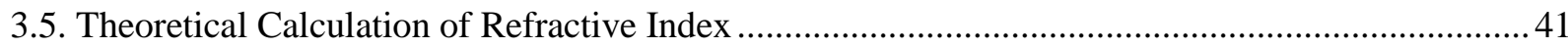

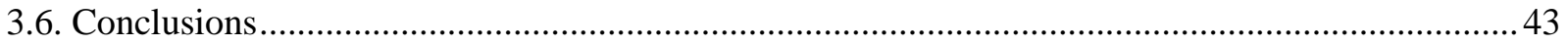

CHAPTER 4 MEASUREMENT OF OPTICAL CONTRAST FACTORS IN TERNARY

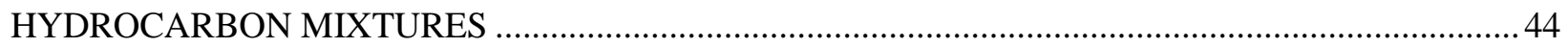

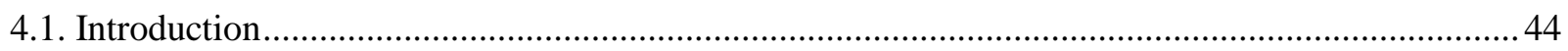

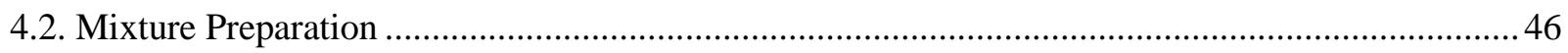

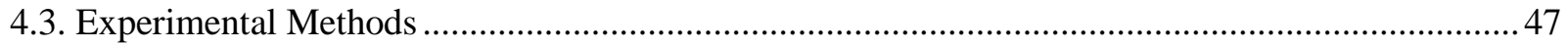

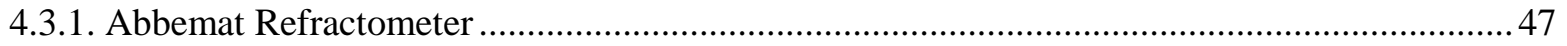

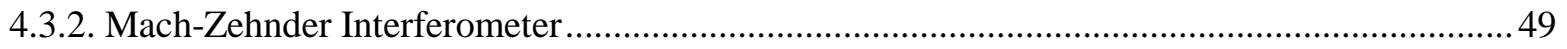

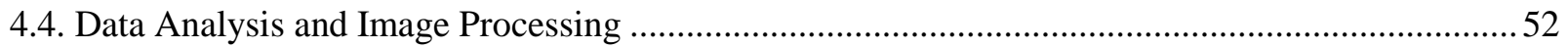

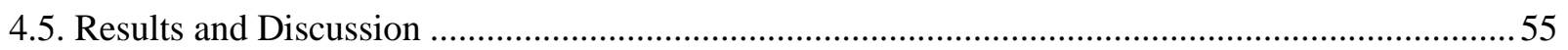

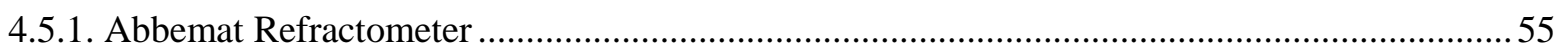

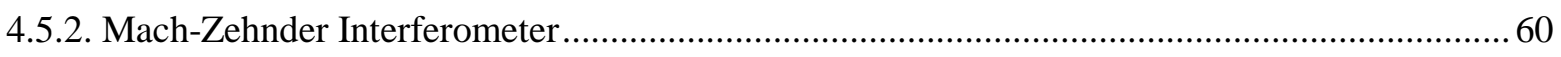

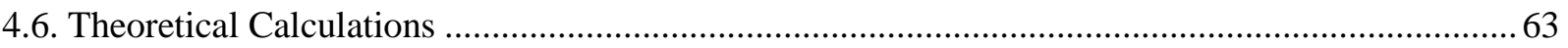

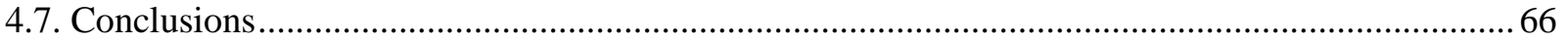

CHAPTER 5 EMPIRICAL MODELLING TO PREDICT THE REFRACTIVE INDEX OF HUMAN

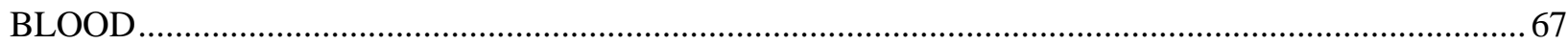

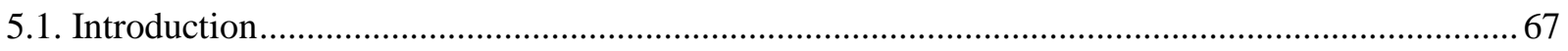

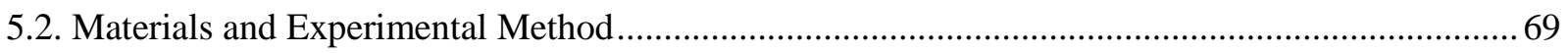

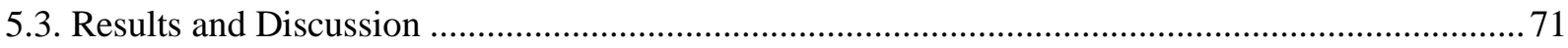

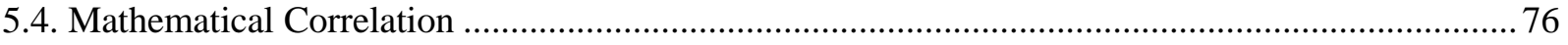

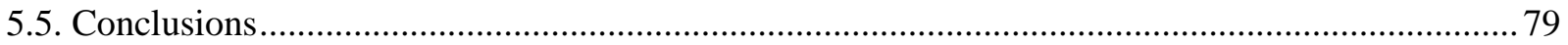

CHAPTER 6 QUANTITATIVE INTERFEROMETRIC MEASUREMENTS OF THE REFRACTIVE

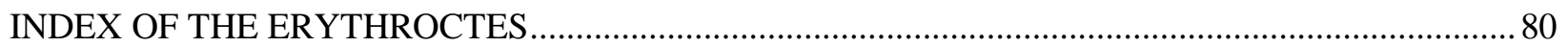

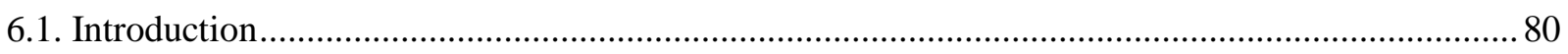

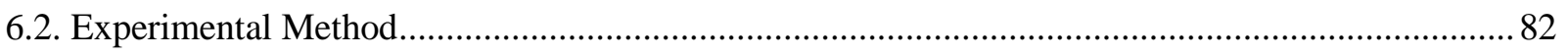

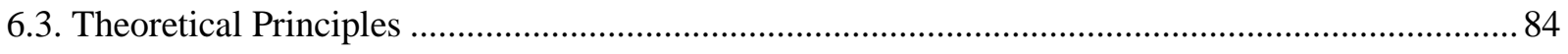

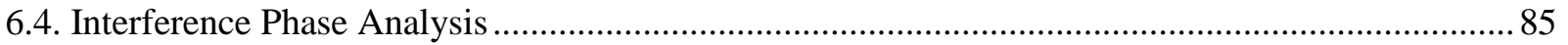

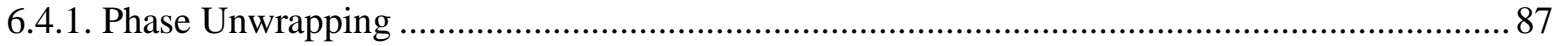

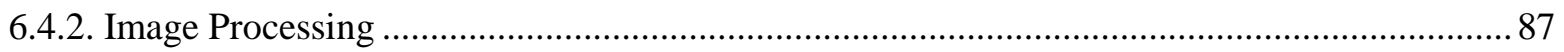

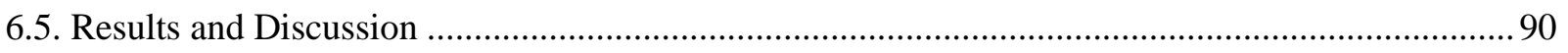

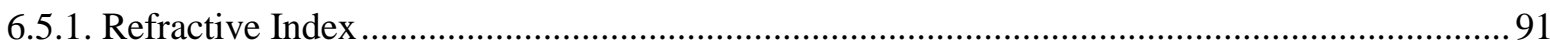

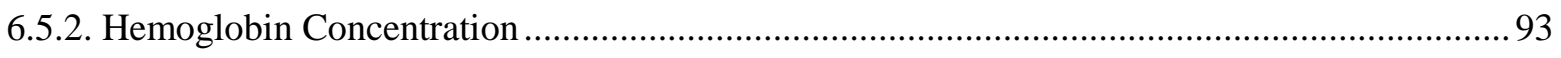




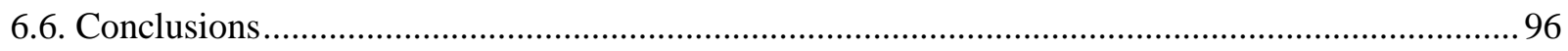

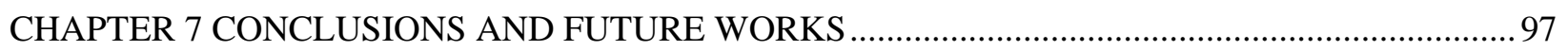

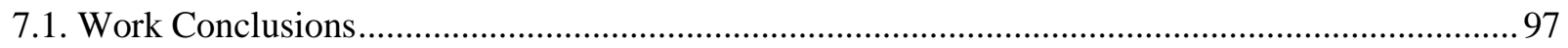

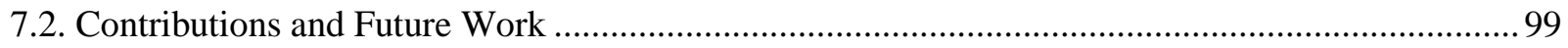

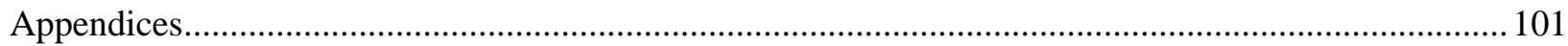

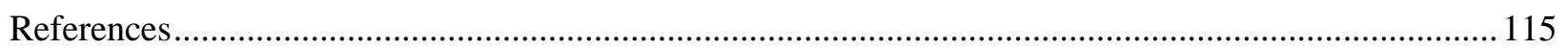




\section{List of Tables}

Table 3.1: Refractive indices and temperature coefficients of $\mathrm{THN}, \mathrm{IBB}$, and $n \mathrm{C}_{12}$ at temperatures of $\mathrm{T}=(293.15$ to 318.15$) \mathrm{K}$ and wavelength of $657.2 \mathrm{~nm}$ at atmospheric pressure $\mathrm{P}=0.1 \mathrm{MPa}^{\mathrm{a}}$. 32

Table 3.2: The estimated coefficients of the proposed correlation were obtained using a multiinput regression analysis and an analysis of variance ANOVA using MatLab®. 40

Table 3.3: Standard deviations of the experimental results in this study and its corresponding found in literature.

Table 4.1: The concentration dependence of the refractive index of using the Abbemat refractometer at a temperature $298.15 \mathrm{~K}$ for different wavelengths. a- for the (0.501-0.110-0.389) system and $\mathrm{b}$ - for the $(0.441-0.217-0.342)$ system at atmospheric pressure $\mathrm{P}=0.1 \mathrm{MPa}{ }^{*}$.

Table 4.2: Calculated concentration and temperature coefficients of refractive indices of the two investigated ternary systems of THN-IBB- $n C_{12}$ : a- $(0.501-0.110-0.389)$ and b- $(0.441-0.217-$ 0.342 ) at atmospheric pressure $\mathrm{P}=0.1 \mathrm{MPa}$.

Table 4.3: Refractive index temperature coefficient of at different compositions of (nTHN)IBB , and (nIBB)THN at a wavelength of $632.9 \mathrm{~nm}$ conducted by refractometer and MZI with relative error for the ternary systems of: a- (0.501-0.110-0.389) and b- (0.441-0.217-0.342)

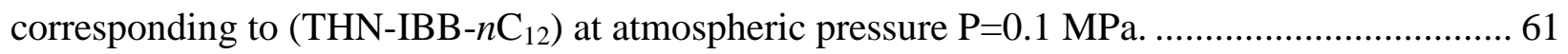
Table 4.4: Refractive index concentration coefficient of at different compositions of (nTHN)IBB , and (nIBB)THN at a wavelength of $632.9 \mathrm{~nm}$ conducted by refractometer and MZI with relative error for the ternary systems of: $a-(0.501-0.110-0.389)$ and b- (0.441-0.2170.342 ) corresponding to (THN-IBB- $n \mathrm{C}_{12}$ ).

Table 5.1: The real part of the refractive index of human blood mimicking phantom for various concentrations $\mathrm{C}=(0$ to 150$) \mathrm{g} / \mathrm{l}$ at temperatures of $\mathrm{T}=(293.15$ to 318.15$) \mathrm{K}$ and wavelengths of $\lambda=(657.2$ to 436$) \mathrm{nm}$. The table also presents the specific refraction concentrations and temperature increments $\alpha, \beta$ respectively..................................................................... 71

Table 6.1: Comparison results between the manual and the automated cells count.....................................................................94 


\section{List of Figures}

Figure 1-1: Whole human blood showing the red blood cells, platelets and the different white blood cells. .................................................................................................................. 4

Figure 2-1: Schematic diagram of a Refractometer illustrating, a-The Incident light waves and the critical angle and $b$ - The working principle of the refractometer. .................................... 13 Figure 2-2: Interference can be formed by the superposition (addition) of two traveling waves, of equal amplitude and frequency forming, fringe pattern known as interferogram. .................... 14 Figure 2-3: A Fizeau interferometer arrangement whereby two reflecting surfaces are placed

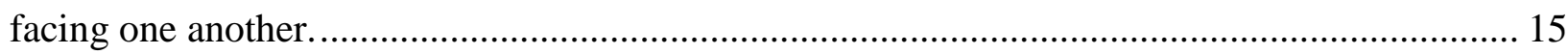

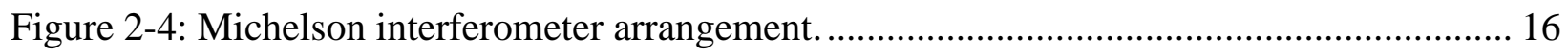

Figure 2-5: Sagnac Interferometer arrangement. .......................................................... 17

Figure 2-6: Schematic diagram of a Mach-Zehnder interferometer. ..................................... 18

Figure 2-7: Fabry-Perot Interferometer arrangement. ..................................................... 19

Figure 2-8: Schematic of the amplitude and intensity calculations occur in one interference cycle....................................................................20

Figure 2-9: a- Fourier spectra image and b- Separated Fourier spectra signal of one fringe pattern. ............................................................................................................ 23

Figure 3-1: Refractive index of (nTHN)IBB|nC12 at wavelengths $657.2 \mathrm{~nm}$ and $\mathrm{T}=298.15 \mathrm{~K}$ as function of ternary system composition........................................................................ 32 Figure 3-2: Concentration variation of refractive indices of the THN-IBB-nC12 mixtures at $\mathrm{T}=$ $298.15 \mathrm{~K}$ with respect to mass fraction over the entire concentration, plotted at different

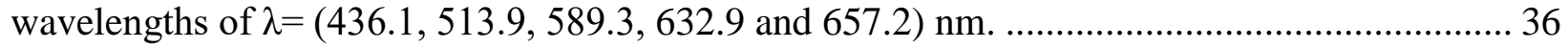
Figure 3-3: Temperature coefficients of refractive indices of the THN-IBB-nC12 mixtures at $\mathrm{T}=$ $298.15 \mathrm{~K}$ with respect to mass fraction over the entire concentration, plotted at different

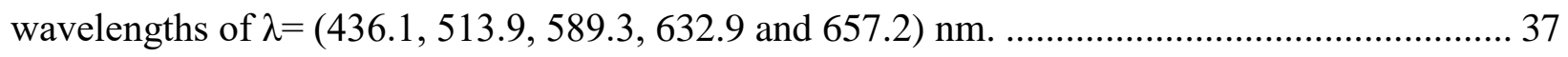
Figure 3-4: Refractive index with respect to the wavelength of the ternary mixture (nIBB)nC12 $\mid \mathrm{THN}=0.1$, at $\mathrm{T}=298.15 \mathrm{~K}$ and different concentrations of IBB, --- is the Cauchy formula. 38

Figure 3-5: a- Relative error of the experimental refractive index values with the predicted, calculated and estimated values using mixing rules for various compositions of the (nIBB)nC12 $\mid \mathrm{THN}$ at $\mathrm{T}=298.15 \mathrm{~K}$ at a wavelength $0 \mathrm{f} 657.2 \mathrm{~nm}$. b- Relative error of the same mixture at different IBB concentration........................................................................ 42 Figure 4-1: Schematic drawing of the Mach-Zehnder Interferometer setup used in this study. .. 49 Figure 4-2: Schematic drawing of the cell holder used in the Mach-Zehnder interferometer: AThe classic cell holder used in the literature, B- the cell holder developed by the authors and used in this study. 50

Figure 4-3: Interferogram sample show the fringes quality and the temperature distribution: AFingers obtained using the classic cell holder found in the literature, B- Fingers obtained using 
the developed cell holder showing a proper and accurate temperature distribution patterns along the examined mixtures. 51

Figure 4-4: The refractive index of the two investigated systems: (0.501-0.110-0.389) and (0.441-0.217-0.342) corresponding to THN-IBB-nC12 molar fraction. 58

Figure 4-5: Concentration derivative of refractive indices for various compassions of (nTHN)n C12|IBB at a single temperature of $298 \mathrm{~K}$ with respect to the two investigated systems: A(0.501-0.110-0.389) and B- (0.441-0.217-0.342) corrosponding to THN-IBB-nC12 ............... 58 Figure 4-6: Relative error of the experimental and the estimated values of refractive index obtained with mixing rules for various compositions of (nTHN)nC12|IBB at a single temperature of $298 \mathrm{~K}$ with respect to the two investigated systems: A- $(0.501-0.110-0.389)$ and B- $(0.441-$ 0.217-0.342) corrosponding to THN-IBB-nC12.

Figure 5-1: Carve fitting plot showing the refractive index as a function of a- temperature and concentration, b- wavelength and concentration. 75

Figure 5-2: Predicted values of the real part of the refractive index of $\mathrm{Hb}$ mimicking phantom solutions at the concentration of 355.5 and $0 \mathrm{~g} / \mathrm{l}$ in the wavelength range $250-1000 \mathrm{~nm}$, and the two RI values for the $\mathrm{Hb}$ and the plasma found in the literature. .......................................... 77

Figure 6-1: A schematic drawing of the experimental setup used in this study. ....................... 82 Figure 6-2: Quantitative phase imaging based on the Mach-Zehnder Interferometer. Phase delay occured due to waves passing through the object under investigation. 83 Figure 6-3: a- Phase shift image and b- Separated Fourier spectra signal of one fringe pattern. . 85 Figure 6-4: Interferogram a- in the absence of a blood sample and b- in the presence of the selected RBC. 89

Figure 6-5: Schematic diagram of the incident waves on a single REB cell showing the refractive index measuring points. 90

Figure 6-6: Three-dimensional thickness profile of single RBC,. The phase information can be converted into the refractive index based on previous knowledge of certain properties. 91 Figure 6-7: Microscopic images of a- healthy RBCs, b- Seckle RBCs obtained from the literature and c- the RBC interferogram of a healthy donor obtained in this study. 92 Figure 6-8: The image processing and cell segmentation process for one interferogram at $70 \%$ hemoglobin concentration of a. Original image, b. Adjusted threshold and contrast c. Low-pass "average filtered" image d. High-pass "Canny filtered" image e. Holes filling process f. Complete cell segmentation and cell counting. 93 Figure 6-9: The results of the experiment and the manual cells count at various hemoglobin concentrations. 


\section{List of Symbols and Abbreviations}

\begin{tabular}{|c|c|}
\hline$\eta_{A b s}$ & Absolute refractive index \\
\hline$\eta$ & Refractive index \\
\hline c & Speed of light in vacuum \\
\hline$v_{\text {Med }}$ & Speed of light in the other medium at \\
\hline$\lambda$ & Light wavelength \\
\hline$\vec{A}$ & Light wave amplitude vector \\
\hline$\vec{k}$ & Wave vector \\
\hline$\varphi_{o}$ & Phase constant \\
\hline$\omega$ & Angular frequency \\
\hline$f$ & Linear frequency \\
\hline$\varepsilon_{o}$ & Vacuum permittivity \\
\hline$\theta_{\text {critical }}$ & Critical angle of the incident light \\
\hline$\phi$ & Phase \\
\hline$u_{i}$ & Standard uncertainty \\
\hline$D_{T}$ & Thermal diffusion coefficient $\left(\mathrm{m}^{2} \cdot \mathrm{s}^{-1} \cdot \mathrm{K}^{-1}\right)$ \\
\hline$S_{T}$ & Soret coefficient $=\left(\mathrm{K}^{-1}\right)$ \\
\hline$T$ & Temperature $(\mathrm{K})$ \\
\hline$\frac{\partial n}{\partial c}$ & Concentration coefficient of refractive index \\
\hline$\frac{\partial n}{\partial T}$ & Temperature coefficient of refractive index \\
\hline$\left(n_{T H N}\right)_{n C_{12}} \mid I B B$ & Refractive index of THN with respect to $n \mathrm{C}_{12}$ at constant IBB \\
\hline$\left(n_{n C_{12}}\right)_{I B B} \mid T H N$ & Refractive index of $n \mathrm{C}_{12}$ with respect to IBB at constant THN \\
\hline$\left(n_{T H N}\right)_{I B B} \mid n C_{12}$ & Refractive index of THN with respect to IBB at constant $n \mathrm{C}_{12}$ \\
\hline $\mathrm{Hb}$ & $\begin{array}{l}\text { Hemoglobin } \\
\text { Micro Mach-Zehnder interferometer }\end{array}$ \\
\hline MMZI & Micro Mach-Zehnder interferometer \\
\hline
\end{tabular}




\section{CHAPTER 1 RESEARCH MOTIVATION AND LITERATURE REVIEW}

\subsection{Introduction}

The refractive index, presumably discovered by Thomas Young in the early eighteenth century, is one of the most important optical properties of transparent and semi-transparent materials. This parameter is defined as the ratio of the velocity of light in air (reference medium) to the velocity of light in the desired material. The refractive index of a material reveals the light interaction behaviour of the material itself.

The refractive index correlates highly with the material density, chemical composition and concentration, temperature, pressure, and the wavelength of the electromagnetic waves illuminating the material [1-9]. Although refractive index changes in individual materials or between different mediums are very small, these changes have a significant impact on the behaviours or characteristics of the material. Precise measurement of the refractive index of materials is therefore of great interest in many areas of engineering and science, including the design and development of optical measurement instruments, the description of optical behaviours in physics, and determinations of material purity and composition in chemistry [1011]. While the refractive index data of most transparent and semi-transparent materials is recorded in physics handbooks, they are not presented for all wavelengths of the electromagnetic spectrum or for different temperatures. The refractive index data presented also varies greatly across publications.

Interference phenomena discovered by Thomas Young in early eighteen century, occurs when two waves generated from the same coherence source superposition or overlapped with one another. Since the discovery of interference phenomena, various interferometric techniques have been developed by engineers and researchers. These techniques include the Fizeau, the Michelson, the Mach-Zehnder, the Sagnac and the Fabry-Perot interferometers. Each of these techniques is classified based on the number of light beams passing through the object under investigation.

Numerous optical measurement techniques have been developed for refractive index measurements. Some of these techniques include optical digital interferometry (ODI) [12-16], refractometry [15,17,18], optical beam deflection (OBD) [19-21], and other techniques [22-25]. 
These techniques are commercially available and have been documented in the literature. One of the main advantages of optical measurement techniques is their ability to provide accuracy relatively close to that of the illumination source wavelengths without any physical interaction with the test materials.

The Mach-Zehnder interferometer was used in the present study as the objective beam passes through the sample only once. This decreases the aberration of light passing through the sample, leading to decreased measurement error and systematic error in the optical path difference of the two beams. Therefore, Mach-Zehnder interferometer is considered the best suited arrangement for providing clear contrast images and measurement of the optical properties of semi-transparent materials, (e.g. biological cells), those producing relatively high light scattering.

Light source is another important factor in interferometry. The light source needs to be carefully selected in order to obtain high quality images and accurate optical measurements. Multi-wavelength white light is proposed in the literature and generally designed based on the Michelson interferometric arrangement. Fringe patterns with chromatic aberrations can be observed with the Michelson interferometer using a white light source. This occurs when the optical path difference of the interfering beams is nearly zero [26]. The disadvantage of using the white-light interferometer associated with the challenge in the signal processing due to the signature influence by different curve distortions on the signal and the limitation measurements range which depends on the spectral width of the white-light source used [27].

Xinhong et al., in their study, used a xenon white light source in a Michelson interferometer and obtained quantitative phase imaging of biological samples [28], the obtained experimental images suffered from signal noise. A modified Mach-Zehnder interferometric arrangement, using white light, was proposed by Liang et.al. [29], reported the results of the measurements of various optical components in the 555-630-nm spectral region and proposed a scheme for the processing of the experimental data in transparent solid materials. The obtained images in their study showed non uniform spacing between the fringes that is only suitable for studying a homogeneous medium.

Despite the fringe image quality and measurement accuracy obtained by the white light interferometers, coherence light sources generated by laser sources with relatively low frequencies and long wavelengths e (compered to blue light), such as the red light generated by a 
stabilized He-Ne laser, provide sharper fringe pattern images and high optical measurement accuracy in biological samples that usually produce high aberration that lead to a reduction image resolution and contrast. These aberrations may induce from imperfections in the optical system the complexity and the physical properties of the specimen which can be overcome using coherence high frequency light source.

In the first part of this study, a Mach-Zehnder interferometer was used to obtain quantitative measurements of the refractive index of ternary liquid mixtures using a coherent light source. The three hydrocarbon components of 1,2,3,4-Tetrtahydronaphtalenene (THN), Isobutylbenzen (IBB), and Dodecane $\left(n \mathrm{C}_{12}\right)$ were chosen because they are simplified multicomponent substances of hydrocarbon in natural crude oil reservoirs [30] and are of great interest in the Differential Scanning Calorimetry (DSC) and Influence of Vibrations on diffusion in Liquid (IVIDIL) experiments currently taking place (on ground and in space) at the International Space Station (ISS) [31].

The optical properties of the pure studied hydrocarbon substances and some of their binary and ternary mixtures can be found in the literature. Mialdun et al., [32], in their study, obtained experimental data for a few ternary compositions using four different instrumental techniques: thermos-gravitational column (TGC) in combination with sliding symmetric tubes (SST), optical beam deflection (OBD), and optical digital interferometry (ODI). Mialdun et al., [33] also studied these mixtures using the Mach-Zehnder interferometer instrument SODI (Selected Optical Diagnostic Instrument) on the ISS of series of the DCMIX (Diffusion Coefficients in Mixtures). Shevtsova et al. [34], in their research, determined the contrast factors from the refractive index values of the same components using the Abbemat refractometer. Platten et al.[35], studied a few binary compositions of the same mixtures using multiple techniques including transient holographic gratings, annular and parallelepipedic thermos-gravitational columns, and vertical parallelepipedic columns with velocity amplitude determination by laser doppler velocimetry. Although all of these studies provided the refractive index of particular ternary mixtures, many compositions are still missing from the literature.

The Abbemat refractometer is an automated compact instrument can be used to measure the refractive index of transparent samples with five digit accuracy. The Abbemat refractometer was used in this study in order to verify the results obtained with the Mach-Zehnder interferometer. It 
was also used to obtain the refractive index of the samples under investigation at multiple wavelengths $[1,36,37]$.

The attempt in the second part of this study was to develop an interferometric system based on the Mach-Zehnder interferometer arrangement that is capable of providing high quality images of the Erythrocytes (red blood cells, RBCs) morphology and provide quantitative measurements of their refractive index.

Blood is one of the most important connective tissues within the human body. Its analysis is the most frequently performed procedure in medical diagnostics because it provides a wealth of information regarding the patients' condition. The primary role of blood is to transport various agents such as oxygen, carbon dioxide, nutrients, wastes and hormones. Blood is a heterogeneous medium consisting of cells suspended in plasma. Blood plasma is composed of approximately $90 \%$ water and $10 \%$ protein. RBCs represent almost $99 \%$ of all blood cells. The remaining $1 \%$ is made up of leukocytes (white blood cells, WBCs) and thrombocytes (platelets), as shown in Figure 1-1.

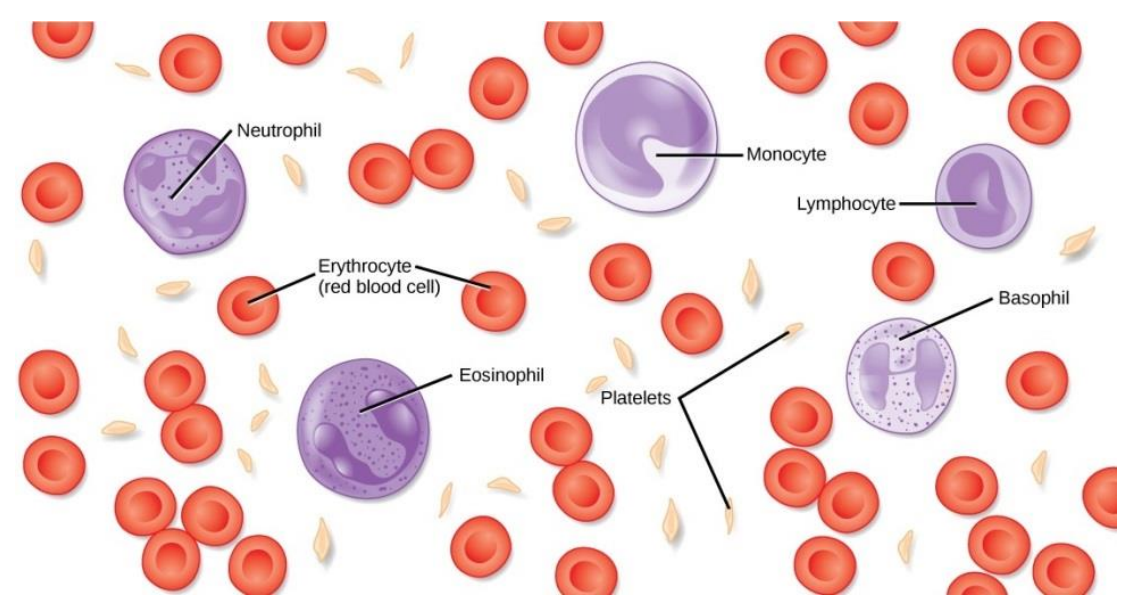

Figure 1-1: Whole human blood showing the RBCs, platelets and the different white blood cells. http://voer.edu.vn/m/components-of-the-blood/c74c1590.

RBCs are non-nucleated, round, biconcave, disc-shaped cells with a thick rim and a thin sunken centre (minimum thickness $T_{\min }=0.81 \pm 0.35 \mu \mathrm{m}$ and maximum thickness $T_{\max }=$ $2.58 \pm 0.27 \mu \mathrm{m})$ with typical geometrical dimensions of $6.2-8.2 \mu[38,39]$. The two primary functions of RBCs are to deliver oxygen to the cells of the whole body and to remove waste and carbon dioxide. The leukocytes or white blood cells are the biggest cells in the blood and are involved in the body's immune system which attacks and remove viruses and bacteria from the 
body. Typical levels of leukocytes in humans range between 4,000 and 10,800 cells per microliter of blood. Low WBC counts may indicate a risk of infection while high WBC counts may indicate an existing infection, tissue damage or leukaemia. The platelets, which are very small, form the clots that prevent bleeding after injury.

Blood screening is a medical process used to test for particular diseases or conditions such as pregnancy, general infections and cancer. Researchers have designed and developed numerous medical devices and techniques to obtain the desired properties of blood. The flow Cytometry (CFC) is one of the most current conventional clinical diagnostic techniques (also known as hematology analysers). This is a powerful technique which analyses multiple parameters within the blood statically and provides a cellular characteristic report (size, complexity and health) [40]. In this technique, cells are passed through an aperture containing an electrical current or through a laser beam, capturing the light that emerges from each cell as it passes through.

Impedance-based hematologic analyzers allow blood cells to pass through individual electrical currents. As such, the cells add resistance causing an amplitude change in the resistance measurement or the pulse. Each pulse is then counted as one blood cell. The amplitude of the pulse is directly related to the size of the cell. Smaller cells cause less resistance lower amplitude.

Laser-based hematologic analyzers on the other hand, allow blood cells to pass through a flow cell containing a laser in single file. As the blood cells pass through the laser beam, part of the beam passes through the cells toward a forward detector. This provides information regarding the number of cells. The other part of the beam is deflected off the cells and directed toward the scatter detector. This provides information regarding the volume and the complexity of the cells.

Both of these methods provides limited statistical information about the blood cells and rely on the assumption that only a single cell passes through the testing beam at one time. Since blood contains cells of various sizes, it is possible that more than one cell passes through the testing beam at one time, leading to significant error that cannot be ignored. The other drawback of these systems is that they only provide limited information about the blood sample such as the size and number of cells. In addition, these methods use a large amount of blood and require professional labour and effort.

Advanced digital microscopies are other critical diagnostic tools. The output images from these instruments are used to provide an estimate of the complete blood count (CBC) and aid in 
the identification of blood cell malformations, disorders, infections, allergies and clotting problems.

The panel of tests used to examine different blood particles includes the WBC and RBC counts, WBC differential, signs of disease and the number of infected cells [41]. In these methods, the blood film should be stained (e.g. May-Grunwald-Giemsa stain (MGG)) and then viewed with a transmission light microscope. The MGG staining method involves many stages [41,42], making it time and labour intensive. This method also requires a customized infrastructure (i.e., specialized instruments, dyes and trained personnel). This is a time consuming task that is susceptible to human error.

The optical behaviour of blood is primarily determined by the optical properties of RBCs. The microphysical properties of blood (size distribution, functions of formative blood elements, specific concentrations, scattering, absorption and refractive indices) allow for the evaluation of the medical state of the human organism and for the diagnosis of multiple diseases. Many diseases have a significant impact on the shape and optical properties of RBCs. The refractive index of human blood is considered to be the most important optical property and provides valuable information regarding the specimen under investigation.

Measuring the refractive index of biological cells is important in order to obtain vital information such as haemoglobin concentration, average cell mass and cell thickness. Very few measurement techniques have received wide acceptance in clinical practice due to exceptionally complicated experimental procedures or limitations in the methodological principles, limiting the accuracy of diagnoses [43]. Furthermore, the majority of the techniques in existence require that measurements be made in isolated erythrocyte samples since they depend on attenuation or absorption coefficients that are contingent upon the absorption of transmitted radiation by the RBCs as well as the plasma and other biological components of the blood.

There has been recent progress in the development of advanced phase microscopic techniques that provide quantitative information about refractive index variations in cells and tissues. These methods include Fourier phase microscopy [44], digital holographic microscopy [45-49], Hilbert phase microscopy [50,51], diffraction phase microscopy [52], tomographic phase microscopy [53], and the spatial phase-shifting method [54]. Unfortunately, these methods are expensive and provide specific information about RBCs. Recording non-invasive, high resolution 3D images of living cells in real time is technically challenging. The majority of 
biological cells are transparent and differ very little from their surroundings in terms of optical properties such as absorbance and reflectance.

The single cells of many biomedical tissues, including the blood cells, are transparent to incident light. Changes in the optical path length (OPL) occur as a result of variations in the thickness of the object, causing variation in the refractive index. A phase shift or phase deformation is generated when the sample under investigation is placed in the path of the objective beam in the interferometric system. The quantitative optical parameters of the object causing the phase shift can be obtained using phase shift analysis.

This study attempted to provide highly accurate experimental measurements of the refractive indices of transparent and semi-transparent liquid mixtures consisting of the hydrocarbon substances that are missing in the literature and demonstrate quantitative phase imaging and refractive index measurement of human RBCs using phase-shifting interference. The system was designed based on the Mach-Zehnder optical arrangement and proved successfully of providing non-invasive quantitative phase images and optical properties of RBCs with nanoscale sensitivity using the Fourier transform method.

The results of this study could provide opportunity to the researchers to elaborate on noninvasive clinical diagnostic methods with minimal operational time, effort and expertise, allowing for immediate clinical decisions.

\subsection{Research Objectives}

In the past few decades, there have been significant calamities that have impacted the environment, especially in developing countries, and these changes have affected their ecology. The cardiovascular system is one of the most sensitive systems within the human body. Research has shown a significant increase in blood cancers in humans. In fact, these cancers are the second leading cause of death in developing countries. The survival rates tend to be lower in these countries and this is most likely due to a combination of late stage diagnosis and a lack of access to standard treatment $[55,56]$. Furthermore, specialists tend to be scarce in these countries. An economical, compact and semi-automated medical diagnostic system for biological tissues would increase early detection and treatment, increasing cancer survival rates and public health. A comprehensive literature search has revealed that such systems are unavailable or very limited.

There is a twofold purpose to this research study. The first goal was to design and develop an interferometry system that is capable of providing precise measurements of the refractive 
indices of transparent and semi-transparent mediums. The second goal was to modify this system to create a simple medical diagnostic micro-interferometric system that can be operated with minimal effort and expertise. The result could then be shared with medical professionals and experts for medical advice.

During the development phase, the system was used to take precise measurements of the temperature and concentration derivatives of the refractive index of ternary hydrocarbon mixtures. The mixtures were selected due to their limited coverage in the literature and their importance to the Differential Scanning Calorimetry (DSC) and Influence of Vibrations on diffusion in Liquid (IVIDIL) experiments currently taking place (on ground and in space) at the International Space Station (ISS) [31], and Canadian Space Agency. This interferometric system was then modified into Micro Mach-Zehnder Interferometry (MMZI) and used to take images and measure the refractive index and morphological structure of living biological cells. There has been more emphasis places on this technique in the field of biological studies.

The first part of this study includes the design and development of the interferometric system using Mach-Zehnder Interferometry (MZI). The following points summarise the main achievements of this stage:

- The design and construction of an experimental interferometer setup based on a Mach-Zehnder arrangement that is capable of providing precise measurements of the refractive index of various transparent and semi-transparent mediums.

- A new development of the MZI cell holder prominently featured in the literature that achieved precise measurements of contrast factors for ternary mixtures.

- Precise experimental measurements of the refractive indices and temperature coefficient contrast factors for the entire concentration range of the three hydrocarbon components 1,2,3,4-Tetrtahydronaphtalenene (THN), Isobutylbenzen (IBB), and Dodecane $\left(n \mathrm{C}_{12}\right)$ at different ternary compositions.

- Developed and validated empirical correlations which can be used to predict the refractive index of ternary mixtures as a function of concentration, temperature and wavelength with an average relative accuracy of $1.55 \times 10^{-3}$. This level of accuracy is comparable to that of the best mixing rules by order of magnitude.

- Precise measurements of the temperature and concentration derivatives of the refractive index of two ternary systems formed by three hydrocarbon components, 


1,2,3,4-Tetrahydronaphtalene (THN), Isobutylbenzene (IBB), and Dodecane
$\left(n C_{12}\right)$ For each system we considered three different contrast
factors: $\left(n_{T H N}\right)_{n C_{12}}\left|I B B,\left(n_{C_{12}}\right)_{I B B}\right| T H N$ and $\left(n_{T H N}\right)_{I B B} \mid n C_{12}$.mixtures.

Optical interferometry is a non-invasive method that can be used to obtain images and measure the optical properties of living cells with nanoscale accuracy. The second part of the study involved the modification of MZI into MMZI. At this stage the system was be able to provide quantitative phase measurements of biological tissues. The following points summarise the main achievements of this stage:

- Measured temperature and concentration dependent refractive indices of human blood for a wide range of visible coherence light.

- Developed and validated empirical model which can be used to predict the refractive index at any temperature and haemoglobin concentration.

- Quantitative 3D imaging: optical phase delay information can be quantitatively related to the physical and chemical properties of the sample.

- Non-invasive and real time imaging of living cells.

- Wrote custom software in the Matlab® environment in order to analyse video sequences of RBCs. The software converts the video into frames and then calculates the number and surface area of RBCs for each frame to estimate the hemoglobin concentration.

Being able to take images of cells and measure the refractive index has opened the door for direct analysis of live cells and their pathophysiological alterations. These results can also serve different biomedical purposes. For example, variations in the refractive index of cells can serve as an indication of infection by foreign micro-organisms. Research has found that RBCs have different refractive indices at different growth stages of a plasmodium falciparum infection, a malaria causing parasite [41]. Accurate measurement of the refractive index of RBCs could then help in the diagnosis of plasmodium falciparum infections and in tracking the progress of the disease. 


\section{CHAPTER 2 INTRODUCTION AND BACKGROUND}

Optical interferometry is a non-contact method used for the imaging and measurement of the optical properties of small or large objects with nanoscale accuracy. Interferometers are essential optical systems in medical science and are generally used to measure refractive index changes, cell pathology and shape irregularities. The ability to provide quantitative, non-invasive measurements makes interferometers suitable candidates for the study of living cells in vivo and in vitro applications.

The refractive index is one of the most important parameters in scientific research areas such as physics, chemistry, biomedical sciences and industrial application [57,58]. There are strict requirements for the precision and accuracy of optical components and optical systems. The measurement precision of refractive indices is of great interest in various fields and is rapidly increasing with the development of new technologies. In some cases, the refractive index data cannot be found in reference books. Furthermore, the data vary in the literature. Small differences in the refractive index of various solutions can be very significant. This project was motivated by the many possibilities for easy, accurate and cost effective optical properties measurement. Quantitative measurements of the optical properties of transparent and semitransparent mediums can be obtained using the coherent light source interference phenomenon.

In this study, the optical refractometer and interferometer techniques were used to obtain precise measurements of the refractive indices of transparent mediums. The working principle of the refractometer is based on the measurement of the total refraction angle of the incident wave at the interference between two mediums. Interferometry, on the other hand, is the science of combining light waves in order to cause interference. Various interferometry techniques have been developed since its discovery. These techniques have been widely used in science and industry for the measurement of small displacements, refractive index changes and surface irregularities. In addition, interferometry has the precision and potential accuracy required for most optical tests. Since all of the experimental measurements used in this study depend on the use of light properties and the interference phenomenon, it would be appropriate to begin with the basic working principles of the interference phenomenon as well as its application as a method of measurement in scientific research. This requires basic knowledge of the properties of light waves as they are the primary cause of this phenomenon. 


\subsection{Light Waves}

Many phenomena in our everyday lives depend on the properties of light. When light propagates inside a material, it no longer travels at the same speed as a light wave in a vacuum; Light wave speed is slower when travelling through any other medium or material.

The absolute refractive index $\eta_{A b s}$ of a material is a unitless ratio of the speed of light in vacuum $\mathrm{c}$ to the speed of light in the other medium $v_{\text {Med }}$ at wavelength $\lambda$. The formula is as follows:

$$
\eta_{A b s}=\frac{c}{v_{M e d}}
$$

The index of refraction, or refractive index, is a basic optical property of any material. The refractive index of a vacuum is known to be exactly 1.00. However, in practice, refractive index $\eta$ refers to the index relative to $\eta_{\text {Air }}=1.00$ [59]. The absolute refractive index of air is 1.00029, so the difference is small but not negligible. Unless otherwise stated, all refractive index values in this dissertation are relative to $\eta_{\text {Air }}=1.00$. In modern physics, light is described as electromagnetic waves and a current of particles called photons. The interference phenomenon of light is understood through the properties of its waves. Electromagnetic waves, including light waves, propagate as a result of mutual oscillation of the electric and magnetic fields perpendicular to the direction of propagation, a characteristic of transverse waves. If the electric and magnetic fields oscillate in a particular direction throughout the propagation of the wave, the light is linearly polarized. When solving the equation for a wave of linearly polarized light in 3D, the real part elementary solution of the electric field is as follows

$$
\vec{E}(x, y, z, t)=\vec{A} \cos (\vec{k} \cdot \vec{r}-\omega t)
$$

where $\vec{A}$ is the amplitude vector, and $\omega$ is the angular frequency which has the following relation to the linear frequency $f$

$$
\omega=2 \pi f
$$

The magnitude of $\vec{k}$ is known as wave number, and can be obtained using

$$
|\vec{k}|=\frac{2 \pi}{\lambda}
$$

where $\lambda$ is the wavelength of the light. The frequency and wavelength are related to each other in the following manner

$$
c=\lambda f
$$


The entire cosine argument is called the phase. $\omega t$ represents the time variation in the phase and $\vec{k} \cdot \vec{r}$ represents the spatial variation in the phase. The human eye (and other devices) is only sensitive to the intensity of light. The intensity of a plane wave light in a vacuum can be calculated as follows

$$
I=\varepsilon_{o} c\left\langle E^{2}\right\rangle=\frac{1}{2} \varepsilon_{0} c A^{2}
$$

where $\varepsilon_{o}$ is vacuum permittivity and the angular bracket indicates a time average, the wave equation is a complex equation since its contained a combination of amplitude and phase information, therefore the wave equation Eq. 2.2 can be written in the complex form

$$
\vec{E}(x, y, z, t)=\vec{A} e^{i(\vec{k} \cdot \vec{r}-\omega t)}
$$

The phase information is stored in the interferogram of the interference pattern. The concept of interference will describe in section 2.2 .

\subsection{Refractometer}

The speed of light travelling through a medium is different than its speed when travelling through the air or in a vacuum due to the mismatched refractive indices of the two mediums. Changes in the speed of light cause changes in the wave's direction (deflection). The change in the wave's direction depends on the difference between the refractive index of the two mediums as well as the angle of incidence.

The working principle of the refractometer technique is based on determining the critical angle of the total reflection, where the incident light waves are completely reflected with a $90^{\circ}$ degree angle to the normal position. The incident light waves with angles greater than the critical angle will only experience reflection at the interface surface and no refraction will be observed (see Figure 2-1).

The ratio of the sins of the two angles is equal to the opposite ratio of the refractive indices of the two media. This relationship is called Snell's law [88] and given by the following expression

$$
\frac{\sin \theta_{1}}{\sin \theta_{2}}=\frac{v_{1}}{v_{2}}=\frac{\eta_{2}}{\eta_{1}}
$$

Based on Snell's law, the critical incident angle $\theta_{1}$ that is led to a total refraction angle $\theta_{2}$, since $\sin \theta_{2}=\sin 90=1$, and the refractive index of the air $\eta_{1}=\eta_{\text {air }}=1$, thus Eq. 2.8 can be simplified to 


$$
\eta_{2}=\sin \theta_{\text {critical }}
$$

Hence, the refractive index of unknown medium $\eta_{2}$ can be ascertained if the critical angle $\theta_{\text {critical }}$ of the total internal reflection is known. This phenomenon is referred to as total internal reflection.
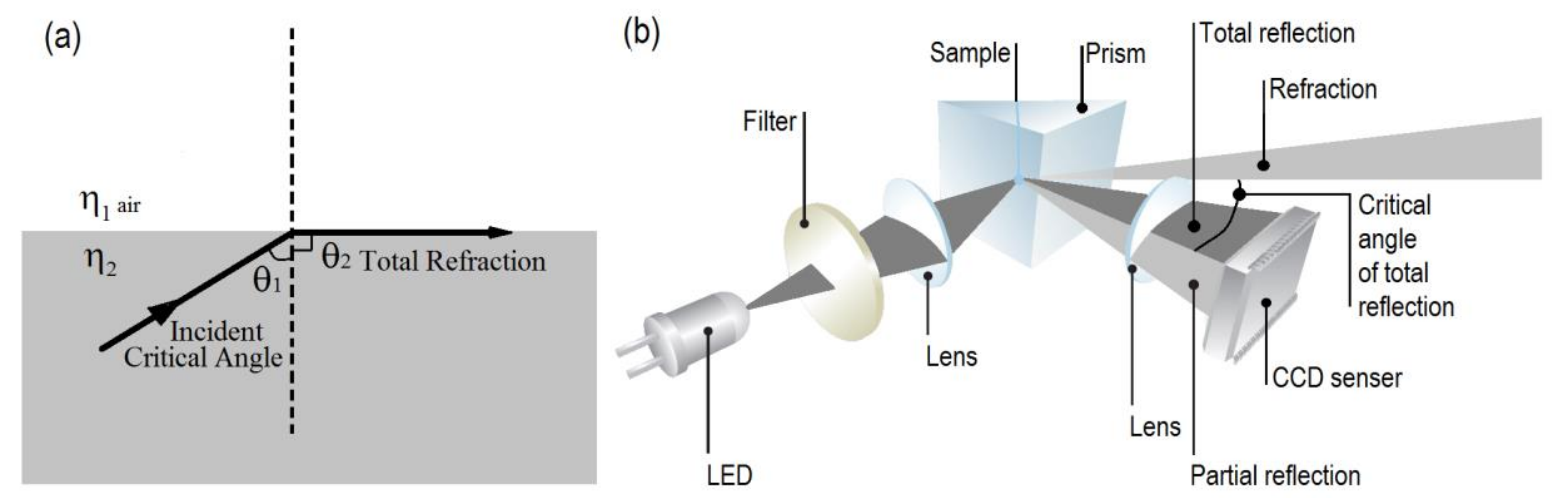

Figure 2-1: Schematic diagram of a Refractometer illustrating, a-The Incident light waves and the critical angle and $b$ - The working principle of the refractometer.

http://www.anton-paar.com/ca-en/products/group/refractometer/\#RefractiveIndex

\subsection{Interferometry}

Interferometry is the science of combining light waves to cause interference. As two beams of light occupy the same space at the same time they tend to overlap (interfere) with one another at some point, causing areas of constructive and destructive interference. This interference manifests as a series of light and dark bands in 2-D space, known as fringes. If two waves emitted from the same coherence source given by

$$
\begin{aligned}
\vec{\psi}_{1}(r, t) & =\vec{A} e^{i(\vec{k} \cdot \vec{r}-\omega t)} \\
\vec{\psi}_{2}(r, t) & =\vec{A} e^{i(\vec{k} \cdot \vec{r}+\vec{k} \cdot \delta-\omega t)}
\end{aligned}
$$

The interference occurred by the two waves overlapping on each other in the space $\vec{\psi}=\vec{\psi}_{1}+\vec{\psi}_{2}$ and the total interference magnitude can be given by

$$
\vec{\psi}(r, t)=2 \vec{A} \cos (\vec{k} \cdot \delta / 2) e^{i(\vec{k} \cdot \vec{r}-\omega t+\vec{k} \cdot \delta / 2)}
$$

where $2 \vec{A} \cos (\vec{k} \cdot \delta / 2)$ is the new amplitude of the signal and $e^{i(\vec{k} \cdot \vec{r}-\omega t+\vec{k} \cdot \delta / 2)}$ is the time depended phase shift. When the waves intersected in phase, constructive interference and a bright area is display (light intensity maxima). This phenomenon occurs at $\delta=\mp n \lambda$, where $\delta$ represents the path difference between the two waves, $\lambda$ represents the wavelength of the electromagnetic wave and $(n=0,1,2, \ldots)$ integer number. If $\delta=\mp(n+1 / 2) \lambda$, the two waves 
intersect out of phase and subtract from one another, destructive interference and a dark area is display (low or zero light intensity, minima). Both constructive and destructive interferences are based on the superposition principle which states that the amplitude of the overlapping waves is added algebraically, as illustrated in Figure 2-2. The number of maxima and the minima can be obtained using $2 d / \lambda$, where $\mathrm{d}$ is the separation distance between the two light sources. These phase changes correspond with the difference between the path lengths of the interfering waves of light.

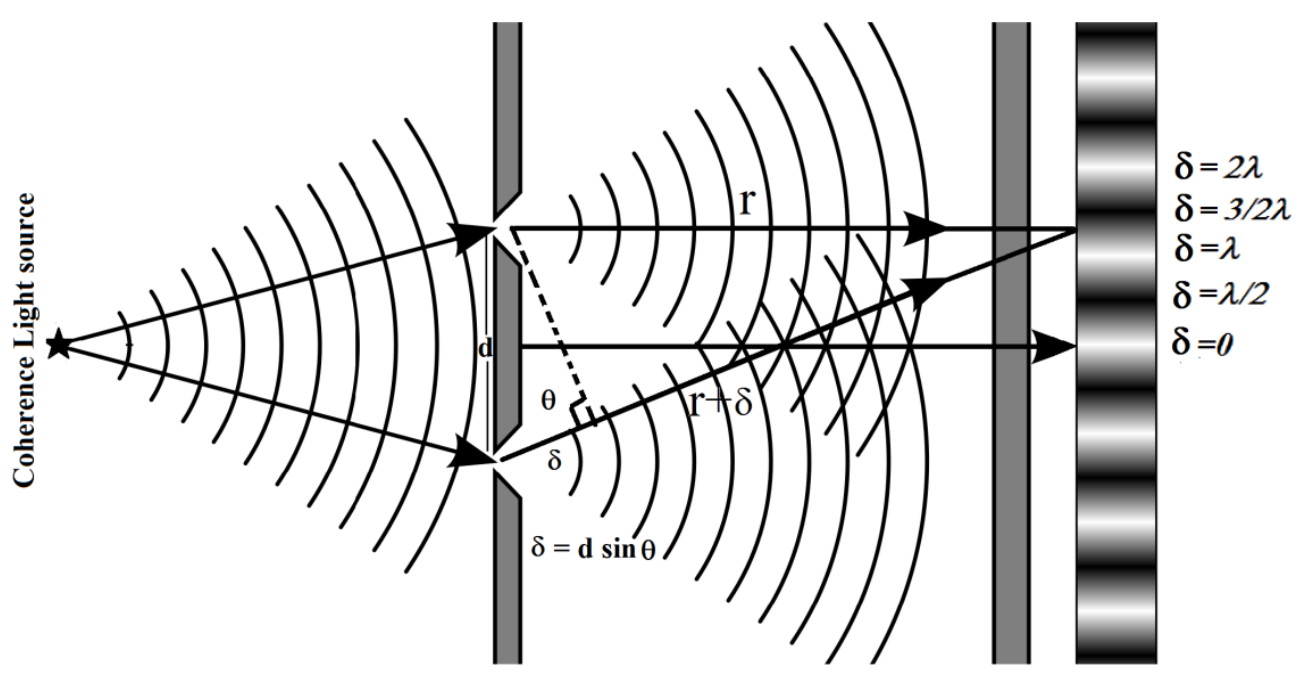

Figure 2-2: Interference can be formed by the superposition (addition) of two traveling waves, of equal amplitude and frequency forming, fringe pattern known as interferogram.

The movement of the interference bands, referred to as a fringe shift, can be used to quantify the parameters that modify the path length of the light waves. These parameters include the refractive index $(\eta)$, the geometry of the system, or any other property that is a function of these parameters such as temperature, pressure, and concentration. Physically, the thickness of each fringe at any point represents a path length equal to the half wavelength of the light used to create the fringes. Common practice is therefore to measure the fringe shift based on the number of fringes to pass a certain point on the observation screen. This number is directly related to changes in the physical path of the light which can be used to quantify a multitude of physical phenomena.

In addition, modern technology e.g. computers and software, has transformed interferometry into a very powerful technique used in many other fields, including astronomical imaging, microfluidics, surface flatness testing and mechanical stress/strain measurement [60-65]. 


\subsection{Basic Types of Interferometers}

There are two main types of interferometers: two beam interferometers and multiple beam interferometers. A typical interferometer involves an optical arrangement in which a beam splitter splits a light beam generated from one coherent light source into two identical beams (a reference beam and a measurement or objective beam). A beam splitter is a partially reflecting metal or dielectric film on a transparent substance. Polarizing prisms and diffraction gratings can also be used to split light beams. These beams are derived from the same source but travel along separate paths. The measurement beam is either reflected or passes through the medium under investigation. These experiments can be set up in many different configurations depending on their application.

There are several common types of interferometers used in both research and industry. The most common two-beam interferometers are the Fizeau, the Michelson, the Mach-Zehnder and the Sagnac interferometers. The most common multiple-beam interferometer is the Fabry-Perot interferometer. These interferometers are discussed in more detail in the following section.

\subsubsection{The Fizeau Interferometer}

The Fizeau interferometer, illustrated in Figure 2-3, is an interferometric arrangement where interference fringes of equal thickness are formed between two flat surfaces separated by an air gap and illuminated with a collimated beam.

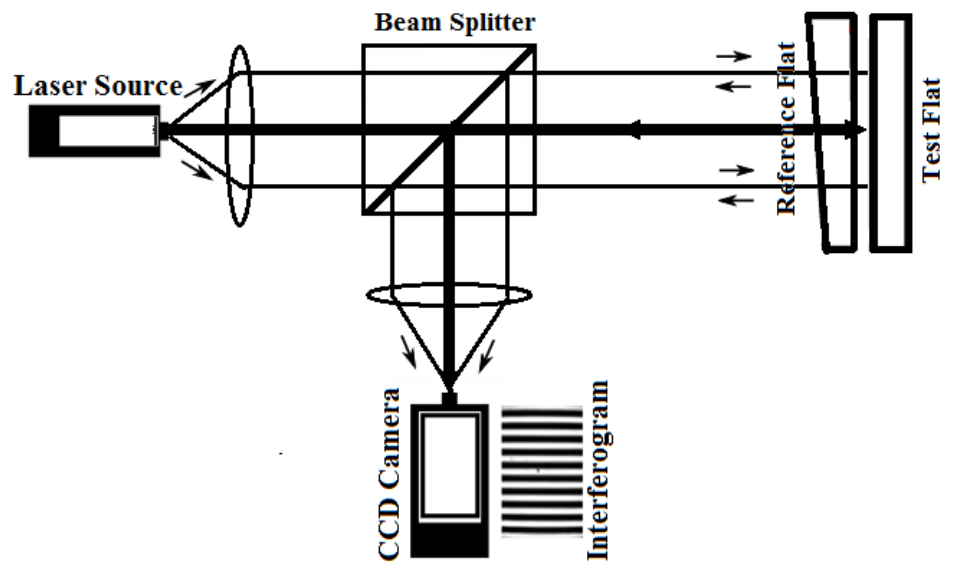

Figure 2-3: A Fizeau interferometer arrangement whereby two reflecting surfaces are placed facing one another.

If one of the surfaces is a standard reference flat surface, the fringe pattern serves as a contour map of the errors in the test surface. Modified forms of the Fizeau interferometer are used to test convex and concave surfaces by using a converging or diverging beam. 


\subsubsection{The Michelson Interferometer}

The Michelson interferometer, shown in Figure 2-4, is the oldest and simplest type of interferometer. This interferometer is used for many scientific applications including measurements of highly sensitive deflection, flatness, temperature and refractive index [66]. The Michelson interferometer uses a coherent light source (such as a laser) which emits a single beam that is then divided by a beam splitter into two identical beams. These beams are then reflected by mirrors back towards the beam splitter. During this event, one beam is passed through a glass plate in order to compensate for the phase difference caused by the thickness of the beam splitter.

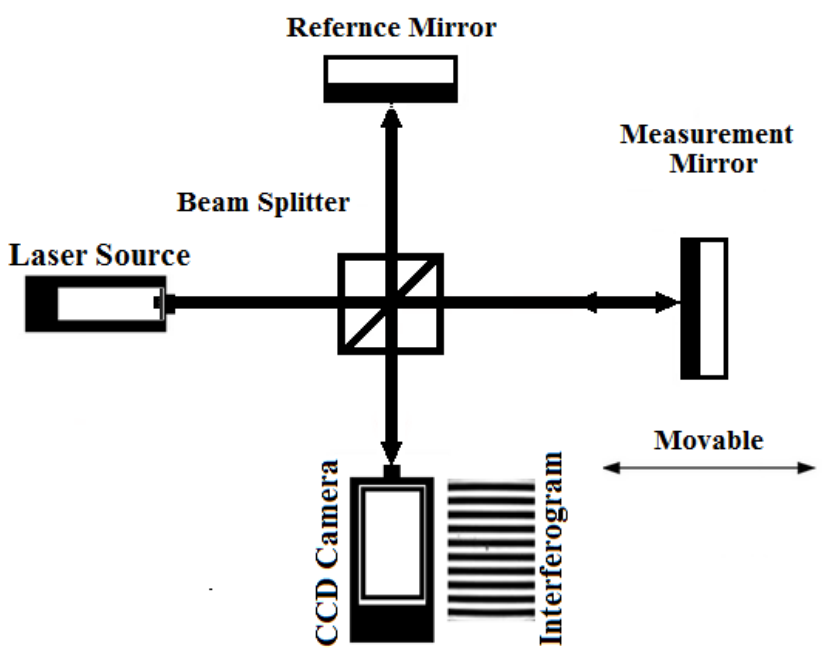

Figure 2-4: Michelson interferometer arrangement.

When the apparatus is properly aligned, the light waves recombined causing interference fringes which can be viewed on an observation screen, a photo detector or CCD camera. This configuration is easily implemented as a deflection measurement device since a mirror can be attached to a system of interest with ease. As the mirror moves there is a path length difference in one arm of the interferometer causing a fringe shift. Deflection measurements have been reported as low as $0.1 \mu \mathrm{m}$ [67], depending on fringe shift detection capability. The TwymanGreen interferometer is a Michelson interferometer that has been modified to use collimated light. This interferometer is extensively used in optical testing.

\subsubsection{The Sagnac Interferometer}

This is another common type of interferometer. In this arrangement, shown in Figure 2-5, three mirrors are used in conjunction with a beam splitter to send the divided rays of light in 
opposing circular paths. This arrangement is especially useful for the measurement of angular velocity and acceleration. As the device is rotated in a particular direction, the velocity of the light in that direction is decreased relative to the light travelling in the opposite direction. This velocity difference manifests itself as a phase shift, causing a measurable fringe shift which can be related back to the angular velocity of the device.

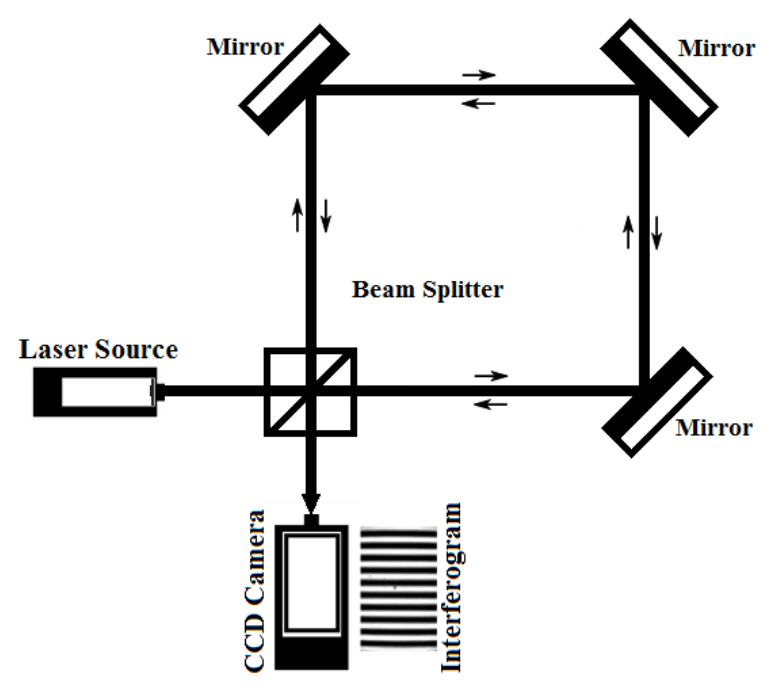

Figure 2-5: Sagnac Interferometer arrangement.

One limitation of this arrangement is that the device must be rotated to produce a response. It is thus desirable to make it as small as possible in order to minimize its inertial effects on the system of interest.

\subsubsection{The Mach-Zehnder Interferometer}

The Mach-Zehnder interferometer is frequently used to perform temperature and refractive index measurements. In this arrangement, an incoming beam is divided by a beam splitter. The separate beams are then reflected from mirrors and directed towards a second beam splitter which recombines them, causing interference fringes. This arrangement is especially useful for small transparent samples which can be placed into one arm of the interferometer, as shown in Figure 2-6. The insertion of such a sample causes a path length change between the two beams, resulting in a fringe shift. This configuration is also useful for the measurement of the quality and flatness of optical components, the thickness of transparent media and the refractive index of optically transparent samples. 
One limitation of this arrangement is that the light only makes a single pass through the sample. Multiple passes through the sample can lead to double the detection sensitivity, as seen with other configurations such as the Michelson interferometer and the Fabry-Perot interferometer.

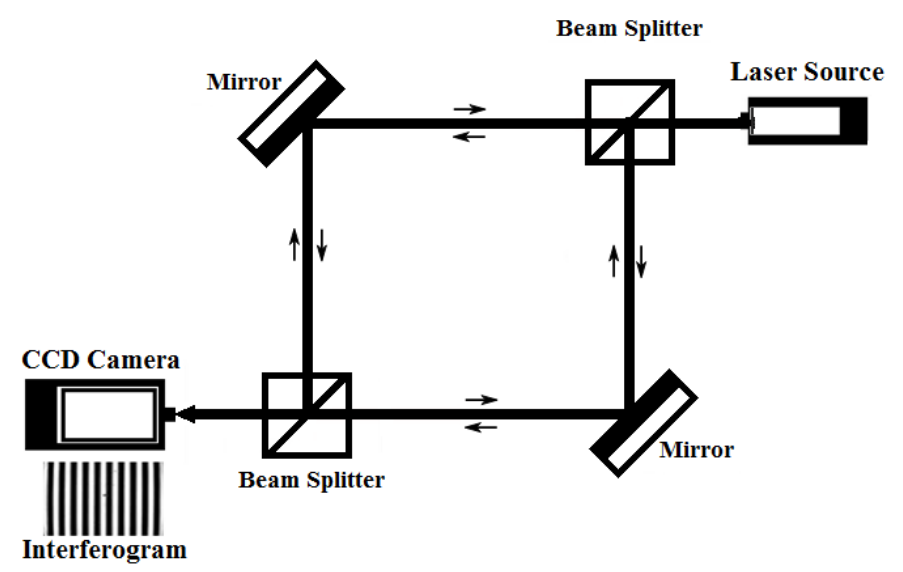

Figure 2-6: Schematic diagram of a Mach-Zehnder interferometer.

\subsubsection{The Fabry-Perot Interferometer}

Another common arrangement is the Fabry-Perot interferometer shown in Figure 2-7. This configuration uses two parallel half silvered plates to reflect a beam several times through a medium. Since the plates are only partially reflective, a portion of the light is transmitted upon each reflection resulting in a group of transmitted rays at one side of the apparatus and a group of reflected rays at the other. The transmitted or reflected rays are then analysed to determine information such as the value of refractive index $(\eta)$, the RI of the medium between the plates or the wavelength of the light of the incoming beam. Since the interference pattern is composed of multiple reflected beams, it is classified as multiple source interference and the fringe pattern obtained is very different than those obtained by the other interferometric arrangements.

The formation of these unique fringes is dependent upon the phase of each exiting beam. A large peak of constructive interference is observed when multiple beams are in phase with one another. When the beams are out of phase we observe an area of destructive interference.

The disparity between these areas and their width is directly related to the construction of the interferometer. This includes the wavelength of the light, plate spacing, RI of the material occupying the separation, plate reflectivity and beam impingement angle [68].

With the exception of the impingement angle or plate separation, these parameters usually remain constant. The device is commonly used to determine the wavelength of the incoming 
beam by modifying one of these variables. Since there is constructive interference between the existing beams, the maximum interference will occur when the beams are in perfect phase [69].

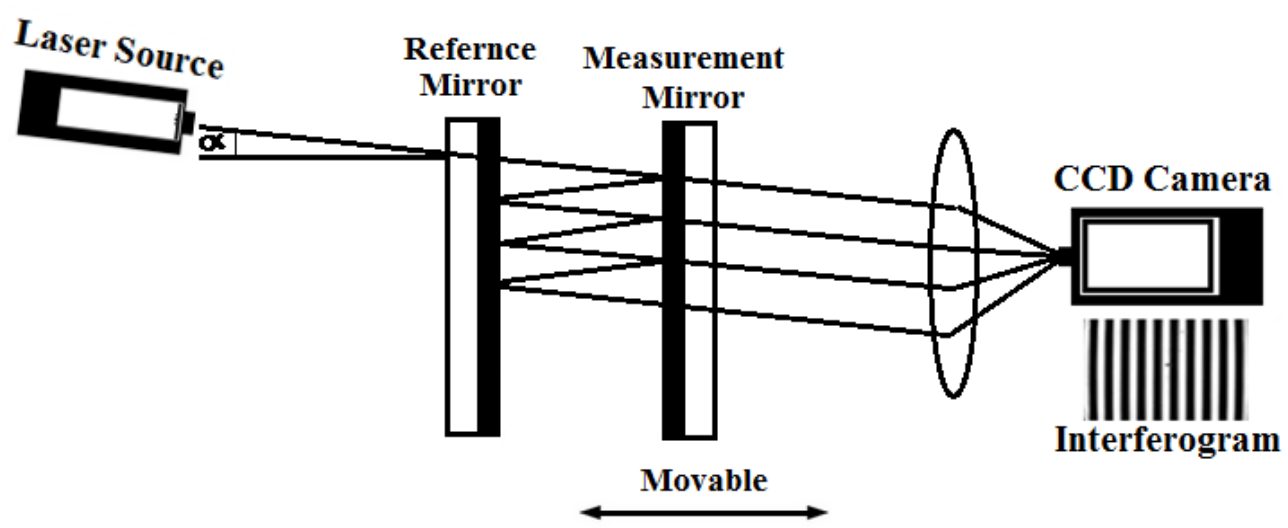

Figure 2-7: Fabry-Perot Interferometer arrangement.

Plate separation variation is done until the maximum intensity of the constructive interference peaks occurs, allowing for the extraction of the wavelength through the known physical relationships of the geometry of the interferometer. In short, by varying plate separation, the Fabry-Perot interferometer is used to determine the wavelengths present in a light source.

\subsection{Interference Principle}

As previously mentioned, interference is the superposition of two or more waves in space. If each wave is described as $\psi(r, t)$, the superposition of these waves can be obtained using $\psi(r, t)=\sum_{i} \psi_{i}(r, t)$. The formulas for the two monochromatic and polarized plan laser source waves, travelling the same distance on different paths, used in this work are

$$
\psi_{1}(r, t)=A \cos (\omega t)=\operatorname{Rel}\left(A e^{i(\omega t)}\right)
$$

and

$$
\psi_{2}(r, t)=A \cos (\omega t+\varphi)
$$

Since these waves are polarized in the same direction, we can treat them as having scalar amplitudes. If $\psi_{1}(r, t)$ and $\psi_{2}(r, t)$ are superimposed, the resultant interference magnitude will be $\psi=\psi_{1}+\psi_{2}$. Then, the intensity $\mathrm{I}=|\psi|^{2}$ term of Eq. 2.13 is obtained using

$$
\begin{aligned}
I & =\left|\psi_{1}+\psi_{2}\right|^{2}=\left(\psi_{1}+\psi_{2}\right)\left(\psi_{1}+\psi_{2}\right)^{*} \\
I & =I_{1}+I_{2}+2 \sqrt{I_{1} I_{2}} \cos \varphi \\
\bar{A} & =2 A \cos (\varphi / 2)
\end{aligned}
$$




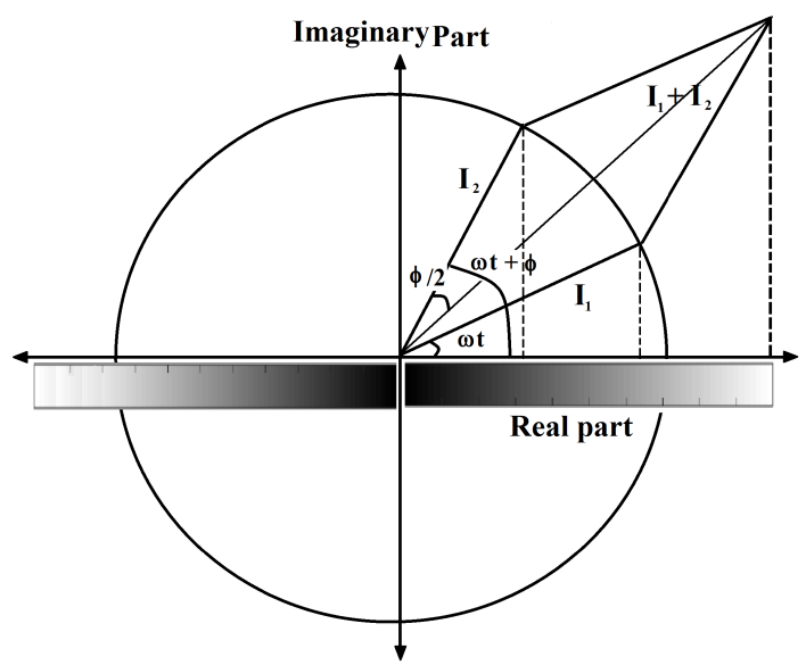

Figure 2-8: Schematic of the amplitude and intensity calculations occur in one interference cycle.

Assuming the individual waves have the same amplitudes and intensities $I_{1}=I_{2}=I$, and $\cos \varphi$ represents the interference phase, see Figure 2-8. From Eq. 2.16 and Eq.2.17, we can see that the intensity of the superposition is the sum of the intensities of the individual waves plus an intensity which is modulated by the cosine of their phase differences, and have new amplitude $\bar{A}$ which equal double of their amplitude multiplied by the cosine half of the phase between them. The above equation is only true if $\varphi$ constant when the beams are travelling in the same medium. These types of beams are called coherent beams. In order to obtain two coherent beams, the phase of the two beams must not drift in time or distance. These can be obtained using laser beams. For this thesis, the coherence factor of interference was achieved by using a single laser source split into two coherent beams by a beam splitter. The distribution of the interference pattern is determined by the relative phase of the two beams.

\subsection{Interference Analysis}

The interferogram images captured by the CCD camera, known as fringe patterns, hold all of the necessary phase information. The fringe analysis is conducted in order to convert the fringe pattern into a distribution of its phase and contrast. This distribution can then be connected to the physical parameters that cause the fringes. There is also a process used to convert wrapped phase maps into unwrapped phase maps, allowing us to obtain the physical parameters required.

The literature suggests two different methods for the analysis of interferogram images in order to determine the phase change from the fringe: Phase Shifting Interferometry (PSI) and Fast Fourier Transform (FFT) [37,70]. Digital Image Subtraction is another less frequently used 
method. These methods are categorized based on the number of input fringe images required for the phase extraction. Both the PSI and FFT methods have proven to be successful at extracting phase information from interference fringe patterns [71,72]. The PSI method requires a minimum of three phase-shifted interferogram to obtain the wrapped phase map [73-75]. The FFT method on the other hand, requires only one phase-shifted interferogram to determine the phase mapping without the need to locate fringe centres or assign fringe orders. In the PSI method, phase shifts are induced by moving a mirror, tilting a glass plate or moving a grating or rotating wave plate.

Using sine function, the intensity distribution of fringe patterns is generally expressed as:

$$
\begin{aligned}
& I(x, y)=I_{o}(x, y)(1+v(x, y) \cos [\phi(x, y)]) \\
& I(x, y)=a(x, y)+b(x, y) \cos \phi(x, y)
\end{aligned}
$$

where $I_{o}(x, y)$ and $v(x, y)$ represent the bias intensity and modulation depth of the fringes in the field of view. This is essentially the background intensity distribution and contrast heterogeneity, and can therefore be substituted with $a(x, y)$ and $b(x, y)$ for simplicity. In most cases, the required value is the phase distribution $\phi(x, y)$. The goal of phase extraction is to find the wrapped phase image in the digital interferogram obtained. In order to better understand the process, let's begin by going over some critical background information.

\subsubsection{Phase Shifting Method}

PSI allows for faster measurements and higher measurement accuracy (1/1000 fringe). The goal of PSI is to insert a time-varying phase shift between the test and reference wavefronts. This is usually done by using a piezoelectric transducer to translate the reference surface, and then decoding the relative phase from the time-varying signal at each measurement element in the interferogram. With the time-varying phase shift $\delta(t)$, the intensity of the interferogram can be expressed as:

$$
I(x, y)=I_{o}(x, y)(1+v(x, y) \cos (\Delta \phi(x, y)+\delta(t)))
$$

In the four step method, four interferograms are recorded and digitized. A fixed $\pi / 2$ phase shift is introduced between adjacent ones; $\delta(\mathrm{t})$ takes on the following four discrete values:

$$
\delta_{i}=0, \pi / 2, \pi, 3 \pi / 2 \quad i=1,2,3,4
$$

which gives the following intensity patterns for the four recorded interferograms

$$
\begin{aligned}
& I_{1}(x, y)=I_{o}(x, y)(1+v(x, y) \cos (\Delta \phi(x, y))) \\
& I_{2}(x, y)=I_{o}(x, y)\left(1+v(x, y) \cos \left(\Delta \phi(x, y)+\frac{\pi}{2}\right)\right)
\end{aligned}
$$




$$
\begin{aligned}
& I_{3}(x, y)=I_{o}(x, y)(1+v(x, y) \cos (\Delta \phi(x, y)+\pi)) \\
& I_{4}(x, y)=I_{o}(x, y)\left(1+v(x, y) \cos \left(\Delta \phi(x, y)+\frac{3 \pi}{2}\right)\right)
\end{aligned}
$$

The phase difference at each measurement point can then be calculated using

$$
\Delta \phi(x, y)=\tan ^{-1}\left[\frac{I_{4}(x, y)-I_{2}(x, y)}{I_{1}(x, y)-I_{3}(x, y)}\right]
$$

\subsubsection{Fourier Transform Method}

The Fourier Transform Method (FTM) is a widely used full-field optical method for the measurement of 3-D shapes [76-78]. This method was initially developed in order to analyse simple fringe patterns produced by interferometry [79]. It was later applied to the measurement of 3-D shapes with success [80,81]. Standard FTM only requires one frame of the fringe pattern in order to acquire the phase map, making it significantly less sensitive to motion when compared to methods requiring a minimum of three fringe patterns.

A sinusoidal fringe pattern for various optical measurements can be expressed as follows

$$
I(x, y)=a(x, y)+b(x, y) \cos \left[2 \pi f_{0} x+\varphi(x, y)\right]
$$

In this equation, $\varphi(x, y)$ represents the phase that contains the information of interest, $a(x, y)$ represents the unwanted background illumination, $b(x, y)$ represents the local amplitude of the cosine function and refers to the surface reflectivity, and $f_{0}$ represents the carrier frequency. Applying Euler's formula to Eq. 2.21,

$$
I(x, y)=a(x, y)+c(x, y) e^{i 2 \pi f_{0} x}+c^{*}(x, y) e^{-i 2 \pi f_{0} x}
$$

where

$$
c(x, y)=1 / 2 b(x, y) e^{i \varphi(x, y)}
$$

and $*$ denotes a complex conjugate. Using the 1-D Fourier transform with respect to $\mathrm{x}$, Eq. 2.22 gives the following equation:

$$
G\left(f_{x}, y\right)=A\left(f_{x}, y\right)+C^{*}\left(f_{x}+f_{0}, y\right)+C\left(f_{x}-f_{0}, y\right)
$$

where $A\left(f_{x}, y\right)$ and $C^{*}\left(f_{x}+f_{0}, y\right)$ are the 1-D Fourier transforms of $a(x, y)$ and $c(x, y)$ respectively. Assume that $\varphi(x, y), a(x, y)$ and $b(x, y)$ vary very slowly when compared to the carrier frequency $f_{0}$. The three terms in Eq. 2.24 are separated in the spatial frequency domain. The Fourier spectra are shown in Figure 2-9. 

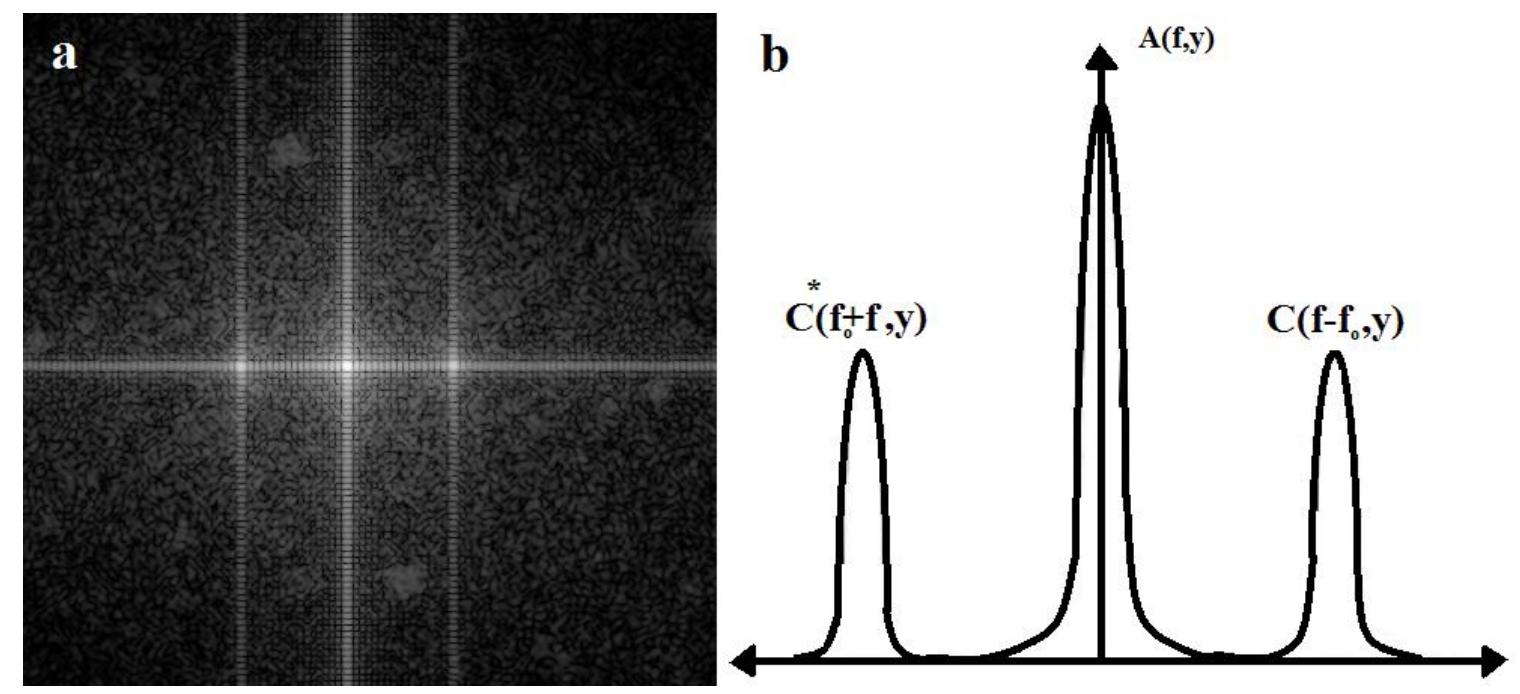

Figure 2-9: a- Fourier spectra image and b- Separated Fourier spectra signal of one fringe pattern.

In order to acquire the desire phase information, the Fourier spectra signals can be separated using the proper design filter. By applying the Inverse Fourier transform to isolate the $C\left(f_{x}+\right.$ $\left.f_{0}, y\right)$ term, the formula becomes

$$
\varphi(x, y)=\operatorname{Im}[\log [c(x, y)]]=\operatorname{Im}\left[\log \left[\frac{1}{2} b(x, y),+i \varphi(x, y)\right]\right]
$$

The phase $\varphi(x, y)$ can be extracted by computing the logarithm of $c(x, y)$, and then isolating the imaginary portion. The phase obtained is wrapped in the $-\pi$ to $\pi$ range. A computer function can be used to conduct the phase unwrapping process and remove the $2 \pi$ discontinuity. Basic knowledge of the Fourier transform and its working principle is essential in order to perform this task.

The Fourier theory is one of the most important tools for digital signal and image processing to analyze the interferograms. The interferograms are usually form by 2-D arrays of pixel values. The image formed is thus discrete, not continuous. Any further processing is applied to the image pixel by pixel. That being said, the Fourier transform can be presented in a continuous or discrete form.

The Fourier transform in a continuous function $f(t)$ of a continuous variable, $t$, is defined as follows: 


$$
F(v)=\int_{-\infty}^{\infty} f(t) e^{-i 2 \pi v t} d t
$$

where $v$ also represents a continuous variable. The Fourier transform is a function of angular frequency $\omega=2 \pi \nu$.

Conversely, given $F(v)$, we can get $f(t)$ back using inverse Fourier transform. The formula is written as follows:

$$
f(t)=\int_{-\infty}^{\infty} F(v) e^{i 2 \pi v t} d v
$$

Therefore, the Fourier transform pair is comprised of Eq. 2.26 and 2.27, indicating that a function can be recovered from its transform. The Fourier transform function of two variables is written as follows

$$
F(u, v)=\int_{-\infty}^{\infty} \int_{-\infty}^{\infty} f(x, y) e^{-i 2 \pi(u x+v y)} d x d y
$$

The inverse Fourier transform of two variables is then obtained using

$$
f(x, y)=\int_{-\infty}^{\infty} \int_{-\infty}^{\infty} F(u, v) e^{i 2 \pi(u x+u v)} d u d v
$$

Digital images can be described using discrete 2-D functions. Since digital images have a finite number of pixels, the discrete Fourier transform (DFT) of a function is taken over the total number of the pixels. The discrete Fourier transform of a function, $f$, of one discrete variable, $m$, by which has $M$ different values is obtained using

$$
F_{m}=\sum_{n=0}^{M-1} f_{n} e^{-i 2 \pi m n / M} \quad m=0,1,2, \ldots, M-1
$$

The inverse discrete Fourier transform (IDFT) is then obtained using

$$
f_{n}=\frac{1}{M} \sum_{m=0}^{M-1} F_{m} e^{i 2 \pi m n / M} \quad n=0,1,2, \ldots, M-1
$$

And finally, the 2-D discrete Fourier transform of a function, $f(x, y)$, of discrete variables $x$, which has $M$ different values, and $y$, which has $N$ different values, is obtained using

$$
F(u, v)=\sum_{x=0}^{M-1} \sum_{y=0}^{N-1} f(x, y) e^{-i 2 \pi\left(\frac{u x}{M}+\frac{v y}{N}\right)}
$$


where, $u=0,1,2, \ldots, M-1$ and $v=0,1,2, \ldots, N-1 . f(x, y)$ can be thought of as a digital image of size $M \times N$ pixels. Its inverse discrete Fourier transform is obtained using

$$
f(x, y)=\frac{1}{M N} \sum_{x=0}^{M-1} \sum_{y=0}^{N-1} F(u, v) e^{i 2 \pi\left(\frac{u x}{M}+\frac{v y}{N}\right)}
$$

where $x=0,1,2, \ldots, M-1$ and $y=0,1,2, \ldots, M-1$. Eq.1.32 and 2.33 constitute the $2-D$ discrete Fourier transform pair.

\subsection{Interference Phase Image Analysis}

The CCD camera records digital images of the interference pattern created by the interference of the object beam and the reference beam. The object beam passing through the sample under investigation will reveal a bigger phase lag than the reference beam. This is because the sample under investigation possesses a higher refractive index than the surrounding medium. The function that defines the intensity distribution of the interferogram is a function of two individual variables that run over the size of the image. The function that describes the image of the intensity of the beam that reaches the CCD camera can be designated as $I_{r}(m, n)$, where $m=0,1 \ldots M-1$ and $n=0,1 \ldots N-1 . M$ and $N$ are the total number of columns and rows of the pixels of the phase image. The function that describes the object beam can be designated as $I_{o}(m, n)$. Then, based on Eq. 2.16

$$
I(m, n)=I_{r}(m, n)+I_{o}(m, n)+2 \sqrt{I_{r}(m, n) I_{o}(m, n)} \cos [\varphi(n, m)]
$$

where $\varphi(n, m)$ represents the interference phase. Eq. 2.18 can then be written as

$$
I(m, n)=A(m, n)+B(m, n) \cos [\varphi(n, m)]
$$

where $B(m, n)$ represents the intensity of the phase image modulated by the cosine of the phase, and $A(m, n)$ represents the background intensity. Since the background intensity is constant, we can re-write $A(m, n)$ as $A$. This background intensity is the average intensity of the whole interferogram;

$$
A=\frac{1}{M N} \sum_{m=0}^{M-1} \sum_{n=0}^{N-1} I(m, n)
$$


Having defined the intensity distribution of our phase image, we can solve the phase distribution of the interferogram. The goal is to obtain $\varphi(m, n)$ from Eq. 2.35 by subtracting Eq. 2.36 from Eq. 2.36. This leaves

$$
I^{*}(m, n)=B(m, n) \cos [\varphi(m, n)]
$$

Now if we use Euler's formula for cosine, i.e.

$$
\cos (\varphi)=\frac{1}{2}\left[e^{i \theta}+e^{-i \theta}\right]
$$

Eq. 2.38 then takes the form

$$
I^{*}(m, n)=\frac{1}{2} B(m, n)\left[e^{i \varphi(m, n)}+e^{-i \varphi(m, n)}\right]
$$

Eq. 2.39 reveals that $I^{*}(m, n)$ is actually an image which is the sum of two complex valued images, $I^{* *}(m, n)$ and $I^{-* *}(m, n)$, that are modulated in opposite directions,

$$
I^{*}(m, n)=I^{* *}(m, n)+I^{-* *}(m, n)
$$

where

$$
I^{* *}(m, n)=\frac{1}{2} B(m, n) e^{i \varphi(m, n)}
$$

and

$$
I^{-* *}(m, n)=\frac{1}{2} B(m, n) e^{-i \varphi(m, n)}
$$

Since the phase can be obtained using Eq. 2.41a or 2.41b, the next step is to remove either $I^{* *}(m, n)$ or $I^{-* *}(m, n)$ from Eq. 1.40. These two images have opposing spatial frequencies. It is thus simpler to separate them in the frequency domain. In Matlab ${ }^{\circledR}$ we can hide the half of the Fourier transformed image $I^{*}(m, n)$ that contains the negative frequencies. Once this is done we need to do an inverse Fourier transform to obtain $I^{* *}(m, n)$. Having separated $I^{* *}(m, n)$ from $I^{-* *}(m, n)$, it is now simple to calculate the wrapped phase using the arctan function

$$
\varphi(m, n)=\arctan \left[\frac{\operatorname{Im}\left(I^{* *}(m, n)\right)}{\operatorname{Re}\left(I^{* *}(m, n)\right)}\right]
$$

where, $\operatorname{Im}\left(I^{* *}(m, n)\right)$ and $\operatorname{Re}\left(I^{* *}(m, n)\right)$ represent the imaginary and real portions of $I^{* *}(m, n)$. As discussed at the beginning of this chapter, the arctan function provides a wrapped phase image. 
A common interferometric measurement process involves causing an interference fringe, converting the fringe pattern into phases or frequencies and computing physical quantities such as surface shape, surface roughness, volumetric thickness and distance. There are three important optical parameters for each ocular component: thickness, surface profile and refractive index. 


\section{CHAPTER 3 PREDICTION AND EXPERIMENTAL MEASUREMENT OF REFRACTIVE INDEX IN TERNARY HYDROCARBON MIXTURES}

This chapter is based on the following published paper:

Yahya M., Saghir M. Z. "Prediction and Experimental Measurements of Refractive Index in Ternary Hydrocarbon Mixtures”. J. Chem. Eng. Data, volume 60, issue 8, pp. 2329-2342, 2015.

\subsection{Introduction}

Quantitative measurements of the optical properties of liquid mixtures can be obtained using the coherent light source interference phenomenon. This method is widely used in optical digital interferometry (ODI) [12-16], refractometry [15,17,18], optical beam deflection (OBD) [19-21], and other techniques [22-25].

The refractive index is consider one of the most important optical properties of a transparent medium and is highly affected by concentration, temperature, wavelength and pressure [1-4]. Knowledge of the temperature and concentration derivative of the refractive indices of liquid mixtures [known as Contrast Factor (CF)] plays a significant role in science and engineering designs. The refractive index of mixtures allows for quick diagnostic information regarding the composition of chemical substances. This includes purity, dielectric permeability, the boiling point of a medium, and other thermodynamic properties such as thermal diffusion (Soret effect) [82-84]. Precise measurements of the refractive index are thus critical in science and technology $[10,11]$. The present study provides precise experimental measurements of the refractive indices of three hydrocarbon components: 1,2,3,4-Tetrtahydronaphtalenene (THN), Isobutylbenzen (IBB), and Dodecane $\left(n \mathrm{C}_{12}\right)$.

An extensive literature search revealed that the refractive indices for these proposed ternary mixtures are missing or limited to only a few mixtures. These components have been selected because they are simplified multicomponent substances of hydrocarbon in natural crude oil reservoirs [30] and of great interest in the Differential Scanning Calorimetry (DSC) and Influence of Vibrations on diffusion in Liquid (IVIDIL) experiments currently taking place (on ground and in space) at the International Space Station (ISS) [31]. Although the properties of pure substances and their binary mixtures can be found in the literature $[5,15]$, there is very little information available regarding ternary mixtures. In the present study, we measured the refractive index of different binary and ternary compositions $\left(n_{T H N}\right)_{I B B}\left|n C_{12}, \quad\left(n_{I B B}\right)_{n C_{12}}\right| T H N$ 
and $\left(n_{n C_{12}}\right)_{T H N} \mid I B B$ and their pure components over a wide range of temperatures and wavelengths.

In addition to experimental measurement, the refractive indices of transparent mixtures can be predicted using 'mixing rule' equations. These equations make use of the refractive index values of the pure components of the mixture. The most widely recognized theoretical mixing rules used for predicting the refractive index of liquid mixtures are those of Lorentz-Lorenz and Weiner. Other mixing rules have been developed by scientists such as Heller and GladstoneDale. Research has been conducted to validate these mixing equations [2, 85-87], and some of these mixing rules were used to validate the experimental data obtained in this study.

In the following section we present the materials and sample preparation methods used in this study, as well as an abbreviated description of the technique used to measure the refractive index. We will present of our refractive index calculations using experimental methods as well as the proposed correlations in the discussion section.

\subsection{Experimental}

The pure substance 1,2,3,4-tetra-hydro-naphthalene (THN; two ring hydrocarbon with chemical formula $\mathrm{C}_{10} \mathrm{H}_{12}$ ), Isobutybenzene (IBB; one ring hydrocarbon with chemical formula $\left.\mathrm{C}_{4} \mathrm{H}_{8}\right)$ and Dodecane $\left(n \mathrm{C}_{12}\right.$; alkane hydrocarbon with chemical formula $\left.\mathrm{C}_{12} \mathrm{H}_{26}\right)$ were supplied by Acros Organics®. The stated purity of these liquids was between 98 and $99 \%$ and the liquids were used to prepare the studied mixtures.

Sixty-three compositions (thirty six ternaries and twenty seven binaries) were prepared. This was done using three pure substances from the pre-prescribed mass fractions of pure components mixed together for a net mass of $5 \mathrm{~g}(5 \mathrm{ml})$, charged in leak proof caps. The mass fractions varied with a sampling rate of $10 \%$ for each component. The mass was measured using a Denver Instrument ${ }^{\circledR}$ SI-234 electronic balance, with an estimated error of $\pm 1 \times 10^{-3} \mathrm{~g} / 5 \mathrm{~g}$. The mixtures were prepared in a fume hood lab bench at a room temperature of $296.15 K$ with $\pm 2 K$ stability. In order to minimize any error due to substance evaporation, we added the less volatile component (i.e., the alkane $n \mathrm{C}_{12}$ with higher molecular weight), followed by the corresponding amount of the second substance. The mixtures were used immediately following preparation in order to minimize evaporation and reduce measurement error. 
The refractive indices of the prepared compositions were measured using an automatic Anton Paar® WR Refractometer. This device uses reflected light rather than transmitted light to measure the refractive index. During testing, the sample is placed in a sample mold on top of the measuring YAG prism (Yttrium-Aluminum-Garnet) that irradiates from different angles via an LED light. A high-power LED was used as the light source along with an interference filter yielding a wavelength error of $0.1 \mathrm{~nm}$. At the interface between the sample and the prism, the incident beam is either refracted into the sample or reflected back into the prism. The reflected beam is then detected by a sensor array. From this, the critical angle for total reflection is calculated and used to determine the refractive index of the sample based on Snell's law [88]. The temperature of the prism was controlled by a Peltier thermostat with a temperature stability of $\pm 2 \times 10^{-3} \mathrm{~K}$ within the working range. The instrument accuracy for the refractive index value was $\pm 4 \times 10^{-5}$. The average standard deviation of the mean "standard uncertainty" of the instrument, expressed by $u_{i}=\frac{a}{\sqrt{3}}$, (where $a$ represent the semi-range between the upper and lower limits) were estimated to be $4.62 \times 10^{-5}$.

The instrument was calibrated before each series of measurements using double distilled water with a specific conductance of $2.0 \mu \mathrm{mho} / \mathrm{cm}$ at $296.15 \mathrm{~K}$. The calibrations were conducted in accordance with a specifications table supplied by the refractometer manufacturer. The instrument was cleaned with Acetone after each calibration and sample measurement and dried with lab wipes. All measurements were conducted at atmospheric pressure. In order to cover the whole concentration of the studied mixtures of $\left(n_{T H N}\right)_{I B B}\left|n C_{12}, \quad\left(n_{I B B}\right)_{n C_{12}}\right| T H N$ and $\left(n_{n C_{12}}\right)_{T H N} \mid I B B$, sixty six samples (36 ternaries +27 binaries +3 pure substances) of THN concentration (with an average mass fraction between 0 and 1, corresponding to mass fraction changes of IBB and $n \mathrm{C}_{12}$ ) were prepared. The mass fractions were increased in increments of 0.1.The refractive indices were measured at atmospheric pressure for five different wavelengths $(436.1,513.9,589.3,632.9$ and 657.2) $\mathrm{nm}$ and six different temperatures $\mathrm{T}=(293.15$ to 318.15$)$ $K$. The total number of measurements was 1980 (66 samples $\times 6$ different temperatures $\times 5$ wavelengths). The mixtures were placed on a magnetic stirrer for several minutes prior to measurement in order to ensure the homogeneity of the mixture.

All measurements were repeated three times within the same condition at the same atmospheric pressure $P=0.1 \mathrm{MPa}$ in order to increase measurement accuracy. The average 
standard uncertainty of the repeated measurements $u_{r}=\frac{S D}{\sqrt{n}}(S D$ represents the standard deviation for the number of measurements $n$ ), calculated based on the mean values of the repeatability of refractive index measurements was $3.527 \times 10^{-4}$.

The verification of the accuracy and the repeatability of the refractive index determination was accomplished by calculating the average expanded uncertainty using the given expression $U=k u_{c}$ where $u_{c}=u_{r}+u_{i}$ is the total standard uncertainty. The estimated average expanded uncertainty was $1.197 \times 10^{-3}$, calculated based the coverage factor $k=2$, providing a level of confidence of approximately $95 \%$.

\subsection{Results and Discussion}

The refractive indices of three hydrocarbon mixtures were measured using the Abbemat Refractometer technique for five different wavelengths $\lambda=(436.1,513.9,589.3,632.9$ and 657.2) $n m$ and six different temperatures $\mathrm{T}=(293.15$ to 318.15$) \mathrm{K}$. Sixty three samples were prepared at an accuracy of $\pm 1 \times 10^{-3} \mathrm{~g} / 5 \mathrm{~g}$. The three pure components of each mixture were also examined at isobaric condition.

The experimental measurements obtained revealed a linear relationship between refractive index and concentration and temperature, as well as a non-linear relationship between refractive index and wavelength [89]. This behaviour was observed in the ternary mixtures as well as the binaries and pure substances. The experimental values of the refractive indices of the THN, IBB, and $n \mathrm{C}_{12}$ mixtures at various wavelengths and temperatures can be found in Tables 3.1, and the rest of the tables attached in the appendix 3.1-3.4. Since all of the investigated mixtures revealed the same behaviour as a function of temperature and wavelength, only some of the data was selected to illustrate our findings in the discussion section. The following figures and theoretical calculations were conducted using the selected data.

The refractive index of the ternary systems under study at a wavelength of $657.2 \mathrm{~nm}$ is provided in Table 3.1 and used in the discussion section. Figure 3-1 illustrates the linear interpolation of the THN-IBB mass fractions normalized by a mean of 0.333 , standard deviation of 0.270 and the sum of the squared errors of $2.958 \times 10^{-31}$. 


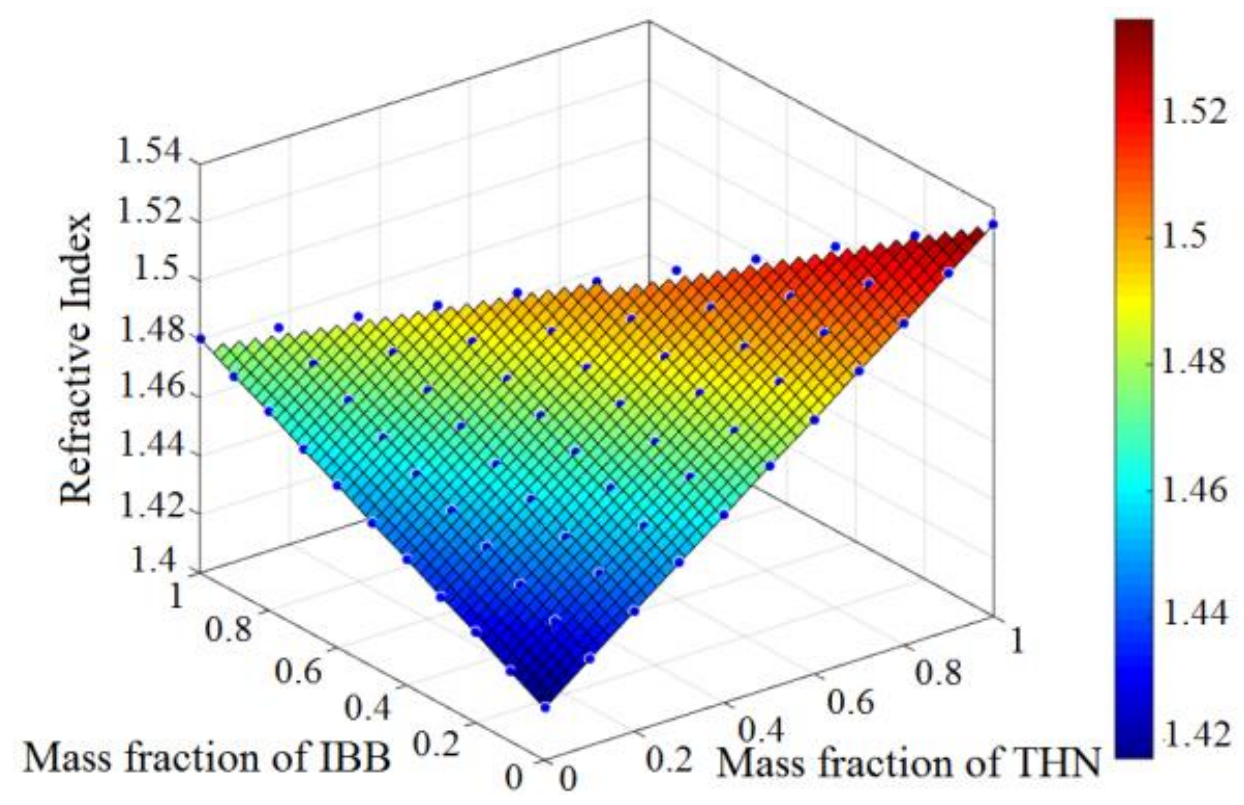

Figure 3-1: Refractive index of $\left(\mathrm{n}_{\mathrm{THN}}\right) \mathrm{IBB} \mid n \mathrm{C}_{12}$ at wavelengths $657.2 \mathrm{~nm}$ and $\mathrm{T}=298.15$ $K$ as function of ternary system composition.

A closer look at the tables reveals that the refractive index of the mixtures and the pure components increases as the temperature decreases. This can be attributed to variations in density caused by the temperature variation. Analysis of Table 3-1 reveals that the refractive index values decrease in a linear fashion when the temperature increases by $25 \mathrm{~K}$. For instance, the refractive index of $\left(n_{I B B}\right)_{n C_{12}} \mid T H N=0.1$ decreased by an average of 0.992 when the temperature increased by 1.085. The investigation of the concentration derivative of the refractive index also reveals a linear increase in refractive indices with increases in concentration.

Table 3.1: Refractive indices and temperature coefficients of $\mathrm{THN}, \mathrm{IBB}$, and $n \mathrm{C}_{12}$ at temperatures of $\mathrm{T}=$ (293.15 to 318.15) $\mathrm{K}$ and wavelength of $657.2 \mathrm{~nm}$ at atmospheric pressure $\mathrm{P}=0.1 \mathrm{MPa}^{\mathrm{a}}$.

\begin{tabular}{|c|c|c|c|c|c|c|c|c|c|}
\hline \multicolumn{3}{|c|}{ Mole Fraction } & \multicolumn{6}{|c|}{ Wavelength $\lambda=657.2 \mathrm{~nm}$} & \multirow{2}{*}{$(\operatorname{dn} / d T)$} \\
\hline THN & IBB & $n \mathrm{C}_{12}$ & 293.15 & 298.15 & 303.15 & 308.15 & 313.15 & 318.15 & \\
\hline 0 & 0 & 1 & 1.41993 & 1.41777 & 1.41564 & 1.4135 & 1.41134 & 1.4092 & $-4.29 \mathrm{E}-04$ \\
\hline 0 & 1 & 0 & 1.48286 & 1.48035 & 1.47798 & 1.47555 & 1.47309 & 1.47063 & $-4.88 \mathrm{E}-04$ \\
\hline \multirow[t]{2}{*}{1} & 0 & 0 & 1.53694 & 1.53452 & 1.53208 & 1.52971 & 1.52732 & 1.52495 & $-4.80 \mathrm{E}-04$ \\
\hline & \multicolumn{8}{|c|}{ Binary mixtures of $\left(\mathrm{n}_{\mathrm{IBB}}\right) n \mathrm{C}_{12}$} & \\
\hline 0 & 0.9014 & 0.0986 & 1.47491 & 1.47249 & 1.47001 & 1.46758 & 1.46515 & 1.46262 & $-4.91 \mathrm{E}-04$ \\
\hline 0 & 0.8024 & 0.1976 & 1.46776 & 1.46548 & 1.46299 & 1.46065 & 1.45817 & 1.45594 & $-4.76 \mathrm{E}-04$ \\
\hline
\end{tabular}




\begin{tabular}{|c|c|c|c|c|c|c|c|c|c|}
\hline 0 & 0.7032 & 0.2968 & 1.46085 & 1.45863 & 1.45625 & 1.45387 & 1.45159 & 1.44935 & $-4.63 \mathrm{E}-04$ \\
\hline 0 & 0.6036 & 0.3964 & 1.45427 & 1.45198 & 1.44965 & 1.44715 & 1.44496 & 1.44244 & $-4.73 E-04$ \\
\hline 0 & 0.5038 & 0.4962 & 1.44849 & 1.44578 & 1.44358 & 1.44115 & 1.43882 & 1.43665 & $-4.71 \mathrm{E}-04$ \\
\hline 0 & 0.4036 & 0.5964 & 1.44269 & 1.44009 & 1.43801 & 1.43554 & 1.43304 & 1.43091 & $-4.72 \mathrm{E}-04$ \\
\hline 0 & 0.3032 & 0.6968 & 1.43659 & 1.43417 & 1.4321 & 1.42984 & 1.42761 & 1.42574 & $-4.35 \mathrm{E}-04$ \\
\hline 0 & 0.2024 & 0.7976 & 1.43084 & 1.42867 & 1.42618 & 1.42395 & 1.42149 & 1.41979 & $-4.52 \mathrm{E}-04$ \\
\hline 0 & 0.1014 & 0.8986 & 1.42541 & 1.42267 & 1.42052 & 1.41824 & 1.41612 & 1.41386 & $-4.55 \mathrm{E}-04$ \\
\hline \multicolumn{10}{|c|}{ Binary mixtures of $\left(n_{\mathrm{THN}}\right) n \mathrm{C}_{12}$} \\
\hline 0.1014 & 0 & 0.8986 & 1.42878 & 1.42675 & 1.42434 & 1.42224 & 1.41998 & 1.41814 & $-4.32 \mathrm{E}-04$ \\
\hline 0.2024 & 0 & 0.7976 & 1.43896 & 1.43623 & 1.434 & 1.4316 & 1.42919 & 1.42702 & $-4.76 \mathrm{E}-04$ \\
\hline 0.3032 & 0 & 0.6968 & 1.44835 & 1.44627 & 1.44387 & 1.44127 & 1.439 & 1.43701 & $-4.63 \mathrm{E}-04$ \\
\hline 0.4036 & 0 & 0.5964 & 1.45921 & 1.45678 & 1.45441 & 1.45195 & 1.44965 & 1.44718 & $-4.80 \mathrm{E}-04$ \\
\hline 0.5038 & 0 & 0.4962 & 1.47078 & 1.46851 & 1.46615 & 1.46385 & 1.46161 & 1.45934 & $-4.58 \mathrm{E}-04$ \\
\hline 0.6036 & 0 & 0.3964 & 1.48194 & 1.47967 & 1.47729 & 1.47498 & 1.47245 & 1.47014 & $-4.74 \mathrm{E}-04$ \\
\hline 0.7032 & 0 & 0.2968 & 1.4944 & 1.49181 & 1.48949 & 1.48694 & 1.48468 & 1.48216 & $-4.87 \mathrm{E}-04$ \\
\hline 0.8024 & 0 & 0.1976 & 1.50801 & 1.50529 & 1.50284 & 1.50013 & 1.49759 & 1.49507 & $-5.17 \mathrm{E}-04$ \\
\hline 0.9014 & 0 & 0.0986 & 1.52229 & 1.5199 & 1.51741 & 1.51517 & 1.51275 & 1.51044 & $-4.74 \mathrm{E}-04$ \\
\hline \multicolumn{10}{|c|}{ Binary mixtures of $\left(\mathbf{n}_{\mathrm{THN}}\right)$ IBB } \\
\hline 0.1014 & 0.8986 & 0 & 1.48749 & 1.48512 & 1.48275 & 1.48038 & 1.47807 & 1.47562 & $-4.74 \mathrm{E}-04$ \\
\hline 0.2024 & 0.7976 & 0 & 1.49254 & 1.49029 & 1.48792 & 1.48538 & 1.48312 & 1.48056 & $-4.80 \mathrm{E}-04$ \\
\hline 0.3032 & 0.6968 & 0 & 1.49852 & 1.49594 & 1.49332 & 1.49085 & 1.48866 & 1.48595 & $-4.98 \mathrm{E}-04$ \\
\hline 0.4036 & 0.5964 & 0 & 1.50384 & 1.50135 & 1.49885 & 1.49669 & 1.49418 & 1.49181 & $-4.79 \mathrm{E}-04$ \\
\hline 0.5038 & 0.4962 & 0 & 1.50967 & 1.50706 & 1.50461 & 1.50224 & 1.49947 & 1.49685 & $-5.10 \mathrm{E}-04$ \\
\hline 0.6036 & 0.3964 & 0 & 1.51394 & 1.51205 & 1.50983 & 1.50738 & 1.50491 & 1.50222 & $-4.71 \mathrm{E}-04$ \\
\hline 0.7032 & 0.2968 & 0 & 1.52005 & 1.51776 & 1.51528 & 1.51272 & 1.51025 & 1.50758 & $-5.00 \mathrm{E}-04$ \\
\hline 0.8024 & 0.1976 & 0 & 1.52522 & 1.52302 & 1.52043 & 1.51825 & 1.51573 & 1.51388 & $-4.61 \mathrm{E}-04$ \\
\hline 0.9014 & 0.0986 & 0 & 1.53141 & 1.52941 & 1.52666 & 1.52447 & 1.52168 & 1.51897 & $-5.00 \mathrm{E}-04$ \\
\hline \multicolumn{10}{|c|}{ Ternary mixtures of $\mathrm{THN}|\mathrm{IBB}| n \mathrm{C}_{12}$} \\
\hline 0.122 & 0.1202 & 0.7578 & 1.43495 & 1.43274 & 1.43055 & 1.42835 & 1.42608 & 1.42394 & $-4.41 \mathrm{E}-04$ \\
\hline 0.119 & 0.2344 & 0.6466 & 1.44064 & 1.43843 & 1.43619 & 1.4339 & 1.43163 & 1.42942 & $-4.50 \mathrm{E}-04$ \\
\hline 0.2376 & 0.117 & 0.6454 & 1.44496 & 1.44265 & 1.4405 & 1.43845 & 1.43609 & 1.43366 & $-4.47 \mathrm{E}-04$ \\
\hline 0.1161 & 0.3431 & 0.5408 & 1.44676 & 1.44457 & 1.44221 & 1.44004 & 1.43768 & 1.43547 & $-4.53 \mathrm{E}-04$ \\
\hline 0.3472 & 0.114 & 0.5389 & 1.4553 & 1.45302 & 1.45073 & 1.44845 & 1.44623 & 1.4439 & $-4.55 \mathrm{E}-04$ \\
\hline 0.1134 & 0.4467 & 0.44 & 1.45294 & 1.45055 & 1.44829 & 1.44585 & 1.44367 & 1.4411 & $-4.70 \mathrm{E}-04$ \\
\hline 0.2264 & 0.3344 & 0.4392 & 1.45719 & 1.45507 & 1.45257 & 1.45022 & 1.448 & 1.44582 & $-4.59 \mathrm{E}-04$ \\
\hline 0.339 & 0.2226 & 0.4385 & 1.46173 & 1.4595 & 1.45711 & 1.45486 & 1.45249 & 1.45024 & $-4.61 \mathrm{E}-04$ \\
\hline 0.4512 & 0.1111 & 0.4377 & 1.46624 & 1.46399 & 1.46158 & 1.45935 & 1.45701 & 1.45474 & $-4.61 \mathrm{E}-04$ \\
\hline
\end{tabular}




\begin{tabular}{|c|c|c|c|c|c|c|c|c|c|}
\hline 0.1107 & 0.5454 & 0.3438 & 1.45948 & 1.45711 & 1.45475 & 1.45239 & 1.44998 & 1.44758 & $-4.76 E-04$ \\
\hline 0.3311 & 0.3262 & 0.3427 & 1.46859 & 1.46621 & 1.46376 & 1.46153 & 1.45905 & 1.45688 & $-4.70 \mathrm{E}-04$ \\
\hline 0.5501 & 0.1084 & 0.3416 & 1.47777 & 1.47531 & 1.47301 & 1.47054 & 1.46828 & 1.46581 & $-4.76 \mathrm{E}-04$ \\
\hline 0.1082 & 0.6397 & 0.252 & 1.46601 & 1.46381 & 1.46139 & 1.45911 & 1.45675 & 1.45458 & $-4.61 \mathrm{E}-04$ \\
\hline 0.2161 & 0.5322 & 0.2516 & 1.47085 & 1.46844 & 1.46617 & 1.46378 & 1.46154 & 1.45922 & $-4.64 \mathrm{E}-04$ \\
\hline 0.3237 & 0.4251 & 0.2512 & 1.47548 & 1.47318 & 1.47083 & 1.4684 & 1.46598 & 1.46361 & $-4.76 \mathrm{E}-04$ \\
\hline 0.4309 & 0.3183 & 0.2508 & 1.48028 & 1.478 & 1.47544 & 1.47314 & 1.4708 & 1.46831 & $-4.79 \mathrm{E}-04$ \\
\hline 0.5377 & 0.2119 & 0.2504 & 1.48511 & 1.48283 & 1.4804 & 1.47815 & 1.47582 & 1.47351 & $-4.64 \mathrm{E}-04$ \\
\hline 0.6442 & 0.1058 & 0.25 & 1.48985 & 1.48755 & 1.48501 & 1.48268 & 1.48025 & 1.47787 & $-4.81 E-04$ \\
\hline 0.1059 & 0.7298 & 0.1643 & 1.47314 & 1.47086 & 1.46835 & 1.46605 & 1.46366 & 1.46137 & $-4.73 \mathrm{E}-04$ \\
\hline 0.3166 & 0.5197 & 0.1638 & 1.48285 & 1.48044 & 1.47798 & 1.47571 & 1.47329 & 1.471 & $-4.74 \mathrm{E}-04$ \\
\hline 0.5259 & 0.3108 & 0.1633 & 1.4928 & 1.49046 & 1.48811 & 1.48558 & 1.48339 & 1.48089 & $-4.76 \mathrm{E}-04$ \\
\hline 0.734 & 0.1033 & 0.1628 & 1.50293 & 1.5005 & 1.49808 & 1.4958 & 1.49344 & 1.49113 & $-4.71 \mathrm{E}-04$ \\
\hline 0.1036 & 0.8161 & 0.0804 & 1.48044 & 1.47803 & 1.4755 & 1.47309 & 1.47068 & 1.46815 & $-4.91 \mathrm{E}-04$ \\
\hline 0.2068 & 0.7129 & 0.0803 & 1.48564 & 1.48311 & 1.48069 & 1.47814 & 1.47587 & 1.47322 & $-4.94 \mathrm{E}-04$ \\
\hline 0.3097 & 0.6101 & 0.0801 & 1.49051 & 1.48809 & 1.48564 & 1.48318 & 1.48087 & 1.47832 & $-4.86 \mathrm{E}-04$ \\
\hline 0.4123 & 0.5077 & 0.08 & 1.4953 & 1.49284 & 1.49032 & 1.48778 & 1.48538 & 1.48287 & $-4.98 \mathrm{E}-04$ \\
\hline 0.5146 & 0.4055 & 0.0799 & 1.50063 & 1.49836 & 1.49568 & 1.49328 & 1.49061 & 1.48842 & $-4.95 \mathrm{E}-04$ \\
\hline 0.6166 & 0.3037 & 0.0798 & 1.50601 & 1.50341 & 1.50104 & 1.49875 & 1.49624 & 1.49375 & $-4.86 \mathrm{E}-04$ \\
\hline 0.7182 & 0.2021 & 0.0796 & 1.51153 & 1.50897 & 1.50657 & 1.5043 & 1.50154 & 1.49914 & $-4.94 \mathrm{E}-04$ \\
\hline 0.8196 & 0.1009 & 0.0795 & 1.51668 & 1.5144 & 1.51197 & 1.50969 & 1.50734 & 1.50496 & $-4.69 \mathrm{E}-04$ \\
\hline 0.2318 & 0.2284 & 0.5398 & 1.45024 & 1.44795 & 1.44569 & 1.44345 & 1.44115 & 1.4389 & $-4.53 \mathrm{E}-04$ \\
\hline 0.6301 & 0.2069 & 0.163 & 1.49681 & 1.49436 & 1.49185 & 1.48946 & 1.48703 & 1.48441 & $-4.94 \mathrm{E}-04$ \\
\hline 0.2114 & 0.6246 & 0.1641 & 1.47723 & 1.47483 & 1.47244 & 1.47012 & 1.46771 & 1.46525 & $-4.78 \mathrm{E}-04$ \\
\hline 0.2211 & 0.4356 & 0.3433 & 1.46325 & 1.46083 & 1.45834 & 1.45599 & 1.45362 & 1.45129 & $-4.79 \mathrm{E}-04$ \\
\hline 0.4408 & 0.2171 & 0.3421 & 1.47237 & 1.47013 & 1.46781 & 1.46545 & 1.46296 & 1.46082 & $-4.66 \mathrm{E}-04$ \\
\hline 0.4214 & 0.4151 & 0.1635 & 1.48711 & 1.48474 & 1.48245 & 1.48011 & 1.47779 & 1.47544 & $-4.66 \mathrm{E}-04$ \\
\hline
\end{tabular}

${ }^{a}$ The reported average expanded uncertainty $U=1.197 \times 10^{-3}$ was calculated based on the coverage factor $k=2$, providing a level of confidence of approximately $95 \%$.

The average standard deviation comparisons of the temperature and concentration coefficients of the refractive index with available literature $[5,15]$ were presented in table 3.3 .

As mentioned earlier, the prepared mixtures were varied by intervals of $10 \%$ with each pure component. For example, for the $\left(n_{I B B}\right)_{n C_{12}} \mid T H N=0.1$ mixture, the IBB molar fraction increased greatly (from 0 to 0.9 ), while the corresponding molar fraction of $n C_{12}$ decreased (from 1 to 0 ). Consequently, the refractive index shows an average increase of approximately 
1.039. We found similar variations in the temperature and concentration coefficients of refractive index $d n / d T$ and $d n / d c$ for all other systems under investigation.

For a better representation of the experimental results, the refractive index concentration and contrast factor temperature coefficients of the investigated mixtures (over the whole composition at some wavelengths) at a temperature of $298.15 \mathrm{~K}$ are presented in a ternary diagram. Figure 3-2 provides an illustration of the refractive indices of the ternary mixture plotted against the mass fraction at wavelengths of $657.2,632.9,589.2$, and $513.9 \mathrm{~nm}$, and a set temperature of $298.15 \mathrm{~K}$. Figure 3-3 provides an illustration of the temperature coefficient contour lines plotted at different mass fractions for the same wavelengths and temperature. These diagrams present the compositions of the synthetic mixtures with a mass fraction deviation of $10 \%$ and the contour lines of the measured refractive index. These diagrams can be used to predict the refractive indices and temperature coefficients of any composition mixture of (THN, IBB, $n \mathrm{C}_{12}$ ) by projecting the required concentration onto the corresponding binary axis.

Although we observed a linear relationship between refractive indices and temperature and concentration in all the mixtures under investigation, the results revealed non-linear behaviour of refractive indices with variations in wavelength. This can be attributed to the fact that as the light propagates into the medium, the particles of the light "electromagnetic waves" interact with the electric field of the medium. The higher the frequency of the incoming electromagnetic waves, the more the particles interact with the electric field of the medium, reducing velocity of light in the medium and causing a higher refractive index $n=c / v$. In contrast, the refractive index increases non-linearly as the wavelength decreases. The rate of increase became larger as the wavelength became shorter.

The empirical relationship between the refractive index and wavelength of transparent materials governed by the Cauchy formula [89] was adopted to calculate the refractive index at five different wavelengths. 

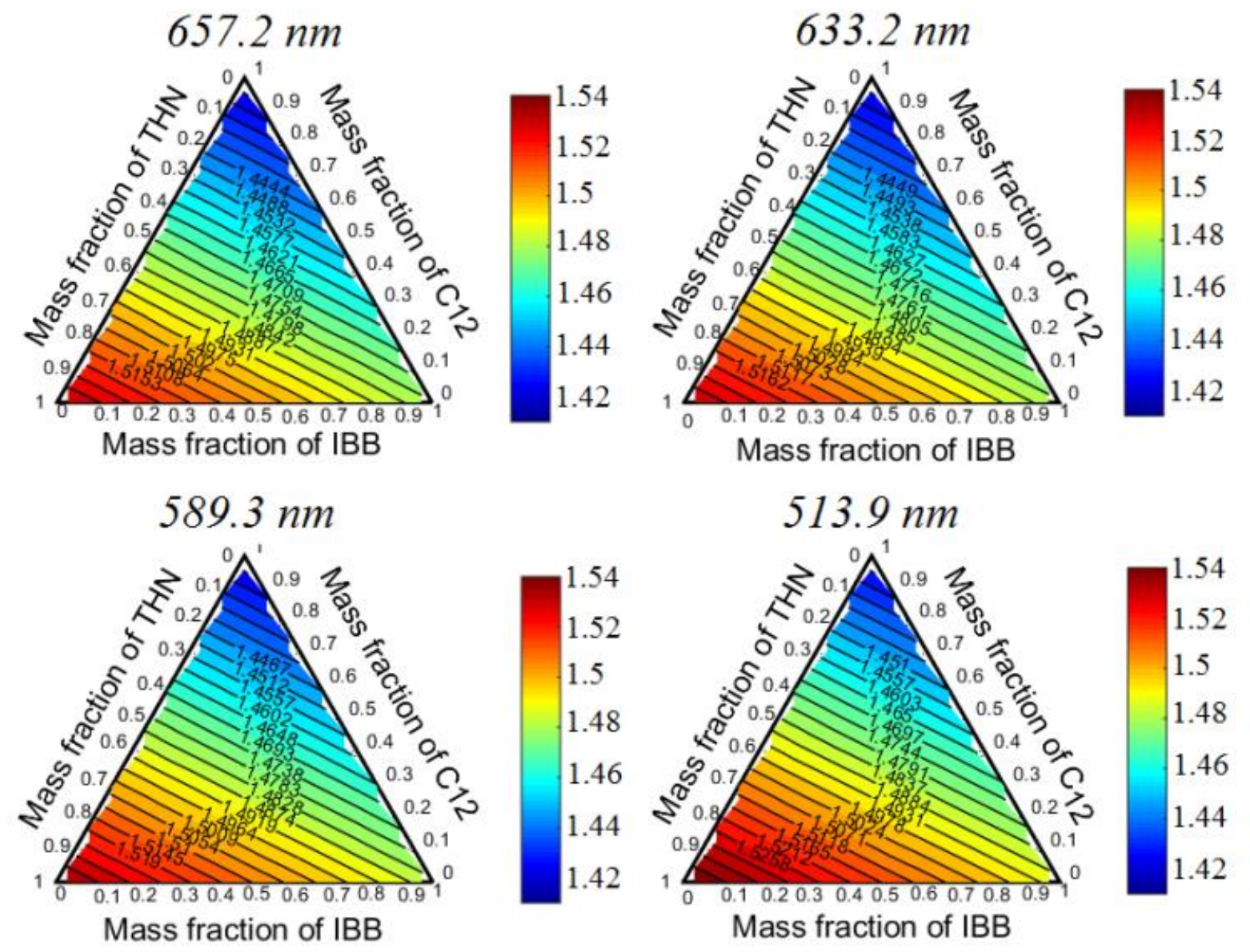

Figure 3-2: Concentration variation of refractive indices of the $\mathrm{THN}-\mathrm{IBB}-n \mathrm{C}_{12}$ mixtures at $\mathrm{T}=$ 298.15 $K$ with respect to mass fraction over the entire concentration, plotted at different wavelengths of $\lambda=(436.1,513.9,589.3,632.9$ and 657.2$) \mathrm{nm}$. 

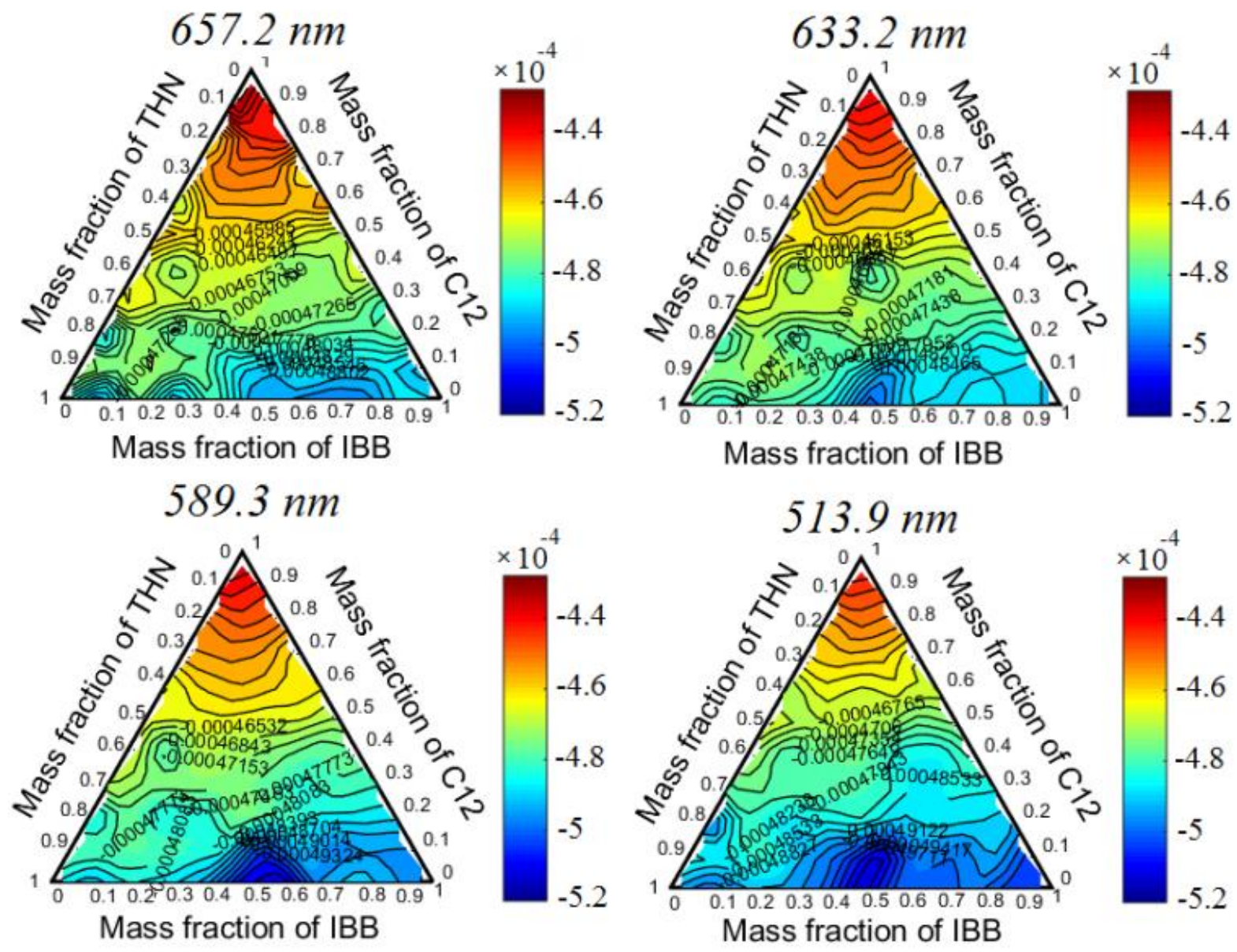

Figure 3-3: Temperature coefficients of refractive indices of the THN-IBB- $n C_{12}$ mixtures at $\mathrm{T}=298.15$ $K$ with respect to mass fraction over the entire concentration, plotted at different wavelengths of $\lambda=$ (436.1, 513.9, 589.3, 632.9 and 657.2) $\mathrm{nm}$. 
The general form of Cauchy's equation is:

$$
\mathrm{n}_{\lambda_{\mathrm{i}}}=\mathrm{A}+\mathrm{B} / \lambda_{\mathrm{i}}{ }^{2}+\mathrm{C} / \lambda_{\mathrm{i}}{ }^{4}+\mathrm{D} / \lambda_{\mathrm{i}}{ }^{6}+\mathrm{E} / \lambda_{\mathrm{i}}{ }^{8}+\mathrm{F} / \lambda_{\mathrm{i}}{ }^{10}
$$

where $n_{\lambda_{i}}$ represents the refractive index at $\lambda_{i}$ wavelength, \& $\mathrm{A}$ to $\mathrm{F}$ represent coefficients that can be determined for given mixtures at known wavelengths. For example, the coefficients A to $\mathrm{F}$ of the pure substance THN at $298.15 \mathrm{~K}$ are $1.6167,-4 \times 10^{-5}, 5.2362 \times 10^{10},-1.0009 \times 10^{16}$ and $7.0019 \times 10^{20}$ respectively. The same procedure was applied to all of the mixtures under study. The relationship between the refractive index and wavelength of ternary mixture $\left(n_{I B B}\right)_{n C_{12}} \mid$ THN $=0.1$ is illustrated in Figure 3-4. The measured values were also verified using Matlab ${ }^{\circledR}$ quadratic polynomial fitting functions where the average fit was $7.105 \times 10^{-16}$.

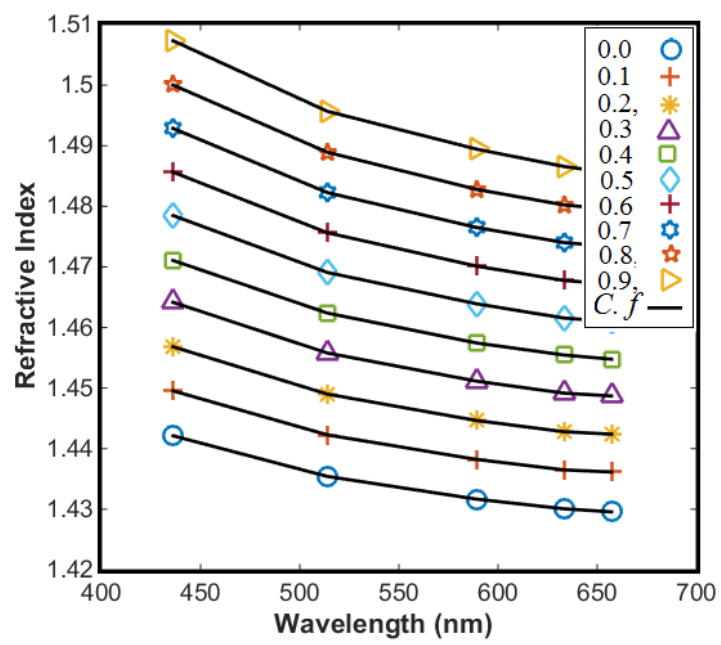

Figure 3-4: Refractive index with respect to the wavelength of the ternary mixture $\left(\mathrm{n}_{\mathrm{IBB}}\right) n \mathrm{C}_{12} \mid \mathrm{THN}=0.1$, at $\mathrm{T}=298.15 \mathrm{~K}$ and different concentrations of IBB, $C$. $f$ - is the Cauchy formula.

\subsection{Mathematical Correlation}

Reliable refractive index data requires accurate experimental measurements. Because the refractive index is correlated with temperature, concentration and wavelength, the development of an empirical model may be useful when conducting research to predict the refractive index of any binary or ternary mixture of THN-IBB- $n \mathrm{C}_{12}$. To the best of our knowledge, no study has evaluated an empirical correlation based on experimental measurements of refractive indices across the entire concentration of the mixtures under study. The experimental data obtained from the measurement of the refractive index of the sixty six samples investigated in this study has 
contributed to the creation of an empirical correlation. The proposed correlation can be used to predict the refractive index of any composition of $\mathrm{THN}-\mathrm{IBB}-n \mathrm{C}_{12}$ mixtures as a function of temperature, concentration and wavelength. The estimated coefficients of the proposed correlation, given in Eq 2, were obtained using multi-input regression analysis and analysis of variance (ANOVA) using MatLab ${ }^{\circledR}$. In this correlation, $C$ represents the mass fraction of the pure components of THN-IBB- $n \mathrm{C}_{12}$, the temperature is in Kelvin and the wavelength is in $\boldsymbol{n m}$.

$$
n=\left(1.72186 T H N+1.67451 I B B+1.60630 n C_{12}\right)-4.798 \times 10^{-4} T-8.256 \times 10^{-5} \lambda
$$

Table 3.2, shows the estimated coefficients and the regression statistics. In this table, the Mean squares represent an estimate of population variance that can be calculated by dividing the corresponding sum of squares by the degrees of freedom. The Standard error represents the square root of the residual mean square using only the coefficient for that row and t-Stat is the coefficient of the variable divided by the standard error for that variable.

In order to evaluate the experimental and predicted refractive index using the proposed correlation, $\mathrm{Eq} 3.3$ was used to compute the corresponding calculated value of the refractive index based on the known refractive index of the pure components of the mixtures under consideration.

$$
\Delta n=n-\sum_{i=1}^{N} \varphi_{i} n_{i}
$$

In this equation, $n$ represents the refractive index of the mixture, $n_{i}$ represents the refractive of the pure component $i$, and $\varphi_{i}$ is the volume fraction of $i^{\text {th }}$ component that can be obtained by the relation.

$$
\varphi_{i}=x_{i} V / \sum_{i=1}^{N} x_{i} V_{i}
$$

where $V$ represents the total mixture volume and $V_{i}$ represents the volume and $x_{i}$ is the mole fraction of the $i^{\text {th }}$ component, in which the mole fraction is the number of moles of the target substance divided by the total number of moles involved. $N$ represents the number of components in the mixture [34,36,89-93].

The results revealed substantial agreement between the experimental data with the obtained values using the proposed correlation and the values calculated using Eq. 3.4 with an average absolute error of $1.55 \times 10^{-3}$ and $2.50 \times 10^{-3}$ respectively. This analysis confirmed the 
accuracy and reliability of the proposed correlation in predicting the refractive index of any concentration of THN-IBB- $n \mathrm{C}_{12}$ mixtures. This remains true for the entire range of wavelengths and temperatures that were studied.

For verification purposes, some of the experimental data were compared with the corresponding values found in the literature [15,5]. The summary of the standard deviation comparisons are provided in Table 3.3 The accuracy of the experimental refractive index measurement was in the third order of magnitude. For instance, at a wavelength of $657 \mathrm{~nm}$ and temperature of $298.15 \mathrm{~K}$, the relative error for the binary and ternary mixtures under study was $2.59 \times 10^{-3}$ and $2.42 \times 10^{-3}$ respectively.

Furthermore, we compared the temperature coefficient $d n / d T$ (at constant concentration)' and concentration coefficient $d n / d C$ (at constant temperature and wavelength) of refractive indices. For the binary mixtures $\left(n_{T H N}\right) n C_{12},\left(n_{T H N}\right) I B B$ and $\left(n_{I B B}\right) n C_{12}$, the average standard deviation of the temperature coefficient and the concentration coefficient of refractive index did not exceed $\left(6.753 \times 10^{-6}\right)$ [15], and $\left(2.491 \times 10^{-3}\right)$ [5] respectively.

Table 3.2: The estimated coefficients of the proposed correlation were obtained using a multi-input regression analysis and an analysis of variance ANOVA using MatLab®.

\begin{tabular}{ccccccc}
\hline \multicolumn{2}{c}{ Estimated Coefficients } & $\begin{array}{c}\text { Standard } \\
\text { Error }\end{array}$ & t Stat & Mean Sq & DF & F \\
\hline THN & 1.72187 & $8.393 \mathrm{E}-03$ & 205.1463 & 4.3878 & 1 & $4.2085 \mathrm{E}+04$ \\
$\mathrm{IBB}$ & 1.67452 & $8.394 \mathrm{E}-03$ & 199.4951 & 4.1494 & 1 & $3.9798 \mathrm{E}+04$ \\
$n \mathrm{C}_{\mathbf{1 2}}$ & 1.60631 & $8.397 \mathrm{E}-03$ & 191.3017 & 3.8155 & 1 & 36596.3245 \\
Temperature K & $-4.798 \mathrm{E}-04$ & $2.689 \mathrm{E}-05$ & -17.8470 & 0.0332 & 1 & 318.5158 \\
Wavelength $n m$ & $-8.257 \mathrm{E}-05$ & $2.826 \mathrm{E}-06$ & -29.2154 & 0.0890 & 1 & 853.5383 \\
\hline
\end{tabular}

Number of observations: 1980, Error 7.1525E-03, Error degrees of freedom: 1975 Mean Squared Error: 3.6215E-06 
Table 3.3: Standard deviations of the experimental results in this study and its corresponding found in literature.

\begin{tabular}{|c|c|c|c|c|c|}
\hline \multirow{2}{*}{ Compounds } & \multicolumn{4}{|c|}{ SD of $(d n / d T)^{a}$} & \multirow{2}{*}{ SD of $(d n / d c)^{b}$} \\
\hline & 657.2 & 632.9 & $\overline{589.3}$ & 436.1 & \\
\hline$n \mathrm{C}_{12}$ & $2.34 \mathrm{E}-06$ & $9.31 \mathrm{E}-07$ & $3.70 \mathrm{E}-05$ & $7.51 \mathrm{E}-07$ & - \\
\hline IBB & $2.02 \mathrm{E}-05$ & $9.96 \mathrm{E}-06$ & $2.86 \mathrm{E}-06$ & 4.55E-06 & - \\
\hline THN & $1.91 \mathrm{E}-05$ & $1.28 \mathrm{E}-05$ & $2.11 \mathrm{E}-05$ & $1.85 \mathrm{E}-07$ & - \\
\hline $\mathrm{IBB} / n \mathrm{C}_{12}$ & $4.61 \mathrm{E}-06$ & $6.67 \mathrm{E}-06$ & $5.07 \mathrm{E}-06$ & $2.84 \mathrm{E}-07$ & $2.52 \mathrm{E}-04$ \\
\hline $\mathrm{THN} / n \mathrm{C}_{12}$ & 4.44E-06 & $1.84 \mathrm{E}-06$ & $1.20 \mathrm{E}-05$ & $1.15 \mathrm{E}-05$ & $2.52 \mathrm{E}-04$ \\
\hline THN/IBB & $1.76 \mathrm{E}-05$ & $6.89 \mathrm{E}-06$ & $1.14 \mathrm{E}-05$ & $9.11 \mathrm{E}-06$ & $2.98 \mathrm{E}-04$ \\
\hline THN/IBB/ $n \mathrm{C}_{12}$ & - & - & - & - & $1.37 \mathrm{E}-03$ \\
\hline
\end{tabular}

${ }^{a}$ Standard deviation (SD) of the temperature coefficient of refractive index $(\mathrm{dn} / \mathrm{dT})$ at temperature range of (293.16 to $308.15 \mathrm{~K}$ ) compared with the literature [15].

${ }^{\mathrm{b}}$ Standard deviation (SD) of the concentration coefficient of refractive index (dn/dC) at 298.1k at wavelength of $657.2 \mathrm{~nm}$ compared with the literature [5] at same temperature and wavelength of $670 \mathrm{~nm}$.

\subsection{Theoretical Calculation of Refractive Index}

The experimental data obtained during the study was evaluated using different empirical and semi-empirical models called "mixing rule" equations. Several mixing equations have been proposed in the literature [15], some of these include the Lorentz-Lorenz, Gladstone-Dale, Arago-Biot, Eykman, Lichtenecker and Oster Eq.'s 3.5 to 3.10. These equations can only be applied if there are no chemical reactions in the mixture and if there are no changes in volume during mixing. If these conditions are met, these relations can be used to predict the refractive indices of multi-component mixtures based on the knowledge of some of the physical properties of the pure components used in the mixture such as molar mass and refractive index. The most widely used equations are listed below:

$$
\begin{array}{ll}
\text { Lorentz-Lorenz (L-L) } & {\left[\frac{\mathrm{n}^{2}-1}{\mathrm{n}^{2}+2}\right]=\sum_{\mathrm{i}=1}^{\mathrm{N}}\left[\frac{\mathrm{n}_{\mathrm{i}}^{2}-1}{\mathrm{n}_{\mathrm{i}}^{2}+2}\right] \varphi_{\mathrm{i}}} \\
\text { Goldstone-Dale (G-D) } & \mathrm{n}_{\mathrm{D}}-1=\sum_{\mathrm{i}=1}^{\mathrm{N}}\left[\mathrm{n}_{\mathrm{i}}-1\right] \varphi_{\mathrm{i}} \\
\text { Arango-Biot(A-B) } & \mathrm{n}_{\mathrm{D}}=\sum_{\mathrm{i}=1}^{\mathrm{N}}\left(\mathrm{n}_{\mathrm{i}}\right) \varphi_{\mathrm{i}} \\
\text { Eykman (E) } & \frac{n^{2}-1}{n+0.4}=\sum_{i=1}^{N}\left[\frac{n_{i}^{2}-1}{n_{i}+0.4}\right] \varphi_{i} \\
\text { Lichtenecker } & \ln \mathrm{n}=\sum_{\mathrm{i}=1}^{\mathrm{N}} \varphi_{\mathrm{i}} \ln \mathrm{n}_{\mathrm{i}}
\end{array}
$$


Oster $(\mathrm{O})$

$$
\frac{\left(n^{2}-1\right)\left(2 n^{2}+1\right)}{n^{2}}=\sum_{i=1}^{N} \frac{\left(n_{i}^{2}-1\right)\left(2 n_{i}^{2}+1\right)}{n_{i}^{2}} \varphi_{i}
$$

where $n$ is the refractive index of the mixture and $n_{i}$ is that of $i^{\text {th }}$ pure component. $\varphi_{i}$ is the volume fraction of the $i^{\text {th }}$ component, obtained by Eq. 3.4.

The present study used these mixing rules in order to predict the refractive indices of the ternary mixtures under study. These theoretical estimations were also used to verify and validate the experimental measurements and the results of the proposed empirical correlation. The estimated values of the refractive indices were found to be in good agreement with the experimental results, with the distinction of Arago-Biot (A-B) which revealed better agreement, with two decimal place order of magnitude, when compared to Oster O. The ternary mixture of $\left(n_{I B B}\right)_{n C_{12}} \mid T H N=0.5$ at wavelengths of 657.2 and $298.15 \mathrm{~K}$ was used as an example in Figure 3-5 which depicts the comparison between the proposed experimental values, those obtained by the proposed correlation, the values calculated using Eq. 3.3, and the theoretical values obtained using the mixing rule equations with the average relative error of $5.447 \times 10^{-4}, 3.001 \times 10^{-3}$, and $1.677 \times 10^{-2}$ respectively. The results reveal that the proposed data are sufficiently accurate.

(a)

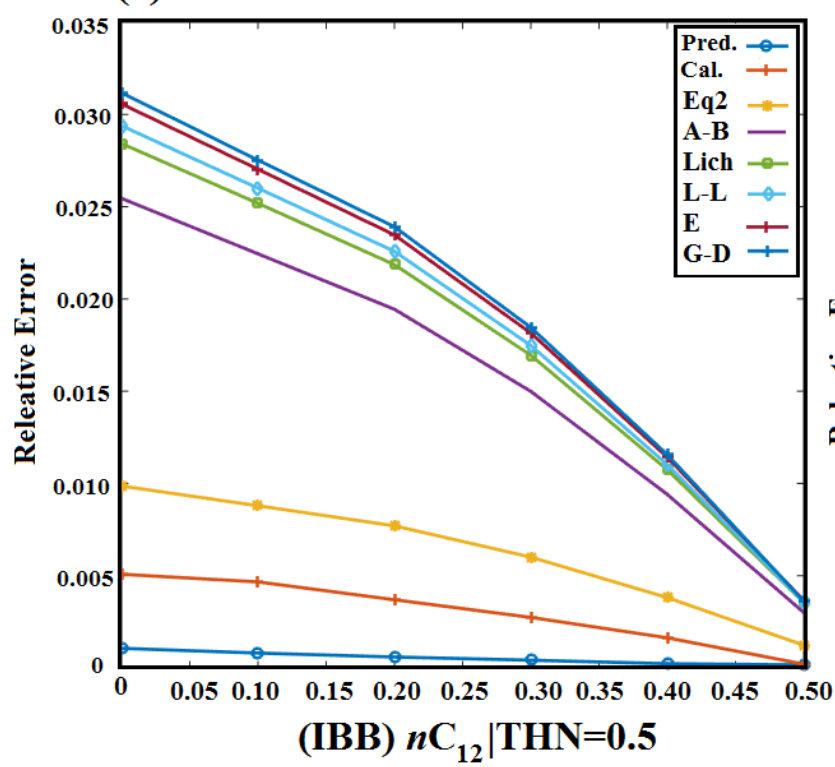

(b)

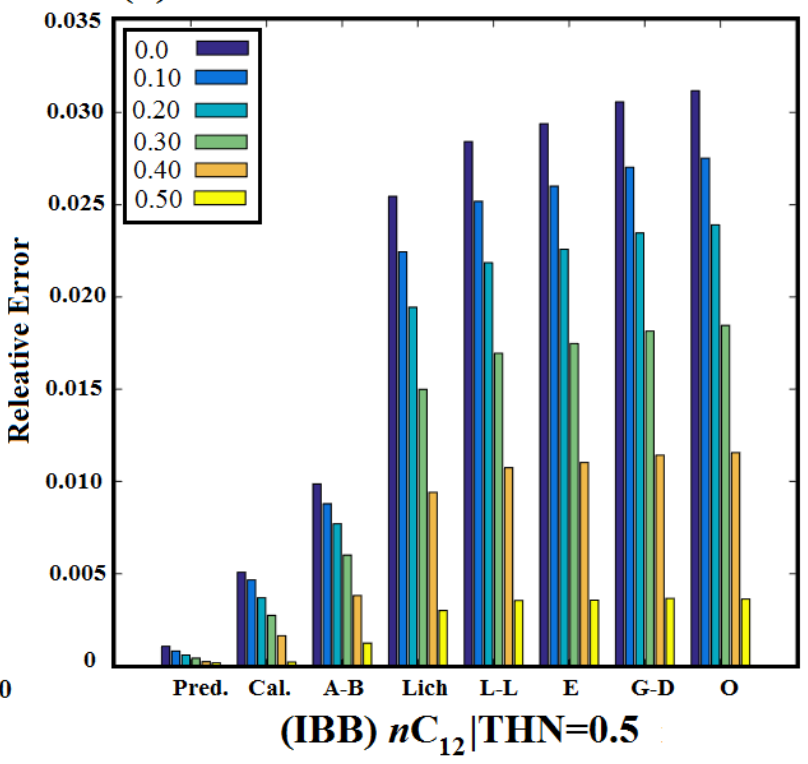

Figure 3-5: a- Relative error of the experimental refractive index values with the predicted, calculated and estimated values using mixing rules for various compositions of the $\left(\mathrm{n}_{\mathrm{IBB}}\right) n \mathrm{C}_{12} \mid \mathrm{THN}$ at $T=298.15 \mathrm{~K}$ at a wavelength $0 \mathrm{f57.2} \mathrm{nm}$. b- Relative error of the same mixture at different IBB concentration. 


\subsection{Conclusions}

Organized and highly accurate experimental measurements of the refractive indices were conducted of pure, binary and ternary mixtures consisting of the hydrocarbon substances Dodecane $n \mathrm{C}_{12}$, Isobutylbenzen $\mathrm{IBB}$, and Tetrtahydronaphtalenene $\mathrm{THN}$ with respect to concentration and the temperature derivative $(\partial \mathrm{n} / \partial \mathrm{T})$. A total of thirty-six mixtures were carefully prepared (36 ternaries +27 binaries) and investigated along with their pure substances. The refractive indices were measured for multiple temperatures and wavelengths using the Abbemat Refractometer. The results of our investigation of the temperature, concentration, and wavelength dependency of refractive indices suggest a linear dependence with regard to concentration and temperature and a non-linear dependence with regard to wavelength. These results were in agreement with the theories and experimental data found in the published literature. We also developed and tested new empirical correlations which can be used to predict the refractive index of ternary mixtures as a function of concentration, temperature and wavelength with an average relative accuracy of $1.55 \times 10^{-3}$. This level of accuracy is comparable to that of the best mixing rule by order of magnitude. The proposed correlation was validated using experimental data and estimated data using mixing rules.

The measured experimental refractive indices were compared with those estimated by applying several mixing rules: Lorentz-Lorenz (L-L), Gladstone-Dale (G-D), Arago-Biot (A-B), Eykman (E), Lichtenecker (Linch), and Oster (O). The results revealed good agreement with the experimental values with the distinction of A-B which revealed better agreement, with two decimal place order of magnitude ,when compared to O. Using a wide range of temperatures, concentrations and wavelength measurement conditions allows researchers to obtain more information, increasing the depth of scientific knowledge and advancing scientific research. 


\section{CHAPTER 4 MEASUREMENT OF OPTICAL CONTRAST FACTORS IN TERNARY HYDROCARBON MIXTURES}

This chapter is based on the following published paper:

Yahya M. Saghir M. Z. Measurement of Optical Contrast Factors in Ternary Hydrocarbon Mixtures. PHYS CHEM LIQ. DOI:10.1080/00319104.2015.1084879.

\subsection{Introduction}

Many liquids found in nature are generally combinations of pure elements. The behaviour of these combinations is more complex than the behaviour of pure fluids. Knowing the behaviour of multicomponent systems is thus very important to the field of science and technology.

In the presence of a concentration gradient, molecular diffusion tends to reduce the concentration variation in the mixture. This phenomenon is governed by Fickian law which states that the mass diffusion flux is $\vec{J}_{D}=-\rho D \vec{\nabla} c$. In the equation, $\vec{\nabla} c$ the concentration gradient, $\rho$ the density and D represents the mutual mass diffusion coefficient.

The presence of a temperature gradient in an isotropic liquid induces a diffusive mass flux $\overrightarrow{\mathrm{J}}_{\mathrm{T}}=-\rho \mathrm{D}_{\mathrm{T}} \mathrm{c}(1-\mathrm{c}) \vec{\nabla} \mathrm{T}$ where $\mathrm{D}_{\mathrm{T}}, 1-\mathrm{C}$ are the thermal diffusion coefficient and weight factor respectively. In stationary conditions, the concentration adjusts such that $\overrightarrow{\mathrm{J}}_{\mathrm{T}}$ is balanced with $\overrightarrow{\mathrm{J}}_{\mathrm{D}}$ and as a result we obtain the thermal diffusion given by the equation $\mathrm{S}_{\mathrm{T}}=$ $\frac{-\nabla c}{c(1-c) \nabla \mathrm{T}}$. This phenomenon was discovered by Ludwig (1856) and Soret (1879), and named the Soret effect. The Soret coefficient $\mathrm{S}_{\mathrm{T}}=\mathrm{D}_{\mathrm{T}} / \mathrm{D}$ measures the concentration difference that can be sustained by a specified temperature difference between any two points in a liquid [82-84].

Thermao-diffusion phenomenon plays a crucial role in nature and technology. It contributes to various fields, playing a significant role in such things as crude oil experiments and the distribution of crude oil components [30]. The Soret effect has been adapted for use in the separation of isotopes in liquid and gaseous mixtures [10], as well as biological systems [11].

For most optical techniques measuring the Soret coefficient requires detailed knowledge of the variation of the refractive index as a function of concentration and temperature gradient, known as contrast factors $(\partial \mathrm{n} / \partial \mathrm{C})$ and $(\partial \mathrm{n} / \partial \mathrm{T})[14,19-21,91]$.

Refractive index is a fundamental optical property of liquids and can be examined with the help of quantitative refined optical techniques. These techniques make use of some, typically 
coherent, light source passing through a transparent medium to characterize some of their optical properties. The variation of the contrast factors of different mixtures is usually very small, making it important to have accurate measurements of their refractive indices. The refractive index correlates with the composition, temperature, pressure, and light source wavelength of a particular mixture [1-9].

Researchers have developed various techniques in order to attempt to provide accurate results of contrast factors and the thermal diffusion coefficient for binary and multicomponent mixtures. These include optical beam deflection (OBD) [58,59], thermal diffusion forced Rayleigh scattering [22,24,25], thermal lensing [23], and optical digital interferometry (ODI) $[12,13,15]$.

The Abbemat Refractometer and Mach-Zehnder Interferometer (MZI; optical digital interferometry) techniques were adopted in this study. These optical measurement techniques are known as some of the most precise methods of measuring the contrast factors and diffusion coefficients in a transparent medium.

The MZI technique is unique in that it allows for the possibility of changing the temperature and concentration of the mixture during measurement without disturbing the experiment. It also allows for the observation of the entire cross-section of the working field. One of the limitations of this method is that the liquid being examined must be transparent to light.

The refractive index of transparent liquid mixtures can also be estimated using a suitable mixing rule equation. These mixing rules make use of the known physical properties of the pure components that form the multicomponent mixture in order to predict the refractive index of the mixture. Some notable mixing rules proposed in the literature include Lorentz-Lorenz (L-L), Gladstone-Dale (G-D), Arago-Biot (A-B), Eykman (E), Wiener (W), Heller (H), Newton (N), Lichtenecker (L) and Oster $(\mathrm{O})$. These mixing rule equations have been tested and validated by several researchers $[17,85,86,92,93]$.

In the present study we attempt to provide experimental measurements of the refractive indices of two ternary systems consisting of three hydrocarbon components of two-ring component 1,2,3,4-Tetrahydronaphtalene (THN), one-ring component Isobutylbenzene (IBB), and an alkane $n$-Dodecane $\left(\mathrm{C}_{12}\right)$. Knowledge of THN-IBB- $\mathrm{C}_{12}$ mixtures is of great interest for Differential Scanning Calorimetry (DSC) and Influence of Vibrations on diffusion in Liquid 
(IVIDIL) experiments currently taking place (on ground and in space) onboard the International Space Station (ISS) [31].

Although the optical properties of the pure substances and some of the binary mixtures can be found in the literature, there is a limited amount of information regarding ternary systems $[5,15,93,94]$.

The refractive indices of two different ternary systems of THN, IBB and $n C_{12}$ were measured using the following concentration targets: molar fraction of (0.501-0.110-0.389) and (0.441-0.217-0.342). In order to calculate the temperature and concentration derivative ( $\partial n$ / $\partial T)_{C_{o} \lambda}$ and $(\partial n / \partial C)_{T_{o} \lambda}$ of the refractive index we prepared and investigated eleven mixtures for each of the mentioned compositions: $\left(n_{T H N}\right)_{n C_{12}}\left|I B B, \quad\left(n_{n C_{12}}\right)_{I B B}\right| T H N$ and $\left(n_{T H N}\right)_{I B B} \mid n C_{12}$. Measurements were conducted on a wide range of different temperatures and wavelengths. These measurements were also taken for each of the pure components.

In the following section we present the sample preparation methods used in this study. In section three we present a concise summary of the techniques used to measure the refractive indices. In section four we present the results of our refractive index calculations using experimental methods as well as predictive mixing rules and provide a discussion of our findings.

\subsection{Mixture Preparation}

The three hydrocarbon squalane 1,2,3,4-Tetrahydronaphtalene (THN; chemical formula $\mathrm{C}_{10} \mathrm{H}_{12}$ ), Isobutylbenzene (IBB; chemical formula $\left.\mathrm{C}_{4} \mathrm{H}_{8}\right)$ and $n$-Dodecane $\left(n \mathrm{C}_{12}\right.$; alkane hydrocarbon; chemical formula $\mathrm{C}_{12} \mathrm{H}_{26}$ ) were purchased from Acros Organics®. The stated purity of the liquids was between 98 and $99 \%$ and the liquids were used without any further purification.

Two ternary compositions with molar fraction of (0.501-0.110-0.389) and (0.441-0.2170.342 ) corresponding to (THN-IBB- $n \mathrm{C}_{12}$ ) were selected to be examined in this study. In order to obtain the temperature and concentration derivative of the refractive index (contrast factors) for the desired compositions we prepared 33 mixture samples for each composition. The desired mixtures were prepared using pre-prescribed mass fraction with a sampling rate of $10 \%$ for each component, adding known masses of the pure liquid in leak proof $5 \mathrm{ml}$ glass vials. In order to minimize component evaporation and any errors in the composition, the sample mixtures were 
prepared by adding the least volatile component (i.e., the alkane $n \mathrm{C}_{12}$ with higher molecular weight) followed by the corresponding amount of the second substance with a component with higher volatility and then a third substance with the component with the highest volatility. The prescribed mass fraction of each component was measured using a Denver Instrument ${ }^{\circledR}$ SI-234 balanced with a precision of 4 decimal places $\pm 1 \times 10^{-3} \mathrm{~g}$, and all ingredients were added using a pipette dropper. 66 mixtures of different concentrations were prepared at an accuracy of $\pm 1 \times 10^{-3}$ $\mathrm{g} / 5 \mathrm{~g}$ based on the average sampling errors and instrument sensitivity. The molar fractions were then calculated for all compositions based on the prepared mass fraction values and the constant molar mass of the pure components.

The mixtures were prepared in a fume hood lab bench at a room temperature of $296 \mathrm{~K}$ with \pm $2 \mathrm{~K}$ stability. In order to ensure the accuracy of our results, the mixtures were prepared and examined three times on different days. The mixtures were used immediately following preparation in order to minimize evaporation and reduce measurement error.

\subsection{Experimental Methods}

Laser-based techniques were used to measure changes in the refractive index of ternary mixtures $\mathrm{THN}, \mathrm{IBB}$ and $n \mathrm{C}_{12}$, for different compositions. The concentration of the mixtures under investigation was determined by varying the mass fraction of each component. Variations of the refractive index of a liquid depend on its concentration, temperature and the wavelength of the incident light (pressure effects are here ignored). Thus, for an isobaric condition, change in the refractive index of liquids is determined as a function of concentration, temperature and wavelength using refractomery and interferometry techniques.

\subsubsection{Abbemat Refractometer}

The refractive indices of three hydrocarbon components and two ternary mixture compositions were measured using an automatic Anton Paar® WR Refractometer. This refractometer works with multiple visible wavelengths between (436.1 and 657.2) $\mathrm{nm}$. The working temperature range of this instrument is from (283 to 343$) \mathrm{K}$, the instrument resolution is $\pm 1 \times 10^{-6}$, and the accuracy is $\pm 4 \times 10^{-5}$. The average standard deviation of the mean "standard uncertainty" of the instrument, expressed by $u_{i}=\frac{a}{\sqrt{3}}$, (where a represent the semi-range between the upper and lower limits) were estimated to be $4.7 \times 10^{-5}$. 
When measuring the refractive index, this device uses reflected light instead of the transmitted light used in ODI. The sample is placed in a sample mold on top of the measuring YAG prism (Yttrium-Aluminum-Garnet) that irradiates from different angles via an LED light. At the interface between sample and prism, the incident beam is either refracted into the sample or reflected back into the prism. The reflected beam is detected by a sensor array. The critical angle for total reflection is then calculated and used to determine the refractive index of the sample based on Snell's law [88].

The temperature of the prism was controlled by a Peltier thermostat with temperature sensors at an accuracy of $\pm 0.03 \mathrm{~K}$ and stability of $\pm 0.002 \mathrm{~K}$ in the working temperature range. A high-power LED was used as the light source passed through an interference filter yielding different wavelengths with a wavelength error of $\pm 0.1 \mathrm{~nm}$. The instrument was calibrated before each series of measurements using distilled water. The calibrations were conducted in accordance with a specifications table supplied by the refractometer manufacturer at atmospheric pressure. The prism was cleaned with Acetone after each calibration and sample measurement and dried with lab wipes.

While the instruments were being calibrated, the mixtures were placed on a magnetic stirrer for several minutes to ensure homogeneity. To perform the desired measurements, the temperature of the refractometer was set at $293 \mathrm{~K}$ and a few drops of the sample were placed on the main prism surface of the Abbemat Refractometer. The digital reading of the refractive index was then recorded. We also recorded the refractive indices of five different wavelengths (657.2, $632.9,589.2,513.9$ and 436.1) $\mathrm{nm}$ for each temperature. The temperature was then increased to $318 \mathrm{~K}$ with $5 \mathrm{~K}$ interval and the refractive indices were recorded for the aforementioned range of temperatures and wavelengths. This procedure was first used to measure the refractive indices of the three pure liquids $\mathrm{THN}, \mathrm{IBB}$, and $n \mathrm{C}_{12}$. The same procedure was then repeated to measure the refractive indices of all 66 investigated THN-IBB- $n \mathrm{C}_{12}$ mixtures.

The primary reason for measuring the pure components was to ascertain the accuracy of the results. These measurements were then used to estimate the refractive indices using the mixing rule equations discussed in section 4.6. All measurements were repeated three times within the same condition at the same atmospheric pressure $\mathrm{P}=0.1 \mathrm{MPa}$ in order to increase measurement accuracy. The average standard uncertainty of the repeated measurements $u_{r}=\frac{S D}{\sqrt{n}}(S D$ 
represents the standard deviation for the number of measurements $n$ ), calculated based on the mean values of the repeatability of refractive index measurements was $2.5 \times 10^{-4}$.

\subsubsection{Mach-Zehnder Interferometer}

The Mach-Zehnder Interferometer (MZI) is an experimental optical arrangement capturing light interference phenomenon caused by a relative phase shift variation between two collimated coherent light beams. This technique was discovered by Ludwig Mach and Ludwig Zehnder at the end of the eighteenth century.

This technique requires a collimated coherent light source in combination with two beam splitters with $50 \%$ reflectivity and two high reflection mirrors aligned at specific angles, generally 45 degrees (square or rectangular geometry). An image recording system is also utilized in order to record the experimental results. For this experiment we chose a 35 degree angle parallelogram shaped optical arrangement [96], allowing us to have a larger area of interference light covering a wide area of the working cell. A schematic drawing of the setup used in this study is shown in Figure 4-1.

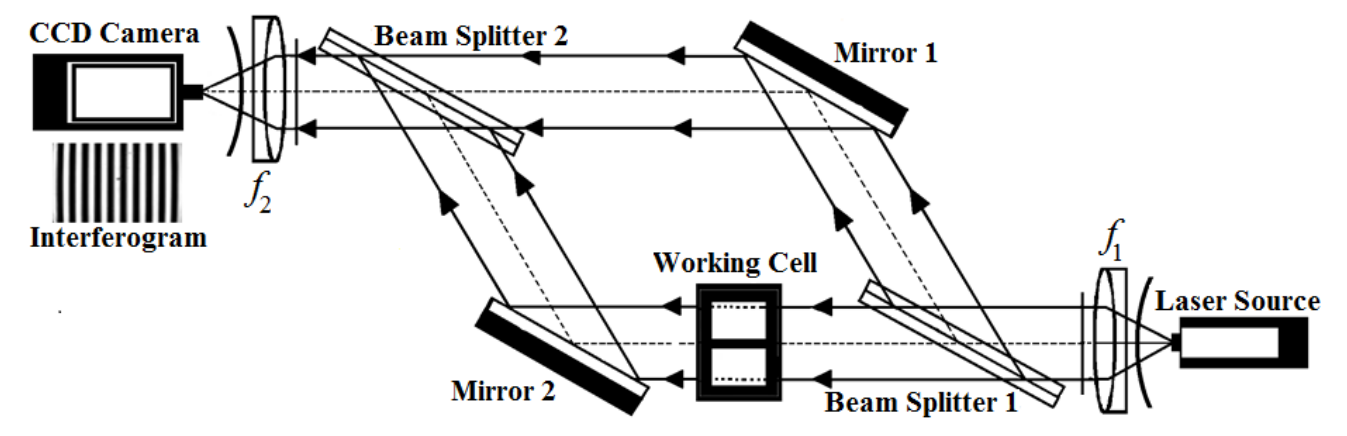

Figure 4-1: Schematic drawing of the Mach-Zehnder Interferometer setup used in this study.

The light source used for this portion of the experiment was a single $15 \mathrm{~mW}$ coherent visible red light at a wavelength of $632.9 \mathrm{~nm}$, emitted from a He-Ne laser (CVI Melles Griot@ 25-LHP151-249). The visibility of the light source provides a significant advantage for the optical apparatus alignment. Since the diameter of the laser beam is very small (in the range of 1-2 $\mathrm{mm}$ ), a spatial filter with a 10x microscope objective lens was placed in front of the laser source to expand the light beam and reduce light intensity in order to avoid sensor damage or saturation of the CCD camera. Two pinholes were positioned in the light path in order to detract the diffracted light and eliminate undesired light on the working cell. Lastly, we used a Plano-Convex lens 
with a focal length of $f_{1}=3.5 \mathrm{~cm}$ to expand and collimate the laser into parallel rays. The whole set up was mounted on a 'RS2000 series' tuned vibration damping optical table (Newport corporation, dimension $1.2 \times 2.4 \times 0.203 \mathrm{~m}$, model: M-RS2000-48-8).

The first beam splitter (BS1) was placed in the focal plane of the collimated rays where the incident beam is split into two identical beams of equal intensity. The transmitted portion of the laser beam is directed to the first mirror (M1) where it is deflected back to the second beam splitter (BS2). The reflected portion of the laser beam is then deflected by a metallic mirror (M2) and directed onto the working cell. The propagated portion of the laser beam from the working cell is passed through the second beam splitter (BS2). The two beams travel through different paths (BS1-M1-BS2 and BS1-M2-working cell-BS2) for an equal distance and are then combined by a second beam splitter (BS2) to interfere. The transmitted interfering light beam from the second beam splitter (BS2) is then focused back using another convex lens $\left(f_{2}=\right.$ $2.5 \mathrm{~cm}$ ) and then eventually propagated to the image recording system. A slight difference in the optical path and/or difference in refractive index create a phase change.

The image recording system consists of a charge-coupled device CCD camera (JAI progressive scan, model: CV-M4 CL+ monochrome digital megapixel) with a resolution of $1280 \times 1024$ pixels and an approximate image scale of $60 \mathrm{pixel} / \mathrm{mm}$. The recording system is connected to a laboratory computer which then stores all of captured images known as 'Interferogram'.

The conventional block cell holder used in the literature [15] to measure the contrast factors Figure 4-2A were developed and used in this study. A diagram of the developed cell holder can be seen in Figure 4-2B.

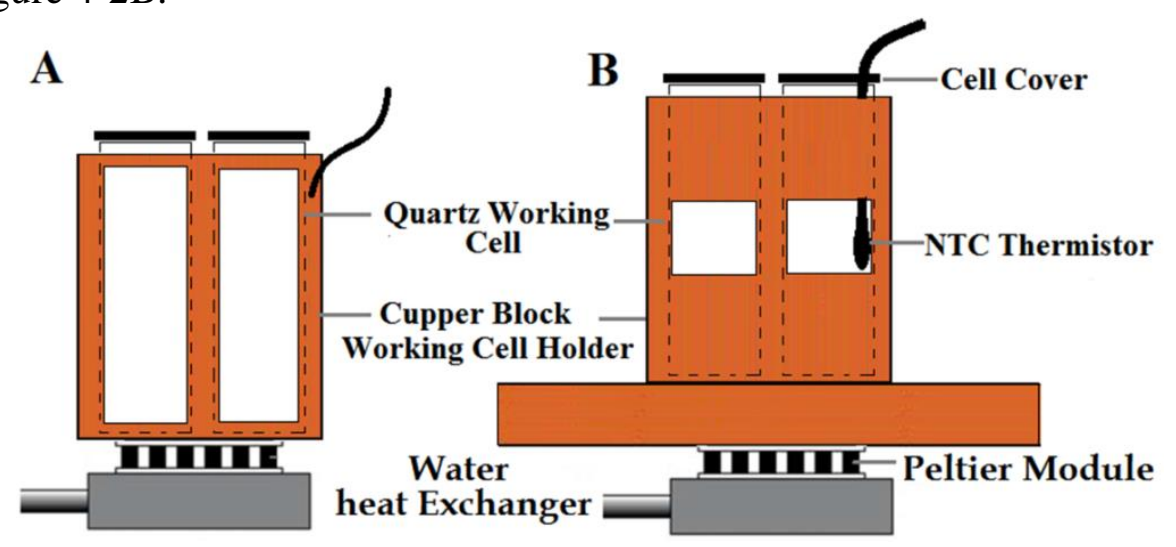

Figure 4-2: Schematic drawing of the cell holder used in the Mach-Zehnder interferometer: A- The classic cell holder used in the literature, B- the cell holder developed by the authors and used in this study. 
The outer dimensions of the cell holder are $30 \times 30 \times 40 \mathrm{~mm}$. When compared with the conventional cell holder, the newly developed cell holder achieved proper and accurate temperature distribution patterns along the examined mixtures see Figure 4-3. The cell holder was placed in the BS1-M2-BS2 path and heated by a Peltier module regulated by a proportionalintegral-derivative (PID) temperature controller system. The PID controller was used to generate uniform temperature gradient intervals and/or to maintain the desired temperature of the working cell. The other side of the Peltier module was coupled with a thermostat controlled water heat exchanger used as a heat sink due to the relatively large temperature differences and long experimental time. The pre-experimental stages were conducted on the pure elements THN, IBB and $n \mathrm{C}_{12}$ to obtain the optimal values for the PID parameters and suitable Peltier output heating power.
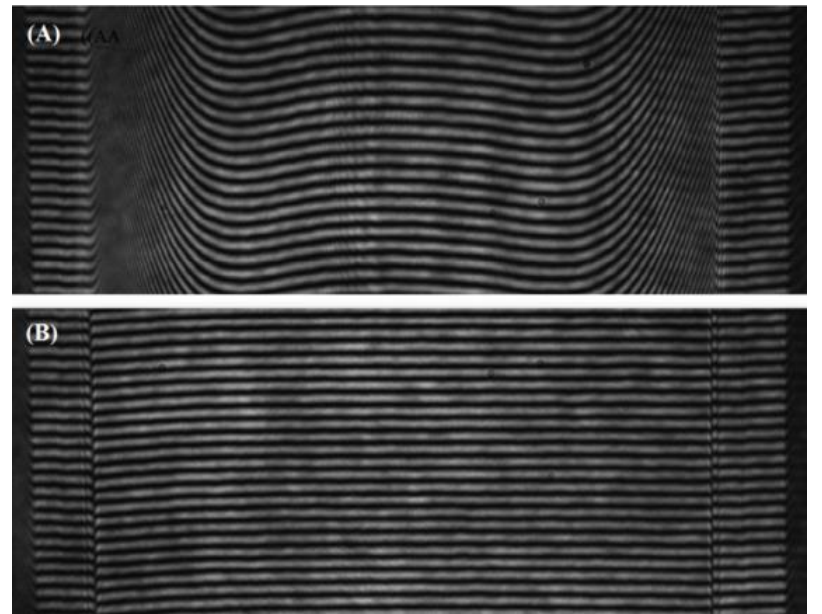

Figure 4-3: Interferogram sample show the fringes quality and the temperature distribution: A- Fingers obtained using the classic cell holder found in the literature, B- Fingers obtained using the developed cell holder showing a proper and accurate temperature distribution patterns along the examined mixtures.

In order to measure the temperature derivative of the refractive index $(\partial n / \partial T)_{\mathrm{C}_{0}, \lambda}$, a $10 \times 10 \times 40 \mathrm{~mm}$ quartz cell (Helma® QS $10 \mathrm{~mm}$ optical path) was filled with the desired liquid mixture and inserted in one of the cell holder groves. A calibrated NTC thermistor was immersed into the mixture of the covered quartz cell in order to measure the mixtures' temperature and provide a feedback temperature for the PID controller.

The maximum output power of the PID controller was set to $30 \%$ for all of the experimental measurements in order to achieve the appropriate heat gradient and accurate temperature distribution over the mixture. Based on the pre-experimental measurements, this was the optimal 
value. Although the temperature interval was set in the range of (291to 308) K, only the temperature interval range of (295to 303) $\mathrm{K}$ was considered in the final calculations. This temperature range was chosen to improve the accuracy of the experimental results. The heating process began at the same time as the CCD camera image recording at a one second interval in order to relate the phase change in the examined mixtures to the temperature change with the time $d T / d t$.

In order to measure the concentration derivative of the refractive index $(\partial n / \partial C)_{T_{o} \lambda}$, two quartz cells (Helma QS $10 \mathrm{~mm}$ optical path) were filled with a liquid mixture of the same composition and inserted into the copper cell holder. One of the two quartz cells remained unchanged throughout the entire experiment and was used as a reference cell in order to minimize error due to external vibration and other background noises. A pre-calculated liquid mixture of a different composition was injected into the second quartz cell using a syringe pump (Fisher Scientific ${ }^{\circledR}$ catalogue no. 14831200) with a constant injection rate of $0.5 \mathrm{ml}$ per hour. Images were taken at each second time step using the CCD camera. The copper block cell holder was placed on top of a heater/magnetic stirrer for instantaneous mixing at each desired temperature. The injection rate and the total volume of the added solution were manually imputed into the syringe pump. The Interferogram patterns of both reference and target images were recorded using the CCD camera. We then calculated the temperature and concentration derivative $(\partial n / \partial T)_{C_{o} \lambda}$ and $(\partial n / \partial C)_{T_{o} \lambda}$ of the refractive indices of the THN, IBB and $n \mathrm{C}_{12}$ directly from the slope of the unwrap phase discussed in the next section.

\subsection{Data Analysis and Image Processing}

The Interferogram images recorded by the CCD camera, known as fringe patterns, contain all the phase information of interest. Image processing was then used to analyze the fringe patterns in order to determine the refractive index profiles of the mixtures. According to the literature, two common methods are suggested for the analysis of Interferogram images to determine the phase change from the fringe: Phase Shifting Interferometry (PSI) and Fast Fourier Transform (FFT) [37,70,97].

The PSI method requires a minimum of three phase shifted interferogram to obtain the phase change and a simple algorithm to extract the phase information. The FFT method is widely used in the literature and requires only one interference fringe pattern to extract the phase information. It can also automatically determine the phase mapping without needing to locate the fringe 
centers or assign fringe orders. It is for these reasons that the FFT technique was adopted in this study.

Consider the intensity distribution of the interference pattern below:

$$
g(x, y)=a(x, y)+b(x, y) \cos \varphi(x, y)
$$

$a(x, y)$ and $b(x, y)$ represent the undesired illumination produced by background light and reflected light from surrounding objects, and $\varphi(x, y)$ represents the phase that contains all of the required information. The cosine represents the periodic function. The $\cos \varphi \operatorname{can}$ be represented by $\frac{1}{2}\left[e^{i \varphi(x, y)}+e^{-i \varphi(x, y)}\right]$. Substitute $\cos \varphi$ in Eq. 4.1 and the intensity function can be written as:

$$
\begin{aligned}
& g(x, y)=a(x, y)+b(x, y) \frac{1}{2}\left[e^{i \varphi(x, y)}+e^{-i \varphi(x, y)}\right] \\
& g(x, y)=a(x, y)+\frac{1}{2} b(x, y) e^{i \varphi(x, y)}+\frac{1}{2} b(x, y) e^{-i \varphi(x, y)}
\end{aligned}
$$

where i represents the imaginary unit $\mathrm{i}=\sqrt{-1}$. Applying a two-dimensional discrete FFT algorithm on Eq. 4.4, the intensity function in frequency domain yields:

$$
G(f, v)=A(f, y)+C(f, y)+C^{*}(f, y)
$$

where $A(f, y)$ is the transform of $a(x, y)$, and $C(f, y)$ and $C^{*}(f, y)$ are the positive and negative frequency spectra of the modulated carrier fringes and * represents the complex conjugation. By applying a band-pass filter on the intensity signal of Eq. 4.4 in the frequency domain, we can eliminate the effect of background illumination $A(u, v)$, and one of the terms $C(f, y)$ or $C^{*}(f, y)$. If we eliminate $C^{*}(f, y)$ and apply the inverse Fourier transform on the remaining term $C(u, v)$ we obtain:

$$
c(x, y)=R_{e}[c(x, y)]+i^{*} I_{m}[c(x, y)]
$$

The interference phase can then be calculated using the following equation:

$$
\varphi(x, y)=\arctan \left(\frac{I_{m}[c(x, y)]}{R_{e}[c(x, y)]}\right)
$$


where $R_{e}[c(x, y)]$ and $I_{m}[c(x, y)]$ represent the real and imaginary terms of $c(x, y)$.

Finally, in order to obtain continuity from the phase values that are produced by Eq. 4.6, a trivial image unwrapping procedure is needed. This can be done by adding or subtracting $2 \pi$ from each wrapped phase value.

On the other hand, the phase difference between all of the experimentally recorded images and the first reference image can be expressed with the following equation:

$$
\Delta \varphi\left(x, y, t_{i}\right)=\varphi\left(x, y, t_{i}\right)-\varphi\left(x, y, t_{o}\right)
$$

where $\varphi\left(x, y, t_{i}\right)$ is the ith image phase and $\varphi\left(x, y, t_{o}\right)$ is the reference image phase, taking in consideration a homogenous distribution of temperature and concentration inside the liquid. Since the refractive index varies with the temperature and concentration of the liquid mixture, we can express the phase change in the mixture due to changes in refractive index using:

$$
\Delta \varphi\left(x, y, t_{i}\right) \propto n\left(x, y, t_{i}\right)-n_{o}\left(x, y, t_{o}\right)
$$

where $n\left(x, y, t_{i}\right)$ is the refractive index at point ith with respect to initial value of $n_{o}\left(x, y, t_{o}\right)$. If $\mathrm{L}$ is the optical path length inside the homogenous mixture and $\lambda$ is the wavelength of the light source, then:

$$
\begin{gathered}
\Delta \varphi\left(x, y, t_{i}\right)=\frac{2 \pi L}{\lambda} \Delta n\left(x, y, t_{i}\right) \\
\Delta n\left(x, y, t_{i}\right)=\frac{\lambda}{2 \pi L} \Delta \varphi\left(x, y, t_{i}\right)
\end{gathered}
$$

In isobaric condition, the change of refractive index $\Delta n\left(x, y, t_{i}\right)$ is related to the change of temperature, concentration or both.

$$
\Delta n(x, y, t)=\left(\frac{\partial n}{\partial C}\right)_{T_{o} C_{o} \lambda} \Delta C(x, y, t)+\left(\frac{\partial n}{\partial T}\right)_{T_{o} C_{o} \lambda} \Delta T(x, y, t)
$$

where, $\Delta C(x, y, t)$ and $\Delta T(x, y, t)$ are the variation of concentration and temperature of point $(x, y)$ at time $t$. 


\subsection{Results and Discussion}

The temperature and concentration derivative $\left((\partial n / \partial T)_{C_{o} \lambda}\right.$ and $\left.(\partial n / \partial C)_{T_{o} \lambda}\right)$ of refractive indices at constant concentration and temperature were simultaneously measured under the same conditions using both the Abbemat Refractometer and MZI techniques. The experiments were repeated three times for each technique and the average was used for calculations. The experimental measurements were conducted using a temperature range of (293 to 318) K with multiple wavelengths between (657.2 and 436.1) $\mathrm{nm}$ and different concentration for each of the investigated systems. It is notable that the MZI measurements were conducted using a single wavelength of $632.9 \mathrm{~nm}$. The target mixtures of the two selected systems are (0.501-0.110-0.389) and (0.441-0.217-0.342) corresponding to (THN-IBB- $n \mathrm{C}_{12}$ ). Measurements of all pure components were also conducted in order to identify the compositional accuracy of the systems in question. The samples were examined at isobaric condition.

Analysis of the data reveals a linear relationship between refractive indices and concentrations as well as temperatures. The results revealed a non-linear relationship as a function of wavelength. This behaviour was observed in the mixtures as well as the pure liquids. Only some of the data was selected to illustrate our findings in the discussion section. The remainder of the data is attached to the appendix 4.1-4.3.

\subsubsection{Abbemat Refractometer}

The experimental values for the refractive indices of the pure liquids THN, IBB, and $n \mathrm{C}_{12}$ obtained using the Abbemat Refractometer as well as the temperature derivative of the refractive index $(\partial n / \partial T)_{C_{o} \lambda}$ are gathered in Table $\mathrm{S} 1$ available in the supplementary material. This tables also provides a comparison of our results with some of the wavelengths examined in the literature $[15,94,95]$, and reveals substantial agreement with an average standard deviation of $2.8 \times 10^{-4}, 2.5 \times 10^{-4}$ and $5.2 \times 10^{-6}$ respectively. Our investigation of the temperature derivative of the refractive index $(\partial n / \partial T)_{C_{o} \lambda}$ revealed a linear decrease in refractive indices with increases in temperature. This behaviour was observed in the mixtures as well as the pure substances. On the other hand, the results revealed a non-linear relationship between refractive index and wavelength variation. This behaviour was observed in the mixtures as well as the pure substances. Figure S1(a-b) in the supplementary material provides a graphical illustration of the relationships between refractive indices with temperature and wavelength variations in pure 
substances. Our investigation of the ternary mixtures revealed the same behaviour as the pure substances; whereas the concentration derivative $(\partial n / \partial C)_{T_{0} \lambda}$ and temperature derivative index $(\partial n / \partial T)_{C_{o} \lambda}$ of the refractive index revealed a linear relationship with concentration and temperature for all mixtures.

For verification purposes, we calculated the average refractive indices of $\left(n_{T H N}\right)_{n C_{12}} \mid I B B$, $\left(n_{n C_{12}}\right)_{I B B} \mid T H N$ and $\left(n_{T H N}\right)_{I B B} \mid n C_{12}$ for the two target compositions at wavelengths of (657.2, $632.9,589.2,513.9$, and 436.1$) \mathrm{nm}$ at six different temperatures of (293 to 318) K. This was done using the refractive indices of the pure components and equation 4.12. The results can be seen in in the supplementary material Tables $\mathrm{S} 2$ and $\mathrm{S} 3$.

$$
\Delta n=n-\sum_{i=1}^{N} x_{i} n_{i}
$$

In this equation, $n$ is the refractive index of the mixture, $n_{i}$ is the refractive of the pure component $i$ and $x_{i}$ is the mole fraction of component $i$ in the mixture. $N$ is the number of components in the mixture [89-93].

The last two columns in these tables represent the standard deviation $(\sigma)$ and the relative of errors (RE) calculated using the following expressions:

$$
\begin{aligned}
\delta & =\sqrt{\frac{\sum_{i=1}^{N}\left(n_{\text {exp }}-n_{c a l}\right)^{2}}{N}} \\
R E & =\frac{\left|n_{\text {cal }}-n_{\text {exp }}\right|}{n_{\text {exp }}}
\end{aligned}
$$

where $n_{\text {exp }}, n_{\text {cal }}$ are the experimental and calculated values of the refractive index, and $N$ is the number of experimental data points. Figure 4-4 presents a clearer image of the linear and nonlinear relationships of the refractive indices with temperature and wavelength for the two target mixtures.

Our investigation of the concentration derivative of the refractive index $(\partial n / \partial C)_{T_{o} \lambda}$ revealed a linear relationship between refractive indices and concentration for all mixtures investigated. All mixtures were prepared based on the mass fraction of the components and the molar fractions were then calculated from the mass fraction. In order to provide an example of this relationship, the concentration dependence of the refractive index 
$\left(n_{T H N}\right)_{n C_{12}} \mid I B B=0.1$ and 0.2 for the two investigated systems at different wavelengths is presented in Table 4.1(a-b) and illustrated in Figure 4-5.

Table 4.1: The concentration dependence of the refractive index of using the Abbemat refractometer at a temperature $298.15 \mathrm{~K}$ for different wavelengths. a- for the $(0.501-0.110-0.389)$ system and $\mathrm{b}$ - for the (0.4410.217-0.342) system at atmospheric pressure $P=0.1 \mathrm{MPa}^{*}$.

\begin{tabular}{|c|c|c|c|c|c|c|}
\hline THN & IBB & 657.2 & 632.9 & 589.2 & 513.9 & 436.1 \\
\hline 0.451 & 0.111 & 1.46306 & 1.46410 & 1.46644 & 1.47112 & 1.48032 \\
\hline 0.461 & 0.111 & 1.46434 & 1.46511 & 1.46753 & 1.47251 & 1.48155 \\
\hline 0.471 & 0.111 & 1.46546 & 1.46630 & 1.46863 & 1.47378 & 1.48288 \\
\hline 0.481 & 0.110 & 1.46648 & 1.46755 & 1.46970 & 1.47498 & 1.48407 \\
\hline 0.491 & 0.110 & 1.46771 & 1.46871 & 1.47097 & 1.47605 & 1.48536 \\
\hline 0.501 & 0.110 & 1.46882 & 1.46998 & 1.47197 & 1.47721 & 1.48662 \\
\hline 0.511 & 0.109 & 1.47008 & 1.47113 & 1.47330 & 1.47857 & 1.48800 \\
\hline 0.521 & 0.109 & 1.47138 & 1.47219 & 1.47457 & 1.47986 & 1.48938 \\
\hline 0.531 & 0.109 & 1.47240 & 1.47340 & 1.47568 & 1.48101 & 1.49063 \\
\hline 0.540 & 0.109 & 1.47366 & 1.47476 & 1.47694 & 1.48233 & 1.49192 \\
\hline 0.550 & 0.108 & 1.47459 & 1.47586 & 1.47812 & 1.48350 & 1.49321 \\
\hline \multicolumn{2}{|c|}{$(\mathrm{dn} / \mathrm{dC}) \mathrm{THN} \mid \mathrm{IBB} \mathrm{B}^{\mathrm{a}}$} & $0.117577^{b}$ & 0.119874 & 0.11882 & 0.124198 & 0.130913 \\
\hline
\end{tabular}

Table 4.1b

\begin{tabular}{ccccccc}
\hline THN & IBB & 657.2 & 632.9 & 589.2 & 513.9 & 436.1 \\
\hline 0.391 & 0.220 & 1.46108 & 1.46201 & 1.46410 & 1.46907 & 1.47794 \\
0.401 & 0.219 & 1.46331 & 1.46428 & 1.46639 & 1.47144 & 1.48047 \\
0.411 & 0.219 & 1.46556 & 1.46653 & 1.46867 & 1.47381 & 1.48299 \\
0.421 & 0.218 & 1.46784 & 1.46883 & 1.47102 & 1.47623 & 1.48557 \\
0.431 & 0.218 & 1.47013 & 1.47112 & 1.47334 & 1.47865 & 1.48811 \\
0.441 & 0.217 & 1.47242 & 1.47344 & 1.47570 & 1.48107 & 1.49070 \\
0.451 & 0.217 & 1.47479 & 1.47583 & 1.47812 & 1.48357 & 1.49336 \\
0.461 & 0.216 & 1.47701 & 1.47852 & 1.48036 & 1.48586 & 1.49575 \\
0.470 & 0.215 & 1.47950 & 1.48057 & 1.48292 & 1.48854 & 1.49865 \\
0.480 & 0.215 & 1.48203 & 1.48310 & 1.48550 & 1.49120 & 1.50147 \\
0.490 & 0.214 & 1.48433 & 1.48543 & 1.48785 & 1.49363 & 1.50406 \\
\hline (dn/dC)THN $\mid$ IBB $^{\text {ac }}$ & 0.117037 & 0.118257 & 0.11951 & 0.123555 & 0.131318 \\
\hline
\end{tabular}

* The reported average expanded uncertainty $U=1.197 \times 10^{-3}$ was calculated based on the coverage factor $k=$ 2, providing a level of confidence of approximately $95 \%$. ${ }^{a}$ Concentration coefficients of refractive index obtained by Abbemat Refractometer. ${ }^{b}$ Current study against the literature [15] with average standard deviation of $1.9 \times 10^{-3}$. ${ }^{c}$ Current study against the literature [94] with average standard deviation of $1.6 \times 10^{-3}$. 
We can see that as the THN molar fraction increased from 0.451 to 0.550 and 0.391 to 0.490 , the corresponding molar fraction of $n \mathrm{C}_{12}$ decreased accordingly. The IBB molar fraction did not change very much (0.111 to 0.108 and 0.220 to 0.214 ). At a wavelength of $657.2 \mathrm{~nm}$ and 298.15 $\mathrm{K}$, the refractive index increased by more than one percent from 1.46306 to 1.47459 and more than two percent from 1.46108 to 1.48433 respectively.

The contrast factors of two THN-IBB- $n \mathrm{C}_{12}$ ternary systems were calculated using the refractive indices at different temperatures and concentrations for five wavelengths (657.2 to $436.1 \mathrm{~nm})$.
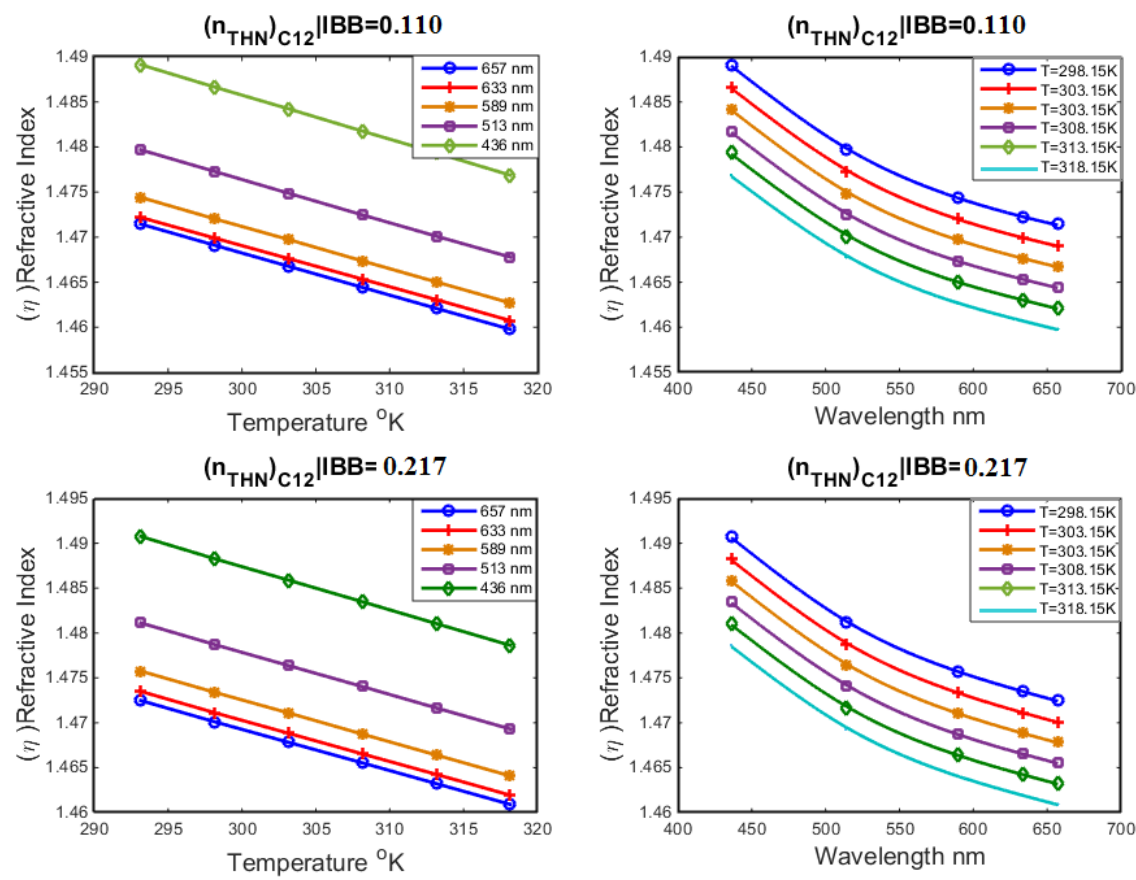

Figure 4-4: The refractive index of the two investigated systems: $(0.501-0.110-$ $0.389)$ and (0.441-0.217-0.342) corresponding to THN-IBB- $n$ C12 molar fraction.
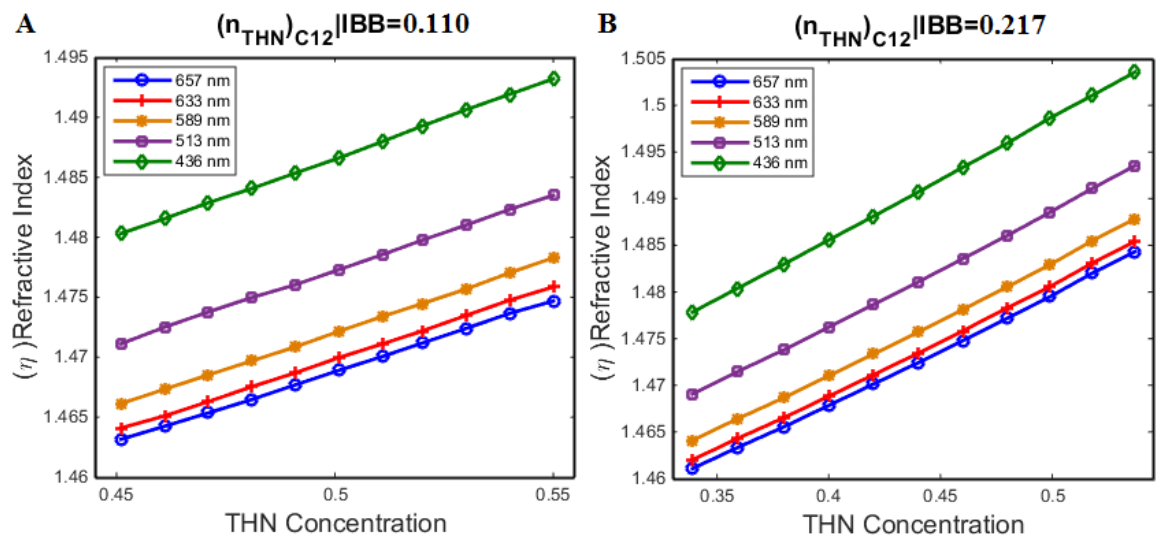

Figure 4-5: Concentration derivative of refractive indices for various compassions of $\left(\mathrm{n}_{\mathrm{THN}}\right) n \mathrm{C}_{12} \mid \mathrm{IBB}$ at a single temperature of $298 \mathrm{~K}$ with respect to the two investigated systems: $A-(0.501-0.110-0.389)$ and $B-(0.441-0.217-0.342)$ corrosponding to THN-IBB- $n C_{12}$. 
The results of these calculations are reported in Table 4.2(a-b). Although the third mixture $\left(n_{T H N}\right)_{I B B} \mid n C_{12}$ can be obtained by subtracting the $\left(n_{n C_{12}}\right)_{I B B} \mid T H N$ mixture from $\left(n_{T H N}\right)_{n C_{12}} \mid I B B$, we prepared the mixtures and performed experimental measurements of the refractive index. The primary purpose for preparing and measuring the refractive indices of mixture three was to verify our measurement accuracy which was found in third order of magnitude in both cases.

Table 4.2: Calculated concentration and temperature coefficients of refractive indices of the two investigated ternary systems of THN-IBB- $n \mathrm{C}_{12}: \mathrm{a}-(\mathbf{0 . 5 0 1 - 0 . 1 1 0 - 0 . 3 8 9 )}$ and $\mathrm{b}-(0.441-0.217-0.342)$ at atmospheric pressure $\mathbf{P}=0.1 \mathrm{MPa} *$.

Table 4.2a

Refractive index Concentration Coefficient (dn/dc)THN |IBB

\begin{tabular}{|c|c|c|c|c|c|c|c|}
\hline $\begin{array}{l}\text { Wavelength } \\
(\mathrm{nm})\end{array}$ & $293 \mathrm{~K}$ & $298 \mathrm{~K}$ & $303 \mathrm{~K}$ & $308 \mathrm{~K}$ & $313 \mathrm{~K}$ & $318 \mathrm{~K}$ & \\
\hline 657.2 & 0.11634 & 0.11685 & 0.11668 & 0.11701 & 0.11681 & 0.11672 & $-4.65 \mathrm{E}-04$ \\
\hline $632.9^{a}$ & 0.11754 & 0.11724 & 0.11742 & 0.11755 & 0.11786 & 0.11738 & $-4.66 \mathrm{E}-04$ \\
\hline 589.2 & 0.11880 & 0.11892 & 0.11911 & 0.11941 & 0.11918 & 0.11905 & $-4.68 \mathrm{E}-04$ \\
\hline 513.9 & 0.12282 & 0.12289 & 0.12316 & 0.12324 & 0.12316 & 0.12304 & $-4.74 \mathrm{E}-04$ \\
\hline \multirow[t]{2}{*}{436.1} & 0.13053 & 0.13045 & 0.13094 & 0.13086 & 0.13074 & 0.13056 & $-4.86 \mathrm{E}-04$ \\
\hline & \multicolumn{6}{|c|}{ Refractive index Concentration Coefficient (dn/dc)IBB $\mid n C 12$} & $(\mathrm{dn} / \mathrm{dT}) \operatorname{IBB} \mid n \mathrm{C} 12$ \\
\hline 657.2 & 0.07085 & 0.07005 & 0.06932 & 0.06890 & 0.06844 & 0.06778 & $-4.63 \mathrm{E}-04$ \\
\hline $632.9^{\mathrm{a}}$ & 0.07317 & 0.07266 & 0.07223 & 0.07174 & 0.07123 & 0.07068 & $-4.62 \mathrm{E}-04$ \\
\hline 589.2 & 0.07187 & 0.07137 & 0.07068 & 0.07021 & 0.06959 & 0.06917 & $-4.63 \mathrm{E}-04$ \\
\hline 513.9 & 0.07578 & 0.07492 & 0.07431 & 0.07398 & 0.07361 & 0.07347 & $-4.70 \mathrm{E}-04$ \\
\hline \multirow[t]{2}{*}{436.1} & 0.08264 & 0.08222 & 0.08174 & 0.08150 & 0.08086 & 0.07992 & $-4.83 \mathrm{E}-04$ \\
\hline & \multicolumn{6}{|c|}{ Refractive index Concentration Coefficient (dn/dc)|BB |THN } & $(\mathrm{dn} / \mathrm{dT}) \mathrm{IBB} \mid \mathrm{THN}$ \\
\hline 657.2 & 0.04687 & 0.04724 & 0.04756 & 0.04796 & 0.04821 & 0.04926 & $-4.67 \mathrm{E}-04$ \\
\hline $632.9^{\mathrm{a}}$ & 0.04683 & 0.04722 & 0.04755 & 0.04792 & 0.04823 & 0.04877 & $-4.68 \mathrm{E}-04$ \\
\hline 589.2 & 0.04715 & 0.04764 & 0.04799 & 0.04827 & 0.04870 & 0.04888 & $-4.70 \mathrm{E}-04$ \\
\hline 513.9 & 0.04798 & 0.04839 & 0.04887 & 0.0490 & 0.04955 & 0.04986 & $-4.78-04$ \\
\hline 436.1 & 0.04965 & 0.05005 & 0.05050 & 0.05066 & 0.05122 & 0.05133 & $-4.89 E-04$ \\
\hline
\end{tabular}

Table $4.2 b^{a}$

\begin{tabular}{cccccccc} 
Wavelength & \multicolumn{6}{c}{ Refractive index Concentration Coefficient $(\mathrm{dn} / \mathrm{dc}) \mathrm{THN} \mid \mathrm{IBB}$} & \multirow{2}{*}{ (dn/dT)THN|IBB } \\
\cline { 2 - 6 }$n m$ & $293 \mathrm{~K}$ & $298 \mathrm{~K}$ & $303 \mathrm{~K}$ & $308 \mathrm{~K}$ & $313 \mathrm{~K}$ & $318 \mathrm{~K}$ & \\
\hline 657.2 & 0.11586 & 0.11628 & 0.11692 & 0.11749 & 0.11814 & 0.11859 & $-4.69 \mathrm{E}-04$ \\
$632.9^{\mathrm{b}}$ & 0.11802 & 0.11856 & 0.11903 & 0.11954 & 0.11987 & 0.12028 & $-4.58 \mathrm{E}-04$ \\
589.2 & 0.11697 & 0.11753 & 0.11810 & 0.11866 & 0.11909 & 0.11942 & $-4.69 \mathrm{E}-04$
\end{tabular}




\begin{tabular}{|c|c|c|c|c|c|c|c|}
\hline 513.9 & 0.12189 & 0.12284 & 0.12390 & 0.12536 & 0.12449 & 0.12568 & $-4.78 \mathrm{E}-04$ \\
\hline 436.1 & 0.12939 & 0.12948 & 0.13066 & 0.13061 & 0.13096 & 0.13132 & $-4.87 \mathrm{E}-04$ \\
\hline \multicolumn{7}{|c|}{ Refractive index Concentration Coefficient $(\mathrm{dn} / \mathrm{dc})$ IBB $\mid n \mathrm{C} 12$} & $(\mathrm{dn} / \mathrm{dT}) \mathrm{IBB} \mid n \mathrm{C} 12$ \\
\hline 657.2 & 0.07164 & 0.07077 & 0.07120 & 0.07211 & 0.07178 & 0.07122 & $-4.64 \mathrm{E}-04$ \\
\hline $632.9^{\mathrm{b}}$ & 0.06968 & 0.07098 & 0.06703 & 0.06629 & 0.06532 & 0.06492 & $-4.57 E-04$ \\
\hline 589.2 & 0.07246 & 0.07286 & 0.07333 & 0.07233 & 0.07213 & 0.07167 & $-4.67 \mathrm{E}-04$ \\
\hline 513.9 & 0.07561 & 0.07512 & 0.07698 & 0.07539 & 0.07510 & 0.07482 & $-4.74 \mathrm{E}-04$ \\
\hline 436.1 & 0.08291 & 0.08332 & 0.08338 & 0.08454 & 0.08367 & 0.08250 & $-4.86 \mathrm{E}-04$ \\
\hline \multicolumn{7}{|c|}{ Refractive index Concentration Coefficient $(\mathrm{dn} / \mathrm{dc}) \mathrm{IBB} \mid \mathrm{THN}$} & $(\mathrm{dn} / \mathrm{dT}) \mathrm{IBB} \mid \mathrm{THN}$ \\
\hline 657.2 & 0.04290 & 0.04464 & 0.04456 & 0.04465 & 0.04531 & 0.04849 & $-4.54 \mathrm{E}-04$ \\
\hline $632.9^{\mathrm{a}}$ & 0.04319 & 0.04483 & 0.04470 & 0.04483 & 0.04543 & 0.05662 & $-4.54 \mathrm{E}-04$ \\
\hline 589.2 & 0.04419 & 0.04583 & 0.04570 & 0.04583 & 0.04643 & 0.05462 & $-4.65 \mathrm{E}-04$ \\
\hline 513.9 & 0.04553 & 0.04820 & 0.04814 & 0.04819 & 0.04874 & 0.06001 & $-4.75 E-04$ \\
\hline 436.1 & 0.04624 & 0.05001 & 0.04982 & 0.05004 & 0.05055 & 0.05766 & $-4.84 \mathrm{E}-04$ \\
\hline
\end{tabular}

"The reported average expanded uncertainty $U=1.197 \times 10^{-3}$ was calculated based on the coverage factor $k=2$, providing a level of confidence of approximately $95 \%$. ${ }^{a}$ Current study against the literature [94] with average standard deviation of $1.6 \times 10^{-3}$. The average relative error of the temperature and concentration coefficients of the refractive index for the two investigated systems using both measurement methods are presented in table 3 and 4 .

\subsubsection{Mach-Zehnder Interferometer}

Another set of experiments were conducted on the same ternary mixtures using the MZI technique at a single wavelength of $632.9 \mathrm{~nm}$. Using the MZI technique, we measured the temperature derivative $(\partial n / \partial T)_{C_{o} \lambda}$ of the refractive indices of the $\mathrm{THN}, \mathrm{IBB}$ and $\mathrm{C}_{12}$ ternary mixtures at a constant concentration and wavelength. We also measured the concentration derivative $(\partial n / \partial C)_{T_{o} \lambda}$ of the refractive indices of the THN, IBB and $\mathrm{C}_{12}$ ternary mixtures at a constant temperature and wavelength. The experimental values of the phase change as a function of temperature and concentration of $\left(n_{T H N}\right)_{C_{12}} \mid I B B$, and $\left(n_{C_{12}}\right)_{I B B} \mid T H N$ were performed using $\operatorname{MatLab}{ }^{\circledR}$. The variations of refractive indices due the temperature and concentration gradient were calculated based on the unwrap phase change $\Delta \varphi$. The derivative of the refractive index with respect to temperature and concentration was calculated with $\Delta n=\frac{\lambda \Delta \varphi}{2 \pi L}$, where $\mathrm{L}$ is the working cell thickness $(L=10 \mathrm{~mm})$. The phase change due to the temperature gradient was obtained from the CCD camera images and extracted from the PID controller data. The phase change due to the concentration gradient was calculated by subtracting the starting reference sample concentration from the concentration changes in the second cell after injecting the preprepared mixture using a syringe pump. The primary advantage of the reference cell is that it helps eliminate any measurement errors caused by noise or vibration. 
We performed a quantitative comparison between the two experimental methods and found excellent agreement in both cases. The average relative error of the refractive index temperature coefficient for the two investigated systems was $7.6 \times 10^{-3}$ and $1.8 \times 10^{-2}$ for $\left(n_{T H N}\right)_{n C_{12}} \mid I B B$ by refractometer and $1.6 \times 10^{-2}$ and $5.4 \times 10^{-2}$ for $\left(n_{n C_{12}}\right)_{I B B} \mid T H N$ using MZI. The average relative error of the refractive index concentration coefficient was $2.5 \times 10^{-2}, \quad 1.6 \times 10^{-2}$ for $\left(n_{T H N}\right)_{n C_{12}} \mid I B B$, and $2.3 \times 10^{-2}, 1.7 \times 10^{-2}$ for $\left(n_{n C_{12}}\right)_{I B B} \mid T H N$. Table $4.3(\mathrm{a}, \mathrm{b})$ lists the thermal coefficients of refractive indices for various compositions and concentrations. Table $4.4(\mathrm{a}, \mathrm{b})$ lists the concentration derivative of refractive indices for various compositions and temperatures.

Table 4.3: Refractive index temperature coefficient of at different compositions of $\left(\mathbf{n}_{\mathrm{THN}}\right)_{\mathrm{IBB}}$, and $\left(\mathbf{n}_{\mathrm{IBB}}\right)_{\mathrm{THN}}$ at a wavelength of $632.9 \mathrm{~nm}$ conducted by refractometer and MZI with relative error for the ternary systems of: a- $(0.501-0.110-0.389)$ and b- $(0.441-0.217-0.342)$ corresponding to $\left(\mathrm{THN}-\mathrm{IBB}-n \mathrm{C}_{12}\right)$ at atmospheric pressure $\mathbf{P}=0.1 \mathrm{MPa}$.

Table 4.3a

\begin{tabular}{|c|c|c|c|c|c|c|c|}
\hline$(\mathrm{n})_{\mathrm{THN}} \mid \mathrm{IBB}$ & Refractometer & MZI & RE & (n) ${ }_{\mathrm{IBB}} \mid n \mathrm{C}_{12}$ & $\begin{array}{c}\text { Refractomete } \\
r\end{array}$ & $\mathrm{MZI}$ & RE \\
\hline $0.451-0.111$ & $-4.61 E-04$ & $-4.68 \mathrm{E}-04$ & 0.015 & $0.446-0.165$ & $-4.46 \mathrm{E}-04$ & $-4.62 E-04$ & 0.036 \\
\hline $0.461-0.111$ & $-4.61 E-04$ & $-4.65 \mathrm{E}-04$ & 0.007 & $0.457-0.154$ & $-4.50 \mathrm{E}-04$ & $-4.61 \mathrm{E}-04$ & 0.025 \\
\hline $0.471-0.111$ & $-4.67 \mathrm{E}-04$ & $-4.63 \mathrm{E}-04$ & 0.008 & $0.468-0.143$ & $-4.54 \mathrm{E}-04$ & $-4.60 \mathrm{E}-04$ & 0.014 \\
\hline $0.481-0.110$ & $-4.56 \mathrm{E}-04$ & $-4.60 \mathrm{E}-04$ & 0.010 & $0.479-0.132$ & $-4.57 \mathrm{E}-04$ & $-4.58 \mathrm{E}-04$ & 0.003 \\
\hline $0.491-0.110$ & $-4.56 \mathrm{E}-04$ & $-4.58 \mathrm{E}-04$ & 0.005 & $0.490-0.121$ & $-4.56 \mathrm{E}-04$ & $-4.58 \mathrm{E}-04$ & 0.003 \\
\hline $0.501-0.110$ & $-4.57 \mathrm{E}-04$ & $-4.57 \mathrm{E}-04$ & 0.002 & $0.501-0.110$ & $-4.54 \mathrm{E}-04$ & $-4.58 \mathrm{E}-04$ & 0.009 \\
\hline $0.510-0.109$ & $-4.56 \mathrm{E}-04$ & $-4.56 \mathrm{E}-04$ & 0.001 & $0.512-0.099$ & $-4.58 \mathrm{E}-04$ & $-4.57 \mathrm{E}-04$ & 0.003 \\
\hline $0.520-0.109$ & $-4.57 \mathrm{E}-04$ & $-4.55 \mathrm{E}-04$ & 0.003 & $0.523-0.088$ & $-4.57 \mathrm{E}-04$ & $-4.57 \mathrm{E}-04$ & 0.001 \\
\hline $0.530-0.108$ & $-4.56 \mathrm{E}-04$ & $-4.52 \mathrm{E}-04$ & 0.009 & $0.534-0.077$ & $-4.56 \mathrm{E}-04$ & $-4.54 \mathrm{E}-04$ & 0.004 \\
\hline $0.540-0.108$ & $-4.54 \mathrm{E}-04$ & $-4.51 \mathrm{E}-04$ & 0.007 & $0.545-0.066$ & $-4.65 E-04$ & $-4.53 E-04$ & 0.025 \\
\hline $0.550-0.108$ & $-4.54 \mathrm{E}-04$ & $-4.45 E-04$ & 0.019 & $0.556-0.055$ & $-4.72 \mathrm{E}-04$ & $-4.49 \mathrm{E}-04$ & 0.050 \\
\hline Average & & & 7.5983 & & & & $1.57 \mathrm{E}-02$ \\
\hline
\end{tabular}

Table 4.3b

\begin{tabular}{cccccccc}
\hline (n) THN $_{\text {IIBB }}$ & Refractometer & MZI & RE & $(n)_{\text {IBB }} \mid n C_{12}$ & Refractometer & MZI & RE \\
\hline $0.391-0.220$ & $-4.70 \mathrm{E}-04$ & $-4.73 \mathrm{E}-04$ & 0.005 & $0.386-0.272$ & $-4.68 \mathrm{E}-04$ & $-4.73 \mathrm{E}-04$ & 0.010 \\
$0.401-0.219$ & $-4.66 \mathrm{E}-04$ & $-4.69 \mathrm{E}-04$ & 0.005 & $0.397-0.261$ & $-4.74 \mathrm{E}-04$ & $-4.78 \mathrm{E}-04$ & 0.009 \\
$0.411-0.219$ & $-4.68 \mathrm{E}-04$ & $-4.67 \mathrm{E}-04$ & 0.002 & $0.408-0.250$ & $-4.76 \mathrm{E}-04$ & $-4.80 \mathrm{E}-04$ & 0.009 \\
$0.421-0.218$ & $-4.74 \mathrm{E}-04$ & $-4.63 \mathrm{E}-04$ & 0.025 & $0.419-0.239$ & $-4.71 \mathrm{E}-04$ & $-4.74 \mathrm{E}-04$ & 0.007 \\
$0.431-0.218$ & $-4.63 \mathrm{E}-04$ & $-4.60 \mathrm{E}-04$ & 0.006 & $0.430-0.228$ & $-4.76 \mathrm{E}-04$ & $-4.79 \mathrm{E}-04$ & 0.006
\end{tabular}




\begin{tabular}{lllllllc}
$0.441-0.217$ & $-4.57 \mathrm{E}-04$ & $-4.59 \mathrm{E}-04$ & 0.003 & $0.441-0.217$ & $-4.66 \mathrm{E}-04$ & $-4.68 \mathrm{E}-04$ & 0.004 \\
$0.451-0.217$ & $-4.64 \mathrm{E}-04$ & $-4.58 \mathrm{E}-04$ & 0.014 & $0.452-0.206$ & $-4.66 \mathrm{E}-04$ & $-4.68 \mathrm{E}-04$ & 0.003 \\
$0.461-0.216$ & $-4.69 \mathrm{E}-04$ & $-4.57 \mathrm{E}-04$ & 0.026 & $0.463-0.195$ & $-4.68 \mathrm{E}-04$ & $-4.70 \mathrm{E}-04$ & 0.004 \\
$0.470-0.215$ & $-4.64 \mathrm{E}-04$ & $-4.54 \mathrm{E}-04$ & 0.024 & $0.474-0.184$ & $-4.66 \mathrm{E}-04$ & $-4.68 \mathrm{E}-04$ & 0.003 \\
$0.480-0.215$ & $-4.69 \mathrm{E}-04$ & $-4.52 \mathrm{E}-04$ & 0.037 & $0.485-0.174$ & $-4.61 \mathrm{E}-04$ & $-4.62 \mathrm{E}-04$ & 0.003 \\
$0.490-0.214$ & $-4.69 \mathrm{E}-04$ & $-4.46 \mathrm{E}-04$ & 0.051 & $0.495-0.163$ & $-4.55 \mathrm{E}-04$ & $-4.56 \mathrm{E}-04$ & 0.002 \\
\hline Average & \multicolumn{7}{c}{$1.79 \mathrm{E}-02$} \\
\hline
\end{tabular}

We also performed a comparison between our results and those of the published research for validation purposes. In regards to the concentration dependence of refractive index measurements at 298k for the target mixture 0.501-0.110-0.389 found in the literature [15,94], the accuracy of our experimental measurements was in the third order of magnitude. For clarity, the wavelength used in the literature was $670 \mathrm{~nm}$ compared to the $657.2 \mathrm{~nm}$ used in this study.

Table 4.4: Refractive index concentration coefficient of at different compositions of $\left(n_{T H N}\right)_{I B B}$, and $\left(\mathrm{n}_{\mathrm{IBB}}\right)_{\mathrm{THN}}$ at a wavelength of $632.9 \mathrm{~nm}$ conducted by refractometer and MZI with relative error for the ternary systems of: a- $(0.501-0.110-0.389)$ and b- $(0.441-0.217-0.342)$ corresponding to $\left(\mathrm{THN}-\mathrm{IBB}-n \mathrm{C}_{12}\right)$.

Table 4.4a

\begin{tabular}{|c|c|c|c|c|c|c|}
\hline \multirow[b]{2}{*}{ Temp K } & \multicolumn{2}{|c|}{$(\mathrm{dn} / \mathrm{dc}) \mathrm{THN} \mid \mathrm{IBB}$} & \multirow[b]{2}{*}{$\mathrm{RE}$} & \multicolumn{2}{|c|}{$(\mathrm{dn} / \mathrm{dc})$ IBB $\mid n \mathrm{C}_{12}$} & \multirow[b]{2}{*}{ RE } \\
\hline & Refractometer & $\mathrm{MZI}$ & & Refractometer & $\mathrm{MZI}$ & \\
\hline 293 & 0.118 & 0.1223 & $3.52 \mathrm{E}-02$ & 0.0697 & 0.0705 & $1.14 \mathrm{E}-02$ \\
\hline 298 & 0.1186 & 0.1223 & 3.03E-02 & 0.071 & 0.069 & $2.90 \mathrm{E}-02$ \\
\hline 303 & 0.119 & 0.1223 & $2.70 \mathrm{E}-02$ & 0.067 & 0.0685 & 2.19E-02 \\
\hline 308 & 0.1195 & 0.1223 & $2.29 \mathrm{E}-02$ & 0.0663 & 0.0673 & 1.49E-02 \\
\hline 313 & 0.1199 & 0.1223 & $1.96 \mathrm{E}-02$ & 0.0653 & 0.0673 & 2.97E-02 \\
\hline 318 & 0.1203 & 0.1223 & $1.64 \mathrm{E}-02$ & 0.0649 & 0.0668 & $2.84 \mathrm{E}-02$ \\
\hline Average & & & $2.52 \mathrm{E}-02$ & & & $2.25 \mathrm{E}-02$ \\
\hline
\end{tabular}

Table 4.4b

\begin{tabular}{cccccccc} 
& \multicolumn{2}{c}{$(\mathrm{dn} / \mathrm{dc}) \mathrm{THN} \mid \mathrm{IBB}$} & & \multicolumn{3}{c}{$(\mathrm{dn} / \mathrm{dc})|\mathrm{BB}| n \mathrm{C}_{12}$} \\
\cline { 2 - 4 } \cline { 6 - 8 } Temp K & Refractometer & $\mathrm{MZI}$ & RE & & Refractometer & MZI \\
\hline 293 & 0.1175 & 0.1199 & $2.00 \mathrm{E}-02$ & 0.0468 & 0.0479 & $2.27 \mathrm{E}-02$ \\
298 & 0.1172 & 0.1192 & $1.68 \mathrm{E}-02$ & 0.0472 & 0.0481 & $1.87 \mathrm{E}-02$ \\
303 & 0.1174 & 0.1194 & $1.68 \mathrm{E}-02$ & 0.0476 & 0.0484 & $1.65 \mathrm{E}-02$ \\
308 & 0.1176 & 0.1192 & $1.34 \mathrm{E}-02$ & 0.0479 & 0.0486 & $1.44 \mathrm{E}-02$ \\
313 & 0.1179 & 0.1193 & $1.17 \mathrm{E}-02$ & 0.0482 & 0.0489 & $1.43 \mathrm{E}-02$ \\
318 & 0.1174 & 0.1186 & $1.01 \mathrm{E}-02$ & 0.0488 & 0.0494 & $1.22 \mathrm{E}-02$ \\
\hline Average & & & $1.48 \mathrm{E}-02$ & & & $1.65 \mathrm{E}-02$ \\
\hline
\end{tabular}




\subsection{Theoretical Calculations}

Refractive indices can also be estimated theoretically using suitable "mixture rule" equations such as the Lorentz-Lorenz, Gladstone-Dale, Arago-Biot, Eykman, Wiener, Heller, Newton, Lichtenecker and Oster equations. All of these are theoretically derived rules based upon the electromagnetic theory of light. These equations can only be applied if there are no chemical reactions in the mixture and if there are no changes in volume during mixing. If these conditions are met, these relations can be used to predict the refractive indices of multi-component mixtures based on the knowledge of some of the physical properties of the pure components used in the mixture. The present study used these mixing rules in order to predict the refractive indices of the ternary mixtures under study. These theoretical estimations were also used to verify and validate the experimental measurements. The present study made use of some of the relations to predict the refractive indices of the ternary mixtures under study. In addition, the predictions obtained were used to verify and validate the experimental measurements.

The most widely used equations are listed below. The Lorentz-Lorenz (L-L) relation is applied most often in the evaluation of the refractive indices of mixture and their pure components. The formula is as follows:

$$
\left[\frac{n^{2}-1}{n^{2}+2}\right]=\sum_{i=1}^{N}\left[\frac{n_{i}^{2}-1}{n_{i}^{2}+2}\right] \varphi_{i}
$$

The second most widely used equation is the Goldstone-Dale (G-D). The formula is as follows:

$$
n-1=\sum_{i=1}^{N}\left[n_{i}-1\right] \varphi_{i}
$$

Assuming volume additivity, Arango-Biot (A-B) proposed the following equation to evaluate the refractive index of a ternary mixture in the form:

$$
n=\sum_{i=1}^{N}\left(n_{i}\right) \varphi_{i}
$$

Similarly, the Wiener (W) and Heller $(\mathrm{H})$ relations for multi-component systems are written as follows:

$$
\frac{n^{2}-n_{1}^{2}}{n^{2}+2 n_{1}^{2}}=\sum_{i \neq 1}^{N}\left[\frac{n_{i}^{2}-n_{1}^{2}}{n_{i}^{2}+2 n_{1}^{2}}\right] \varphi_{i}
$$


and

$$
\frac{n-n_{1}}{n_{1}}=\frac{3}{2} \sum_{i \neq 1}^{N}\left[\frac{\left(n_{i} / n_{1}\right)^{2}-1}{\left(n_{i} / n_{1}\right)^{2}+2}\right] \varphi_{i}
$$

Newton $(\mathrm{N})$ proposed another equation that can be written as:

$$
n^{2}-1=\sum_{i=1}^{N}\left[n_{i}^{2}-1\right] \varphi_{i}
$$

While the Eykman (E) relation is expressed as:

$$
\frac{n^{2}-1}{n+0.4}=\sum_{i=1}^{N}\left[\frac{n_{i}^{2}-1}{n_{i}+0.4}\right] \varphi_{i}
$$

The Lichtenecker equation is expressed as:

$$
\ln n=\sum_{i=1}^{N} \varphi_{i} \ln n_{i}
$$

The Oster $(\mathrm{O})$ equation is expressed as:

$$
\frac{(n-1)\left(2 n^{2}+1\right)}{n^{2}}=\sum_{i=1}^{N} \frac{\left(n_{i}^{2}-1\right)\left(2 n_{i}^{2}+1\right)}{n_{i}^{2}} \varphi_{i}
$$

And the Looyenga $\mathrm{H}[99]$. (LH) expression as:

$$
\left[n^{2 / 3}-1\right]=\sum_{i=1}^{N}\left[n_{i}^{2 / 3}-1\right] \varphi_{i}
$$

where $n$ is the refractive index of the mixture and $n_{i}$ is that of $i^{t h}$ pure component. $\varphi_{i}$ is the volume fraction of $\mathrm{i}^{\text {th }}$ component that can be obtained by the relation

$$
\varphi_{i}=x_{i} V / \sum_{i=1}^{N} x_{i} V_{i}
$$

And $\varphi_{\mathrm{i}}=\omega_{\mathrm{i}} / \rho_{\mathrm{i}}$ where $\rho_{\mathrm{i}}$ is the density of the $\mathrm{i}^{\text {th }}$ component and the mixture. $\omega_{\mathrm{i}}$ is the weight fraction of the $\mathrm{i}^{\text {th }}$ component. 
We conducted a comparison of the refractive indices obtained using the Abbemat Refractometer and the predicted values using some of the mixing rules discussed above and the results of these calculations are reported in Table $S 4(a-b)$ in the supplementary material. The estimated values of the refractive indices were found to be in good agreement with the experimental results, with the distinction of Wiener, Looyenga and Lorentz-Lorenz which revealed better agreement, with two decimal place order of magnitude, when compared to Newton and Oster expression. the relative error found between the experimental and theoretical models of the $\left(n_{T H N}\right)_{C_{12}} \mid I B B$ mixture at $298 \mathrm{~K}$ and different wavelengths for the two systems under examination was used as an example in Figure 4-6 which depicts the comparison between the proposed experimental values and the theoretical values obtained using the mixing rule equations with the average relative error of $9.1 \times 10^{-3}$ and $1.1 \times 10^{-2}$ rrespectively. The results indicate good agreement and the proposed data are sufficiently accurate.
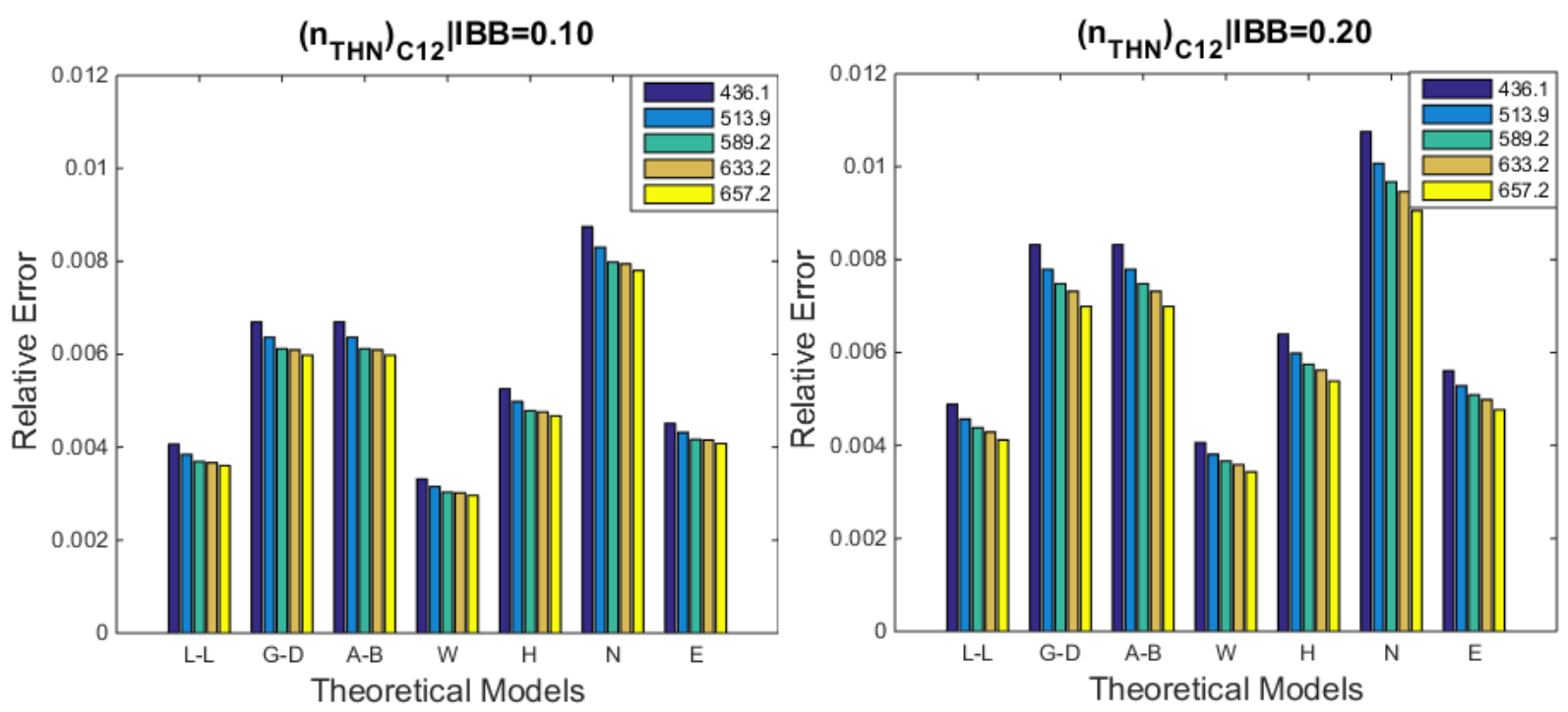

Figure 4-6: Relative error of the experimental and the estimated values of refractive index obtained with mixing rules for various compositions of $(\mathrm{nTHN}) \mathrm{nC12} \mid \mathrm{IBB}$ at a single temperature of $298 \mathrm{~K}$ with respect to the two investigated systems: A- $(0.501-0.110-0.389)$ and $B-(0.441-0.217-0.342)$ corrosponding to THN-IBB- $n C_{12}$. 


\subsection{Conclusions}

New improvements on the copper block cell holder currently used in the Mach-Zehnder Interferometer technique to measure the contrast factors in transparent liquids were presented and tested on ternary hydrocarbon mixtures. We conducted organized and highly accurate experimental measurements of the refractive indices of two ternary systems consisting of $n$ Dodecane $\left(n \mathrm{C}_{12}\right)$, Isobutylbenzene (IBB), and Tetrahydronaphtalene (THN) with respect to concentration $(\partial \mathrm{n} / \partial \mathrm{C})$ and temperature $(\partial \mathrm{n} / \partial \mathrm{T})$ using refractometer and interferometer techniques at different wavelength. The obtained measurements suggest a linear dependence with regards to concentration and temperature and a non-linear dependence with regards to wavelengths. These results support past findings in the literature. There was excellent agreement between both techniques, suggesting that the contrast factors of transparent liquids can be measured using any of these techniques with high accuracy. The results were also in agreement with the experimental results found in the published literature.

The measured experimental refractive indices were compared with those estimated by applying several mixing rules: Lorentz-Lorenz (L-L), Looyenga (LH), Gladstone-Dale (G-D), Arago-Biot (A-B), Eykman (E), Wiener (W), Newton (N), and Oster (O). The experimental values of refractive indices are in good agreement with the values obtained by W, L-L, L, H, GD, A-B and E equations excepting those obtained by the Oster and Newton mixing rules.. Using a wide range of temperatures and wavelengths for each ternary system allows researchers to obtain more information, increasing the depth of scientific knowledge and advancing scientific research. 


\section{CHAPTER 5 EMPIRICAL MODELLING TO PREDICT THE REFRACTIVE INDEX OF HUMAN BLOOD}

This chapter is based on the following published paper

Yahya M., Saghir M. Z. "Empirical Modelling to Predict the Refractive Index of Human Blood", Phys Med Biol, 2015, 00,1-11.

\subsection{Introduction}

Quantitative information about the optical properties of blood has always been of great interest in the field of medical diagnostics because it provides information regarding the patient's condition as well as therapeutic applications in laser medicine [100]. These properties are the key parameters in the development of mathematical models describing light propagation in biological tissues and are an essential part of the development of new optical techniques used in the field of biology and medicine. This knowledge is also important to pre-clinical trials and pharmaceutical development [101].

Blood is one of the most important connective tissues within the human body. Optical measurement techniques are suitable candidates for the measurement of the properties of blood as they are non-invasive and capable of providing highly accurate measurements on a "nanometre scale" without disturbing the cells; however, they are difficult to perform due to the nature of the blood itself. Blood is a heterogeneous medium consisting of cells suspended in plasma. Blood plasma is composed of water (90\%) and protein (10\%) while the blood cells consist primarily of Erythrocytes (Red blood cells (RBCs); almost 99\%), leukocytes (White blood cells; $1 \%$ ) and platelets [39,102-104]. The optical parameters of blood depend mainly on the optical properties of its components. These are described using the scattering coefficient, absorption coefficient and refractive index. The scattering and absorption coefficients of the medium for a given wavelength $\lambda$ represent a probability, per unit of length, of a photon being scattered or absorbed. The refractive index describes the change in the speed of light when propagating through a medium, compared to the speed of light in a vacuum. This optical behaviour occurs as a result of the highly concentrated hemoglobin content of erythrocyte cells. In general terms, the optical effects of blood are determined by its optical properties such as the complex refraction index, the size distribution and the shape of RBCs. The complex index of refraction $n=n_{R e l}+i n_{i m}$ consists of a real portion $n_{R e l}$, which represents the ratio of the speed 
of light in a vacuum to the speed of light in the material $n_{\text {Rel }}=c / v$, and an imaginary portion $n_{i m}$, which represents an absorption parameter characterizing the material. The imaginary part of the refractive index can be understood using the Bouguer-Lambert law. The attenuation of light passing through the medium is linearly related to the distance the light travels through the medium $x$ and the concentration of the absorber $\mathrm{C}$ by the given expression $A=$ $\epsilon C x$ where $\epsilon$ represents the molar absorptivity. Likewise, the absorbance $A$ can be determined by the relation $A=-\log T$ where $\mathrm{T}$ is the transmission given by $I / I_{O}$ is the ratio between the transmitted and incident light intensities. Consequently, for a bulk material, the ratio of the intensity $I$ at some point in the material to the incident intensity $\mathrm{I}_{\mathrm{o}}$ is obtained using $I / I_{o}=e^{-k x}$. This equation is known as Beer's absorption law. $k=\epsilon C$ represents the Beer absorption coefficient, which is characteristic of the bulk material. For the bulk material, $n_{i m}$ is related to the Beer absorption coefficient $k$ by means of $n_{i m}=k \lambda / 4 \pi$.

The hemoglobin, which is mainly concentrated in the RBCs, causes the real portion of the refractive index to be higher than the surrounding plasma. The imaginary portion is described by absorption measurements and is well documented by several researchers [105,106]. Nevertheless, simplified models such as the Kubelka-Munk model [107] have been widely used in the past to determine the absorption and scattering coefficients of biological media. In recent years, the Monte-Carlo simulation technique has been used in order to estimate the optical properties of biological samples [108].

Measuring the real portion of the refractive index is a complex task, especially at physiological or near physiological RBC concentration (haematocrit). Furthermore, refractive index values vary widely in the literature [109]. Several researchers have proposed indirect methods of deriving the real portion of the refractive index from the imaginary portion. For example, the optical parameters of RBCs in circulating human blood have been determined using integrating sphere measurements followed by inverse Monte-Carlo simulations [110,111]. Other methods calculate the optical properties from the complex refractive index of RBCs using Mie's theory or the Rayleigh-Gans theory [112] and using the Kramers-Kronig relations [113,114]. Only a few theoretical and experimental studies of the optical properties of blood have led to reasonable results because the optical properties of blood depend primarily on physiological and biochemical parameters such as haematocrit, temperature and wavelength. 
An extensive literature search revealed that there are no available mathematical models based on empirical data. This study examined the refractive index of human blood at various hemoglobin concentrations, temperatures and wavelengths using the Abbemat Refractometer.

The experimental measurements were conducted using a human blood mimicking consisting of dry human hemoglobin dissolved in isotonic phosphate buffered saline (PBS) to maintain the PH at 7.4. The blood mimicking phantom is widely used in the literature as an alternative human blood substance that provides a homogenous hemoglobin concentration across the test sample equivalent to human blood at that concentration and shows better stability of the experimental optical measurements [109,114,115].

Analysis of the experimental data suggested an empirical model function to estimate the refractive index of the human blood based on knowledge of the hemoglobin concentration, temperature and wavelength. The proposed model was developed based on empirical modelling using linear- regression analysis in the Matlab ${ }^{\circledR}$ environment. A comparison between the model and the results available in the literature revealed that the new model is able to predict the real portion of refractive index of human blood with an acceptable level of accuracy.

\subsection{Materials and Experimental Method}

Dry human hemoglobin 'Lyophilized powder H7123' supplied by Sigma-Aldrich® was used in this study as a human blood mimicking phantom. The powder was dissolved in PBS purchased from the same supplier to keep the PH constant at 7.4 and exclude any changes in refractive index due to changes in PH value. Sixteen samples were prepared with a haematocrit between 1 and $150 \mathrm{~g} / \mathrm{L}$ based on pre-prescribed mass fraction with a sampling rate of $10 \%$. The prepared test samples for every concentration were $20 \mathrm{~g}(20 \mathrm{ml})$ each in order to reduce the volume of the samples. The samples were charged in leak proof caps in order to reduce sample evaporation. The prescribed mass was measured using a Denver Instrument ${ }^{\circledR}$ SI-234 electronic balance, with a precision of $\pm 1 \times 10^{-3} \mathrm{~g} / 20 \mathrm{~g}$. The samples were prepared individually on a magnetic stirrer and the required Lyophilized powder was added gradually to prevent the conglomeration and to ensure the homogeneity of the solution. In order to ensure measurement accuracy, the samples were used immediately following preparation.

The Abbemat refractometer is a standard method used to measure the refractive index of liquids [94,116]. The digital multi-wavelength Anton Paar ${ }^{\circledR}$ WR Refractometer was used to 
measure the refractive index of the samples under investigation. The working principle of the device is based on Snell's Law [88] which determines the critical angle of the total internal reflection of the investigated sample. The reflected beam is then detected by a sensor array, and the refractive index of the sample can be calculated. This technique makes the device suitable for the measurement of the refractive index of heavy/dense liquids. In order to perform the desired measurements, the sample is placed in a sample mould on top of the YAG prism (YttriumAluminum-Garnet) that irradiates from different angles via an LED light. In this study, a highpower LED was used as the light source along with an interference filter yielding a wavelength error of $0.1 \mathrm{~nm}$. The prism temperature (of the sample under investigation) was controlled by a Peltier thermostat with a temperature stability of $\pm 2 \times 10^{-3} \mathrm{~K}$ and a refractive index accuracy of \pm $4 \times 10^{-5}$.

The instrument was calibrated using double distilled water with a specific conductance of $2.0 \mu \mathrm{mho} / \mathrm{cm}$ at $296 \mathrm{~K}$ prior to each measurement series. The calibrations were carried out in accordance with a specifications table supplied by the refractometer manufacturer. The instrument was cleaned and dried with Acetone lab wipes after each calibration and sample measurement. Each sample was placed on a magnetic stirrer for several minutes prior to measurement in order to ensure the homogeneity of the sample. The measurements were carried out by setting the refractometer temperature to $293 \mathrm{~K}$ and a few drops of the sample were placed on the refractometer prism, once the digital reading of the refractometer was obtained for each wavelength $(657.2,633.2,589.2,513.9$ and 436.1) $\mathrm{nm}$, the temperature was gradually increased up to $318 \mathrm{~K}$ in $5 \mathrm{~K}$ intervals and the refractive indices were recorded for the aforementioned range of temperatures and wavelengths. The total number of measurements was 480 (16 samples $\times 6$ different temperatures $\times 5$ wavelengths).

All measurements were repeated three times within the same condition to increase measurement accuracy. The average standard uncertainty of the repeated measurements $u_{r}=$ $\frac{S D}{\sqrt{n}}(S D$ represents the standard deviation for the number of measurements $n)$, calculated based on the mean values of the repeatability of refractive index measurements, was $5.19 \times 10^{-4}$. The verification of the accuracy and the repeatability of the refractive index determination was accomplished by calculating the average expanded uncertainty using the given expression $U=k u_{c}$ where $u_{c}=u_{r}+u_{i}$ is the total standard uncertainty. The estimated average expanded 
uncertainty was $2.34 \times 10^{-3}$, calculated based the coverage factor $k=2$, providing a level of confidence of approximately $95 \%$.

\subsection{Results and Discussion}

The experimental measurements were conducted on mimicking phantom hemoglobin samples using the Abbemat Refractometer. This substance shows better stability compared to human blood in experimental measurement conditions. Sixteen human blood mimicking samples with various hematocrits (erythrocyte volume fraction (EVF)) were prepared and examined. The refractive indices of the investigated samples were measured using the Abbemat Refractometer technique for five different wavelengths $\lambda=(436.1,513.9,589.3,632.9$ and 657.2) $\mathrm{nm}$ and six different temperatures $\mathrm{T}=(293.15$ to 318.15$) \mathrm{K}$. The results are gathered in Table 5.1. The results revealed a strong linear dependence between the refractive index and total hemoglobin concentration. This is in agreement with the results found in the literature [117,118]. Barer has proven that for many substances in solution, the index of refraction correlates linearly with the concentration of the formula:

$$
n_{\text {Rel }}=n_{o}+\alpha C
$$

where $n_{o}$ represents the refractive index of solvent "PBS", $\alpha$ represents the specific refraction increment (in $\mathrm{mL} / \mathrm{g}$ ) which depends on the nature of the solute "Hemoglobin" and $C$ represents the concentration of dry solute in the solution (in $\mathrm{g} / \mathrm{mL}$ ).

Table 5.1: The real part of the refractive index of human blood mimicking phantom for various

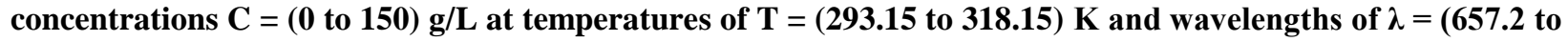
436) $\mathrm{nm}$. The table also presents the specific refraction concentrations and temperature increments $\alpha, \beta$ respectively.

\begin{tabular}{|c|c|c|c|c|c|c|c|}
\hline \multirow{2}{*}{$\begin{array}{c}\text { Hb C } \\
\text { g/L }\end{array}$} & \multicolumn{6}{|c|}{ Wavelength $=657.2 \mathrm{~nm}^{\mathrm{a}}$} & \multirow[t]{2}{*}{$\beta$} \\
\hline & $293.15^{\text {a }}$ & 298.15 & 303.15 & 308.15 & 313.15 & 318.15 & \\
\hline 0 & 1.33279 & 1.33231 & 1.33175 & 1.33113 & 1.33064 & 1.32968 & $-1.210 \mathrm{E}-04$ \\
\hline 10 & 1.33449 & 1.33397 & 1.33357 & 1.33312 & 1.33255 & 1.33186 & $-1.021 \mathrm{E}-04$ \\
\hline 20 & 1.33573 & 1.33535 & 1.33492 & 1.33439 & 1.33377 & 1.33323 & $-1.015 \mathrm{E}-04$ \\
\hline 30 & 1.33716 & 1.33677 & 1.33637 & 1.33586 & 1.33524 & 1.33462 & $-1.017 \mathrm{E}-04$ \\
\hline 40 & 1.33838 & 1.33795 & 1.33739 & 1.33689 & 1.33665 & 1.33574 & $-1.006 \mathrm{E}-04$ \\
\hline 50 & 1.34005 & 1.33993 & 1.33911 & 1.33866 & 1.33806 & 1.33729 & $-1.135 \mathrm{E}-04$ \\
\hline 60 & 1.34153 & 1.34117 & 1.34086 & 1.34042 & 1.33989 & 1.33923 & $-9.017 \mathrm{E}-05$ \\
\hline
\end{tabular}




\begin{tabular}{|c|c|c|c|c|c|c|c|}
\hline 70 & 1.34361 & 1.34315 & 1.34257 & 1.34208 & 1.34171 & 1.34101 & $-1.018 \mathrm{E}-04$ \\
\hline 80 & 1.34514 & 1.34485 & 1.34435 & 1.34361 & 1.34335 & 1.34273 & $-9.880 \mathrm{E}-05$ \\
\hline 90 & 1.34632 & 1.34657 & 1.34544 & 1.34514 & 1.34462 & 1.34394 & $-1.032 \mathrm{E}-04$ \\
\hline 100 & 1.34752 & 1.34778 & 1.34675 & 1.34697 & 1.34616 & 1.34554 & $-8.309 \mathrm{E}-05$ \\
\hline 110 & 1.34889 & 1.34923 & 1.34891 & 1.34854 & 1.34763 & 1.34725 & $-7.640 \mathrm{E}-05$ \\
\hline 120 & 1.35061 & 1.35043 & 1.35063 & 1.34981 & 1.34903 & 1.34846 & $-9.011 \mathrm{E}-05$ \\
\hline 130 & 1.35247 & 1.35204 & 1.35182 & 1.35124 & 1.35054 & 1.34967 & $-1.090 \mathrm{E}-04$ \\
\hline 140 & 1.35421 & 1.35376 & 1.35322 & 1.35287 & 1.35243 & 1.35113 & $-1.128 \mathrm{E}-04$ \\
\hline 150 & 1.35587 & 1.35524 & 1.35484 & 1.35427 & 1.35373 & 1.35312 & $-1.077 \mathrm{E}-04$ \\
\hline$\alpha 1 / g$ & $1.521 \mathrm{E}-04$ & $1.535 \mathrm{E}-04$ & $1.545 \mathrm{E}-04$ & $1.549 \mathrm{E}-04$ & $1.539 \mathrm{E}-04$ & $1.537 \mathrm{E}-04$ & \\
\hline \multicolumn{8}{|c|}{ Wavelength $=632.9 \mathrm{~nm}^{\mathrm{a}}$} \\
\hline 0 & 1.33342 & 1.33293 & 1.33237 & 1.33174 & 1.33104 & 1.33030 & $-1.253 \mathrm{E}-04$ \\
\hline 10 & 1.33500 & 1.33456 & 1.33419 & 1.33351 & 1.33301 & 1.33226 & $-1.088 \mathrm{E}-04$ \\
\hline 20 & 1.33646 & 1.33595 & 1.33544 & 1.33493 & 1.33435 & 1.33386 & $-1.046 \mathrm{E}-04$ \\
\hline 30 & 1.33783 & 1.33746 & 1.33696 & 1.33647 & 1.33581 & 1.33516 & $-1.072 \mathrm{E}-04$ \\
\hline 40 & 1.33902 & 1.33849 & 1.33799 & 1.33743 & 1.33678 & 1.33613 & $-1.152 \mathrm{E}-04$ \\
\hline 50 & 1.34089 & 1.34057 & 1.33956 & 1.33910 & 1.33852 & 1.33802 & $-1.198 \mathrm{E}-04$ \\
\hline 60 & 1.34233 & 1.34186 & 1.34115 & 1.34081 & 1.34014 & 1.33961 & $-1.091 \mathrm{E}-04$ \\
\hline 70 & 1.34408 & 1.34361 & 1.34300 & 1.34263 & 1.34219 & 1.34138 & $-1.037 \mathrm{E}-04$ \\
\hline 80 & 1.34557 & 1.34512 & 1.34465 & 1.34419 & 1.34366 & 1.34296 & $-1.023 \mathrm{E}-04$ \\
\hline 90 & 1.34652 & 1.34667 & 1.34612 & 1.34573 & 1.34478 & 1.34423 & $-1.002 \mathrm{E}-04$ \\
\hline 100 & 1.34805 & 1.34796 & 1.34752 & 1.34747 & 1.34625 & 1.34601 & $-8.783 \mathrm{E}-05$ \\
\hline 110 & 1.34950 & 1.34953 & 1.34942 & 1.34876 & 1.34773 & 1.34782 & $-8.259 \mathrm{E}-05$ \\
\hline 120 & 1.35111 & 1.35078 & 1.35112 & 1.35003 & 1.34933 & 1.34913 & $-8.772 \mathrm{E}-05$ \\
\hline 130 & 1.35289 & 1.35242 & 1.35242 & 1.35181 & 1.35075 & 1.35026 & $-1.073 \mathrm{E}-04$ \\
\hline 140 & 1.35456 & 1.35396 & 1.35389 & 1.35345 & 1.35273 & 1.35184 & $-1.013 \mathrm{E}-04$ \\
\hline 150 & 1.35601 & 1.35553 & 1.35528 & 1.35475 & 1.35401 & 1.35336 & $-1.048 \mathrm{E}-04$ \\
\hline$\alpha 1 / \mathrm{g}$ & $1.495 \mathrm{E}-04$ & $1.504 \mathrm{E}-04$ & $1.545 \mathrm{E}-04$ & $1.544 \mathrm{E}-04$ & $1.520 \mathrm{E}-04$ & $1.535 \mathrm{E}-04$ & \\
\hline \multicolumn{8}{|c|}{ Wavelength $=589.1 \mathrm{~nm}^{\mathrm{a}}$} \\
\hline 0 & 1.33465 & 1.33416 & 1.33358 & 1.33294 & 1.33224 & 1.33150 & $-1.265 \mathrm{E}-04$ \\
\hline 10 & 1.33624 & 1.33578 & 1.33525 & 1.33483 & 1.33412 & 1.33333 & $-1.143 \mathrm{E}-04$ \\
\hline 20 & 1.33763 & 1.33719 & 1.33667 & 1.33617 & 1.33559 & 1.33498 & $-1.061 \mathrm{E}-04$ \\
\hline
\end{tabular}




\begin{tabular}{|c|c|c|c|c|c|c|c|}
\hline 30 & 1.33905 & 1.33859 & 1.33814 & 1.33757 & 1.33695 & 1.33638 & $-1.076 \mathrm{E}-04$ \\
\hline 40 & 1.34017 & 1.33977 & 1.33928 & 1.33869 & 1.33807 & 1.33743 & $-1.109 \mathrm{E}-04$ \\
\hline 50 & 1.34213 & 1.34173 & 1.34105 & 1.34050 & 1.33972 & 1.33931 & $-1.181 \mathrm{E}-04$ \\
\hline 60 & 1.34334 & 1.34296 & 1.34259 & 1.34203 & 1.34144 & 1.34053 & $-1.095 \mathrm{E}-04$ \\
\hline 70 & 1.34524 & 1.34466 & 1.34442 & 1.34389 & 1.34312 & 1.34245 & $-1.093 \mathrm{E}-04$ \\
\hline 80 & 1.34676 & 1.34634 & 1.34598 & 1.34525 & 1.34476 & 1.34403 & $-1.093 \mathrm{E}-04$ \\
\hline 90 & 1.34812 & 1.34794 & 1.34760 & 1.34701 & 1.34612 & 1.34557 & $-1.075 \mathrm{E}-04$ \\
\hline 100 & 1.34964 & 1.34909 & 1.34914 & 1.34820 & 1.34766 & 1.34732 & $-9.627 \mathrm{E}-05$ \\
\hline 110 & 1.35104 & 1.35053 & 1.35081 & 1.34974 & 1.34922 & 1.34886 & $-9.086 \mathrm{E}-05$ \\
\hline 120 & 1.35258 & 1.35214 & 1.35256 & 1.35128 & 1.35063 & 1.35041 & $-9.507 \mathrm{E}-05$ \\
\hline 130 & 1.35418 & 1.35369 & 1.35378 & 1.35284 & 1.35219 & 1.35184 & $-9.799 \mathrm{E}-05$ \\
\hline 140 & 1.35578 & 1.35516 & 1.35536 & 1.35463 & 1.35393 & 1.35332 & $-9.554 \mathrm{E}-05$ \\
\hline 150 & 1.35724 & 1.35676 & 1.35679 & 1.35592 & 1.35530 & 1.35474 & $-1.015 \mathrm{E}-04$ \\
\hline$\alpha 1 / \mathrm{g}$ & $1.509 \mathrm{E}-04$ & $1.505 \mathrm{E}-04$ & $1.567 \mathrm{E}-04$ & $1.531 \mathrm{E}-04$ & $1.533 \mathrm{E}-04$ & $1.555 \mathrm{E}-04$ & \\
\hline \multicolumn{8}{|c|}{ Wavelength $=513.9 \mathrm{~nm}$} \\
\hline 0 & 1.33733 & 1.33683 & 1.33625 & 1.33560 & 1.33489 & 1.33413 & $-1.284 \mathrm{E}-04$ \\
\hline 10 & 1.33896 & 1.33849 & 1.33795 & 1.33758 & 1.33684 & 1.33625 & $-1.077 \mathrm{E}-04$ \\
\hline 20 & 1.34034 & 1.33996 & 1.33942 & 1.33886 & 1.33825 & 1.33761 & $-1.103 \mathrm{E}-04$ \\
\hline 30 & 1.34168 & 1.34125 & 1.34079 & 1.34028 & 1.33970 & 1.33912 & $-1.026 \mathrm{E}-04$ \\
\hline 40 & 1.34303 & 1.34247 & 1.34203 & 1.34137 & 1.34073 & 1.33996 & $-1.216 \mathrm{E}-04$ \\
\hline 50 & 1.34495 & 1.34451 & 1.34365 & 1.34320 & 1.34258 & 1.34184 & $-1.248 \mathrm{E}-04$ \\
\hline 60 & 1.34608 & 1.34568 & 1.34528 & 1.34465 & 1.34411 & 1.34333 & $-1.089 \mathrm{E}-04$ \\
\hline 70 & 1.34783 & 1.34737 & 1.34695 & 1.34630 & 1.34592 & 1.34493 & $-1.114 \mathrm{E}-04$ \\
\hline 80 & 1.34980 & 1.34886 & 1.34853 & 1.34785 & 1.34745 & 1.34663 & $-1.186 \mathrm{E}-04$ \\
\hline 90 & 1.35135 & 1.35051 & 1.35041 & 1.34942 & 1.34928 & 1.34863 & $-1.046 \mathrm{E}-04$ \\
\hline 100 & 1.35268 & 1.35162 & 1.35197 & 1.35123 & 1.35043 & 1.35028 & $-9.320 \mathrm{E}-05$ \\
\hline 110 & 1.35406 & 1.35329 & 1.35382 & 1.35262 & 1.35241 & 1.35183 & $-8.566 \mathrm{E}-05$ \\
\hline 120 & 1.35545 & 1.35471 & 1.35564 & 1.35457 & 1.35408 & 1.35361 & $-6.949 \mathrm{E}-05$ \\
\hline 130 & 1.35709 & 1.35642 & 1.35729 & 1.35561 & 1.35572 & 1.35482 & $-8.646 \mathrm{E}-05$ \\
\hline 140 & 1.35901 & 1.35813 & 1.35869 & 1.35701 & 1.35722 & 1.35641 & $-9.949 \mathrm{E}-05$ \\
\hline 150 & 1.36053 & 1.35968 & 1.35985 & 1.35859 & 1.35883 & 1.35769 & $-1.029 \mathrm{E}-04$ \\
\hline$\alpha 1 / g$ & $1.545 \mathrm{E}-04$ & $1.511 \mathrm{E}-04$ & $1.610 \mathrm{E}-04$ & $1.537 \mathrm{E}-04$ & $1.594 \mathrm{E}-04$ & $1.585 \mathrm{E}-04$ & \\
\hline
\end{tabular}




\begin{tabular}{cccccccc}
\multicolumn{7}{c}{ Wavelength $=\mathbf{4 3 6 . 1} \mathbf{~ n m}$} \\
0 & 1.34194 & 1.34143 & 1.34083 & 1.34016 & 1.33944 & 1.33866 & $-1.319 \mathrm{E}-04$ \\
10 & 1.34378 & 1.34331 & 1.34270 & 1.34213 & 1.34150 & 1.34070 & $-1.225 \mathrm{E}-04$ \\
20 & 1.34516 & 1.34476 & 1.34426 & 1.34362 & 1.34311 & 1.34239 & $-1.111 \mathrm{E}-04$ \\
30 & 1.34671 & 1.34623 & 1.34576 & 1.34507 & 1.34468 & 1.34405 & $-1.064 \mathrm{E}-04$ \\
40 & 1.34789 & 1.34754 & 1.34712 & 1.34642 & 1.34591 & 1.34536 & $-1.042 \mathrm{E}-04$ \\
50 & 1.35031 & 1.34930 & 1.34850 & 1.34824 & 1.34789 & 1.34726 & $-1.129 \mathrm{E}-04$ \\
60 & 1.35140 & 1.35093 & 1.35056 & 1.34976 & 1.34941 & 1.34849 & $-1.139 \mathrm{E}-04$ \\
70 & 1.35291 & 1.35260 & 1.35229 & 1.35141 & 1.35113 & 1.35036 & $-1.033 \mathrm{E}-04$ \\
80 & 1.35462 & 1.35427 & 1.35399 & 1.35306 & 1.35276 & 1.35156 & $-1.188 \mathrm{E}-04$ \\
90 & 1.35581 & 1.35538 & 1.35538 & 1.35409 & 1.35428 & 1.35269 & $-1.154 \mathrm{E}-04$ \\
100 & 1.35702 & 1.35641 & 1.35678 & 1.35551 & 1.35579 & 1.35441 & $-9.246 \mathrm{E}-05$ \\
110 & 1.35834 & 1.35781 & 1.35839 & 1.35682 & 1.35731 & 1.35576 & $-9.126 \mathrm{E}-05$ \\
120 & 1.36006 & 1.35934 & 1.36013 & 1.35839 & 1.35888 & 1.35737 & $-9.469 \mathrm{E}-05$ \\
130 & 1.36148 & 1.36121 & 1.36138 & 1.36003 & 1.36039 & 1.35879 & $-9.863 \mathrm{E}-05$ \\
140 & 1.36335 & 1.36272 & 1.36321 & 1.36142 & 1.36213 & 1.36039 & $-1.049 \mathrm{E}-04$ \\
150 & 1.36481 & 1.36434 & 1.36449 & 1.36322 & 1.36389 & 1.36211 & $-9.211 \mathrm{E}-05$ \\
\hline$\alpha 1 / \mathrm{g}$ & $1.498 \mathrm{E}-04$ & $1.496 \mathrm{E}-04$ & $1.582 \mathrm{E}-04$ & $1.521 \mathrm{E}-04$ & $1.601 \mathrm{E}-04$ & $1.515 \mathrm{E}-04$ & \\
\hline $\mathrm{T}$ & & & & & \\
\hline
\end{tabular}

\footnotetext{
The reported average expanded uncertainty $U=2.34 \times 10^{-3}$ was calculated based on the coverage factor $k=2$, providing a level of confidence of approximately $95 \%$.

${ }^{a}$ Standard deviation (SD) of $5.56 \times 10^{-4}$ between the measured refractive index for the wavelength of 657,533 and $589 \mathrm{~nm}$ at $293 \mathrm{k}$ and their corresponding concentration available in the literature [109].
}

This formula is based on the Gladstone and Dale law's and provides an approximation of the experimental results. This formula gives the relation between the refractive index and concentration with a degree of inaccuracy no greater than the inherent error of the refractometer under the conditions used in this investigation [119]. The refractive index is also correlated with wavelength and temperature in addition to concentration. The temperature effects in the range of measurement were small, yet non-negligible. Based on the experimental data, the values of $\alpha$ and $n_{o}$, at a constant wavelength, were determined using the linear least-squares method in Matlab. The linear model is obtained using the following expression

$$
n_{R e l}=n_{o}+\alpha C+\beta T
$$


where the estimated coefficients are $n_{o}=1.36$ average of $(1.355,1.378), \alpha=1.538 \times 10^{-4}$ of $\left(1.471 \times 10^{-4}, 1.605 \times 10^{-4}\right)$ and $\beta=-1.046 \times 10^{-4}$ of $\left(1.409 \times 10^{-4}, 6.82 \times 10^{-5}\right)$, representing the refractive index of the solvent and the concentration and temperature coefficients of the refractive index (respectively), with 95\% confidence bounds and goodness of fit SSE $=5.714 \times 10^{-}$ ${ }^{3}$ and R-square 0.98 . The concentrations of human hemoglobin, taken as mass fraction, and the temperature were plotted against refractive indices Figure 5-1a. As shown in the figure, within the limits of experimental error, the curve is a straight line. Although the refractive indices of human blood within the therapeutic window of the electromagnetic spectrum (400 to $1000 \mathrm{~nm}$ ) vary slightly between oxygenated and deoxygenated hemoglobin at a visible wavelength $[117,118]$, there appeared to be no significant difference between the hemoglobin mimicking substance used in the study and the results found in the literature [109]. The data obtained in this study can then be considered for both oxygenated and deoxygenated hemoglobin.

Additionally, non-linear behaviour variations in refractive indices with regard to wavelength were observed in all of the samples [119].
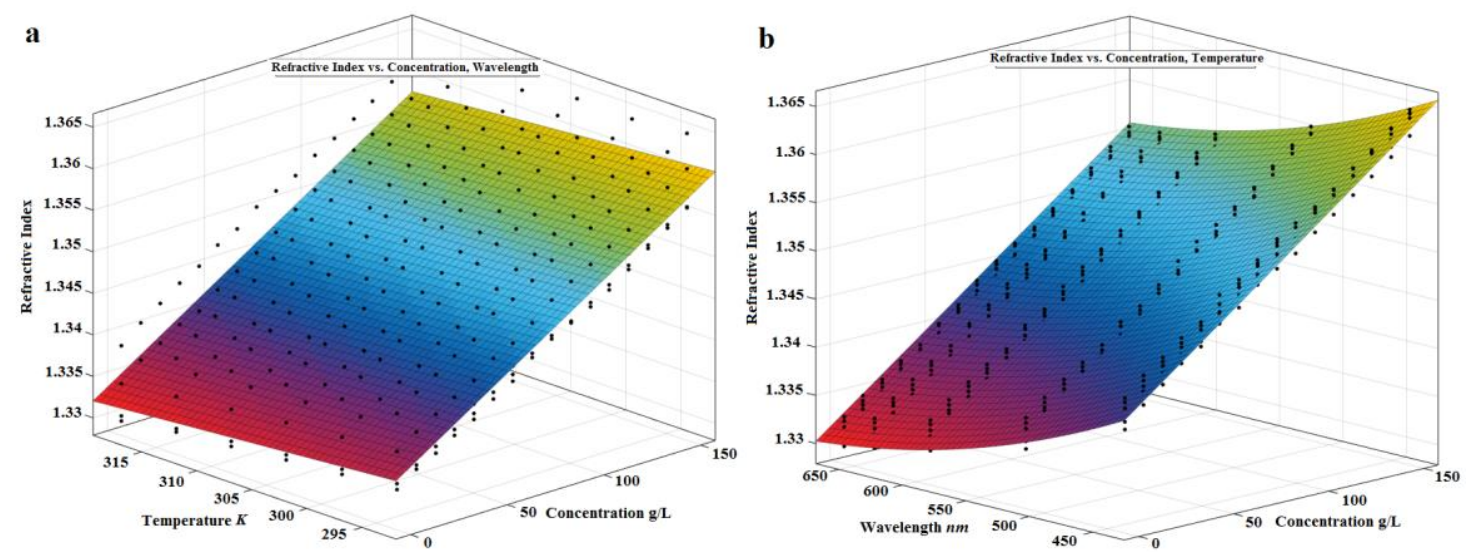

Figure 5-1: Carve fitting plot showing the refractive index as a function of a- temperature and concentration, b- wavelength and concentration.

Specifically, refractive index increases in a non-linear fashion as the wavelength decreases. This is due to electromagnetic interactions between the particles of light and particles within the medium, reducing the velocity of light in the medium and causing a higher refractive index 
$n_{R e l}=\mathrm{c} / \mathrm{v}$. This behaviour is similar to the well-known Cauchy dispersion formula [120]. The general expression below was used to calculate the refractive index at five different wavelengths.

$$
n_{R e l}=\mathrm{A}+\mathrm{B} / \lambda_{\mathrm{i}}{ }^{2}+\mathrm{C} /{\lambda_{\mathrm{i}}{ }^{4}}+\mathrm{D} / \lambda_{\mathrm{i}}{ }^{6}+\mathrm{E} / \lambda_{\mathrm{i}}{ }^{8}+\mathrm{F} / \lambda_{\mathrm{i}}{ }^{10}
$$

where $n_{\lambda_{i}}$ gives the refractive index at $\lambda_{i}$ wavelength, and $\mathrm{A}$ to $\mathrm{F}$ represent coefficients that can be determined for a given sample at a known wavelength. For instance, the coefficients A to F for the sample of $50 \mathrm{~g} / \mathrm{L}$ hemoglobin concentration at $298.15 \mathrm{~K}$ were calculated and found to be $1.6167,-4 \times 10^{-5}, 5.2362 \times 10^{10},-1.0009 \times 10^{16}$ and $7.0019 \times 10^{20}$ respectively. The Matlab ${ }^{\circledR}$ second $^{2}$ order polynomial fit was utilized to estimate the coefficients of such behaviour. At constant temperature, the Barer formula provided in Eq.5.1 was modified to the expression below

$$
n_{\text {Rel }}=n_{o}+\alpha_{1} C+\beta T+\gamma_{1} \lambda+\alpha_{2} C^{2}+\delta C \lambda+\gamma_{2} \lambda^{2}
$$

the estimated coefficients are $n_{o}=1.394$ average of $(1.389,1.399), \alpha_{1}=1.553 \times 10^{-4}$ of $\left(1.406 \times 10^{-4}, 1.7 \times 10^{-4}\right), \beta=-1.765 \times 10^{-4}$ of $\left(1.96 \times 10^{-4}, 1.571 \times 10^{-4}\right), \gamma_{1}=9.122 \times 10^{-9}$ of $\left(-3.609 \times 10^{-8}, 5.433 \times 10^{-8}\right), \alpha_{2}=-5.105 \times 10^{-9}$ of $\left(-2.793 \times 10^{-8}, 1.772 \times 10^{-8}\right)$ and $\gamma_{2}=1.236 \times$ $10^{-7}$ of $\left(1.059 \times 10^{-7}, 1.413 \times 10^{-7}\right)$ respectively, with $95 \%$ confidence bounds and goodness of fit $\mathrm{SSE}=4.299 \times 10^{-4}$ and R-square 0.985 . The fitted curve of the refractive index as a function of concentration and wavelength is illustrated in Figure 5-1b. Lastly, the Cauchy equation coefficients were verified using the Matlab® second polynomial fitting functions where the average fit was $7.105 \times 10^{-16}$.

\subsection{Mathematical Correlation}

While the available technologies make it simple to measure the refractive index of liquid samples, obtaining the hemoglobin concentration remains a challenging task. The majority of the work on refractive index measurements has been carried out on protein solutions of low concentration. Despite the fact that the refractive index of a solution can be estimated using Barer's law Eq. 5.1, the refractive index is correlated with wavelength and temperature in addition to concentration. The development of a mathematical model that allows for the estimation of hemoglobin concentration of a blood sample using the known refractive index (and vice versa) is essential in the medical sciences. An extensive literature review revealed that no study has evaluated a mathematical correlation based on experimental measurements of refractive indices across a wide range of hemoglobin concentrations, temperatures and 
wavelengths. In this study, linear-regression analysis was used to develop a mathematical model based on the experimental refractive index values of the sixteen samples under investigation. Linear regression is a common method used to identify the parameters of a model by solving an optimization problem. The least-square method, which minimizes the error between the predicted output values and the experimental input values, was used to find the optimal model parameters. The final expression of the optimal parameter values is

$$
\hat{\theta}=\left(X^{T} X\right)^{-1} X^{T} Y=\left[\sum_{k=1}^{N} x(k) x^{T}(k)\right]^{-1}\left[\sum_{k=1}^{N} x(k) y(k)\right]
$$

where $\mathrm{N}$ and $\mathrm{x}(\mathrm{k})$ represent the number of input dependent variables $\mathrm{x}(\mathrm{k})$ and $x^{T}(k)$ represents the transpose. Using this equation, we can estimate the optimal values for the model parameters. The final model is giving by

$$
n_{R e l}=n_{o}+\alpha C+\beta T+\gamma_{1} \lambda+\gamma_{2} \lambda^{2}+\gamma_{3} \lambda^{3}
$$

where, $C$ represents the mass fraction of the dry hemoglobin $\mathrm{g} / \mathrm{L}, \mathrm{T}$ represents the temperature in Kelvin units and $\lambda$ represents the wavelength in $\mathrm{nm}$. The model coefficients of $\left(\mathrm{n}_{0}, \alpha, \beta, \gamma_{1}, \gamma_{2}\right.$ and $\left.\gamma_{3}\right)$ are $\left(1.54712,2.413 \times 10^{-4},-6.497 \times 10^{-5},-8.47 \times 10^{-4}, 1.172 \times 10^{-4}\right.$, and $\left.-5.271 \times 10^{-10}\right)$ respectively. The first two coefficients of this equation represent the average refractive index of the solvent "PBS" and the specific refraction increment of the solute "Hemoglobin" (Barer's equation). The other coefficients are the suggestion added to Barer's expression that satisfies the temperature and the wavelength effects in the range of the experiment.

The real part of refractive index values obtained using the proposed model at physiological hemoglobin concentrations of $355.5 \mathrm{~g} / \mathrm{L}$ at $293.15 \mathrm{~K}$ and the PBS at zero hemoglobin concentration were compared to those available in the literature [117,121], and plotted against each other in Figure 5-2. The comparison revealed good agreement with an average standard deviation of $2.34 \times 10^{-3}$ and $1.64 \times 10^{-3}$ respectively. 


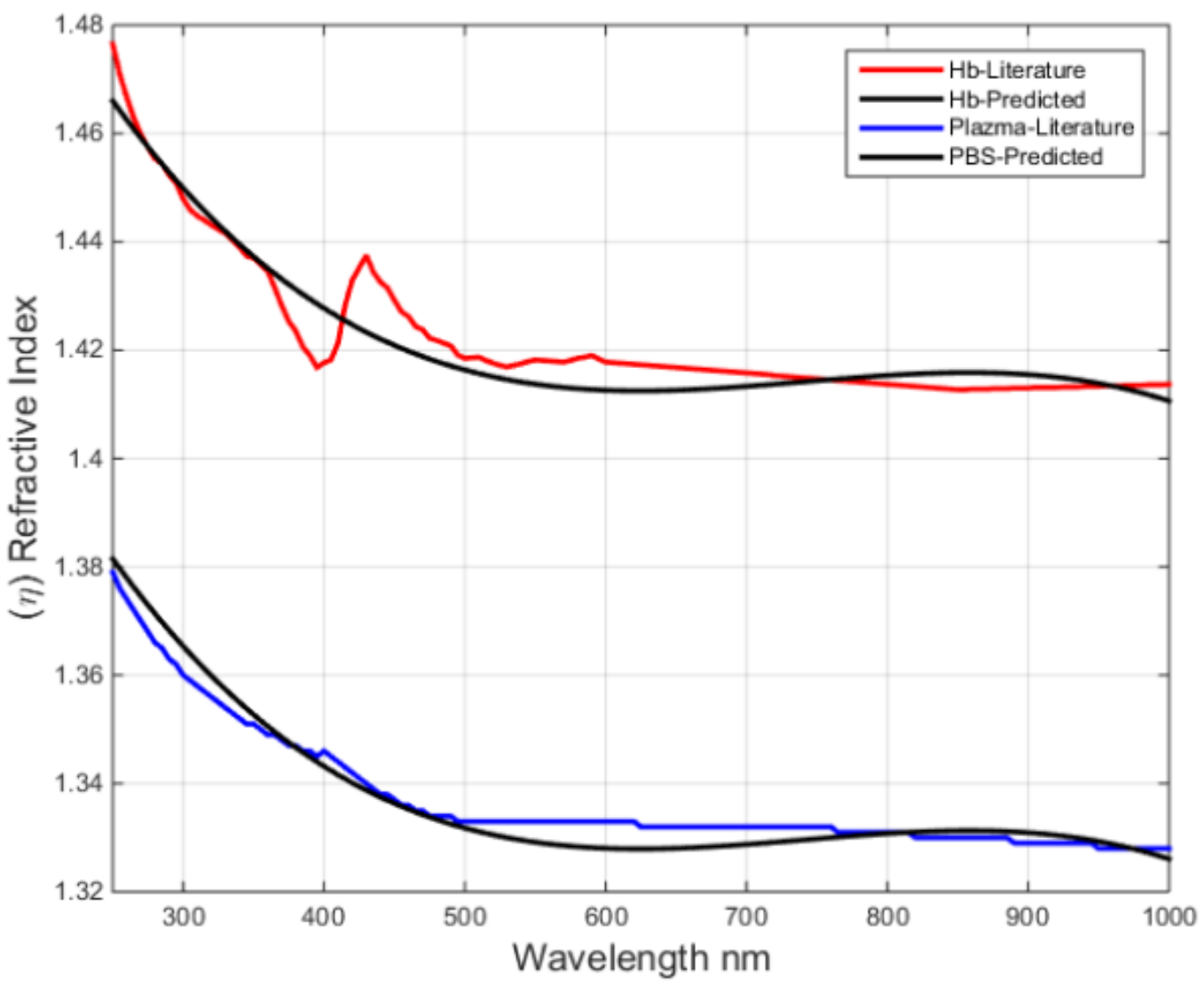

Figure 5-2 Predicted values of the real part of the refractive index of $\mathrm{Hb}$ mimicking phantom solutions at the concentration of 355.5 and $0 \mathrm{~g} / \mathrm{l}$ in the wavelength range 250-1000 $\mathrm{nm}$, and the two RI values for the $\mathrm{Hb}$ and the plasma found in the literature.

The anomalous dispersion and sudden increases of the refractive index near the ultra-violate spectrum corresponds to the high absorption band at these wavelengths. For instance, the maximum absorption of endogenous chromophores includes nucleic acids at $260 \mathrm{~nm}$, standard amino acids tryptophan at $260 \mathrm{~nm}$ and hemoglobin at $420 \mathrm{~nm}$. This is because the photon energy at this wavelength matches the difference between the excited and ground states of an electron in the biological materials $[122,123]$.

This behaviour is not observed using the human blood mimicking phantom due to the fact that the shortened wavelength used in the experimental measurement $(436.1 \mathrm{~nm})$ surpasses this anomalous dispersion. 


\subsection{Conclusions}

The real part of the refractive indices of human hemoglobin mimicking liquid samples of multiple-concentrations, temperatures and wavelengths were measured using an Abbemat Refractometer. Sixteen samples were carefully prepared based on pre-described mass fraction and examined with high accuracy. The results of the experimental measurements revealed that hemoglobin concentration plays an important role in determining the refractive index. Specifically, there is a linear relationship between the refractive index and hemoglobin concentration. The refractive index of the hemoglobin samples is also temperature-dependent, although the temperature effect on the refractive index is small when compared to the hemoglobin concentration effect. This behaviour can be used to predict the hemoglobin concentration of blood sample based on the knowledge of the refractive index and using the refraction increment provided.

The results of the investigation were utilized to develop a model based on linear regression that can be used to estimate the refractive index of human hemoglobin solutions as a function of concentration, temperature and wavelength. The real part of the complex refractive index can be determined for hemoglobin concentration up to physiological values within the RBCs at required temperatures and wavelengths. The proposed model added new temperature and wavelength factors into the commonly used Barer formula. The model was validated with the available literature which confirmed its ability to determine blood parameters used in medicine (such as hematocrit value). The average accuracy of the correlation when compared with our experimental values was $1.05 \times 10^{-3}$. The average accuracy of the correlation when compared to the results available in previous literature was $2.34 \times 10^{-3}$. 


\section{CHAPTER 6 QUANTITATIVE INTERFEROMETRIC MEASUREMENTS OF THE REFRACTIVE INDEX OF THE ERYTHROCTES}

This chapter is based paper under process:

Yahya M., Saghir M. Z. "Quantitative Interferometric Measurements of the Refractive Index of the RBCs", J Biomed Sci, 2015.

\subsection{Introduction}

Erythrocytes, also known as red blood cells (RBCs), are the main component of human blood and represent almost $99 \%$ of all blood cells. RBCs are produced by the bone marrow and live for about 120 days in the human circulatory system. The RBCs are responsible for transporting oxygen around the body. RBCs contain a high level of hemoglobin which binds easily with oxygen. RBCs are round, biconcave, disc-shaped cells with a thick rim and a thin sunken centre (minimum thickness $T_{\min }=0.81 \pm 0.35 \mu \mathrm{m}$ and maximum thickness $T_{\max }=$ $2.58 \pm 0.27 \mu \mathrm{m})[102,103]$, with typical geometrical dimensions of 6.2-8.2 $\mu$ [39]. Mature RBCs have a particular type of structure: they lack nuclei and major organelles. The biconcave, disc-shaped morphology and elasticity of RBC's are considered the principal contributors to the cell's ability to change form and travel through microvascular networks that are slightly smaller than them.

The high hemoglobin content of RBCs is primarily responsible for their refractive index. Therefore, the refractive index can independently describe the optical properties and health condition of RBCs. Knowledge of the physical properties of RBCs is of great interest in the fields of medicine and technology. These properties are essential in the design and development of new measurement instruments and also the refractive index of RBCs can be used to describe the patient's condition in the diagnostic or therapeutic stages. An unusual number of particular types of cells can indicate abnormalities, blood cell disorders or disease. Various types of malaria and anemia [124,125], and disorders such as Thrombotic Thrombocytopenic Purpura (TTP) can be detected and diagnosed at earlier stages using the blood cells images, allowing for medical decisions to be made immediately [126]. RBCs are also the most commonly transfused blood component and their refractive index can help determine the quality of blood transfusions [127131].

Surveys of the literature reveal that storage procedures and storage time cause morphological changes in RBCs [132]. Some studies have proposed that RBC quality (in terms 
of safety and efficiency) is decreased in proportion to prolonged storage time [133-136]. Little is known about the structural transformations that take place throughout the storage time.

Numerous techniques have been developed based on the principles of interferometry to gain information about the optical path length of the sample in order to reveal structures that would normally be invisible. The most commonly used methods include phase contrast microscopy (PCM) [137], differential interference contrast microscopy (DIC), digital holographic microscopy [45-49], Hilbert phase microscopy [50,51], diffraction phase microscopy [52], tomographic phase microscopy, [53], the spatial phase-shifting method [54,139] and spatial light interference microscopy (SLIM) [139-140].

Thin layers of most biological tissues and living cells are transparent and do not absorb or scatter light; therefore, they are not clearly visible under a bright field microscope. The working principle behind most of these methods is based on the deflection of light of the specimen under investigation and/or blood stains (e.g. MGG (May- Grünwald -Giemsa) method) [41,42].

Quantitative phase imaging (QPI) is a modern optical imaging technique with the ability to quantify cell morphology and optical properties using 3D imaging with nanoscale sensitivity. This method is non-invasive and label free [141-144]. The image produced by QPI is represented by the phase distribution of transparent and semitransparent specimens (low absorption and scattering properties) and a map of the path-length shifts associated with the particular specimen from which the refractive index and morphological structure can be obtained [78]. As light penetrates through a biological cell or tissue, there are deformations in the light wavefront in regions where cells are present. This deformation occurs in accordance with the refractive index of the cells compared to the surrounding medium. By analysing the phase shift induced in the optical path-length within the field of view we can obtain quantitative information about the specimen under investigation. The optical behaviour of blood is primarily determined by the optical properties of RBCs. The microphysical properties of blood (mainly RBC morphology and refractive index) allow for the evaluation of the medical state of the blood being examined.

In this study, interferometric system that is capable of providing precise measurements of the refractive indices of transparent and semi-transparent mediums was designed and developed. This system also provides real time full-field quantitative imaging with high temporal and spatial resolution for the study of biological cells. The interferogram obtained using the interferometric system can be analyzed based on the phase displacements with accuracy close to that of the laser 
light source wavelength. This study attempted to provide "in vitro" 3D imaging and experimental measurements using intrinsic cellular contrast such as the refractive index of RBCs. This information provides the opportunity for high throughput and real-time analysis of the mechanical properties of RBCs in healthy and diseased individuals.

The proposed system can also be downsized into a standalone blood smear analyzer used as a

point-of-care or bedside medical diagnostic micro-interferometric tool without relying on external measurements of hemoglobin concentration. This system requires minimal operational time, effort and expertise, allowing for immediate clinical decisions

Furthermore, a computer program was developed and used to extract, count and calculate the surface area of the RBCs from the interferogram. The results can be used to estimate the RBC concentration (hematocrit) at the physiological level. Further development and validation of this system is needed in order to correlate 3D structural changes with signalling events evaluated by traditional diagnostic techniques. In addition this technique can also be used in cancer diagnosis and tumor imaging [145].

\subsection{Experimental Method}

Interferometry is the science of combining light waves to cause interference. As two beams of light occupy the same space at the same time they tend to overlap (interfere) with one another at some point, causing areas of constructive and destructive interference $[61,62,81,146,147]$. This interference manifests as a series of bright and dark bands in 2-D space, known as fringes. The movement of the interference bands is called a fringe shift. The fringe shifts can be used to quantify the parameters that modify the path length of light waves. These parameters include the refractive index, the geometry of the system and other properties that are a function of these parameters such as temperature, pressure and concentration. The thickness of each fringe at any given point represents a path length equal to the wavelength of the light used to create the fringe. Common practice is therefore to measure the fringe shift based on the number of fringes to pass a certain point on the observation screen. This number is directly related to changes in the physical path of the light which can be used to quantify a multitude of physical phenomena.

There are several common types of interferometers used in both research and industry. The most common two-beam interferometers are the Michelson and Mach-Zehnder interferometers (MZI). The working principle of the Michelson interferometer is based on the light reflection of the investigated specimen commonly used in phase microscopy. 
The MZI configuration requires a collimated coherence light source with a combination of two beam splitters with $50 \%$ reflectivity and two high reflection mirrors aligned at specific angles, generally at 45 degrees or less (square or rectangular geometry). The MZI configuration, selected in this study, has a few advantages when compared to the Michelson configuration. First, the light beam passes through the sample once, making it a good candidate for biological tissues that have large aberrations. Second, the interferometer is sometimes automatically compensates because it uses two beam splitters.

A single $15 \mathrm{~mW}$ coherence visible red light with a maximum wavelength of $632.9 \mathrm{~nm}$ emitted from a He-Ne laser (CVI Melles Griot ${ }^{\circledR}$ 25-LHP-151-249) was utilized as the light source for the experiments. The visibility of a light source beam with a very small diameter (in the range of 1-2 $\mathrm{mm}$ ) provides a significant advantage for the optical apparatus alignment during the preparation stage. A schematic drawing of the setup used in this study is shown in Figure 6-1.

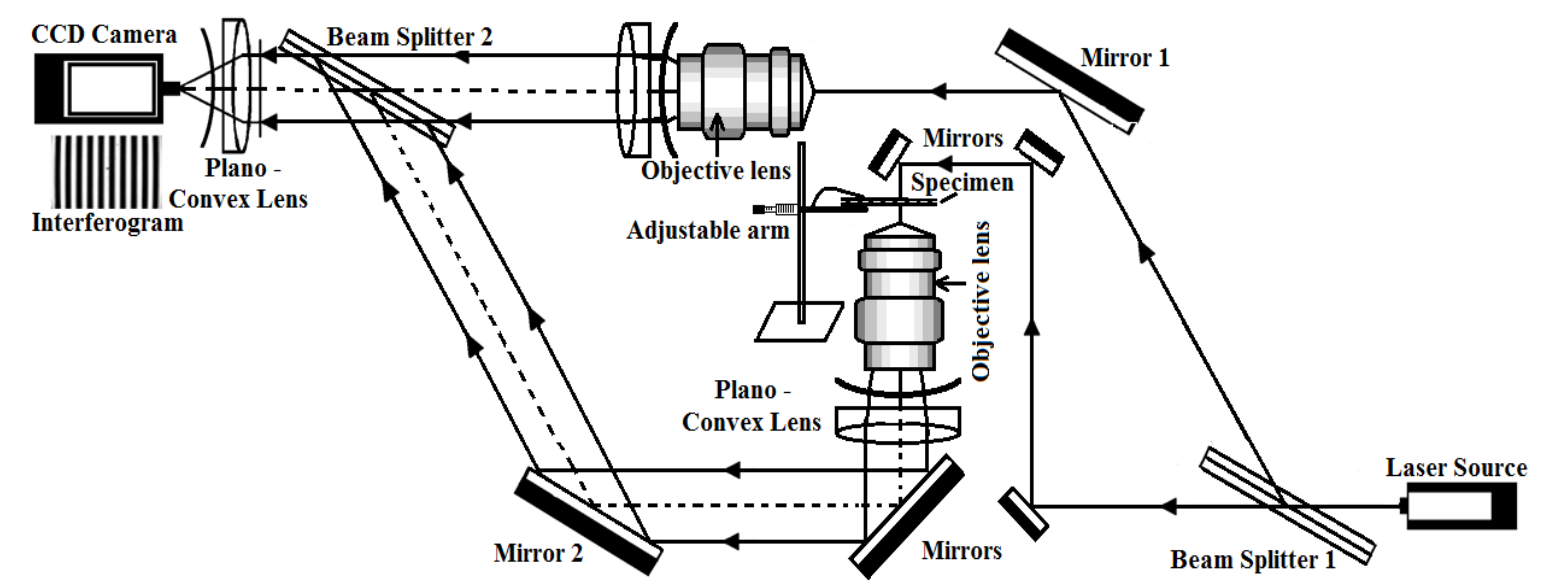

Figure 6-1: A schematic drawing of the experimental setup used in this study.

A polarized light filter was placed in front of the laser source to reduce light intensity in order to avoid sensor damage or saturation of the CCD camera. The laser beam was split into two identical intensities using a non-polarized beam splitter BS1. One of the beams was used as a reference beam while the other was passed through the specimen under investigation (objective beam).

Two microscopic objective lenses (20X, $N A=0.40$, Focal Length $f=9.0 \mathrm{~mm}$ ) were used to magnify the specimen. The beams expanded by the microscopic objective lenses were collimated into parallel rays using two Plano-Convex lenses with a focal length of $f=3.5 \mathrm{~cm}$. The two 
beams interfered and generated a spatially modulated interference image using the second beam splitter BS2 based on Mach-Zehnder configuration, which was then captured by a CCD camera (interferogram). The whole set up was mounted on a 'RS2000 series' tuned vibration damping optical table (Newport Corporation, dimension $1.2 \times 2.4 \times 0.203 \mathrm{~m}$, model: M-RS2000-48-8). The interference patterns were recorded with a CCD camera (JAI progressive scan, model: CVM4 CL+ monochrome digital megapixel camera) directly connected to a computer. The resolution of the CCD camera is $1280 \times 1024$ pixels.

\subsection{Theoretical Principles}

Interference phase imaging is a beneficial imaging tool that provides higher quality 3D image contrast and displays phase shifts induced in the optical path-length of an investigated specimen, allowing for nanometre-scale measurements of structures and cell dynamics. Thin layers of biological tissue are normally transparent to light. The speed of light penetrating through a transparent object will be reduced by a factor of $n$ which represents the object's refractive index. Thus, the optical path length of the object beam differs from the reference beam depending on the thickness and refractive index of the object under investigation. Optical path variation can appear as a delay or deformation of the incident light wavefront see Figure 6-2.

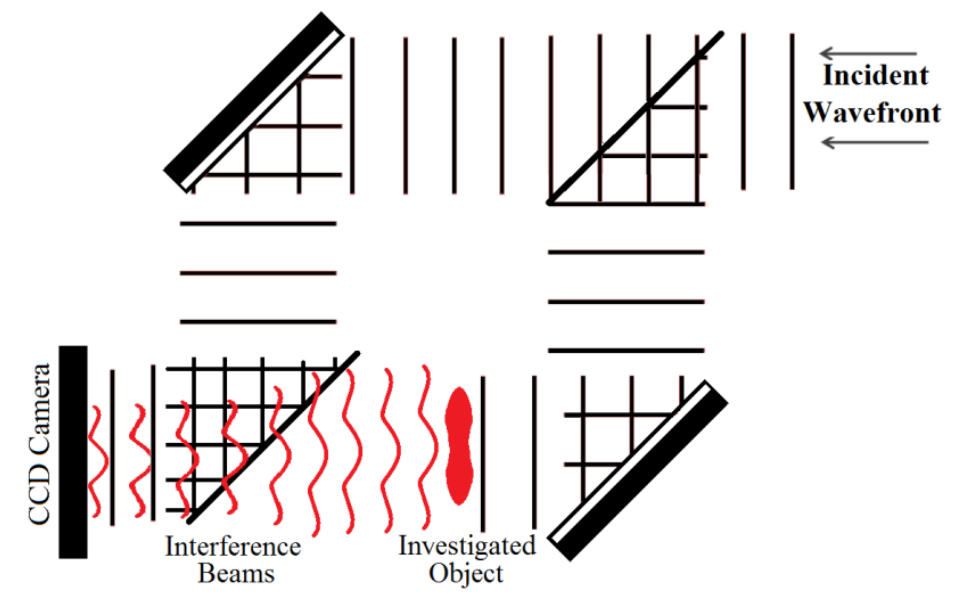

Figure 6-2: Quantitative phase imaging based on the Mach-Zehnder Interferometer. Phase delay occurred due to waves passing through the object under investigation.

A refractive index varying along the $\mathrm{z}$ direction caused by a change in optical path length and thereby a phase variation between a beam of light passing through the investigated object index (object beam) and another beam of light passing through a constant refractive index 
(reference beam) is related to the cell thickness and refractive index. Thus, the interference phase (phase shift experienced by the object beam), $\varphi(x, y)$ can be obtained using

$$
\varphi(x, y)=\frac{2 \pi}{\lambda}\left[n_{R B C}(x, y)-n_{\text {Plasma }}(x, y)\right] h(x, y)
$$

where $n(x, y)$ and $n_{o}(x, y)$ represent the refractive index of the cell and the surrounding medium respectively, and $h(x, y)$, represents the cell thickness at any given point. Thus, the refractive index can be obtained as long as the geometrical dimension of the object is known. Conversely, the thickness can be obtained as long as the refractive index of every pixel in the image is known.

This method of optical path measurement is very sensitive, allowing for precise micro-scale measurements of refractive indices in transparent mediums. Additionally, this method provides non-contact/non-destructive optical path change measurement, making it an ideal candidate for the measurement of the refractive index of living cells. A light beam with a wavelength of a few hundred nanometers passing through a cell a couple of microns in size, undergoes significant optical path (phase) change, due to the refractive index of the cell, changing the fringe pattern.

\subsection{Interference Phase Analysis}

The goal of interference phase analysis is the extraction of the required optical properties of the investigated object contained in the recorded interferogram. A typical interferogram (fringe pattern) can be seen in Figure 6-4. According to the literature, there are two common methods used for the analysis of interferograms: Phase Shifting Interferometry (PSI) and Fast Fourier Transform (FFT) [37,70,80,97]. The general form of the fringe pattern is obtained using

$$
g(x, y)=a(x, y)+b(x, y) \cos \left[2 \pi f_{o} x+\varphi(x, y)\right]
$$

where $\varphi(x, y)$, represents the phase containing the desired information, $a(x, y)$ and $b(x, y)$ represent the undesired illumination created by background and reflected light from the surroundings of the test object and $2 \pi f_{o} x$ represents high variation quantities introduced by the special carrier frequency $f_{o}$ caused by tilted wavefront to generate the fringes. The PSI method requires a minimum of three phase shifted interferograms to obtain the phase change and a simple algorithm to extract the phase information. Images produced by moving a component (such as a moving mirror mounted on translator) or tilting the reference mirror, causing a time shift to generally take on one of the following four discrete values typically found in the Michelson interferometer configuration: $(0, \pi / 2, \pi, 3 \pi / 2)$. The FFT method, on the other hand, 
which is commonly used in the MZI configuration, has a tilt set at zero. Eq.2 can then be simplified as

$$
g(x, y)=a(x, y)+b(x, y) \cos [\varphi(x, y)]
$$

Applying Euler's formula to Eq. 3 yields

$$
g(x, y)=a(x, y)+b(x, y) \frac{1}{2}\left[e^{i \varphi(x, y)}+e^{-i \varphi(x, y)}\right]
$$

Let

$$
c(x, y)=\frac{1}{2} b(x, y)\left[e^{i \varphi(x, y)}+e^{-i \varphi(x, y)}\right]
$$

Then we can write Eq.4 as follow

$$
g(x, y)=a(x, y)+c(x, y) e^{2 \pi i f_{o} x}+c^{*}(x, y) e^{-2 \pi i f_{o} x}
$$

Applying two-dimensional fast-Fourier-transform FFT algorithm to Eq. 6 yields

$$
G(f, v)=A(f, v)+C\left(f_{o}-f, v\right)+C^{*}\left(f_{o}+f, v\right)
$$

where $A(f, v)$ represents the transform of $a(x, y)$, and $C\left(f_{o}-f, v\right)$ and $C^{*}\left(f_{o}+f, v\right)$ are the positive and negative frequency spectra of the modulated carrier fringes.
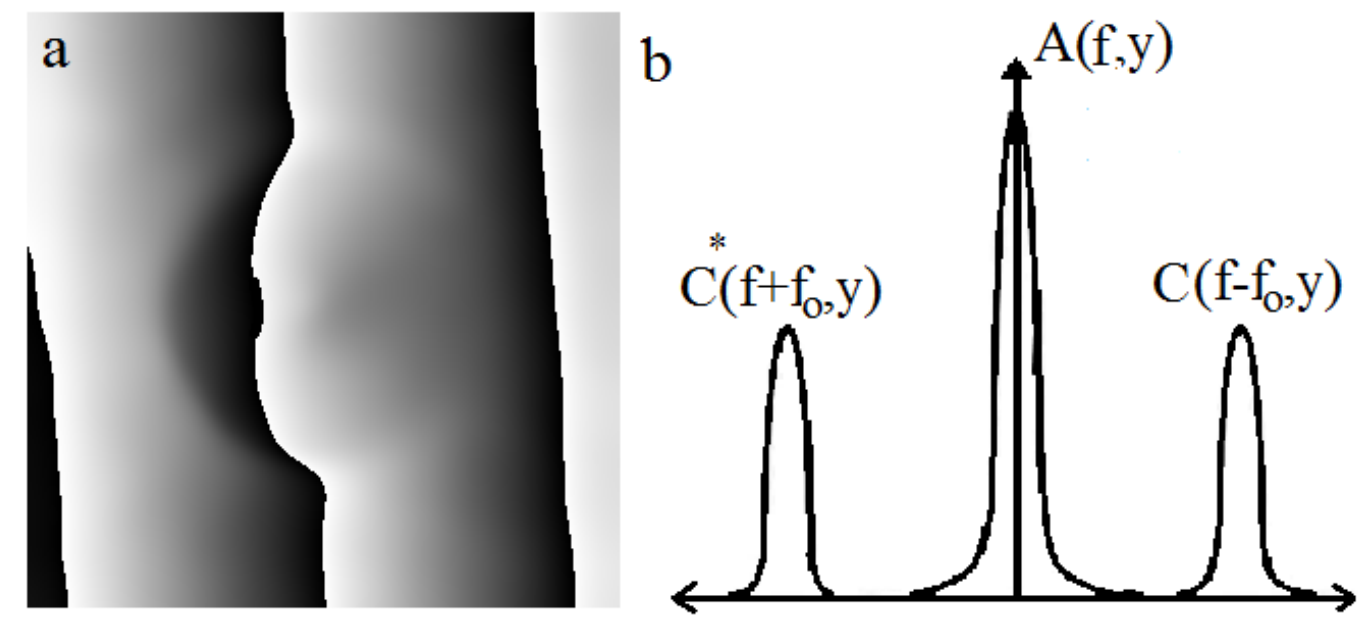

Figure 6-3: a- Phase shift image and b- Separated Fourier spectra signal of one fringe pattern.

As previously mentioned, the variation of $a(x, y), b(x, y)$, and $\varphi(x, y)$ are relatively small when compared to the carrier frequency $f_{o}$, The Fourier transform image of the interferogram, shown in Figure 1-8a, contains two spectres on both sides of the central frequency, and both $C\left(f_{o}+\right.$ $f, v)$ and $C^{*}\left(f_{o}-f, v\right)$ contain the same information. Through bandpass filtering in the special frequency domain, the unwanted background variation $A(f, y)$ and $C^{*}\left(f_{o}+f, v\right)$, can be 
removed. Applying an inverse FFT algorithm to $C\left(f_{o}+f, v\right)$ we obtain the complex value of $c(x, y)$. This can be expressed by the arctangent function used to compute the phase values written as

$$
\varphi(x, y)=\arctan \left(\frac{I_{m}[c(x, y)]}{R_{e}[c(x, y)]}\right)
$$

where the Fourier transform method is based on a least square linear fit of the harmonic and the interference pattern.

\subsubsection{Phase Unwrapping}

The phase $\varphi(x, y)$, obtained from the interferogram pattern, contains the desired information about the object under investigation, however, the information in these images appears in periodic values in the range of $(-\pi, \pi)$. The images in this stage are called "wrapped" phase images and they are represented as a measured function of

$$
\psi(i)=\varphi(i)+2 \pi k(i)
$$

where $k(i)$ represents an integer value that satisfies $-\pi<\psi \leq \pi$ and $i$ represents the array index. While the phase constantly increases, it remains bound by $-\pi$ and $\pi$. Figure 6-3a illustrates a typical example of the wrapped phase $\psi(x)$ and estimated unwrap phase of interest $\varphi(x)[148,149]$. Phase unwrapping is necessary in order to obtain $\varphi(i)$ from $\psi(i)$. This is can be done by adding $2 \pi$ to the function at each encountered phase discontinuity.

\subsubsection{Image Processing}

Interferograms captured with the digital camera can be processed to obtain information regarding specific phenomenon and one or several parameters of the object being recorded. A specific series of imaging processing steps need to be followed in order to enhance and extract information of interest from the images of the object under investigation. Matlab ${ }^{\circledR}$ has proven to be a powerful image processing and optical metrology tool in the field of digital image processing. Matlab ${ }^{\circledR}$ is a suitable platform for the implementation of image processing algorithms due to its ability to perform whole processing techniques and procedures when analysing images acquired by scanner, CCD cameras, etc. The software also provides a fast and flexible programming language for users constructing their own algorithms. In this section we provide fundamental information about image acquisition, filtering and processing. 


\subsubsection{Image Acquisition}

In this study, the image acquisition phase involved the process of recording the real object of interest in a digital, discrete video format using a CCD camera. Image processing was conducted in the Matlab ${ }^{\circledR}$ environment. In this stage, the "VideoReader" function of the CCD camera default factory software was used to obtain a series of interferogram images. The images were stored as frames. The function stores the object representation in a discrete $(m \times n)$ array, where the $m$ and $n$ indexes represent the spatial coordinates of the image.

\subsubsection{Threshold and Contrast Image}

Interferograms acquired in real conditions often contain background problems. Relevant foreground elements (such as RBCs) are sometimes mixed with background elements (such as the fringe pattern), causing a problem with the segmentation process required to draw the RBCs from the background. Algorithms such as equalization, binarization and thresholding can be used to enhance the appearance of the RBCs. The intensity of the images is adjusted to a specified range in order to increase the contrast in a low-contrast greyscale image and the data is then remapped to fill the entire intensity range $[0,255]$.

\subsubsection{Spatial Filtering}

Extracting the RBC cells from the recorded images is a challenging task since they are affected by high disturbance fringe lines. High-pass and low-pass filter are used in order to reduce the fringe noise and enhance the appearance of the RBC cells in the image. These filters were applied in both the frequency domain and the spatial domain. Spatial domain filtering activities are directly applied to the image (pixel to pixel) using mathematical functions known as convolution [150].

$$
f(x, y) * g(x, y)=\sum_{m=-\frac{M+1}{2}}^{\frac{M+1}{2}} \sum_{n=-\frac{N+1}{2}}^{\frac{N+1}{2}} f(m, n) g(x-m, y-n)
$$

where $f, g,(x, y),(m, n)$ and $M \times N$ represent the original image, the convolution mask or matrix, the coordinates of the original image, the coordinates of the convolution location, and the size of convolution mask, respectively. Eq.10 is applied by conducting homogeneous scanning 
with the convolution mask as opposed to the convolution of the entire image using the ' $f$ special' Matlab $^{\circledR}$ function.

\subsubsection{Low-Pass Filters}

Low-pass filters smooth the image by blurring the fringe edges and lowering the contrast. The primary feature of low-pass convolution masks is that all of the elements have positive values. Averaging, Gaussian and quadratic are some of the most commonly used low-pass filters. They are presented in a matrix form such as

$$
g(m, n)=\frac{1}{\sum_{m=1}^{M} \sum_{n=1}^{N} \omega_{m, n}}\left[\begin{array}{ccc}
\omega_{1,1} & \cdots & \omega_{1, N} \\
\vdots & \ddots & \vdots \\
\omega_{M, 1} & \cdots & \omega_{M, N}
\end{array}\right]
$$

where A, B and $\omega$ represent the amplitude, the width function factor and the weight function factor of the spatial filter, respectively. In order to determine the efficacy of the masks of equations (6) and (7), Magnitude Spectra (MS) are obtained in order to analyse the low frequencies permitted to pass by the filter and high frequency attenuation. This is expressed as

$$
\operatorname{MS}(\omega)=20 \log |\mathfrak{I}\{\mathrm{g}(\mathrm{m}, \mathrm{n})\}|
$$

where $\omega$ and $\mathfrak{I}$ represent the MS frequency component and the Fourier transform operator, respectively. Nine element masks were applied to the images generated by the 'Average' Matlab $^{\circledR}$ parameters using the ' $f$ special' function.

\subsubsection{High-pass Filters}

High-pass filters are another type of filter used in signal processing. High frequency components such as rapid tone changes and pronounced details are generally located in the image borders. High-pass filters are used for contrast enhancement and to highlight particular details. The high-boost, Gradient, Laplacian, Sobel, Prewitt and Canny are some of the most commonly used high-pass filters. The Canny filter provides the most powerful edge detection [150]. The Canny filter and nine element masks were applied to the images. 


\subsection{Results and Discussion}

Sampled of thinly smeared blood on a slide were prepared. The blood smears were freshly drawn from healthy donor using the finger prick method. In order to obtain a single layer with enough space between blood cells, whole blood droplets were sandwiched between cover slips by placing one drop at the end of one test slip and dispersing the blood using the spreader slide. The interferometric system was operated in the absence of the specimen under investigation in order to obtain clear contrast fringes. The specimen was then introduced into the optical path of the objective beam by placing it in front of the objective lens and adjusting the sample holder until both the blood cell image and clear interference fringes are produced. In order to ensure that the glass slide did not apply any pressure on the blood cells, a video was captured at 1 frame per second and revealed free movement of the blood cells, indicating that no pressure was applied. During the measurements, the temperature was monitored and maintained at $296 \pm 0.5 \mathrm{~K}$.

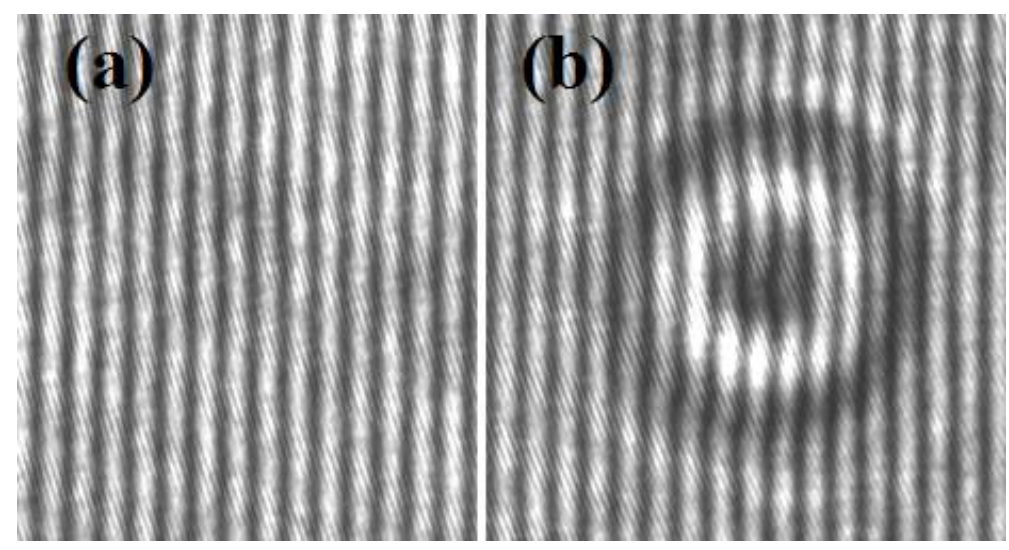

Figure 6-4: Interferogram a- in the absence of a blood sample and b- in the presence of the investigated RBC.

Figures 6-4 $(\mathrm{a}, \mathrm{b})$ shows quantitative phase maps in the absence and presence of RBCs, respectively. The interferograms captured by the CCD camera were then analysed to obtain the refractive index variation of the RBCs. The same interferogram were also used to estimate hemoglobin concentration. All data analysis was performed in the Matlab ${ }^{\circledR}$ environment. 


\subsubsection{Refractive Index}

A semi-automatic Matlab ${ }^{\circledR}$ routine based on the Fourier transform was developed by the authors in order to analyze the interferogram images and extract quantitative phase information. The routine begins by selecting a single RBC from different frames, avoiding cells that are turned onto their side or appear damaged.

Before any experimental measurements were conducted, the interferometric system was calibrated using an Improved Neubauer ${ }^{\circledR}$ Haemocytometer slide. A haemocytometer blood cell counting chamber with a sample depth of $0.1 \mathrm{~mm}$ and $0.0025 \mathrm{~m}^{-2}$ was used to convert the number of pixels into real world distance and the actual value found obtained was $4.18 \times 10^{-8} \mu \mathrm{m} / \mathrm{pixel}$. The user first selects two known points on the haemocytometer and the Matlab routine then converts them to the number of pixels corresponding to the system magnification. Hence, real cell morphology can be obtained for any selected cell.

In regions where no cells are present, refractive index variation should ideally correspond to the refractive index of the background (plasma) 1.34. The average standard deviation of the computed refractive index in the region where no cells are present is the axial resolution of the system, and in the present case, it was found to be $1.78 \times 10^{-4}$.
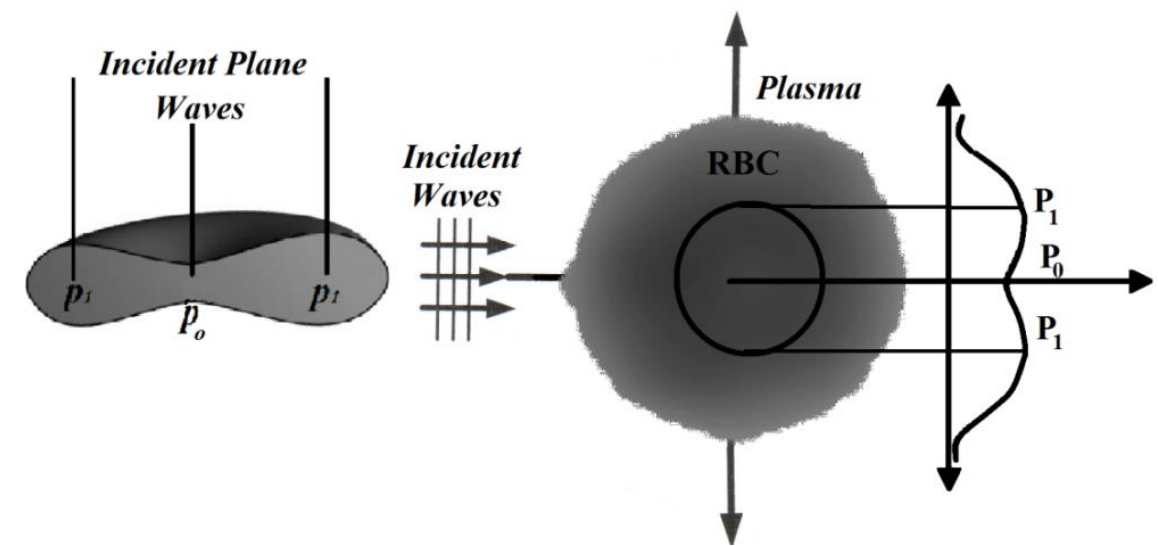

Figure 6-5: Schematic diagram of the incident waves on a single REB cell showing the refractive index measuring points.

In order to illustrate the capability of the proposed interferometric system and to provide detailed information about cell structure and dynamics, we analysed a few individual RBCs from different frames. The objective beam incident is perpendicular to the surface of the specimen under examination. Since the RBCs have irregular surfaces and varying thicknesses, only a few 
points were selected and considered in the refractive index calculations. Figure 6-5 (a,b) illustrates the cross-sectional image and the unwrap phase of the RBC and the selected measuring points, where the incident light waves at these points are perfectly perpendicular to the RBC. The phase change $\Delta \varphi$ in the interferogram, induced by the refractive index and thickness of the sample, were analysed based on the relation of $\Delta n=\frac{\lambda \Delta \varphi}{2 \pi \Delta h(x, y)}$, where $\Delta n=n_{c}-$ $n_{o}$ represents the refractive index difference between RBCs and the surrounding plasma, $h(x, y)$ represents the sample thickness, and $\lambda$ represents the light wavelength passing through the sample under investigation. The minimum and maximum thicknesses of the RBC used in the calculations $(1 \mu \mathrm{m}$ and $2 \mu \mathrm{m})$ were adopted from the literature (the average thickness of a healthy RBC is between $1.5 \mu \mathrm{m}$ and $2.2 \mu \mathrm{m}$ ) [112,151]. The phase shift calculations were selected for a few points at $\mathrm{P}_{\mathrm{o}}$ and circumference $\mathrm{P}_{1}$ and the average value of each set of data were used in the calculations. The comparison between the two set of values obtained from $\mathrm{P}_{\mathrm{o}}$ and $\mathrm{P}_{1}$ were $3.58 \times 10^{-3}$ confirming the homogenous hemoglobin distribution across the healthy RBCs. Figure 6-5 (b) is a schematic diagram of the incident waves on a single REB cell showing the refractive index measuring points. From the point of view of the refractive index, this variation confirms the homogenous nature of the $\mathrm{RBC}$. The average refractive index of the $\mathrm{RBC}$ was 1.42834 which appears to be in good agreement with the results obtained from the literature, with a standard deviation of $5.90 \times 10^{-3}[112,151]$. By confirming the homogenous nature of the refractive index in a healthy $\mathrm{RBC}$, the calculated refractive index was used to presents the thickness profile of any selected RBC. Since the refractive index was determined, the cell morphology can be obtained at any desired point. Figure 6-6 illustrates the thickness profile of a single RBC. The reconstructed thickness profile map was determined based on the prior knowledge of the refractive index of the cell.

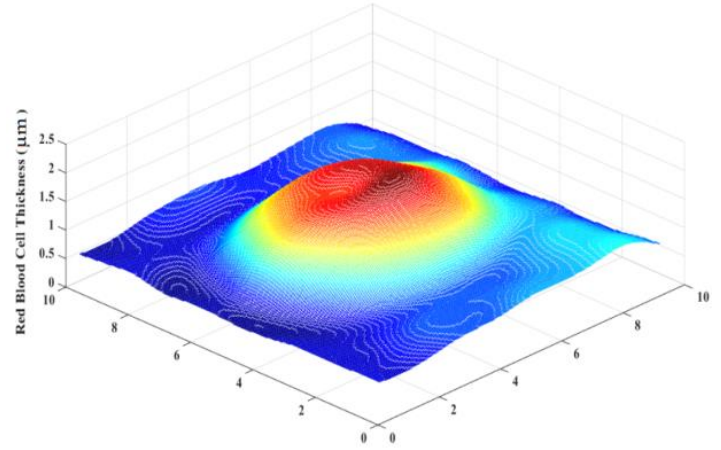

Figure 6-6: Three-dimensional thickness profile of single RBC. The phase information can be converted into the refractive index based on previous knowledge of certain properties. 


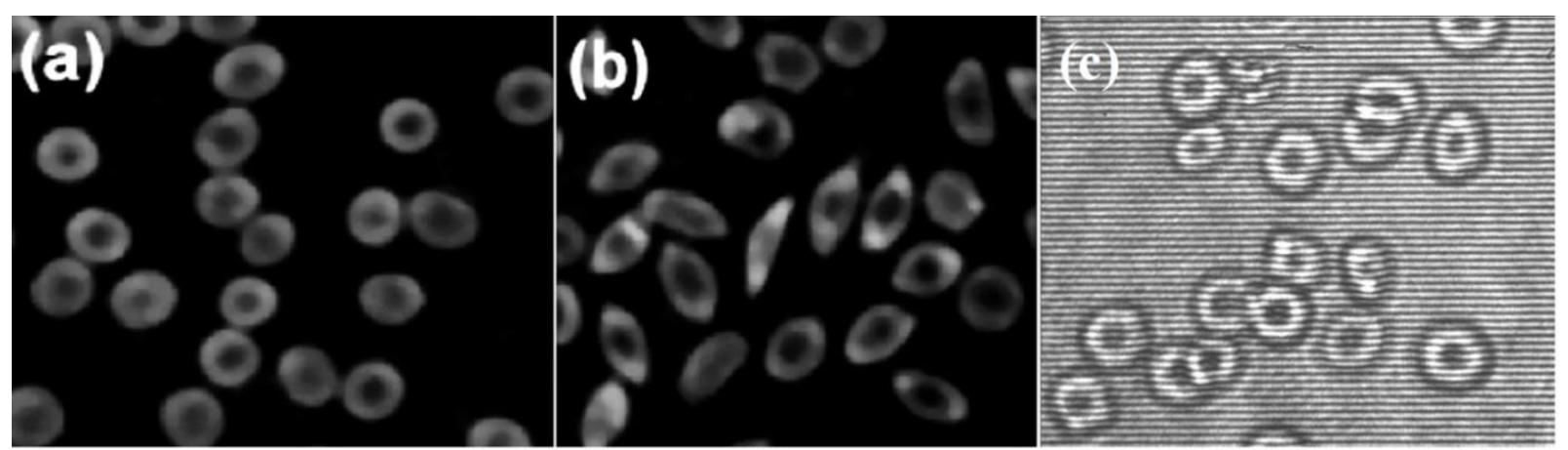

Figure 6-7: Microscopic images of a- healthy RBCs, b- Sickle RBCs obtained from the literature [116] and c- the RBC interferogram of a healthy donor obtained in this study.

Furthermore, all of the RBCs had a doughnut-shaped profile. It is thus possible to differentiate between healthy and unhealthy cells by comparing the shape of an unknown cell with the shape of a healthy cell, as shown in Figure 6-7. The experimental values are in good agreement with the values obtained in previous research $[112,151]$. Based on the previous discussion and data analysis, the health condition of the cells can be identified for any given sample by observing the refractive index, structural transformations and morphological changes of RBCs and their quality during the diagnostic and treatment stage. The quality of the transfusion blood can also be determined using imaging and by calculating the refractive index of the sample prior to storage and using it as reference to be compared with corresponding values at different storage periods.

\subsubsection{Hemoglobin Concentration}

Normal RBCs count levels are 2.6-4.8, 4.2-5.4 and 4.5-6.2 $\times 10^{6}$ cells $/ \mu$ in healthy children, women and men, respectively. In order to estimate the hemoglobin concentration, 10 diluted samples of 10-100\% were prepared using a pipette dropper and measured using a Denver Instrument ${ }^{\circledR}$ SI-234 balanced with a precision of 4 decimal places $\pm 1 \times 10^{-3} \mathrm{~g}$.

RBC (Hemoglobin) concentration (with respect to the volume of $6.861 \times 10^{-6} \mu \mathrm{l}$ ) was estimated using the recorded interferograms. Although counting RBCs in microscopic blood images is not a difficult task, it requires a lot of time and concentration, justifying the demand for automatic or semi-automatic blood cell counting techniques. While a number of valuable techniques have been proposed by researchers [152-155], there is a need for a more efficient, realistic and feasible approach. 
In order to calculate the total RBC count in the microscopic blood image, a custom Matlab ${ }^{\circledR}$ routine was developed to analyse the interferogram video sequence and calculate the number of RBCs in each frame. As previously mentioned, the blood cells in the interferograms experience high disturbance fringes, therefore, the images must undergo image processing in order to isolate and count the RBCs in the image.

This is done by converting the interferogram video file into multiple frames. The user then crops a certain area and applies a few image processing functions to enhance the appearance of the RBS in all frames. The Matlab ${ }^{\circledR}$ routine revealed relatively good agreement when compared to the manual blood cell count with an average accuracy of $87.58 \%$. This value was calculated based on the average hemoglobin concentration of $4.2-5.3 \times 10^{6}$ cells/ $\mu$ l of a healthy male donor. The results of $70 \%$ hemoglobin concentration along with image processing are shown in Figure 6-8(a-f).

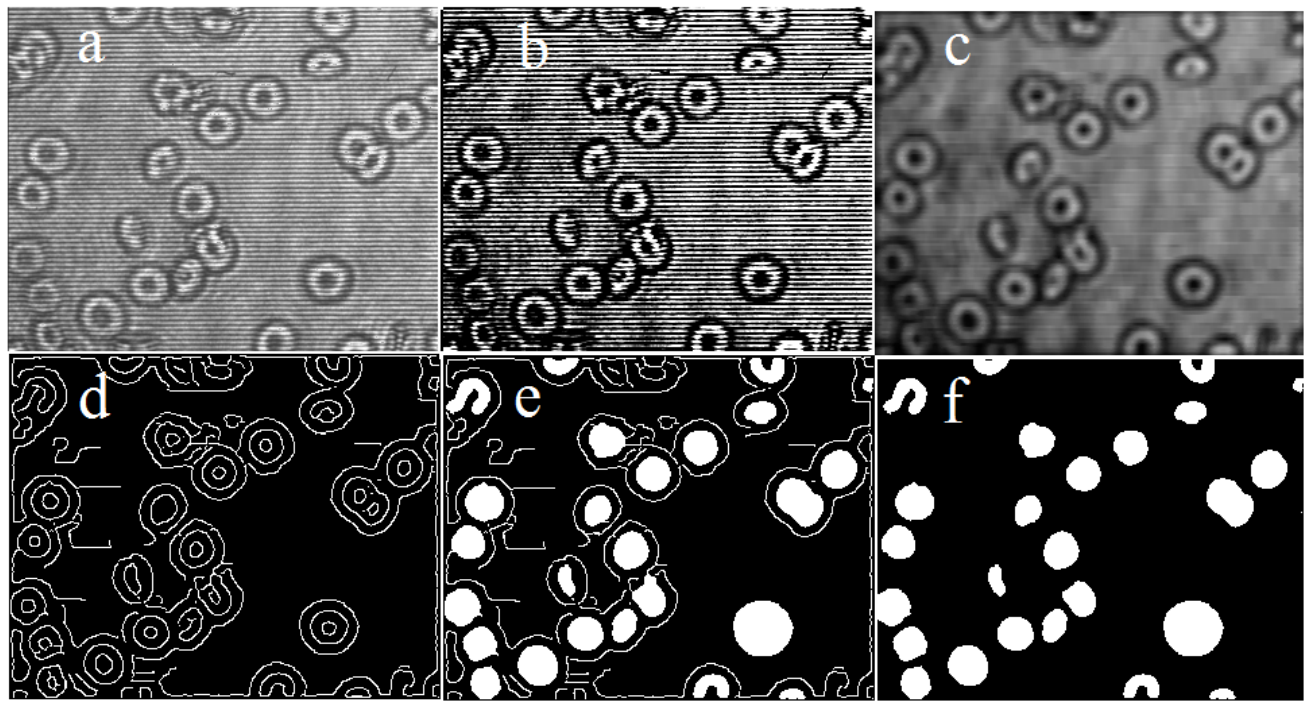

Figure 6-8: The image processing and cell segmentation process for one interferogram at $70 \%$ hemoglobin concentration, a. Original image, b. Adjusted threshold and contrast c. Low-pass "average filtered" image d. High-pass "Canny filtered" image e. Holes filling process f. Complete cell segmentation and cell counting.

The software automatically segments and calculates, for each frame, the number and surface area of the RBCs and records the information in an output table. Moreover, by adjusting the surface area limits we can isolate and count the number and types of cells in all captured frames.

Due to their biconcave shape, erythrocyte images have a much clearer centre than border. After each sub-image is filtered and adjusted using the relative Matlab ${ }^{\circledR}$ function, the $\mathrm{RBC}$ images appear as clear disk-shaped elements instead of unclear doughnut shaped elements. All of 
the clear disks and aggregate components of each frame of the image are then labelled and measured. In order to ensure the accuracy of the output results, a manual RBC count comparison was performed as shown in Table (6.1). The analysis results with different hemoglobin concentrations presented in in Figure 6-9, the error bars represent the standard deviation of automatically counted RBCs with the manually counted RBCs.

Table 6.1: Comparison results between the manual and the automated cells count.

\begin{tabular}{ccccc}
\hline \multirow{2}{*}{$\begin{array}{c}\text { Concentration } \\
\text { Hb g/l }\end{array}$} & \multicolumn{2}{c}{ No. of RBCs } & & \\
\cline { 2 - 3 } & $\begin{array}{c}\text { Manual } \\
\text { Count }\end{array}$ & $\begin{array}{c}\text { This } \\
\text { Study }\end{array}$ & Surface Area $\boldsymbol{\mu}^{2}$ & Efficiency $\%$ \\
\hline 355 & 36.5 & 32 & 24.490 & 87.671 \\
319.5 & 32 & 27 & 28.830 & 84.375 \\
284 & 27.5 & 25 & 25.165 & 90.909 \\
248.5 & 26 & 23 & 34.436 & 88.462 \\
213 & 20.5 & 17 & 31.113 & 82.927 \\
177.5 & 19 & 16 & 29.734 & 84.211 \\
142 & 13.5 & 11 & 27.155 & 81.481 \\
106.5 & 12 & 10 & 25.629 & 83.333 \\
71 & 8 & 7 & 28.744 & 87.500 \\
35.5 & 4 & 4 & 31.534 & 100.000 \\
\hline
\end{tabular}

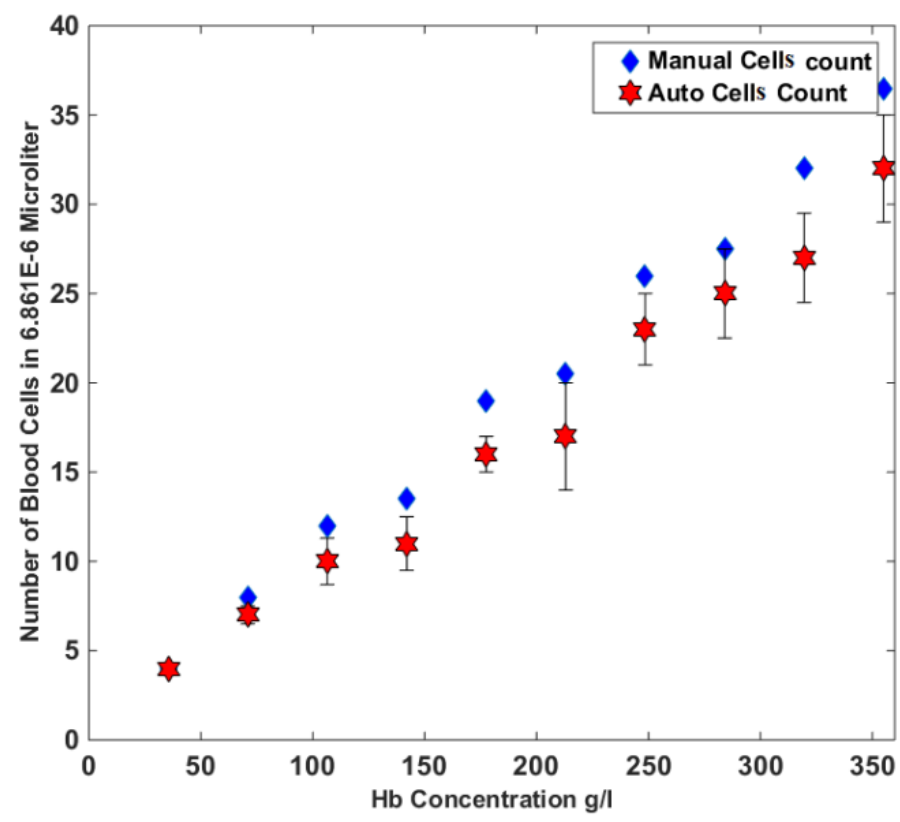

Figure 6-9: The results of the experiment and the manual cells count at various hemoglobin concentrations. 


\subsection{Conclusions}

This study provides a demonstration of the quantitative phase imaging and refractive index measurement of human RBCs using phase-shifting interference. The system was designed based on the Mach-Zehnder optical arrangement and proved successful at providing non-invasive quantitative phase images and optical properties of RBCs with nanoscale sensitivity using the Fourier transform method.

The phase-shifted interferograms are recorded using a CCD camera. Due to its non-invasive nature, the present technique is useful for the measurement of the morphological features and refractive index variation of living cells with high lateral and axial resolution. The system is quick to assemble and the optical components can be obtained at a low cost and may also be downsized as a standalone blood smear analyzer used as a point-of-care or bedside medical diagnostic micro-interferometric tool without relying on external measurements of hemoglobin concentrations or additional sample preparation such as stains or sample dilation. The instrument presented here requires significantly less computational work than digital holography and provides faster images than reported with other quantitative phase imaging techniques. The image analysis of the interferogram yielded useful information regarding RBC morphology and can be used to correlate the morphological profile of healthy/normal cells and unhealthy cells or those which imply blood cells disorders or diseases. The proposed method also complements the existing approach for automatic (or semi-automatic) blood cell counting. The same interferograms were used in the diagnosis of some selected diseases (e.g. anaemia or malaria) based on the deformations in the shape of RBCs. The average accuracy of the proposed system was $87.58 \%$. This demonstrated that the proposed system calculates results with a speed and accuracy approaching that of the manual method. RBC morphology and hemoglobin concentration are useful tools for the evaluation of the health condition of a patient.

The present technique has a few potential sources of error, including the poor contrast of the interference fringes and the pixel to real world distance conversion. This can be improved with the proper alignment of the interferometer, better selection of the dimmer filter, and by taking the average of multiple RBCs when conducting refractive index calculations. The present system is a potential technique for quantitative phase imaging and the high accuracy measurement of the refractive index of biological samples. 


\section{CHAPTER 7 CONCLUSIONS AND FUTURE WORKS}

\subsection{Work Conclusions}

Interference phenomenon occurs due to the superposition of two waves generated from the same coherence light source travelling different paths. Numerous interferometric techniques have been developed based on this phenomenon and are used in fields such as engineering metrology, fiber optics, astronomy and medicine. In engineering and medical science, interferometers are generally used to measure refractive index changes, cell pathology and shape irregularities. Optical interferometry is a non-contact method used for the imaging and measurement of the optical properties of small or large objects with nanoscale accuracy. This technique is non or minimally invasive and generally used for the imaging and measurement of the optical parameters of living cells.

The initial experimental setup was designed and developed based on Mach-Zehnder interferometry while employing a novel test cell holder that allowed for accurate heat distribution over the working cell. Precise experimental measurements of the refractive index of ternary hydrocarbon mixtures for the entire concentration range at various temperatures and wavelengths were proposed and verified using the Abbemat refractometer. The data analysis of the concentration, temperature and wavelength dependence of the refractive index revealed a linear dependence with both concentration and temperature while showing a non-linear dependence with wavelength. The experimental measurements presented in this part of the thesis led to the development of new empirical correlations which can be used to predict the refractive index of ternary mixtures as a function of concentration, temperature and wavelength with an average relative accuracy of $1.55 \times 10^{-3}$ using linear regression modeling based on the least squares approach and estimating the model parameters from the data.

The interferometric system was then modified to a MMZI system by allowing a precise direct laser beam passing through a specimen and magnifying the penetrating light using a 20X objective lens to study the blood analysis. Blood analysis is a routine procedure used in medical diagnostics to confirm a patient's condition and identify possible diseases. It is thus essential for this procedure to be available to individuals at no cost or at an affordable cost. Unfortunately, the

clinical devices are expensive and thus unaffordable to the average person in developing countries. The availability of compact clinical devices which can provide imaging and optical 
measurement of human blood is fairly limited. The primary achievement of this research project was the development of an interferometric system based on Mach-Zehnder interferometry. The system provided high quality images of blood cell morphology and accurate measurement of optical properties at a low cost with minimal effort.

Since the refractive index is a fundamental optical property, it is often used to identify the condition of blood cells and confirm their health status. This is a difficult task due to the inherent nature of blood and the large variation in the values reported in the literature. In this study, a new empirical model was developed in order to predict the refractive index of human blood mimicking phantom using the Abbemat refractometer. This substance shows better stability compared to human blood in experimental measurement conditions. The model was developed based on the well-known linear regression method. In this method, model parameters can be obtained by solving an optimization problem. The results of the comparison confirmed the system's ability to predict the refractive index values of human blood as a function of concentration, temperature and wavelength with an acceptable clinical accuracy of $2.33 \times 10^{-3}$.

The accuracy of the interferometric system was verified using the data obtained using the refractometer and the data obtained from the literature. The system was then used to obtain images and refractive index measurements of blood smear samples obtained from healthy donors using the finger prick method. The samples obtained using this method was sufficient to perform all of the required measurements. The values obtained using the interferogram captured by the CCD camera were then analysed using the Fourier transform method. This method was adopted because it only requires a single image in order to obtain the phase shift information.

By visualizing single images captured by the CCD, blood cells can be identified with minimal effort and without the need for extra sample preparation such as cell separations and blood stains. Cell abnormalities can also be observed and diagnosed by looking at the image, specifically, changes in size, shape or coating of the RBCs.

Using a computer routine developed in the Matlab environment, the user can obtain the refractive index and cell morphology of a single, manually selected cell. In order to estimate the hematocrit values of the blood, the computer routine uses a series of images to segment and count the number of RBCs per volume unit. The results of the analysis revealed the program's ability to determine the refractive index and/or haematocrit values of human blood with appropriate clinical accuracy. 


\subsection{Contributions and Future Work}

Interferometric techniques are very powerful and can be used for various purposes in the field of engineering and science. These methods are capable of providing accurate phase measurements with nanoscale accuracy. Because of its non-contact and minimally invasive nature, interferometry is recommended for the study of live cells.

This dissertation has developed and setup an interferometric system and provided precise measurements of the refractive index of hydrocarbons and biological samples, whereas the major contributions are:

- The initial experimental setup was designed and developed based on Mach-Zehnder interferometry and employed a novel test cell holder that allowed for accurate heat distribution over the working cell.

- Provide precise values of the contrast factors of two different ternary systems of THN, IBB and $n C_{12}$ of the following concentration targets: molar fraction of (0.501-0.110$0.389)$ and (0.441-0.217-0.342) that's missing in the literature.

- The experimental measurements presented new empirical correlations can be used to calculate the refractive index of the study ternary hydrocarbon mixture with average relative accuracy of $1.55 \times 10^{-3}$.

- Developed a model that can be used to estimate the refractive index of human hemoglobin solutions as a function of concentration, temperature and wavelength at physiological level and an acceptable clinical accuracy of $2.33 \times 10^{-3}$.

- The system provided high quality images of blood cell morphology and accurate measurement of optical properties at a low cost with minimal effort.

Few areas in this dissertation are not addressed or covered and are suggested for the direction of future studies:

I. The contrast factors of the ternary hydrocarbon mixtures THN, IBB and $n \mathrm{C}_{12}$ are essential parameters in the study of thermal diffusion, an area of great interest to CSA and ISS. There is, however, a limited amount of research on these factors available in the literature. It is thus of great interest to this field of study to examine all of the ternary mixtures missing from the literature. 
II. All of our measurements were performed using a single wavelength of $632.9 \mathrm{~nm}$. There are many advantages to reproducing the same measurements with two wavelengths:

a. Doing so allows us to compare the results in order to confirm validity and a higher accuracy.

b. The refractive indices of the RBCs were obtained using published information regarding the geometry and thickness of RBCs. Measuring the same blood smear sample for two wavelengths would allow for the full measurement of the refractive index and thickness of the blood cells simultaneously without any previous knowledge of either property.

III. This study examined the refractive index and the morphological profile of RBCs, literature survey revealed that storage procedures and storage time cause morphological changes in RBCs during blood bank storage. We thus recommend studying the morphology images of RBCs and their refractive index to determine the blood transfusion quality at various stages of storage under blood bank conditions.

IV. The system magnification used in this study was $20 \mathrm{X}$ which was established using one type of objective lens. The study highly recommends using higher power objective lenses in order to study the samples on a more detail level, possibly even at the chromosomic level.

V. Based on our extensive literature survey of the pre-storage procedure for RBCs, the RBCs undergo a certain level of radiation in order to kill the WBCs that have made it past the filtration process. The study recommends that the RBCs be studied before and after the radiation process. 


\section{Appendices}

Appendix 3.1: Refractive indices and temperature coefficients of THN, IBB, and $n C_{12}$ at temperatures of $T=$ (293.15 to 318.15) $\mathrm{K}$ wavelength of $632.9 \mathrm{~nm}$ at atmospheric pressure $\mathrm{P}=0.1 \mathrm{MPa}^{\mathrm{a}}$.

\begin{tabular}{|c|c|c|c|c|c|c|c|c|c|}
\hline \multicolumn{3}{|c|}{ Mole Fraction } & \multicolumn{6}{|c|}{ Wavelength $\lambda=632.9 \quad \mathrm{~nm}$} & \multirow{2}{*}{$(d n / d T)$} \\
\hline THN & IBB & $n \mathrm{C}_{12}$ & 293.15 & 298.15 & 303.15 & 308.15 & 313.15 & 318.15 & \\
\hline 0 & 0 & 1 & & & & & & & $-4.34 \mathrm{E}-04$ \\
\hline 0 & 1 & 0 & 1.48377 & 1.48132 & 1.47888 & 1.47643 & 1.47397 & 1.47151 & -4 90F_-04 \\
\hline 1 & 0 & 0 & 1.53808 & 1.53565 & 1.53321 & & 1.52844 & 29 & \\
\hline \multicolumn{10}{|c|}{ Binary mixtures of $\left(\mathrm{n}_{\mathrm{IBB}}\right) n \mathrm{C}_{12}$} \\
\hline 0 & 0.9014 & 0.0986 & 1.47617 & 1.47371 & 1.47135 & 1.46871 & 1.46652 & 1.46372 & $-4.94 \mathrm{E}-04$ \\
\hline 0 & 0.8024 & 0.1976 & 1.46884 & 1.46646 & 1.46405 & 1.46165 & 1.45924 & 1.45679 & $-4.82 \mathrm{E}-04$ \\
\hline 0 & 0.7032 & 0.2968 & 1.46196 & 1.45965 & 1.45721 & 1.45488 & 1.45247 & 1.45008 & $-4.76 \mathrm{E}-04$ \\
\hline 0 & 0.6036 & 0.3964 & 1.45524 & 1.45293 & 1.45059 & 1.44827 & 1.4459 & 1.44352 & $-4.69 \mathrm{E}-04$ \\
\hline 0 & 0.5038 & 0.4962 & 1.44884 & 1.44642 & 1.44427 & 1.44178 & 1.43965 & 1.4371 & $-4.66 \mathrm{E}-04$ \\
\hline 0 & 0.4036 & 0.5964 & 1.44255 & 1.44035 & 1.43796 & 1.4358 & 1.43336 & 1.43114 & $-4.58 \mathrm{E}-04$ \\
\hline 0 & 0.3032 & 0.6968 & 1.4368 & 1.43449 & 1.43232 & 1.42979 & 1.42752 & 1.425 & $-4.71 \mathrm{E}-04$ \\
\hline 0 & 0.2024 & 0.7976 & 1.43094 & 1.42885 & 1.42643 & 1.42433 & 1.42191 & 1.41993 & $-4.46 \mathrm{E}-04$ \\
\hline 0 & 0.1014 & 0.8986 & 1.42554 & 1.42337 & 1.42115 & 1.41894 & 1.41677 & 1.41454 & $-4.40 \mathrm{E}-04$ \\
\hline \multicolumn{10}{|c|}{ Binary mixtures of $\left(\mathrm{n}_{\mathrm{THN}}\right) n \mathrm{C}_{12}$} \\
\hline 0.1014 & 0 & 0.8986 & 1.42949 & 1.42729 & 1.42505 & 1.42297 & 1.42066 & 1.41859 & $-4.37 \mathrm{E}-04$ \\
\hline 0.2024 & 0 & 0.7976 & 1.43917 & 1.43699 & 1.4347 & 1.43234 & 1.43026 & 1.42777 & $-4.55 \mathrm{E}-04$ \\
\hline 0.3032 & 0 & 0.6968 & 1.44918 & 1.44692 & 1.44438 & 1.44222 & 1.43979 & 1.43785 & $-4.58 \mathrm{E}-04$ \\
\hline 0.4036 & 0 & 0.5964 & 1.45999 & 1.45768 & 1.45549 & 1.45318 & 1.45075 & 1.44826 & $-4.67 \mathrm{E}-04$ \\
\hline 0.5038 & 0 & 0.4962 & 1.47113 & 1.4689 & 1.46668 & 1.46436 & 1.46185 & 1.45981 & $-4.58 \mathrm{E}-04$ \\
\hline 0.6036 & 0 & 0.3964 & 1.48318 & 1.4807 & 1.47855 & 1.4762 & 1.47398 & 1.47165 & $-4.58 \mathrm{E}-04$ \\
\hline 0.7032 & 0 & 0.2968 & 1.49553 & 1.4932 & 1.49077 & 1.48855 & 1.48614 & 1.48389 & $-4.66 \mathrm{E}-04$ \\
\hline 0.8024 & 0 & 0.1976 & 1.50887 & 1.50652 & 1.50415 & 1.50145 & 1.49928 & 1.49667 & $-4.88 \mathrm{E}-04$ \\
\hline 0.9014 & 0 & 0.0986 & 1.52288 & 1.52049 & 1.51798 & 1.51565 & 1.51325 & 1.51123 & $-4.70 \mathrm{E}-04$ \\
\hline \multicolumn{10}{|c|}{ Binary mixtures of $\left(n_{T H N}\right)$ IBB } \\
\hline 0.1014 & 0.8986 & 0 & 1.4889 & 1.48633 & 1.48406 & 1.48142 & 1.47918 & 1.47656 & $-4.90 \mathrm{E}-04$ \\
\hline 0.2024 & 0.7976 & 0 & 1.49381 & 1.49149 & 1.48893 & 1.48659 & 1.48409 & 1.48179 & $-4.84 \mathrm{E}-04$ \\
\hline 0.3032 & 0.6968 & 0 & 1.49922 & 1.49681 & 1.49439 & 1.4917 & 1.48957 & 1.4869 & $-4.91 \mathrm{E}-04$ \\
\hline 0.4036 & 0.5964 & 0 & 1.50445 & 1.50206 & 1.49932 & 1.49701 & 1.49422 & 1.49181 & $-5.09 \mathrm{E}-04$ \\
\hline 0.5038 & 0.4962 & 0 & 1.50978 & 1.50737 & 1.50493 & 1.50224 & 1.49959 & 1.49721 & $-5.08 \mathrm{E}-0$ \\
\hline 0.6036 & 0.3964 & 0 & 1.51528 & 1.51298 & 1.51036 & 1.50811 & 1.50542 & 1.50325 & $-4.86 \mathrm{E}-04$ \\
\hline 0.7032 & 0.2968 & 0 & 1.52085 & 1.51853 & 1.51604 & 1.51368 & 1.51132 & 1.50887 & $-4.79 E-04$ \\
\hline
\end{tabular}




\begin{tabular}{|c|c|c|c|c|c|c|c|c|c|}
\hline 3024 & 5 & 0 & & & & & & & \\
\hline 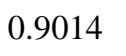 & 0986 & 0 & 53226 & 1.52981 & 1.52748 & 1.52477 & 52248 & 51989 & $05 \mathrm{E}$ \\
\hline \multicolumn{10}{|c|}{ Ternary mixtures of THN|IBB $n C_{12}$} \\
\hline 0.122 & 0.1202 & .7578 & 1.43514 & 1.43291 & 1.43072 & 1.42852 & & .42411 & $-4.42 \mathrm{E}-04$ \\
\hline 0.119 & .2344 & 6466 & 44088 & .43866 & 1.43641 & .43414 & 43192 & .42964 & $-4.50 \mathrm{E}-04$ \\
\hline 2376 & 0.117 & 454 & & & & & & & 50504 \\
\hline & & & & & & & & & \\
\hline 3472 & 114 & 5389 & 45561 & & 1.45103 & .44874 & 44651 & 44442 & \\
\hline & & & & & & & & & \\
\hline & & & & & & & & & \\
\hline 0.339 & 0.2226 & 4385 & .46208 & & & & & & \\
\hline & & & & & & & & & \\
\hline & 5454 & 8 & & & & & & & \\
\hline 3311 & 0.3262 & 3427 & & & & & & & \\
\hline & & 3416 & & & & & & & \\
\hline & 0.6397 & & & & & & & & \\
\hline 2161 & & & & & & & & & \\
\hline & & & & & & & & & \\
\hline 4309 & 0.3183 & .2508 & & & & & & & \\
\hline 5277 & 0.2119 & 0.2504 & & & & & & & \\
\hline 8442 & 1058 & & & & & & & & \\
\hline & & & & & & & & & \\
\hline & & & & & & & & & \\
\hline 5259 & 0.3108 & 1633 & & & & & & & \\
\hline 734 & 1033 & 1628 & & & & & & & \\
\hline & & & & & & & & & \\
\hline 2068 & 0.7129 & .0803 & & 369 & & & & & \\
\hline 3097 & 0.6101 & 0.0801 & & & & & & & \\
\hline 4123 & & & & & & & & & \\
\hline 0.5146 & 0.4055 & 0.0799 & 129 & & 607 & 1.49374 & & & \\
\hline 6166 & 0.3037 & 0798 & .50671 & 1.50421 & 1.50178 & 1.4994 & 1.49709 & 1.49472 & \\
\hline 7182 & 0.2021 & 0796 & & & & & & & \\
\hline 0.8196 & 0.1009 & & & & & & & & \\
\hline 0.2318 & 0.2284 & 0.5398 & 1.45113 & 1.44883 & 1.44649 & 1.44417 & 1.44203 & 1.43975 & $-4.55 \mathrm{E}-($ \\
\hline 0301 & 0.2069 & & .49803 & 1.49552 & 1.49303 & 1.49063 & 1.48821 & 1.48584 & $-4.87 \mathrm{E}-04$ \\
\hline 0.2114 & 0.6246 & & 1.47836 & & & 1.47124 & & 1.46634 & \\
\hline & 0.4356 & & & 1.46183 & 1.45959 & 1.45702 & 1.45475 & 1.45227 & $-4.19 \mathrm{E}-04$ \\
\hline
\end{tabular}




$$
\begin{array}{llllllllll}
0.4408 & 0.2171 & 0.3421 & 1.4734 & 1.47068 & 1.46884 & 1.46628 & 1.46396 & 1.46153 & -4.69 \mathrm{E}-04 \\
0.4214 & 0.4151 & 0.1635 & 1.48828 & 1.48592 & 1.4836 & 1.48126 & 1.47894 & 1.47658 & -4.67 \mathrm{E}-04
\end{array}
$$

a The reported average expanded uncertainty $U=1.197 \times 10^{-3}$ was calculated based on the coverage factor $k=2$, providing a level of confidence of approximately $95 \%$.

The average standard deviation comparisons of the temperature coefficient of the refractive index with available literature [15] were presented in table 3.7.

Appendix 3.2: Refractive indices and temperature coefficients of THN, IBB, and $n C_{12}$ at temperatures of T=

\begin{tabular}{|c|c|c|c|c|c|c|c|c|c|}
\hline \multicolumn{3}{|c|}{ Mole Fraction } & \multicolumn{6}{|c|}{ Wavelength $\lambda=589.3 \mathrm{~nm}$} & \multirow{2}{*}{$(\mathrm{dn} / \mathrm{dT})$} \\
\hline THN & IBB & $n \mathrm{C}_{12}$ & 293.15 & 298.15 & 303.15 & 308.15 & 313.15 & 318.15 & \\
\hline 1 & $\overline{0}$ & 0 & 1.5412 & 1.53875 & 1.53628 & 1.53389 & 1.53148 & 1.52912 & $-4.83 \mathrm{E}-04$ \\
\hline 0 & 1 & 0 & 1.48654 & 1.48406 & 1.4815 & 1.47904 & 1.47657 & 1.47409 & $-4.98 \mathrm{E}-04$ \\
\hline 0 & 0 & 1 & 1.42171 & 1.41957 & 1.41742 & & 1.41309 & 1.41093 & \\
\hline \multicolumn{10}{|c|}{ Binary mixtures of $\left(\mathrm{n}_{\mathrm{IBB}}\right) n \mathrm{C}_{12}$} \\
\hline 0 & 0.9014 & 0.0986 & 1.47869 & 1.47611 & 1.47362 & 1.47126 & 1.46878 & 1.46615 & $-4.97 \mathrm{E}-04$ \\
\hline 0 & 0.8024 & 0.1976 & 1.47122 & 1.46882 & 1.46639 & 1.46398 & 1.46154 & 1.4591 & $-4.85 \mathrm{E}-04$ \\
\hline 0 & 0.7032 & 0.2968 & 1.46421 & 1.46188 & 1.45946 & 1.45708 & 1.45466 & 1.45224 & $-4.79 \mathrm{E}-04$ \\
\hline 0 & 0.6036 & 0.3964 & 1.45746 & 1.45504 & 1.45269 & 1.45034 & 1.44797 & 1.44556 & $-4.75 \mathrm{E}-04$ \\
\hline 0 & 0.5038 & 0.4962 & 1.45083 & 1.44841 & 1.44611 & 1.44375 & 1.44159 & 1.43898 & $-4.69 \mathrm{E}-04$ \\
\hline 0 & 0.4036 & 0.5964 & 1.44443 & 1.44221 & & 1.43762 & & 1.43297 & $-4.60 \mathrm{E}-04$ \\
\hline 0 & 0.3032 & 0.6968 & 1.43856 & 1.43623 & 1.43406 & 1.43152 & 1.42938 & 1.42669 & $-4.71 \mathrm{E}-04$ \\
\hline 0 & 0.2024 & 0.7976 & 1.4326 & 1.43032 & 1.42804 & 1.42581 & 1.42345 & 1.42122 & $-4.56 \mathrm{E}-04$ \\
\hline 0 & 0.1014 & & 1.42708 & 1.4249 & 1.42267 & & 1.41826 & 1.41596 & $-4.44 \mathrm{E}-04$ \\
\hline \multicolumn{10}{|c|}{ Binary mixtures of $\left(\mathrm{n}_{\mathrm{THN}}\right) n \mathrm{C}_{12}$} \\
\hline 0.1014 & 0 & 0.8986 & 1.43105 & 1.42884 & 1.42659 & 1.4245 & 1.42227 & 1.42009 & $-4.38 \mathrm{E}-04$ \\
\hline 0.2024 & 0 & 0.7976 & 1.44086 & 1.43868 & 1.43636 & 1.434 & 1.43177 & 1.42933 & $-4.61 \mathrm{E}-04$ \\
\hline 0.3032 & 0 & 0.6968 & 1.45101 & 1.44896 & 1.44635 & 1.44408 & 1.44157 & 1.43955 & $-4.67 \mathrm{E}-04$ \\
\hline 0.4036 & 0 & 0.5964 & 1.46197 & 1.45965 & 1.45733 & 1.45488 & 1.45242 & 1.44985 & $-4.84 \mathrm{E}-04$ \\
\hline 0.5038 & 0 & 0.4962 & 1.47329 & 1.47078 & 1.46841 & 1.46618 & 1.46372 & 1.46145 & $-4.72 \mathrm{E}-04$ \\
\hline 0.6036 & 0 & 0.3964 & 1.48552 & 1.48302 & 1.48085 & 1.47848 & 1.47625 & 1.47389 & $-4.62 \mathrm{E}-04$ \\
\hline 0.7032 & 0 & 0.2968 & 1.49803 & 1.4955 & 1.49318 & 1.49085 & 1.48857 & 1.48615 & $-4.72 \mathrm{E}-04$ \\
\hline 0.8024 & 0 & 0.1976 & 1.51156 & 1.50927 & 1.50685 & 1.50425 & 1.50166 & 1.49906 & $-5.02 \mathrm{E}-04$ \\
\hline 0.9014 & 0 & 0.0986 & 1.52591 & 1.52353 & 1.52095 & 1.51872 & 1.51623 & 1.51403 & $-4.77 \mathrm{E}-04$ \\
\hline \multicolumn{10}{|c|}{ Binary mixtures of $\left(n_{\mathrm{THN}}\right)$ IBB } \\
\hline 0.1014 & 0.8986 & 0 & 1.49141 & 1.48927 & 1.48673 & 1.48428 & 1.48198 & 1.47955 & $-4.78 \mathrm{E}-04$ \\
\hline 0.2024 & 0.7976 & 0 & 1.49657 & 1.49432 & 1.49163 & 1.48939 & 1.48705 & 1.48455 & $-4.81 \mathrm{E}-04$ \\
\hline
\end{tabular}
(293.15 to 318.15$) \mathrm{K}$ wavelength of $589.2 \mathrm{~nm}$ at atmospheric pressure $\mathrm{P}=0.1 \mathrm{MPa}^{\mathrm{a}}$. 


\begin{tabular}{|c|c|c|c|c|c|c|c|c|c|}
\hline & & 0 & & & & & & & \\
\hline 4036 & .5964 & 0 & 50701 & .50446 & & & .49702 & .49455 & 4.98E-04 \\
\hline 5038 & 4962 & 0 & 51266 & .51023 & .50776 & .50515 & 1.50259 & 1.49987 & $-5.11 \mathrm{E}-04$ \\
\hline 5036 & & & & & & & & & $-4.87 \mathrm{E}-04$ \\
\hline & & & & & & & & & \\
\hline 8024 & 1976 & 0 & .52961 & 1.52722 & 1.52478 & & 1.52003 & 1.51758 & $-4.81 \mathrm{E}-04$ \\
\hline & & 0 & & & & & & .52287 & $-4.95 \mathrm{E}-04$ \\
\hline \multicolumn{9}{|c|}{ Ternary mixtures of $\mathrm{THN}|\mathrm{IBB}| n \mathrm{C}_{12}$} & \\
\hline 122 & 0.1202 & .7578 & 1.4368 & 1.43458 & 1.43237 & 1.43017 & & 1.42572 & $-4.43 E-04$ \\
\hline & 0.2344 & & & & & & & 1.43135 & \\
\hline 2376 & 0.1 & & & & & & & & \\
\hline 161 & 0.3431 & 5408 & 1.44895 & 1.44674 & 1.44437 & & 1.43979 & 1.43758 & -4 57F- 04 \\
\hline & & & & & & & & & \\
\hline 134 & 0.4467 & & & & & & & & $0 \mathrm{E}-04$ \\
\hline 2264 & 0.3344 & 4392 & 1.4596 & 1.45726 & 1.45495 & & 1.45031 & & $-4.60 \mathrm{E}-04$ \\
\hline ה קנJ & 0.2226 & & & & & & & & \\
\hline 4512 & 0.1111 & 4377 & 1.46872 & 1.46647 & & & 1.45943 & .45712 & $-4.66 \mathrm{E}-04$ \\
\hline 107 & 0.5454 & - & & & & & & & \\
\hline & 3262 & & & & & & & & \\
\hline 5501 & 0.1084 & 3416 & 1.4805 & 1.47798 & 1.47568 & 1.47314 & 1.47067 & .46799 & $-4.97 \mathrm{E}-04$ \\
\hline 1082 & 0.6397 & 252 & 1.46895 & 1.46652 & 1.46408 & 1.46154 & 1.4593 & & E-04 \\
\hline 161 & .5322 & 2516 & 361 & & & & & & \\
\hline 3237 & 0.4251 & & & & & & & & \\
\hline 4309 & & & & & & & & & \\
\hline 5377 & 0.2119 & 2504 & 1.48807 & & & & 7866 & 7634 & $E-04$ \\
\hline 6442 & 0.1058 & & & & & & & & \\
\hline 1059 & 0.7298 & 3 & 1.47606 & & & & & & \\
\hline 3166 & 0.5197 & 1638 & 1.4859 & 1.48323 & 1.48076 & 7866 & 1.47622 & 1.47392 & $-4.74 \mathrm{E}-04$ \\
\hline & 0.3108 & 1633 & 1.49594 & & & & & & $-4.89 \mathrm{E}-04$ \\
\hline 0.734 & 0.1033 & 1628 & 1.50621 & & & & & & \\
\hline .1036 & 0.8161 & 0.0804 & 1.48355 & 1.48105 & 1.47858 & 1.47614 & 1.47358 & 1.47103 & $-5.00 \mathrm{E}-04$ \\
\hline 2068 & & 0.0803 & & & & & & & $-5.02 \mathrm{E}-04$ \\
\hline 3097 & 0.6101 & 0.0801 & 1.49364 & & 1.4 & & & & 1E-04 \\
\hline 0.4123 & 0.5077 & & & & & 1.49085 & 1.48835 & 1.48568 & -5. \\
\hline 0.5146 & 0.4055 & 0.0799 & 1.50401 & & 1.49888 & 1.49635 & 1.49384 & 1.49154 & $-5.01 \mathrm{E}-04$ \\
\hline 0.6166 & 0.3037 & 0.0798 & 1.50947 & 1.50705 & 1.50472 & 1.50228 & 1.50003 & 1.49755 & $-4.75 \mathrm{E}-04$ \\
\hline 0.7182 & 0.2021 & 0.0796 & 1.51462 & 1.51247 & 1.50989 & 1.50767 & 1.50495 & 1.50254 & $-4.87 \mathrm{E}-04$ \\
\hline
\end{tabular}




$\begin{array}{llllllllll}0.8196 & 0.1009 & 0.0795 & 1.52026 & 1.51796 & 1.51549 & 1.51317 & 1.51082 & 1.50844 & -4.73 \mathrm{E}-04 \\ 0.2318 & 0.2284 & 0.5398 & 1.45306 & 1.45075 & 1.44858 & 1.44624 & 1.44393 & 1.44179 & -4.52 \mathrm{E}-04 \\ 0.6301 & 0.2069 & 0.163 & 1.50063 & 1.4981 & 1.49563 & 1.49318 & 1.4907 & 1.48832 & -4.93 \mathrm{E}-04 \\ 0.2114 & 0.6246 & 0.1641 & 1.48081 & 1.47838 & 1.47599 & 1.47368 & 1.47121 & 1.46872 & -4.82 \mathrm{E}-04 \\ 0.2211 & 0.4356 & 0.3433 & 1.46628 & 1.464 & 1.46173 & 1.45916 & 1.45687 & 1.45448 & -4.74 \mathrm{E}-04 \\ 0.4408 & 0.2171 & 0.3421 & 1.47565 & 1.47347 & 1.47107 & 1.46877 & 1.46648 & 1.46397 & -4.67 \mathrm{E}-04 \\ 0.4214 & 0.4151 & 0.1635 & 1.49081 & 1.48845 & 1.48611 & 1.48378 & 1.4814 & 1.47925 & -4.64 \mathrm{E}-04\end{array}$

${ }^{a}$ The reported average expanded uncertainty $U=1.197 \times 10^{-3}$ was calculated based on the coverage factor $k=2$, providing a level of confidence of approximately $95 \%$.

The average standard deviation comparisons of the temperature coefficient of the refractive index with available literature [15] were presented in table 3.7 .

Appendex 3.3: Refractive indices and temperature coefficients of THN, IBB, and $n \mathrm{C}_{12}$ at temperatures of T= (293.15 to 318.15) $\mathrm{K}$ wavelength of $513.9 \mathrm{~nm}$ at atmospheric pressure $\mathrm{P}=0.1 \mathrm{MPa}{ }^{\mathrm{a}}$.

\begin{tabular}{|c|c|c|c|c|c|c|c|c|c|}
\hline \multicolumn{3}{|c|}{ Mole Fraction } & \multicolumn{6}{|c|}{ Wavelength $\lambda=513.9 \mathrm{~nm}$} & \multirow{2}{*}{$(\mathrm{dn} / \mathbf{d T})$} \\
\hline THN & IBB & $n \mathrm{C}_{12}$ & 293.15 & 298.15 & 303.15 & 308.15 & 313.15 & 318.15 & \\
\hline 0 & 0 & 1 & 1.42506 & 1.42291 & 1.42074 & 1.41857 & 1.41638 & 1.41418 & $-4.35 \mathrm{E}-04$ \\
\hline 0 & 1 & 0 & 1.4927 & 1.49023 & 1.48772 & 1.48521 & 1.48268 & 1.48023 & $-5.00 \mathrm{E}-04$ \\
\hline \multirow[t]{2}{*}{1} & 0 & 0 & 1.54872 & 1.54625 & 1.54373 & 1.5413 & 1.53886 & 1.53647 & $-4.91 \mathrm{E}-04$ \\
\hline & \multicolumn{8}{|c|}{ Binary mixtures of $\left(\mathrm{n}_{\mathrm{IBB}}\right) n \mathrm{C}_{12}$} & \\
\hline 0 & 0.9014 & 0.0986 & 1.48487 & 1.48229 & 1.47981 & 1.47731 & 1.47502 & 1.47245 & $-4.94 \mathrm{E}-04$ \\
\hline 0 & 0.8024 & 0.1976 & 1.47693 & 1.4745 & 1.47203 & 1.46958 & 1.46712 & 1.46465 & $-4.91 \mathrm{E}-04$ \\
\hline 0 & 0.7032 & 0.2968 & 1.46959 & 1.46722 & 1.46477 & 1.46239 & 1.4599 & 1.45744 & $-4.86 \mathrm{E}-04$ \\
\hline 0 & 0.6036 & 0.3964 & 1.46247 & 1.46005 & 1.45769 & 1.45531 & 1.45293 & 1.45049 & $-4.78 \mathrm{E}-04$ \\
\hline 0 & 0.5038 & 0.4962 & 1.45559 & 1.45312 & 1.45077 & 1.44841 & 1.44611 & 1.44389 & $-4.68 \mathrm{E}-04$ \\
\hline 0 & 0.4036 & 0.5964 & 1.44889 & 1.44665 & 1.44421 & 1.44199 & 1.43955 & 1.43729 & $-4.66 \mathrm{E}-04$ \\
\hline 0 & 0.3032 & 0.6968 & 1.44273 & 1.44037 & 1.43818 & 1.43562 & 1.43332 & 1.43094 & $-4.72 \mathrm{E}-04$ \\
\hline 0 & 0.2024 & 0.7976 & 1.4365 & 1.43425 & 1.43187 & 1.42966 & 1.42725 & 1.42512 & $-4.58 \mathrm{E}-04$ \\
\hline \multirow[t]{2}{*}{0} & 0.1014 & 0.8986 & 1.4307 & 1.42851 & 1.42624 & 1.42401 & 1.42179 & 1.41953 & $-4.47 \mathrm{E}-04$ \\
\hline & \multicolumn{8}{|c|}{ Binary mixtures of $\left(n_{\mathrm{THN}}\right) n \mathrm{C}_{12}$} & \\
\hline 0.1014 & 0 & 0.8986 & 1.43473 & 1.43249 & 1.43024 & 1.42811 & 1.42576 & 1.42366 & $-4.44 \mathrm{E}-04$ \\
\hline 0.2024 & 0 & 0.7976 & 1.44489 & 1.44267 & 1.44033 & 1.43794 & 1.43568 & 1.43333 & $-4.64 \mathrm{E}-04$ \\
\hline 0.3032 & 0 & 0.6968 & 1.45538 & 1.4533 & 1.45084 & 1.44864 & 1.44614 & 1.44405 & $-4.59 \mathrm{E}-04$ \\
\hline 0.4036 & 0 & 0.5964 & 1.46671 & 1.46438 & 1.46216 & 1.45985 & 1.45759 & 1.45487 & $-4.68 \mathrm{E}-04$ \\
\hline 0.5038 & 0 & 0.4962 & 1.47843 & 1.47612 & 1.47359 & 1.47118 & 1.46885 & 1.46645 & $-4.81 \mathrm{E}-04$ \\
\hline 0.6036 & 0 & 0.3964 & 1.49106 & 1.48874 & 1.48633 & 1.48392 & 1.48167 & 1.47929 & $-4.71 \mathrm{E}-04$ \\
\hline 0.7032 & 0 & 0.2968 & 1.50417 & 1.50164 & 1.49917 & 1.49684 & 1.49443 & 1.49184 & $-4.89 \mathrm{E}-04$ \\
\hline
\end{tabular}




\begin{tabular}{|c|c|c|c|c|c|c|c|c|c|}
\hline 3024 & 0 & 1976 & 1.51804 & & & & 1.50825 & 11 & \\
\hline 0014 & & 0986 & .53275 & 1.53046 & 1.52785 & 1.52559 & .52307 & .52083 & $-480 \mathrm{~F}_{-}-04$ \\
\hline \multicolumn{10}{|c|}{ Binary mixtures of $\left(\mathbf{n}_{\mathrm{THN}}\right)$ IBB } \\
\hline 1014 & 0.8986 & 0 & 1.49809 & 1.49546 & 1.49315 & 1.49045 & .48816 & 1.48564 & $-4.96 \mathrm{E}-04$ \\
\hline 2024 & .7976 & 0 & .50316 & 1.50075 & 1.49828 & 1.49591 & .49321 & .49078 & $-4.97 \mathrm{E}-04$ \\
\hline 3032 & & 0 & & 1.50623 & 1.50376 & & .49885 & .49611 & $-5.02 \mathrm{E}-04$ \\
\hline 4036 & & 0 & & & & & & & \\
\hline 5038 & .4962 & 0 & 51959 & 1.51711 & 1.5146 & 1.51215 & .50976 & .50751 & $-4.85 \mathrm{E}-04$ \\
\hline 0050 & & 0 & & & & & & & \\
\hline 70 & & 0 & & & & & & & \\
\hline 8024 & 0.1976 & 0 & 1.5369 & 1.53447 & & 1.52953 & .52715 & 52466 & $-4.89 \mathrm{E}-04$ \\
\hline & & & & & & & & & \\
\hline \multicolumn{10}{|c|}{ Ternary mixtures of $\mathrm{THN}|\mathrm{IBB}| n \mathrm{C}_{12}$} \\
\hline 0.122 & 0.1202 & & 1.44078 & 1.4385 & 1.43627 & 1.43406 & & & \\
\hline 0.119 & 0.2344 & .6466 & & & & & & & \\
\hline .2376 & 0.117 & 0.6454 & & & & & .44226 & & \\
\hline 161 & 0.3431 & 0.5408 & & & & & & & \\
\hline 3472 & & 5389 & & & & & & & \\
\hline 1134 & 0.4467 & & 1.46015 & 1.45781 & 1.45538 & 1.45274 & & & \\
\hline 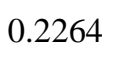 & 0.3344 & 0.4392 & & & & & & & \\
\hline 339 & 0.2226 & 4385 & & & & & 975 & & \\
\hline 4512 & 0.1111 & 4377 & & & & & & & \\
\hline & & & & & & & & & \\
\hline 0.3311 & 0.3262 & 0.3427 & 1.4 & & 1.47139 & & 549 & & \\
\hline 5501 & 0.1084 & 0.3416 & 1.48596 & & & & .47577 & & $-5.00 \mathrm{~F}$ \\
\hline 1002 & & & & & & & & & \\
\hline 0.2161 & 0.5322 & 0.2516 & 1.47915 & 1.47671 & 1.47439 & 1.47174 & 1.46934 & 1.46691 & -4 \\
\hline 3237 & 0.4251 & 0.2512 & 1.48392 & & 1.47918 & & 1.47445 & .4719 & \\
\hline 4309 & & & & & & & & & \\
\hline 0.5377 & 0.2119 & 0.2504 & 1.49386 & 153 & 1.48904 & 1.48671 & 1.48433 & 1.48198 & $6 \mathrm{E}-04$ \\
\hline 6412 & 0.1058 & 25 & 1.49871 & 1.49638 & 1.49395 & 1.49161 & 1.48918 & 1.48691 & $-4.74 \mathrm{E}-0$ \\
\hline 1059 & 0.7298 & 1643 & & & & 459 & 216 & & \\
\hline 0.3166 & 0.5197 & & & & 689 & & 204 & 1.47972 & -4 \\
\hline 0.5259 & 0.3108 & 0.1633 & 1.50208 & 1.49971 & 1.49731 & 1.49479 & 1.49237 & 1.48982 & $-4.91 \mathrm{E}-\mathrm{C}$ \\
\hline 0.734 & 0.1033 & 0.1628 & 1.51233 & 1.51008 & 1.50762 & 1.50512 & 1.50275 & 1.50048 & $-4.79 \mathrm{E}-04$ \\
\hline 0.1036 & 0.8161 & 0.0804 & 1.48972 & 1.48722 & 1.48478 & 1.48232 & 1.47985 & 1.47755 & $-4.88 \mathrm{E}-04$ \\
\hline & 0.7129 & 0.0803 & 1.49499 & 1.49248 & 1.49001 & 1.48741 & 1.48512 & 1.48276 & $-4.90 \mathrm{E}-04$ \\
\hline
\end{tabular}




$\begin{array}{llllllllll}0.3097 & 0.6101 & 0.0801 & 1.50007 & 1.49759 & 1.4951 & 1.4925 & 1.49016 & 1.48762 & -4.98 \mathrm{E}-04 \\ 0.4123 & 0.5077 & 0.08 & 1.50544 & 1.50288 & 1.50024 & 1.49775 & 1.49518 & 1.49258 & -5.14 \mathrm{E}-04 \\ 0.5146 & 0.4055 & 0.0799 & 1.51052 & 1.50801 & 1.50523 & 1.50287 & 1.50045 & 1.49811 & -4.98 \mathrm{E}-04 \\ 0.6166 & 0.3037 & 0.0798 & 1.5161 & 1.51382 & 1.51129 & 1.50864 & 1.50626 & 1.50386 & -4.94 \mathrm{E}-04 \\ 0.7182 & 0.2021 & 0.0796 & 1.52115 & 1.51919 & 1.51696 & 1.51437 & 1.51178 & 1.5096 & -4.72 \mathrm{E}-04 \\ 0.8196 & 0.1009 & 0.0795 & 1.52713 & 1.52479 & 1.52229 & 1.51992 & 1.51752 & 1.51512 & -4.81 \mathrm{E}-04 \\ 0.2318 & 0.2284 & 0.5398 & 1.45767 & 1.45533 & 1.45304 & 1.45073 & 1.44841 & 1.44588 & -4.69 \mathrm{E}-04 \\ 0.6301 & 0.2069 & 0.163 & 1.50675 & 1.50424 & 1.50178 & 1.49933 & 1.49686 & 1.49448 & -4.91 \mathrm{E}-04 \\ 0.2114 & 0.6246 & 0.1641 & 1.48669 & 1.48421 & 1.4818 & 1.47947 & 1.47693 & 1.47444 & -4.88 \mathrm{E}-04 \\ 0.2211 & 0.4356 & 0.3433 & 1.47191 & 1.46932 & 1.46695 & 1.46428 & 1.46153 & 1.45933 & -5.08 \mathrm{E}-04 \\ 0.4408 & 0.2171 & 0.3421 & 1.48102 & 1.47868 & 1.47638 & 1.47384 & 1.47152 & 1.46876 & -4.88 \mathrm{E}-04 \\ 0.4214 & 0.4151 & 0.1635 & 1.49696 & 1.49446 & 1.49223 & 1.48972 & 1.48733 & 1.48494 & -4.80 \mathrm{E}-04\end{array}$

${ }^{a}$ The reported average expanded uncertainty $U=1.197 \times 10^{-3}$ was calculated based on the coverage factor $k=2$, providing a level of confidence of approximately $95 \%$.

Appendix 3.4: Refractive indices and temperature coefficients of $\mathrm{THN}$, IBB, and $n \mathrm{C}_{12}$ at temperatures of $\mathrm{T}=$ (293.15 to 318.15) $\mathrm{K}$ wavelength of $436.1 \mathrm{~nm}$ at atmospheric pressure $\mathrm{P}=0.1 \mathrm{MPa}^{\mathrm{a}}$.

\begin{tabular}{|c|c|c|c|c|c|c|c|c|c|}
\hline \multicolumn{3}{|c|}{ Mole Fraction } & \multicolumn{6}{|c|}{ Wavelength $\lambda=436.1 \quad \mathrm{~nm}$} & \multirow{2}{*}{$(\mathrm{dn} / \mathrm{dT})$} \\
\hline THN & IBB & $n \mathrm{C}_{12}$ & 293.15 & 298.15 & 303.15 & 308.15 & 313.15 & 318.15 & \\
\hline 0 & 0 & 1 & 1.43105 & 1.42886 & 1.42666 & 1.42445 & 1.42223 & 1.42001 & $-4.42 \mathrm{E}-04$ \\
\hline 0 & 1 & 0 & 1.50429 & 1.50172 & 1.49916 & 1.49659 & 1.494 & 1.49143 & $-5.14 \mathrm{E}-04$ \\
\hline 1 & 0 & 0 & 1.56232 & 1.5598 & 1.55719 & 1.55471 & 1.55219 & 1.54974 & $-5.04 \mathrm{E}-04$ \\
\hline \multicolumn{10}{|c|}{ Binary mixtures of $\left(\mathrm{n}_{\mathrm{IBB}}\right) n \mathrm{C}_{12}$} \\
\hline 0 & 0.9014 & 0.0986 & 1.49556 & 1.49284 & 1.49024 & 1.48775 & 1.48511 & 1.48247 & $-5.21 \mathrm{E}-04$ \\
\hline 0 & 0.8024 & 0.1976 & 1.48711 & 1.48462 & 1.48209 & 1.47955 & 1.47706 & 1.47452 & $-5.04 \mathrm{E}-04$ \\
\hline 0 & 0.7032 & 0.2968 & 1.47917 & 1.47676 & 1.47424 & 1.47181 & 1.46924 & 1.46673 & $-4.98 \mathrm{E}-04$ \\
\hline 0 & 0.6036 & 0.3964 & 1.47144 & 1.46903 & 1.4666 & 1.46416 & 1.46172 & 1.45921 & $-4.89 \mathrm{E}-04$ \\
\hline 0 & 0.5038 & 0.4962 & 1.46405 & 1.46153 & 1.45929 & 1.45671 & 1.45448 & 1.4518 & $-4.86 \mathrm{E}-04$ \\
\hline 0 & 0.4036 & 0.5964 & 1.45682 & 1.45454 & 1.45204 & 1.44977 & 1.4473 & 1.44498 & $-4.75 \mathrm{E}-04$ \\
\hline 0 & 0.3032 & 0.6968 & 1.45015 & 1.44775 & 1.44552 & 1.44293 & 1.44059 & 1.43791 & $-4.87 \mathrm{E}-04$ \\
\hline 0 & 0.2024 & 0.7976 & 1.44344 & 1.44124 & 1.43872 & 1.43663 & 1.43404 & 1.43204 & $-4.61 \mathrm{E}-04$ \\
\hline 0 & 0.1014 & 0.8986 & 1.43714 & 1.43491 & 1.43262 & 1.43035 & 1.42808 & 1.42579 & $-4.54 \mathrm{E}-04$ \\
\hline \multicolumn{10}{|c|}{ Binary mixtures of $\left(\mathrm{n}_{\mathrm{THN}}\right) n \mathrm{C}_{12}$} \\
\hline 0.1014 & 0 & 0.8986 & 1.44127 & 1.439 & 1.43671 & 1.43455 & 1.43218 & 1.43001 & $-4.51 \mathrm{E}-04$ \\
\hline 0.2024 & 0 & 0.7976 & 1.45203 & 1.44978 & 1.44738 & 1.44495 & 1.44283 & 1.44026 & $-4.69 \mathrm{E}-04$ \\
\hline 0.3032 & 0 & 0.6968 & 1.46314 & 1.46073 & 1.45818 & 1.45589 & 1.45339 & 1.45084 & $-4.90 \mathrm{E}-04$ \\
\hline 0.4036 & 0 & 0.5964 & 1.47515 & 1.47277 & 1.4705 & 1.46783 & 1.46524 & 1.46269 & $-5.00 \mathrm{E}-04$ \\
\hline
\end{tabular}




\begin{tabular}{|c|c|c|c|c|c|c|c|c|c|}
\hline 5038 & 0 & 4962 & 48812 & 1.48561 & 1.48295 & .4805 & 47822 & 47578 & ש \\
\hline 5036 & & 3964 & 50126 & 1.49875 & 1.49619 & .49371 & 49111 & .48857 & $-5.08 \mathrm{E}-04$ \\
\hline & 0 & & 51481 & & & 1.50749 & & 50241 & E-04 \\
\hline & 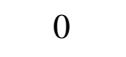 & & & & & & & & E-04 \\
\hline & & & 54558 & & & & 1.5354 & & $-5.00 \mathrm{E}-04$ \\
\hline \multicolumn{10}{|c|}{ Binary mixtures of $\left(n_{\mathrm{THN}}\right)$ IBB } \\
\hline 1014 & 8986 & 0 & 1.50977 & 1.50717 & 1.50468 & 1.50202 & & & $-5.17 \mathrm{E}-04$ \\
\hline 2024 & .7976 & 0 & & 1.51248 & & 1.50759 & 1.5048 & 1.50247 & $-5.03 \mathrm{E}-04$ \\
\hline & 6968 & 0 & 52078 & & & & 1.51044 & 1.50783 & $0 \mathrm{E}-04$ \\
\hline 1036 & 5964 & 0 & & & & & & & \\
\hline & 4962 & 0 & & & & & & & 7E-04 \\
\hline 6036 & 3964 & 0 & 53797 & 1.53551 & 1.533058 & 1.53042 & 1.52806 & 1.52533 & $4 \mathrm{E}-04$ \\
\hline 7032 & .2968 & 0 & & 1.541441 & & 1.53639 & & & $6 \mathrm{E}-04$ \\
\hline & 1976 & 0 & & & & & & & E-04 \\
\hline & & & 1.55611 & & & & & & \\
\hline \multicolumn{10}{|c|}{ Ternary mixtures of THN|IBB $\mid n \mathrm{C}_{12}$} \\
\hline 0.122 & 0.1202 & 0.7578 & & & & 1.44096 & & & $-4.57 \mathrm{E}-04$ \\
\hline 119 & .2344 & 0.6466 & 1.45445 & & 1.44979 & & 1.44517 & & 7E-04 \\
\hline 2376 & 0.117 & 0.6454 & 1.45897 & 1.45656 & 1.45437 & 1.4519 & 1.44974 & 1.44723 & $-4.66 \mathrm{E}-04$ \\
\hline 1161 & 0.3431 & 0.5408 & 1.46156 & & 1.70 & 1.45455 & 1.45209 & & E-04 \\
\hline 372 & .114 & 0.5389 & 1.47057 & & & & & & $5 \mathrm{E}-04$ \\
\hline 1134 & 0.4467 & & 1.46874 & 1.46624 & 1.46387 & 1.46134 & 1.45907 & .45663 & $3 \mathrm{E}-04$ \\
\hline 0.2264 & 0.3344 & 0.4392 & 1.47324 & & & & & & $9 \mathrm{E}-04$ \\
\hline 339 & 0.2226 & 0.4385 & 1.47803 & 1.47569 & 4732 & 1.47086 & & 605 & E-04 \\
\hline 4512 & 0.1111 & .4377 & & & & & & & $2 \mathrm{E}-04$ \\
\hline 1107 & .5454 & 0.3438 & 1.47634 & & & & & & $-4.96 \mathrm{E}-04$ \\
\hline 3311 & 0.3262 & 0.3427 & 1.48573 & 1.483 & 1.48052 & 1.47804 & 757 & .47304 & $5 \mathrm{E}-04$ \\
\hline 5501 & & & & & & & & & $-5.22 \mathrm{E}-04$ \\
\hline 1082 & 0.6397 & .252 & & & & & & & $-5.03 \mathrm{E}-04$ \\
\hline 2161 & 0.5322 & 0.2516 & 1.48912 & 1.48681 & 1.48418 & 1.48161 & 1.4793 & 1.47669 & $-4.99 \mathrm{E}-04$ \\
\hline 3237 & 0.4251 & 0.2512 & 1.49403 & 1.49161 & 1.48914 & 1.4866 & 1.48428 & 1.48169 & $-4.93 \mathrm{E}-04$ \\
\hline 0.4309 & 0.3183 & & & & & & & & $-5.08 \mathrm{E}-04$ \\
\hline 5377 & 0.2119 & 0.2504 & 1.50431 & 1.5019 & 1.49936 & 1.49696 & 1.49452 & 1.49202 & $-4.91 \mathrm{E}-04$ \\
\hline 6442 & 0.1058 & & 1.50925 & 1.50675 & 1.5043 & 1.50162 & 1.49933 & 1.4965 & $-5.07 \mathrm{E}-04$ \\
\hline 0.1059 & 0.7298 & 0.1643 & 1.49222 & 8972 & 872 & 1.48475 & 8227 & 1.47966 & $-5.01 \mathrm{E}-04$ \\
\hline 0.3166 & 0.5197 & & & & & & & & $-5.00 \mathrm{E}-04$ \\
\hline .5259 & 0.3108 & 0.1633 & 1.5132 & 1.51074 & 1.50827 & 1.50554 & 1.50312 & 1.50048 & $-5.10 \mathrm{E}-04$ \\
\hline
\end{tabular}




\begin{tabular}{cccccccccc}
0.734 & 0.1033 & 0.1628 & 1.52404 & 1.52147 & 1.51899 & 1.51651 & 1.514 & 1.51163 & $-4.97 \mathrm{E}-04$ \\
0.1036 & 0.8161 & 0.0804 & 1.50067 & 1.49817 & 1.49552 & 1.49291 & 1.4904 & 1.4878 & $-5.16 \mathrm{E}-04$ \\
0.2068 & 0.7129 & 0.0803 & 1.50625 & 1.50361 & 1.50108 & 1.49855 & 1.49594 & 1.49325 & $-5.17 \mathrm{E}-04$ \\
0.3097 & 0.6101 & 0.0801 & 1.51146 & 1.5089 & 1.50636 & 1.50372 & 1.50125 & 1.49867 & $-5.12 \mathrm{E}-04$ \\
0.4123 & 0.5077 & 0.08 & 1.51642 & 1.51391 & 1.51119 & 1.50892 & 1.50614 & 1.50352 & $-5.15 \mathrm{E}-04$ \\
0.5146 & 0.4055 & 0.0799 & 1.52229 & 1.51975 & 1.51687 & 1.51442 & 1.51165 & 1.50943 & $-5.20 \mathrm{E}-04$ \\
0.6166 & 0.3037 & 0.0798 & 1.52809 & 1.52581 & 1.52313 & 1.5204 & 1.51824 & 1.51549 & $-5.05 \mathrm{E}-04$ \\
0.7182 & 0.2021 & 0.0796 & 1.53405 & 1.53131 & 1.52838 & 1.52581 & 1.52342 & 1.52102 & $-5.22 \mathrm{E}-04$ \\
0.8196 & 0.1009 & 0.0795 & 1.53953 & 1.53713 & 1.53454 & 1.5321 & 1.52964 & 1.52718 & $-4.95 \mathrm{E}-04$ \\
0.2318 & 0.2284 & 0.5398 & 1.46587 & 1.46348 & 1.46114 & 1.45878 & 1.45642 & 1.45408 & $-4.71 \mathrm{E}-04$ \\
0.6301 & 0.2069 & 0.163 & 1.51816 & 1.51549 & 1.51292 & 1.51035 & 1.50787 & 1.50526 & $-5.14 \mathrm{E}-04$ \\
0.2114 & 0.6246 & 0.1641 & 1.49726 & 1.49471 & 1.49222 & 1.48985 & 1.48719 & 1.48448 & $-5.08 \mathrm{E}-04$ \\
0.2211 & 0.4356 & 0.3433 & 1.48096 & 1.47843 & 1.47563 & 1.47341 & 1.47083 & 1.46849 & $-4.99 \mathrm{E}-04$ \\
0.4408 & 0.2171 & 0.3421 & 1.49067 & 1.48814 & 1.48562 & 1.48304 & 1.48053 & 1.47792 & $-5.09 \mathrm{E}-04$ \\
0.4214 & 0.4151 & 0.1635 & 1.5078 & 1.50532 & 1.5029 & 1.50036 & 1.498 & 1.49554 & $-4.90 \mathrm{E}-04$ \\
\hline
\end{tabular}

a The reported average expanded uncertainty $U=1.197 \times 10^{-3}$ was calculated based on the coverage factor $k=2$, providing a level of confidence of approximately $95 \%$.

The average standard deviation comparisons of the temperature coefficient of the refractive index with available literature [15] were presented in table 3.7 .

Appendix 4.1 Average experimental values of refractive indices of $\left(n_{T H N}\right)_{C_{12}} \mid I B B$ and $\left(n_{C_{12}}\right)_{I B B} \mid T H N$ for the (0.501-0.110-0.389) ${ }^{a}$ system at different wavelengths and temperatures ${ }^{b}$ at atmospheric pressure $P=0.1$ $\mathrm{MPa}^{*}$.

\begin{tabular}{|c|c|c|c|c|c|c|c|c|c|c|c|c|}
\hline \multirow{2}{*}{ THN } & \multicolumn{6}{|c|}{$\left(\mathrm{n}_{\mathrm{THN}}\right)_{\mathrm{C} 12} \mid \mathrm{IBB}=0.110$} & \multirow{2}{*}{ IBB } & \multicolumn{5}{|c|}{$\left(\mathrm{n}_{\mathrm{IBB}}\right)_{\mathrm{THN}} \mid \mathrm{C}_{12}=0.389$} \\
\hline & $293 \mathrm{~K}$ & $298 \mathrm{~K}$ & $303 \mathrm{~K}$ & $308 \mathrm{~K}$ & $313 \mathrm{~K}$ & $318 \mathrm{~K}$ & & $298 \mathrm{~K}$ & $303 \mathrm{~K}$ & $308 \mathrm{~K}$ & $313 \mathrm{~K}$ & $318 \mathrm{~K}$ \\
\hline 0.457 & 1.46673 & 1.46435 & 1.46189 & 1.45958 & 1.45718 & 1.45485 & 0.154 & 1.46608 & 1.46382 & 1.46148 & 1.45923 & 1.45689 \\
\hline 0.468 & 1.46786 & 1.46546 & 1.46309 & 1.46075 & 1.45845 & 1.45608 & 0.143 & 1.46679 & 1.46445 & 1.46216 & 1.45985 & 1.45756 \\
\hline 0.49 & 1.46998 & 1.46771 & 1.46535 & 1.46304 & 1.46051 & 1.45825 & 0.121 & 1.46813 & 1.46582 & 1.46353 & 1.46119 & 1.45890 \\
\hline 0.501 & 1.47122 & 1.46882 & 1.46649 & 1.46402 & 1.46166 & 1.45938 & 0.11 & 1.46893 & 1.46661 & 1.46431 & 1.46198 & 1.45962 \\
\hline 0.512 & 1.47240 & 1.47009 & 1.46775 & 1.46534 & 1.46286 & 1.46079 & 0.099 & 1.46966 & 1.46734 & 1.46504 & 1.46273 & 1.46040 \\
\hline 0.523 & 1.47367 & 1.47138 & 1.46907 & 1.46653 & 1.46437 & 1.46210 & 0.088 & 1.47036 & 1.46802 & 1.46570 & 1.46339 & 1.46103 \\
\hline
\end{tabular}




\begin{tabular}{|c|c|c|c|c|c|c|c|c|c|c|c|c|}
\hline 0.556 & .47704 & 1.47459 & 1.47233 & 1.47009 & 1.46768 & 1.46546 & 0.055 & 1.47249 & 1.47018 & 1.46789 & 1.46556 & 1.46323 \\
\hline & \multicolumn{12}{|c|}{$\lambda=632.9 \mathrm{~nm}$} \\
\hline 0.446 & 1.46643 & 1.46411 & 1.46182 & 1.45950 & 1.45720 & 1.45491 & 0.165 & 1.46635 & 1.46438 & 1.46216 & 1.45987 & 1.45759 \\
\hline 0.457 & 1.46749 & 1.46512 & 1.46280 & 1.46052 & 1.45824 & 1.45592 & 0.154 & 1.46710 & 1.46505 & 1.46285 & 1.46053 & 1.45826 \\
\hline 0.468 & 1.46864 & 1.46631 & 1.46399 & 1.46168 & 1.45934 & 1.45692 & 0.143 & 1.46779 & 1.46555 & 1.46330 & 1.46103 & 1.45877 \\
\hline 0.479 & 1.46988 & 1.46755 & 1.46528 & 1.46302 & 1.46074 & 1.45847 & 0.132 & 1.46847 & 1.46618 & 1.46391 & 1.46161 & 1.45934 \\
\hline 0.49 & 1.47106 & 1.46872 & 1.46647 & 1.46421 & 1.46193 & 1.45963 & 0.121 & 1.46923 & 1.46702 & 1.46472 & 1.46245 & 1.46015 \\
\hline 0.501 & 1.47234 & 1.46998 & 1.46771 & 1.46543 & 1.46316 & 1.46088 & 0.11 & 1.46984 & 1.46760 & 1.46533 & 1.46307 & 1.46080 \\
\hline 0.512 & 1.47347 & 1.47114 & 1.46888 & 1.46662 & 1.46436 & 1.46205 & 0.099 & 1.47051 & 1.46825 & 1.46597 & 1.46368 & 1.46134 \\
\hline 0.523 & 1.47451 & 1.47220 & 1.46993 & 1.46766 & 1.46537 & 1.46308 & 0.088 & 1.47111 & 1.46878 & 1.46657 & 1.46429 & 1.46199 \\
\hline 0.534 & 1.47573 & 1.47341 & 1.47114 & 1.46888 & 1.46660 & 1.46431 & 0.077 & 1.47178 & 1.46962 & 1.46726 & 1.46498 & 1.46269 \\
\hline 0.545 & 1.47705 & 1.47477 & 1.47251 & 1.47025 & 1.46799 & 1.46569 & 0.066 & 1.47271 & 1.47025 & 1.46797 & 1.46569 & 1.46340 \\
\hline \multirow[t]{2}{*}{0.556} & & 1.47587 & 1.47360 & 1.47136 & 1.46909 & 1.46680 & 0.055 & & & 1.46896 & & 1.46411 \\
\hline & \multicolumn{12}{|c|}{$\lambda=589.2 \mathrm{~nm}$} \\
\hline 0.446 & 1.46889 & 1.46644 & 1.46399 & 1.46149 & 1.45940 & 1.45690 & 0.165 & 1.46845 & 1.46603 & 1.46383 & 6150 & 1.45920 \\
\hline 0.457 & 1.46981 & 1.46753 & 1.46515 & 1.46283 & 1.46047 & 1.45793 & 0.154 & & & & 1.46230 & 1.45994 \\
\hline 0.468 & 1.47102 & 1.46863 & 1.46629 & 1.46395 & 1.46158 & 1.45918 & 0.143 & 1.46998 & 1.46764 & 1.46537 & 1.46302 & 1.46064 \\
\hline 0.479 & 1.47212 & 1.46970 & 1.46746 & 1.46519 & 1.46275 & 1.46044 & 0.132 & 1.47058 & 1.46824 & 1.46598 & & 1.46130 \\
\hline 0.49 & 1.47334 & 1.47098 & 1.46866 & 1.46626 & 1.46403 & 1.46180 & 0.121 & 1.47131 & 1.46896 & & & 1.46205 \\
\hline 0.501 & 1.47440 & 1.47197 & 1.46957 & 1.46722 & 1.46489 & 1.46261 & 0.11 & 1.47212 & 1.46979 & 1.46746 & 1.46515 & 1.46282 \\
\hline 0.512 & 1.47566 & 1.47330 & 1.47096 & 1.46859 & 1.46623 & 1.46387 & 0.099 & 1.47286 & 1.47054 & 1.46821 & 89 & 355 \\
\hline 0.523 & 1.47704 & 1.47458 & 1.47221 & 1.46991 & 1.46753 & 1.46521 & 0.088 & 1.47358 & 1.47123 & 1.46888 & 1.46657 & 1.46423 \\
\hline 0.534 & 1.47809 & 1.47568 & 1.47335 & 1.47103 & 1.46879 & 1.46633 & 0.077 & 1.47427 & 1.47195 & 1.46965 & 1.46728 & 1.46489 \\
\hline 0.545 & & & 1.47458 & & 1.47004 & & & & & & & \\
\hline \multirow[t]{2}{*}{0.556} & & & & & & & 0.055 & & & & & \\
\hline & \multicolumn{12}{|c|}{$\lambda=513.9 \mathrm{~nm}$} \\
\hline 0.446 & 1.47368 & 1.47113 & 1.46880 & 1.46622 & 1.46394 & 1.46155 & 0.165 & 1.47357 & 1.47104 & 1.46882 & & 1.46405 \\
\hline 0.457 & 1.47491 & 1.47252 & 1.46999 & 1.46756 & 1.46519 & 1.46267 & 0.154 & 1.47435 & 1.47189 & 1.46960 & 1.46727 & 1.46492 \\
\hline 0.468 & 1.47615 & 1.47378 & 1.47136 & 1.46901 & & & 0.143 & & 1.47265 & 1.47038 & 1.46799 & 1.46564 \\
\hline 0.479 & 1.47727 & 1.47499 & 1.47244 & 1.47012 & 1.46773 & 1.46538 & 0.132 & 1.47582 & 1.47341 & 1.47109 & 1.46867 & 1.46633 \\
\hline 0.49 & 1.47853 & 1.47605 & 1.47375 & 1.47122 & 1.46888 & 1.46661 & 0.121 & & 1.47412 & 1.47180 & & 1.46708 \\
\hline 0.501 & 1.47963 & & 1.47473 & 1.47231 & 1.46991 & 1.46767 & 0.11 & 1.47734 & 1.47498 & 1.47260 & 1.47033 & 1.46788 \\
\hline 0.512 & 1.48092 & 1.47858 & 1.47621 & 1.47382 & 1.47135 & 1.46909 & 0.099 & 1.47812 & 1.47579 & 1.47338 & 1.47106 & 1.46863 \\
\hline 0.523 & 1.48226 & 1.47986 & 1.47748 & 1.47508 & 1.47256 & 1.47037 & 0.088 & 1.47887 & 1.47648 & 1.47412 & 1.47175 & 1.46932 \\
\hline 0.534 & 1.48342 & 1.48101 & 1.47867 & 1.47632 & 1.47393 & 1.47156 & 0.077 & 1.47957 & 1.47723 & 1.47482 & 1.47246 & 1.47007 \\
\hline 0.545 & 1.48471 & 1.48234 & 1.47994 & 1.47762 & 1.47523 & 1.47289 & 0.066 & 1.48036 & 1.47802 & 1.47563 & 1.47324 & 1.47083 \\
\hline \multirow[t]{2}{*}{0.556} & 1.48581 & 1.48351 & 1.48120 & 1.47886 & 1.47647 & 1.47412 & 0.055 & & & & & \\
\hline & \multicolumn{12}{|c|}{$\lambda=436.1 \mathrm{~nm}$} \\
\hline 0.446 & 1.48278 & 1.48033 & 1.47773 & 1.47537 & 1.47292 & 1.47047 & 0.165 & 1.48248 & 1.48008 & & & 1.47284 \\
\hline 0.457 & 1.48406 & 1.48156 & 1.47905 & 1.47667 & 1.47417 & 1.47177 & 0.154 & 1.48328 & 1.48086 & 1.47836 & 1.47604 & 1.47366 \\
\hline 0.468 & 1.48534 & 1.48289 & 1.48045 & 1.47807 & 1.47561 & 1.47316 & 0.143 & 1.48418 & 1.48174 & 1.47928 & 1.47689 & 1.47444 \\
\hline 0.479 & 1.48654 & 1.48408 & 1.48163 & 1.47927 & 1.47678 & 1.47440 & 0.132 & 1.48499 & 1.48261 & 1.48018 & 1.47771 & 1.47528 \\
\hline
\end{tabular}




\begin{tabular}{ccccccccccccc}
0.49 & 1.48781 & 1.48537 & 1.48292 & 1.48052 & 1.47807 & 1.47564 & 0.121 & 1.48574 & 1.48339 & 1.48094 & 1.47856 & 1.47612 \\
0.501 & 1.48911 & 1.48663 & 1.48419 & 1.48170 & 1.47925 & 1.47680 & 0.11 & 1.48666 & 1.48428 & 1.48181 & 1.47945 & 1.47695 \\
0.512 & 1.49043 & 1.48801 & 1.48559 & 1.48319 & 1.48074 & 1.47838 & 0.099 & 1.48750 & 1.48516 & 1.48268 & 1.48028 & 1.47784 \\
0.523 & 1.49183 & 1.48938 & 1.48695 & 1.48454 & 1.48211 & 1.47969 & 0.088 & 1.48834 & 1.48594 & 1.48349 & 1.48105 & 1.47859 \\
0.534 & 1.49308 & 1.49063 & 1.48821 & 1.48582 & 1.48337 & 1.48095 & 0.077 & 1.48912 & 1.48671 & 1.48430 & 1.48186 & 1.47938 \\
0.545 & 1.49444 & 1.49193 & 1.48955 & 1.48714 & 1.48474 & 1.48231 & 0.066 & 1.48993 & 1.48754 & 1.48513 & 1.48269 & 1.48023 \\
0.556 & 1.49566 & 1.49322 & 1.49076 & 1.48844 & 1.48596 & 1.48359 & 0.055 & 1.49083 & 1.48842 & 1.48601 & 1.48357 & 1.48110 \\
\hline
\end{tabular}

"The reported average expanded uncertainty $U=1.197 \times 10^{-3}$ was calculated based on the coverage factor $k=2$, providing a level of confidence of approximately $95 \%$. ${ }^{a}$ The desired mixtures were prepared using pre-prescribed molar fraction with a sampling rate of $10 \%$ for each component at an accuracy of $\pm 1 \times 10^{-3} \mathrm{~g} / 5 \mathrm{~g}$. ${ }^{\mathrm{b}}$ The temperatures are in K controlled by a Peltier thermostat with temperature sensors at an accuracy of $\pm 0.03 \mathrm{~K}$.

Appendix 4.2: Average experimental values of refractive indices of $\left(n_{T H N}\right)_{n C_{12}} \mid I B B$ and $\left(n_{C_{12}}\right)_{I B B} \mid T H N$ for the (0.40-0.20-0.40) ${ }^{\mathrm{a}}$ system at different wavelengths and temperatures ${ }^{\mathrm{b}}$ at atmospheric pressure $\mathrm{P}=0.1$ $\mathrm{MPa}^{*}$.

$\lambda=657.2 \mathrm{~nm}$

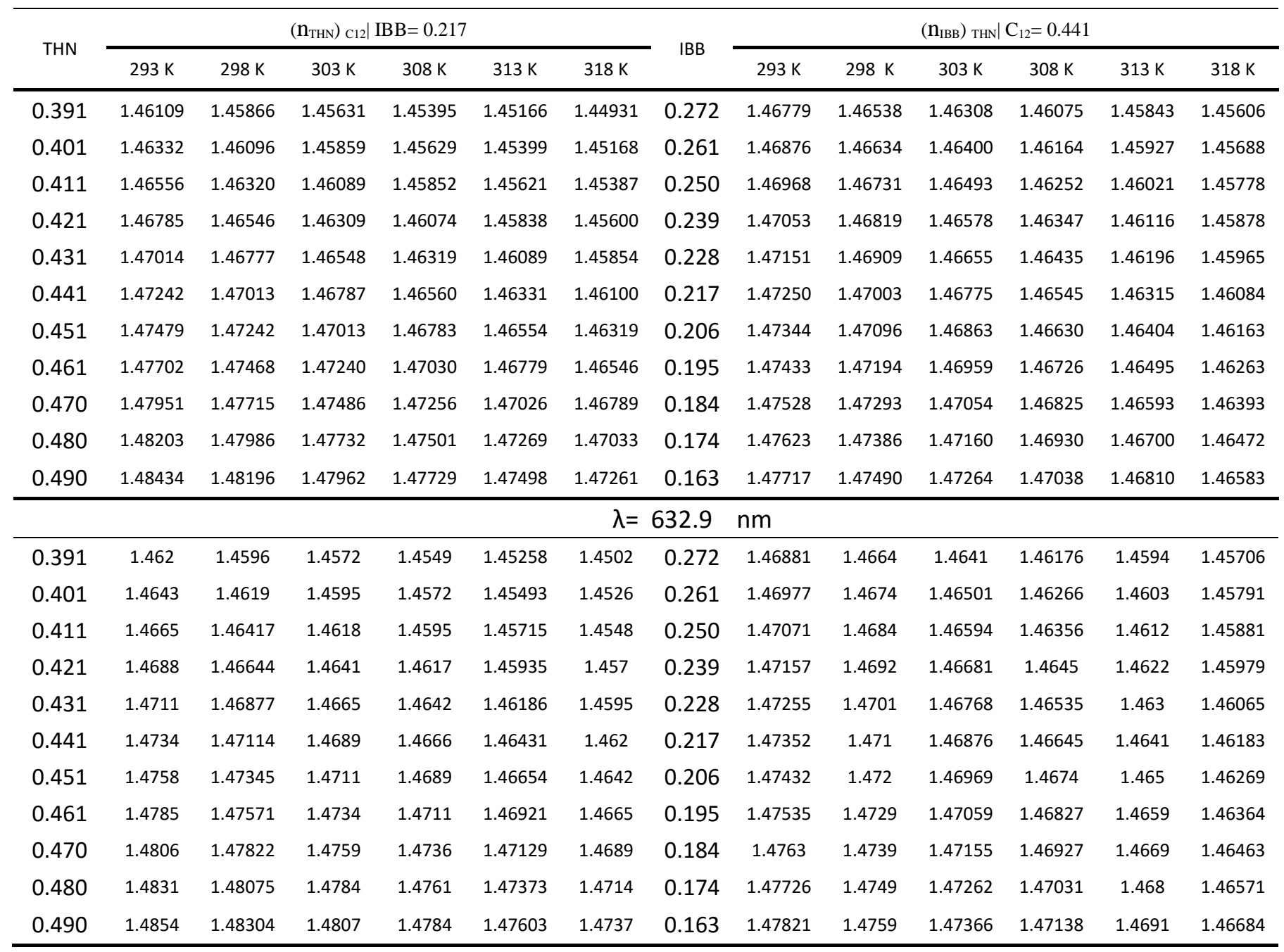




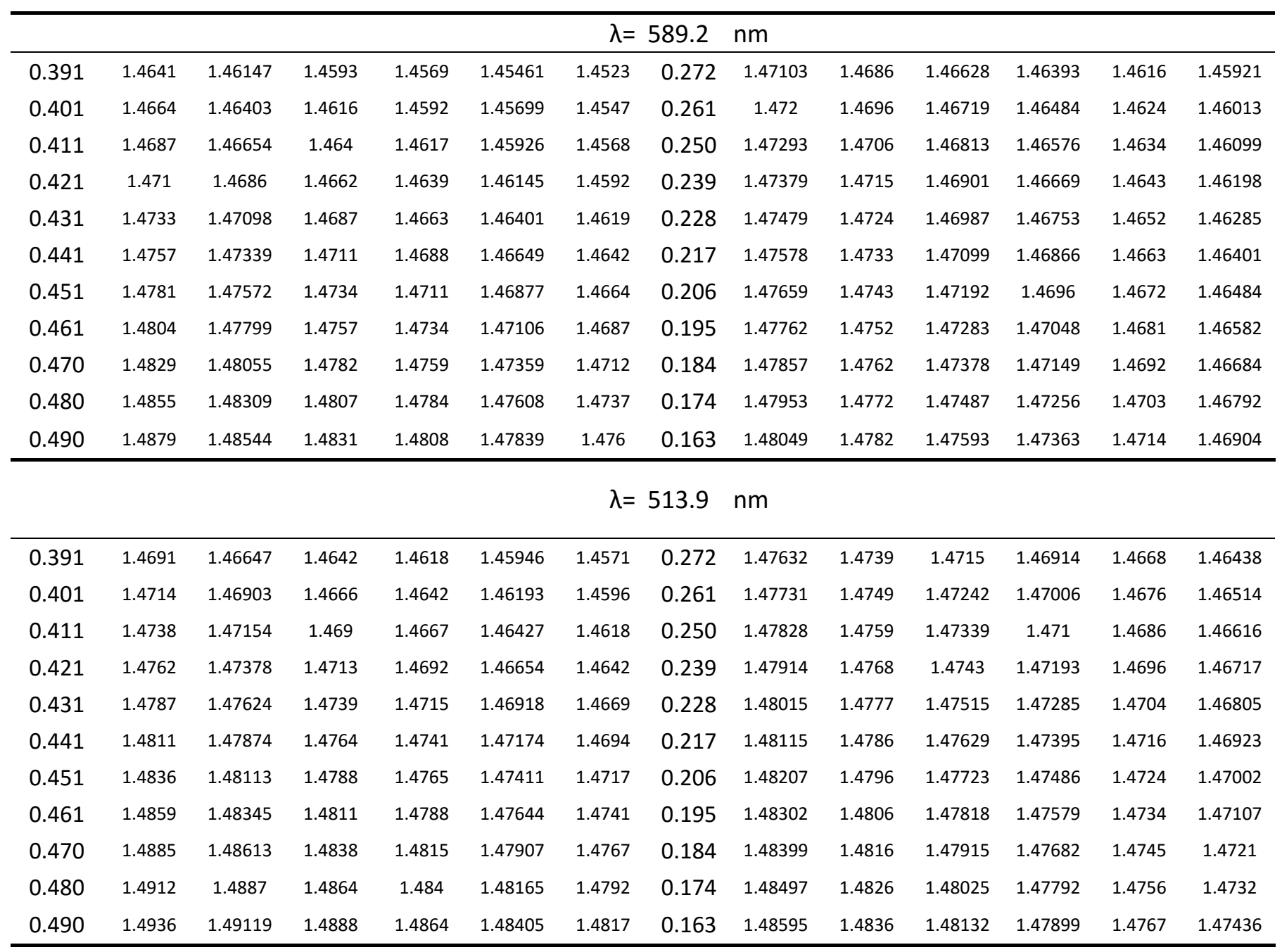

$\lambda=436.1 \mathrm{~nm}$

\begin{tabular}{llllllllllllll}
\hline 0.391 & 1.4779 & 1.47543 & 1.4729 & 1.4705 & 1.4681 & 1.4657 & 0.272 & 1.48580 & 1.48328 & 1.48085 & 1.47842 & 1.47601 & 1.47354 \\
0.401 & 1.4805 & 1.47799 & 1.4755 & 1.4731 & 1.47072 & 1.4683 & 0.261 & 1.48681 & 1.48429 & 1.48180 & 1.47937 & 1.47687 & 1.47442 \\
0.411 & 1.483 & 1.4805 & 1.4781 & 1.4756 & 1.4732 & 1.4708 & 0.250 & 1.48780 & 1.48535 & 1.48280 & 1.48035 & 1.47784 & 1.47539 \\
0.421 & 1.4856 & 1.48305 & 1.4805 & 1.4781 & 1.47562 & 1.4732 & 0.239 & 1.48871 & 1.48626 & 1.48373 & 1.48131 & 1.47885 & 1.47642 \\
0.431 & 1.4881 & 1.48566 & 1.4832 & 1.4809 & 1.4784 & 1.476 & 0.228 & 1.48975 & 1.48721 & 1.48461 & 1.48227 & 1.47977 & 1.47736 \\
0.441 & 1.4907 & 1.48829 & 1.4859 & 1.4835 & 1.48113 & 1.4787 & 0.217 & 1.49078 & 1.48821 & 1.48580 & 1.48340 & 1.48098 & 1.47855 \\
0.451 & 1.4934 & 1.49084 & 1.4885 & 1.4861 & 1.48364 & 1.4812 & 0.206 & 1.49172 & 1.48928 & 1.48678 & 1.48438 & 1.48189 & 1.47935 \\
0.461 & 1.4958 & 1.49325 & 1.4909 & 1.4885 & 1.48607 & 1.4837 & 0.195 & 1.49272 & 1.49020 & 1.48773 & 1.48530 & 1.48288 & 1.48044 \\
0.470 & 1.4987 & 1.49617 & 1.4938 & 1.4914 & 1.48892 & 1.4865 & 0.184 & 1.49373 & 1.49126 & 1.48874 & 1.48637 & 1.48397 & 1.48152 \\
0.480 & 1.5015 & 1.49896 & 1.4965 & 1.4941 & 1.49166 & 1.4892 & 0.174 & 1.49474 & 1.49229 & 1.48989 & 1.48747 & 1.48510 & 1.48267 \\
0.490 & 1.5041 & 1.50154 & 1.4991 & 1.4967 & 1.49422 & 1.4918 & 0.163 & 1.49575 & 1.49337 & 1.49100 & 1.48861 & 1.48625 & 1.48385 \\
\hline
\end{tabular}

" The reported average expanded uncertainty $U=1.197 \times 10^{-3}$ was calculated based on the coverage factor $k=2$, providing a level of confidence of approximately $95 \%$. ${ }^{\text {a }}$ The desired mixtures were prepared using pre-prescribed molar fraction with a sampling rate of $10 \%$ for each component at an accuracy of $\pm 1 \times 10^{-3} \mathrm{~g} / 5 \mathrm{~g}$. ${ }^{\mathrm{b}}$ The temperatures are in K controlled by a Peltier thermostat with temperature sensors at an accuracy of $\pm 0.03 \mathrm{~K}$. 
Appendix 4.3: Average experimental values of refractive indices of $\left(n_{T H N}\right)_{12} \mid I B B$ and $\left(n_{C_{12}}\right)_{I B B} \mid T H N$ for the (0.45-0.10-0.45) and (0.40-0.20-0.40) ${ }^{a}$ system at different wavelengths and temperatures ${ }^{b}$ at atmospheric pressure $\mathbf{P}=0.1 \mathrm{MPa}^{*}$.

\begin{tabular}{|c|c|c|c|c|c|c|c|c|c|c|c|c|c|}
\hline \multirow{3}{*}{ IBB } & \multicolumn{13}{|c|}{$\lambda=657.2 \mathrm{~nm}$} \\
\hline & \multicolumn{6}{|c|}{$\left(\mathrm{n}_{\mathrm{IBB}}\right)_{\mathrm{C} 12} \mid \mathrm{THN}=0.501$} & \multirow{2}{*}{ IBB } & \multicolumn{6}{|c|}{$\left(\mathrm{n}_{\mathrm{IBB}}\right)_{\mathrm{Cl} 2} \mid \mathrm{THN}=0.441$} \\
\hline & $293 \mathrm{~K}$ & $298 \mathrm{~K}$ & $303 \mathrm{~K}$ & $308 \mathrm{~K}$ & $313 \mathrm{~K}$ & $318 \mathrm{~K}$ & & $293 \mathrm{~K}$ & $298 \mathrm{~K}$ & $303 \mathrm{~K}$ & $308 \mathrm{~K}$ & $313 \mathrm{~K}$ & $318 \mathrm{~K}$ \\
\hline 0.163 & 1.46756 & 1.46527 & 1.46297 & 1.46065 & 1.45849 & 1.45628 & 0.268 & 1.47976 & 1.47737 & 1.47495 & 1.47252 & 1.47020 & 1.46783 \\
\hline 0.152 & 1.46831 & 1.46597 & 1.46369 & 1.46148 & 1.45915 & 1.45686 & 0.258 & 1.47822 & 1.47590 & 1.47358 & 1.47118 & 1.46887 & 1.46650 \\
\hline 0.142 & 1.46900 & 1.46668 & 1.46444 & 1.46215 & 1.45994 & 1.45762 & 0.248 & 1.47690 & 1.47443 & 1.47218 & 1.46978 & 1.46744 & 1.46509 \\
\hline 0.131 & 1.46970 & 1.46739 & 1.46506 & 1.46283 & 1.46058 & 1.45836 & 0.238 & 1.47543 & 1.47298 & 1.47065 & 1.46835 & 1.46592 & 1.46365 \\
\hline 0.120 & 1.47036 & 1.46810 & 1.46583 & 1.46355 & 1.46126 & 1.45905 & 0.227 & 1.47395 & 1.47151 & 1.46921 & 1.46682 & 1.46464 & 1.46219 \\
\hline 0.110 & 1.47101 & 1.46876 & 1.46643 & 1.46418 & 1.46185 & 1.45959 & 0.217 & 1.47250 & 1.47017 & 1.46784 & 1.46555 & 1.46321 & 1.46090 \\
\hline 0.099 & 1.47186 & 1.46955 & 1.46720 & 1.46495 & 1.46267 & 1.46034 & 0.207 & 1.47111 & 1.46879 & 1.46647 & 1.46420 & 1.46201 & 1.45959 \\
\hline 0.088 & 1.47276 & 1.47047 & 1.46815 & 1.46578 & 1.46346 & 1.46114 & 0.196 & 1.46974 & 1.46742 & 1.46513 & 1.46285 & 1.46059 & 1.45822 \\
\hline 0.077 & 1.47353 & 1.47125 & 1.46888 & 1.46659 & 1.46430 & 1.46208 & 0.186 & 1.46834 & 1.46599 & 1.46374 & 1.46142 & 1.45922 & 1.45694 \\
\hline 0.066 & 1.47424 & 1.47191 & 1.46953 & 1.46724 & 1.46498 & 1.46273 & 0.175 & 1.46701 & 1.46470 & 1.46255 & 1.46008 & 1.45795 & 1.45556 \\
\hline \multirow[t]{2}{*}{0.056} & 1.47504 & 1.47261 & 1.47034 & 1.46803 & 1.46578 & 1.46345 & 0.165 & 1.46552 & 1.46335 & 1.46110 & 1.45882 & 1.45641 & 1.45434 \\
\hline & \multicolumn{7}{|c|}{$\lambda=632.9$} & \multicolumn{6}{|l|}{$\mathrm{nm}$} \\
\hline 0.163 & 1.46820 & 1.46616 & 1.46397 & 1.46167 & 1.45937 & 1.45715 & 0.268 & 1.48083 & 1.47834 & 1.47611 & 1.47368 & 1.47144 & 1.46899 \\
\hline 0.152 & 1.46896 & 1.46686 & 1.46468 & 1.46246 & 1.46020 & 1.45799 & 0.258 & 1.47938 & 1.47691 & 1.47463 & 1.47232 & 1.47000 & 1.46752 \\
\hline 0.142 & 1.46978 & 1.46748 & 1.46531 & 1.46300 & 1.46078 & 1.45862 & 0.248 & 1.47794 & 1.47554 & 1.47322 & 1.47091 & 1.46858 & 1.46622 \\
\hline 0.131 & 1.47055 & 1.46828 & 1.46599 & 1.46378 & 1.46145 & 1.45923 & 0.238 & 1.47646 & 1.47406 & 1.47184 & 1.46945 & 1.46712 & 1.46483 \\
\hline 0.120 & 1.47145 & 1.46905 & 1.46683 & 1.46453 & 1.46222 & 1.45992 & 0.227 & 1.47497 & 1.47254 & 1.47033 & 1.46799 & 1.46572 & 1.46328 \\
\hline 0.110 & 1.47204 & 1.46986 & 1.46755 & 1.46525 & 1.46295 & 1.46056 & 0.217 & 1.47352 & 1.47116 & 1.46885 & 1.46655 & 1.46430 & 1.46199 \\
\hline 0.099 & 1.47276 & 1.47055 & 1.46820 & 1.46592 & 1.46365 & 1.46132 & 0.207 & 1.47211 & 1.46977 & 1.46748 & 1.46508 & 1.46276 & 1.46047 \\
\hline 0.088 & 1.47375 & 1.47147 & 1.46915 & 1.46678 & 1.46446 & 1.46213 & 0.196 & 1.47065 & 1.46822 & 1.46611 & 1.46372 & 1.46144 & 1.45902 \\
\hline 0.077 & 1.47444 & 1.47225 & 1.46987 & 1.46762 & 1.46528 & 1.46305 & 0.186 & 1.46933 & 1.46675 & 1.46470 & 1.46232 & 1.45998 & 1.45772 \\
\hline 0.066 & 1.47505 & 1.47292 & 1.47069 & 1.46835 & 1.46604 & 1.46373 & 0.175 & 1.46779 & 1.46526 & 1.46309 & 1.46072 & 1.45874 & 1.45632 \\
\hline \multirow[t]{2}{*}{0.056} & 1.47580 & 1.47353 & 1.47138 & 1.46909 & 1.46674 & 1.46459 & 0.165 & 1.46597 & 1.46385 & 1.46154 & 1.45942 & 1.45713 & 1.45483 \\
\hline & \multicolumn{13}{|c|}{$\lambda=589.2$} \\
\hline 0.163 & 1.47057 & 1.46822 & 1.46592 & 1.46375 & 1.46145 & 1.45922 & 0.268 & 1.48324 & 1.48072 & 1.47848 & 1.47604 & 1.47367 & 1.47133 \\
\hline 0.152 & 1.47138 & 1.46904 & 1.46671 & 1.46440 & 1.46215 & 1.45984 & 0.258 & 1.48165 & 1.47940 & 1.47707 & 1.47464 & 1.47230 & 1.46993 \\
\hline 0.142 & 1.47215 & 1.46985 & 1.46749 & 1.46515 & 1.46288 & 1.46055 & 0.248 & 1.48026 & 1.47796 & 1.47563 & 1.47320 & 1.47086 & 1.46848 \\
\hline 0.131 & 1.47296 & 1.47058 & 1.46826 & 1.46591 & 1.46360 & 1.46126 & 0.238 & 1.47867 & 1.47644 & 1.47405 & 1.47165 & 1.46935 & 1.46716 \\
\hline 0.120 & 1.47372 & 1.47135 & 1.46903 & 1.46673 & 1.46435 & 1.46206 & 0.227 & 1.47714 & 1.47489 & 1.47258 & 1.47018 & 1.46795 & 1.46550 \\
\hline 0.110 & 1.47432 & 1.47200 & 1.46984 & 1.46740 & 1.46504 & 1.46269 & 0.217 & 1.47578 & 1.47350 & 1.47119 & 1.46875 & 1.46639 & 1.46407 \\
\hline 0.099 & 1.47508 & 1.47275 & 1.47059 & 1.46815 & 1.46580 & 1.46341 & 0.207 & 1.47433 & 1.47199 & 1.46955 & 1.46736 & 1.46506 & 1.46274 \\
\hline 0.088 & 1.47584 & 1.47357 & 1.47125 & 1.46894 & 1.46662 & 1.46419 & 0.196 & 1.47292 & 1.47052 & 1.46821 & 1.46592 & 1.46374 & 1.46142 \\
\hline 0.077 & 1.47679 & 1.47449 & 1.47210 & 1.46985 & 1.46746 & 1.46511 & 0.186 & 1.47164 & 1.46942 & 1.46715 & 1.46475 & 1.46249 & 1.46025 \\
\hline 0.066 & 1.47763 & 1.47521 & 1.47285 & 1.47050 & 1.46820 & 1.46581 & 0.175 & 1.47024 & 1.46795 & 1.46576 & 1.46349 & 1.46118 & 1.45884 \\
\hline 0.056 & 1.47848 & 1.47602 & 1.47363 & 1.47130 & 1.46900 & 1.46675 & 0.165 & 1.46878 & 1.46655 & 1.46445 & 1.46199 & 1.45979 & 1.45755 \\
\hline
\end{tabular}


$\lambda=513.9 \mathrm{~nm}$

\begin{tabular}{|c|c|c|c|c|c|c|c|c|c|c|c|c|c|}
\hline 0.163 & 1.47585 & 1.47349 & 1.47116 & 1.46895 & 1.46655 & 1.46415 & 0.268 & 1.48894 & 1.48639 & 1.48401 & 1.48164 & 1.47925 & 1.47688 \\
\hline 0.152 & 1.47665 & 1.47428 & 1.47195 & 1.46963 & 1.46720 & 1.46480 & 0.258 & 1.48729 & 1.48491 & 1.48255 & 1.48019 & 1.47783 & 1.47543 \\
\hline 0.142 & 1.47732 & 1.47505 & 1.47265 & 1.47023 & 1.46791 & 1.46551 & 0.248 & 1.48585 & 1.48341 & 1.48104 & 1.47870 & 1.47631 & 1.47392 \\
\hline 0.131 & 1.47815 & 1.47581 & 1.47345 & 1.47108 & 1.46868 & 1.46636 & 0.238 & 1.48416 & 1.48178 & 1.47951 & 1.47719 & 1.47475 & 1.47226 \\
\hline 0.120 & 1.47885 & 1.47654 & 1.47418 & 1.47180 & 1.46944 & 1.46720 & 0.227 & 1.48259 & 1.48020 & 1.47787 & 1.47552 & 1.47316 & 1.47079 \\
\hline 0.110 & 1.47970 & 1.47730 & 1.47489 & 1.47247 & 1.47008 & 1.46782 & 0.217 & 1.48115 & 1.47875 & 1.47639 & 1.47405 & 1.47164 & 1.46929 \\
\hline 0.099 & 1.48055 & 1.47825 & 1.47586 & 1.47338 & 1.47095 & 1.46871 & 0.207 & 1.47963 & 1.47727 & 1.47491 & 1.47258 & 1.47025 & 1.46790 \\
\hline 0.088 & 1.48136 & 1.47897 & 1.47656 & 1.47427 & 1.47185 & 1.46944 & 0.196 & 1.47811 & 1.47577 & 1.47347 & 1.47118 & 1.46888 & 1.46650 \\
\hline 0.077 & 1.48214 & 1.47985 & 1.47736 & 1.47515 & 1.47265 & 1.47028 & 0.186 & 1.47669 & 1.47445 & 1.47216 & 1.46975 & 1.46744 & 1.46496 \\
\hline 0.066 & 1.48309 & 1.48071 & 1.47827 & 1.47584 & 1.47339 & 1.47101 & 0.175 & 1.47526 & 1.47298 & 1.47078 & 1.46838 & 1.46595 & 1.46369 \\
\hline 0.056 & 1.48402 & 1.48154 & 1.47928 & 1.47687 & 1.47445 & 1.47194 & 0.165 & 1.47373 & 1.47132 & 1.46905 & 1.46690 & 1.46462 & 1.46219 \\
\hline & \multicolumn{13}{|c|}{$\lambda=436.1 \mathrm{~nm}$} \\
\hline 0.163 & 1.48503 & 1.48255 & 1.48025 & 1.47776 & 1.47535 & 1.47305 & 0.268 & 1.49922 & 1.49660 & 1.49415 & 1.49172 & 1.48925 & 1.48681 \\
\hline 0.152 & 1.48589 & 1.48348 & 1.48105 & 1.47863 & 1.47625 & 1.47397 & 0.258 & 1.49743 & 1.49497 & 1.49255 & 1.49014 & 1.48769 & 1.48526 \\
\hline 0.142 & 1.48663 & 1.48423 & 1.48179 & 1.47941 & 1.47699 & 1.47475 & 0.248 & 1.49586 & 1.49336 & 1.49093 & 1.48851 & 1.48605 & 1.48361 \\
\hline 0.131 & 1.48732 & 1.48494 & 1.48255 & 1.48017 & 1.47772 & 1.47548 & 0.238 & 1.49417 & 1.49176 & 1.48935 & 1.48695 & 1.48439 & 1.48186 \\
\hline 0.120 & 1.48819 & 1.48584 & 1.48338 & 1.48107 & 1.47862 & 1.47613 & 0.227 & 1.49234 & 1.48989 & 1.48748 & 1.48511 & 1.48267 & 1.48024 \\
\hline 0.110 & 1.48913 & 1.48664 & 1.48423 & 1.48176 & 1.47935 & 1.47688 & 0.217 & 1.49078 & 1.48830 & 1.48591 & 1.48350 & 1.48103 & 1.47861 \\
\hline 0.099 & 1.48991 & 1.48744 & 1.48497 & 1.48256 & 1.48007 & 1.47767 & 0.207 & 1.48915 & 1.48671 & 1.48429 & 1.48192 & 1.47950 & 1.47711 \\
\hline 0.088 & 1.49085 & 1.48843 & 1.48594 & 1.48346 & 1.48092 & 1.47868 & 0.196 & 1.48747 & 1.48504 & 1.48265 & 1.48026 & 1.47789 & 1.47557 \\
\hline 0.077 & 1.49174 & 1.48934 & 1.48687 & 1.48442 & 1.48184 & 1.47955 & 0.186 & 1.48596 & 1.48365 & 1.48112 & 1.47873 & 1.47637 & 1.47407 \\
\hline 0.066 & 1.49255 & 1.49011 & 1.48765 & 1.48517 & 1.48276 & 1.48040 & 0.175 & 1.48439 & 1.48185 & 1.47945 & 1.47702 & 1.47483 & 1.47258 \\
\hline 0.056 & 1.49346 & 1.49095 & 1.48857 & 1.48615 & 1.48373 & 1.48132 & 0.165 & 1.48255 & 1.48006 & 1.47786 & 1.47553 & 1.47300 & 1.47066 \\
\hline
\end{tabular}

* The reported average expanded uncertainty $U=1.197 \times 10^{-3}$ was calculated based on the coverage factor $k=2$, providing a level of confidence of approximately $95 \%$. ${ }^{\text {a }}$ The desired mixtures were prepared using pre-prescribed molar fraction with a sampling rate of $10 \%$ for each component at an accuracy of $\pm 1 \times 10^{-3} \mathrm{~g} / 5 \mathrm{~g}$. ${ }^{\mathrm{b}}$ The temperatures are in K controlled by a Peltier thermostat with temperature sensors at an accuracy of $\pm 0.03 \mathrm{~K}$. 


\section{References}

[1]. Jiménez, RJ., Philipp, M.; Ramos, MA., Krüger, JK. Concentration and temperature dependence of the refractive index of ethanol-water mixtures: Influence of intermolecular interactions. Eur Phys J E. 2009,30,19-26.

[2]. Croccolo, F., Arnaud, M., Bégué, D.; Bataller, H. Concentration dependent refractive index of a binary mixture at high pressure. J Chem Phys. 2011,135,034901-1.

[3]. Narendra, K., Narayanamurthy, P., Srinivasu, C. Refractive indices of binary liquid mixture at different temperatures. Asian J Appl Sci. 2011,4,535-541.

[4]. Gebhardt, M., Köhler, W., Mialdun, A., Yasnou, V., Shevtsova, V. Diffusion, thermal diffusion, and Soret coefficients and optical contrast factors of the binary mixtures of dodecane, isobutylbenzene, and 1,2,3,4-tetrahydronaphthalene. J Chem Phys. 2013,138, 114503-1.

[5]. Sechenyh, V., Legros J., Shevtsova, V. Optical properties of binary and ternary liquid mixtures containing tetralin, isobutylbenzene and dodecane. J. Chem.Thermodyn. 2013,62,64-68.

[6]. Mitra SK, Dass N, Varshneya NC. Temperature dependence of the refractive index of water. J Chem Phys. 1972.57,1798-1799.

[7]. Abbate G., Bernini U., Ragozzino E., et al. The temperature dependence of the refractive index of water. J Phys D. 1978,11,1167-1172.

[8]. Weiss L., Tazibt A., Tidu, A., et al. Water density and polarizability deduced from the refractive index determined by interferometric measurements up to $250 \mathrm{MPa}$. JCP. 2012, 136, 124201.

[9]. Croccolo F., Plantier F., Galliero G., et al. Note: Temperature derivative of the refractive index of binary mixtures measured by using a new thermodiffusion cell. RSI. 2011, 82,126105 .

[10]. Yamamoto, I., Akira, I., Kanaga, A. Measurement of separative characteristics of H2HT Isotope separation using thermal diffusion column. J. Nucl. Sci. Technol. 1988,25,704711.

[11]. Naturforsch, C. Thermal diffusion as a mechanism for biological transport. Biosciences. 1984,39,656-661. 
[12]. Torres, JF., Komiya, A., Henry, D.; Maruyama, S. Measurement of Soret and Fickian diffusion coefficients by orthogonal phase-shifting interferometry and its application to protein aqueous solutions. J Chem Phys. 2013,139, 074203-1.

[13]. Mialdun, A., Shevtsova, V. Development of optical digital interferometry technique for measurement of thermodiffusion coefficients. Int J Heat Mass Transf. 2008,51,3164-3178.

[14]. Mialdun, A., Shevtsova, V. Measurement of the Soret and diffusion coefficients for benchmark binary mixtures by means of digital interferometry. J Chem Phys. 2011,134,044524-1.

[15]. Rahman, MA., Galand, Q., Soliman, M., Van Vaerenbergh, S., Saghir, MZ. Measurement of refractive indices of binary mixtures using digital interferometry and multi-wavelength abbemat refractometer. Optics and Lasers in Engineering. 2013,51,503-513.

[16]. Tasic, A., Djordjevic, D., Grozdanic, K., Radojkovic N. Use of mixing rules in predicting refractive indexes and specific refractivities for some binary liquid mixtures. J. Chem. Eng. Data. 1992,37,310-313.

[17]. Sechenyh, V. Legros, J., Shevtsova V. Experimental and predicted refractive index properties in ternary mixtures of associated liquids. J Chem Thermodyn. 2011,43,17001707.

[18]. Xu, Y., Yao, J., Wang, C., Li, H. Density, viscosity, and refractive index properties for the binary mixtures of $\mathrm{n}$-butylammonium acetate ionic liquid + alkanols at sveral temperatures. J Chem Eng Data. 2012,57,298-308.

[19]. Leahy-Dios, A., Bou-Ali, MM., Platten, JK., Firoozabadi, A. Measurements of molecular and thermal diffusion coefficients in ternary mixtures. J Chem Phys. 2005,122, 234502-1.

[20]. Koniger, A., Meiery, B., Kohler, W. Measurement of the Soret, diffusion, and thermal diffusion coefficients of three binary organic benchmark mixtures and of ethanol-water mixtures using a beam deflection technique. Philosophical Magazine. 2009,89,907-923.

[21]. Gebhardt, M., Köhler, W., Mialdun, A., Yasnou, V., Shevtsova, V. Diffusion, thermal diffusion, and Soret coefficients and optical contrast factors of the binary mixtures of dodecane, isobutylbenzene, and 1,2,3,4-tetrahydronaphthalene. J Chem Phys. $2013,138,114503$. 
[22]. Croccolo F., Bataller H., Scheffold F. A light scattering study of non-equilibrium fluctuations in liquid mixtures to measure the soret and mass diffusion coefficient. J Chem Phys. 2012,137,234202-1.

[23]. Wiegand, S., Ning, H., Kriegs, H. Thermal lens measurement of the Soret coefficient in acetone/water mixtures. Physical Chemisrty B. 2007,111,14169-14174.

[24]. Ning, H., Wiegand, S. Experimental investigation of the Soret effect in acetone/water and dimethylsulfoxide/water mixtures. J Chem Phys. 2006,125,221102-221104.

[25]. Köhler, W. Thermodiffusion in polymer solutions as observed by forced rayleigh scattering. J Chem Phys. 1993;98,660-668.

[26]. Fuji T., Arakawa M., Hattori T., Nakatsuka H. A white-light Michelson interferometer in the visible and near infrared regions. Rev Sci Instrum. 1998, 69(8), 2854-2858.

[27]. Ullmann V., Emam S., Manske E. White-light interferometers with polarizing optics for length measurements with an applicable zero-point detection. Meas. Sci. Technol. 2015, 26,084010 .

[28]. Xinhong Li, Yamauchi T., Iwai H., Yamashita Y. Full-field quantitative phase imaging by white-light interferometry with active phase stabilization and its application to biological samples. Opt Lett. 2006, 31(12), 1830-1832.

[29]. Liang Yi., Grover C. Modified white-light Mach-Zehnder interferometer for direct groupdelay measurements. Applied Opt. 1998,37(19),4105-4111.

[30]. Georis, Ph.; Montel, F.; Van Vaerenbergh, S.; Decroly, Y.; Legros, C. Measurement of the Soret coefficient in crude oil. Proceedings of the European Petroleum Conference. 1998,1,57-62.

[31]. Shevtsova V., Mialdun A., Melnikov D., Ryzhkov I., Gaponenko Y., Saghir MZ., Lyubimova T., Legros J. The IVIDIL experiment onboard the ISS: Thermodiffusion in the presence of controlled vibrations. Comptes Rendus - Mecanique. 2011,339,310-317.

[32]. Mialdun A, Yasnou V., Shevtsova V., et al. A comprehensive study of diffusion, thermodiffusion, and soret coefficients of water-isopropanol mixtures. J Chem Phys. 2012,136, 244512.

[33]. Mialdun A, Minetti C., Gapnenko, Shevtsova V., Dubois F. Analysis of the Thermal Performance of SODI Instrument for DCMIX Configuration. Microgravity Sci. Technol. 2013, 25,83-94. 
[34]. Shevtsova, V., Sechenyh, V, Nepomnyashchy, A., Legros J. Analysis of the application of optical two-wavelength techniques to measurement of the Soret coefficients in ternary mixtures. Philos Mag. 2011,91,3498-3518.

[35]. Platten J., Bou-Ali M., Costesèque P., Dutrieux J., Köhler W., Leppla C., Wiegand S., Wittko G. Benchmark values for the Soret, thermal diffusion and diffusion coefficients of three binary organic liquid mixtures.Philosophcal Mag. 2003, 83(17-18), 1965-1971.

[36]. Touriño, A., Hervello, M.; Moreno, V., Marino, G., Iglesias, M. Changes of refractive indices in ternary mixtures containing chlorobenzene $+\mathrm{n}$-hexane + (n-heptane or n-octane) at 298.15 K. J Serb Chem Soc. 2004,69,461-475.

[37]. Yassien M. Comparative study on determining the refractive index profile of polypropylene fibres using fast fourier transform and phase-shifting interferometry. J Opt A Pure Appl Opt. 2009,11, 075701.

[38]. De Graaff V. Human Anatomy, 4th ed. Wm. C. Brown Publishers, Dubuque, IO, USA, 1995.

[39]. Meinke M. Geronde I. Friebel M. Helfmann J. Muller G. Chemometric determination of blood parameters using visible-near-infrared spectra. Appl. Spectroscp. 2006, 559, 826-835.

[40]. Sklar L. Flow cytometry for biotechnology. Oxford university press. 2005.

[41]. Amer, M., Elnasser, T.A., El Haggar, S., Abdel-Malak, G., Zohdy, W. May-GrünwaldGiemsa stain for detection of spermatogenic cells in the ejaculate: A simple predictive parameter for successful testicular sperm retrieval. Hum Reprod. 2001, 16(17), 1427-1432.

[42]. Lewis S., Bain B., Bates I., Lewis D. Blood cell morphology in health and disease: practical hematology. Elsevier. 2006, 80-103.

[43]. Dariusz F. Detection of Erythrocyte Cells in Microscopy Images, West Pomeranian University of Technology, Szczecin, ISSN 0033-2097, R. 88 NR 10b/2012.

[44]. Lue N., Choi W., Popescu G., Ikeda T., Dasari R., Badizadegan K., Feld M., Quantitative phase imaging of live cells using fast Fourier phase microscopy, Appl. Opt. 2007,46,18361842.

[45]. Rappaz, B., P. Marquet, E. Cuche, Y. Emery, C. Depeursinge, and P.J. Magistretti.. Measurement of the integral refractive index and dynamic cell morphometry of living cells with digital holographic microscopy. Opt Express. 2005 13(23), 9361-9373. 
[46]. Marquet P., Rappaz B., Magistretti P., Cuche E., Emery Y., Colomb T., Depeursinge C. Digital holographic microscopy: a noninvasive contrast imaging technique allowing quantitative visualization of living cells with subwavelength axial accuracy, Opt. Lett. 2005, $30,468-470$.

[47]. Kemper B., Von Bally G. Digital holographic microscopy for live cell applications and technical inspection. Appl. Opt. 2008, 47, A52-A61.

[48]. Kühn, J., Montfort F., Colomb T., Rappaz B., Moratal C., Pavillon N., Marquet P., Depeursinge C. Submicrometer tomography of cells by multiple-wavelength digital holographic microscopy in reflection. Opt. Lett. 2009, 34, 653-655.

[49]. Rappaz B. Marquet P., Cuche E., Emery Y., Depeursinge C., Magistretti J. Measurement of the Integral refractive Index and Dynamic Cell Morphometry of Living Cells with Digital Holographic Microscopy. Opt Express. 2005,13(23), 9361-9373.

[50]. Ikeda T., Popescu G., Dasari R., Feld and M. Hilbert phase microscopy for investigating fast dynamics in transparent systems.Opt Lett. 2005,30, 1165-1167.

[51]. Lue N., Choi W., Popescu G., Yaqoob Z., Badizadegan K., Dasari R., Feld, M. Live cell refractometry using Hilbert phase microscopy and confocal reflectance microscopy. J. Phys. Chem. A 2009, 113, 13327-13330.

[52]. Popescu G., Ikeda T., Dasari R., Feld M. Diffraction phase microscopy for quantifying cell structure and dynamics. Opt Lett. 2006, 31, 775-777.

[53]. Choi W., Fang-Yen C., Badizadegan K., Oh S., Lue N., Dasari R., Feld M. Tomographic Phase Microscopy. Nat Methods. 2007, 4, 717-719.

[54]. Debnath S., Kothiyal P. Experimental study of the phase-shift miscalibration error in phaseshifting interferometry: use of a spectrally resolved white-light interferometer. Appl Optics. 2007, 46(22),103-5109.

[55]. Stewart B. Wild C. Wourld cancer report. ISBN. 2014, 978-92-832-0429-9.

[56]. Jemal A., Bray F., Center M., Ferlay J., Ward E., Forman D. Global Cancer Statistics. Cancer J. 2011, 61(2)69-90.

[57]. Königer A., Wunderlich H., Köhler W. Measurement of diffusion and thermal diffusion in ternary fluid mixtures using a two-color optical beam deflection technique. J Chem Phys. 2010,132, 174506. 
[58]. Seshasai S., Saghir M. Z. Experimental approaches to study thermodiffusion - A review. Int J Therm Sci. 2011,50,1125-1137.

[59]. Born, M., Wolf, E. Principles of Optics: Electromagnetic Theory of Propagation, Interference, and Diffraction of Light, 7th ed. Cambridge, England: Cambridge University Press, 1999.

[60]. Malacara D., Servín M., Malacara Z. Interferogram analysis for optical testing. Taylor \& Francis, Boca Raton, FL, 2005.

[61]. Baldwin E., Haniff A. The application of interferometry to optical astronomical imaging. Phil. Trans. 2001, A 360, 969-986.

[62]. Aleksoff, C. Multi-wavelength digital holographic metrology. Proc. SPIE. 2006, 6311, 63111D.

[63]. Little E., Finlay J. Strain measurement in biomechanics. Springer. 1992.

[64]. Lee C., Sheu W., Jaing C. An apparatus for the measurement of internal stress and thermal expansion coefficient of metal oxide films. Rev. Sci. Instrum. 2001, 72, 2128-2133.

[65]. Nguyen T., Wereley T. Fundamentals and applications of microfluidics. Artech House, Boston, MA, 2002.

[66]. Dyson, J. Interferometry as a Measurement Tool. Hunt Barnard Printing Ltd., Aylesbury, Great Britain, 1970.

[67]. Newport Corporation. Projects in Interferometry. Newport Corporation, Irvine, Ca, Revision: B1, 1993.

[68]. Francon, M. Optical Interferometry. Academic Press, New York and London 1966.

[69]. Wolf, E., Born, M. Principles of Optics. Oxford: Pregamon, 6th edition, 1983.

[70]. Goldberg KA, Bokor J. Fourier-transform method of phase-shift determination. Appl Opt. 2001, 40(17), 2886-94.

[71]. Quan C, Tay C J, Shang H., Bryanston-Cross J. Contour measurement by fibre optic fringe projection and Fourier transform analysis. Opt. Commun. 1995118 479-483.

[72]. Judge T., Bryanston-Cross P. A Review of Phase Unwrapping Techniques in Fringe Analysis. Opt. Laser Technol. 1994, 21, 199-239.

[73]. Gao P., Yao B., Lindlein N., Mantel K., Harder I., Geis E. Phase-shift extraction for generalized phase-shifting interferometry. Optics Letters. 2009, 34(22), 3553-3555. 
[74]. Vargas J., Quiroga A., Belenguer T. Phase-shifting interferometry based on principal component analysis. Optics Letters. 2011, 36(8), 1326-1328.

[75]. Chen L-C., Yeh S-L., Tapilouw A., Chang J-C. 3-D surface profilometry using simultaneous phase-shifting interferometry. Optics Commun. 2010, 283,3376-3382.

[76]. Langoju R., Patil A., Rastogi P. Super-resolution Fourier transform method in phase shifting interferometry. Opt Express. 2005,13, 7160-7173.

[77]. Zicha D., Dunn G., An image-processing system for cell behavior studies in subconfluent cultures, J. Microsc. 1995,179,11-21.

[78]. Popescu, G. Quantitative Phase Imaging of Cells and Tissues; McGraw-Hill Professional: New York, NY, USA, 2011.

[79]. Lue N., Choi W., Popescu G., Ikeda T., Dasari R., Badizadegan K., S. Feld M. Quantitative phase imaging of live cells using fast Fourier phase microscopy. Appl Optics. 2007,46,18361842.

[80]. Takeda M., Ina H., Kobayashi S. Fourier-transform method of fringe-pattern analysis for computer-based topography and interferometry. J Opt Soc Am .1982. 72,156-160.

[81]. Kreis T 1996 Holographic Interferometry (Berlin: Akademie).

[82]. (67). De Groot, S. R., Mazur, P. Non-equilibrium thermodynamics. Dover, New York; 1953.

[83]. Platten, JK., The Soret effect: A review of recent experimental results. J Appl Mech Trans ASME. 2006,73,5-15.

[84]. Rahman, MA., Saghir, MZ. Thermodiffusion or Soret effect: Historical review. Int. J. Heat Mass Transf. 2014,73,693-705.

[85]. Shukla, K., Kumar, A., Awasthi, N.; Srivastava, U., Gangwar, S. Density, viscosity and refractive index of binary liquid mixtures at 293.15, 298.15, 303.15, 308.15 and 313.15K. Exp Therm Fluid Sci. 2012,37,1-11.

[86]. Iulian, O., Ştefaniu, A., Ciocîrlan, O.; Croitoru, O. Property prediction for binary and ternary systems with water, 1,4-dioxane and ethylene glycol. II. refractive index. Rev Roumaine Chim. 2010,55,475-480.

[87]. Mehra, R. Application of refractive index mixing rules in binary systems of hexadecane and heptadecane with n-alkanols at different temperatures. Proc Indian Acad Sci Chem Sci. 2003,115,147-154. 
[88]. Wolf, B., Krotzsch, G. Geometry and dynamics in refracting systems. European Journal of Physics. 1995,16,14-20.

[89]. Tekin, N., Tarimci C. Study of the structure and refractive parameters of diethylamine and triethylamine. Opt Laser Tech. 2006,38,498-505.

[90]. Atik, Z. Experimental and predicted volumetric and refractive index properties of ternary mixtures of iodoethane with toluene and alcohols at temperature $298.15 \mathrm{~K}$ and pressure 101 kPa. J Chem Thermodyn. 2006,38,201-208.

[91]. Wittko G., Köhler W. Precise determination of the soret, thermal diffusion and mass diffusion coefficients of binary mixtures of dodecane, Isobutylbenzene and 1,2,3,4tetrahydronaphthalene by a holographic grating technique. Philos Mag. 2003.83,1973-1987.

[92]. Dubey GP., Tripathi N., Bhatia SC. Refractive index of ternary liquid systems of squalane (+ hexane + benzene; + cyclohexane + benzene and + hexane + cyclohexane). Indian J Pure Appl Phys. 2005,43,175-179.

[93]. Bhatti S., Prabhakar P., Singh P. Relative validity of refractive index mixing rules for ternary liquid mixtures. Indian Journal of Pure \& Applied Physics. 1995,33,18-22.

[94]. Yahya M. Saghir M.Z. Prediction and experimental measurement of refractive index in ternary hydrocarbon mixtures. J. Chem. Eng. Data, 2015, 60(8), 2329-2342.

[95]. Galand Q. Experimental investigation of the diffusive properties of ternary liquid systems. Ecole Polytechnique De Bruxelles, PhD dissertation; 2012.

[96]. Yousefi T., Ashjaee M. Experimental study of natural convection heat transfer from vertical array of isothermal horizontal elliptic cylinders. Exp Therm Fluid Sci. 2007,32,240248.

[97]. Perry E., McKelvie J. A comparison of phase shifting and Fourier methods in the analysis of discontinuous fringe patterns. Optics and Lasers in Engineering. 1993,19,269-284.

[98]. Kreis T. Digital holographic interference-phase measurement using the Fourier-transform method. Optics InfoBase. 1986,3,847-855.

[99]. Looyenga H. Dielectric constants of heterogeneous mixtures. Physicsa 1965, 31, 401-406.

[100]. Boulnois J.-L. Photophysical processes in recent medical laser developments: A review. Laser Med Sci. 1989, 1,47-66.

[101]. Carlton R. Pharmaceutical Microscopy. Springer New York. 2011, Chapter 2.

[102]. Yaroslavsky N., Priezzhev V., Rodriquez J., Yaroslavsky V., Battarbee H. Optics of 
blood. In Handbook of Optical Biomedical Diagnostics. SPIE-Press. 2002, 169-216.

[103]. Canham P., Anham P., Burton A. Distribution of size and shape in populations of normal red cells. Circulation Research. 1968, 422, 405-422.

[104]. Meinke M., Muller G., Friebel M., Helfmann J. Optical properties of platelets and blood plasma and their influence on the optical behavior of whole blood in the visible to near infrared wavelength range. J. Biomed. Opt. 2007,12,014024.

[105]. Meinke, C.; Friebel, M.; Helfmann, J. Optical Properties of Flowing Blood Cells. Advanced Optical Flow Cytometry: Methods and Disease Diagnoses. 2011, 95-132.

[106]. Jacques S. L. Optical properties of biological tissues: a review. Phys Med Biol. 2013,58, R37-61.

[107]. Kubelka P. New contributions to the optics of intensely light-scattering material. Part I. J Opt Soc. 1948, 38, 448-457.

[108]. Friebel M. Determination of optical properties of human blood in the spectral range 250 to $1100 \mathrm{~nm}$ using Monte Carlo simulations with hematocrit-dependent effective scattering phase functions. J Biomed Opt. 2006, 11(3), 034021.

[109]. Zhernovaya O., Sydoruk O., Tuchin V., Douplik A. The refractive index of human hemoglobin in the visible range. Phys. Med. Biol. 2011, 56, 4013-4021.

[110]. Roggan, A.; Friebel, M.; Dorschel, K.; Hahn, A.; Muller, G. Optical properties of circulating human blood in the wavelength range 400-2500 nm. J Biomed Opt.1999,436-46.

[111]. Yaroslavsky N., Yaroslavsky, V., Goldbach T., Schwarzmaier, H-J. Optical properties of blood in the near infrared spectral range. SPIE. 1996, 2678,314.

[112]. Hammer, M.; Schweitzer, D.; Michel, B.; Thamm, E.; Kolb. A Single scattering by red blood cells. Appl. Opt. 1998, 37, 7410 -7418.

[113]. Faber, D.; Aalers, M.; Mik, E.; Hooper B.; Leeuwen, T. Oxygen saturation-dependent absorption and scattering of blood. Phys. Rev. Lett. 2004, 93, 28102.

[114]. Sydoruk O., Zhernovaya O., Tuchin V., Douplik A. Refractive index of solutions of human hemoglobin from the near-infrared to the ultraviolet range: Kramers-Kronig analysis. J. Biomed. Opt. 2012,17,115002.

[115]. Jin Y., Chen J., Xu L., Wang P. Refractive index measurement for biomaterial samples by total internal reflection. Phys. Med. Biol. 2006,51, N371-N379.

[116]. Singh S. Refractive Index Measurement and its applications. Phys Scripta. 2002,65,167- 
180.

[117]. Friebel M., Meinke M. Model function to calculate the refractive index of native hemoglobin in the wavelength range of $250-1100 \mathrm{~nm}$ dependent on concentration. Appl Optics. 2006, 45(12), 2838-2842.

[118]. Barer R., Joseph S. Refractomery of living cells. Part I. Q J Micro Sec.1954, 95, 399-423.

[119]. Howard F. The determination of the refractive index of hemoglobin in solution. J. Biol. Chem.1920. 41, 537.

[120]. Hui Li, Lei Lin, Shusen Xie, Refractive index of human whole blood with different types in the visible and near-infrared ranges. SPIE. 2000, 3914.

[121]. Friebel M. Determination of the complex refractive index of highly concentrated hemoglobin solutions using transmittance and reflectance measurements. J Biomed Opt. 2005, 10(6), 064019.

[122]. Splinter R. Handbook of Physics in Medicine and Biology. Taylor and Francis Group. New York. 2010.

[123]. Splinter R. An Introduction to Biomedical Optics. Taylor and Francis Group. New York. 2007.

[124]. Shaked N., Satterwhite L., Telen M., Truskey G., Waxa A. Quantitative microscopy and nanoscopy of sickle red blood cells performed by wide field digital interferometry. J Biomed Opt. 2011, 16(3), 030506-1.

[125]. Cooke B., Mohandas N., Coppel R. The malaria-infected red blood cell: Structural and functional changes. 2001,50, 1-86.

[126]. Wile S., Sturgeon P. Thrombotic Thrombocytopenic Purpura: Review of the Subject with a Repori of Three Cases in Children. Pediatrics. 1956, 17(6):882-96.

[127]. Sullivan MT., Cotten R., Read EJ., Wallace EL. Blood collection and transfusion in the United States in 2001. Transfusion. 2007,47, 385-94.

[128]. Lee S., Gladwin T. Bad blood: the risks of red cell storage. Nat. Med. 2010, 16, 381-382.

[129]. D'Alessandro A., Liumbruno G., Grazzini G., Zolla L. Red blood cell storage: the story so far. Blood Transfus. 2010, 8, 82-88.

[130]. Cécile A., Alistair N., D Jamie C., Rinaldo B. Age of red blood cells and transfusion in critically ill patients. Annals of Intensive Care. 2013, 3, 1-11. 
[131]. Zimrin, B., Hess R. Current issues relating to the transfusion of stored red blood cells. Vox Sanguinis. 2009, 96, 93-103.

[132]. Orlov D., Karkouti K. The pathophysiology and consequences of red blood cell storage. Anaesthesia. 2015, 70, 29-37.

[133]. Bhaduri B., Kandel M., Brugnara C., Tangella K., Popescu G. Optical Assay of Erythrocyte Function in Banked Blood. Scientific Reports. 2015, 4, 6211.

[134]. Zubair C. Clinical impact of blood storage lesions. American Journal of Hematology. 2010, 85, 117-122.

[135]. Steiner ME., Stowell C. Does red blood cell storage affect clinical outcome? When in doubt, do the experiment.Transfusion 2009, 49, 1286-90.

[136]. Zolla L., Liumbruno G., D'Amici GM., Grazzini G. Adverse effects related to storage lesions: proteomic profiles of red blood cells during storage. Blood Transfus. 2009, s22-3.

[137]. Lelubre C., Piagnarelli, M., Vincent JL. Association between duration of storage of transfused red blood cells and morbidity and mortality in adult patients: myth or reality? Transfusion. 2009, 49, 1384-1394.

[138]. Gamé X., Soulié M., Fontanilles AM., Benoit JM., Corberand X., Plante P. Comparison of red blood cell volume distribution curves and phase-contrast microscopy in localization of the origin of hematuria. Urolory. 2003, 61, 507-511.,

[139]. Debnath, K., Park Y. Real-time quantitative phase imaging with a spatial phase-shifting algorithm. Opt. Lett. 2011, 36, 4677.

[140]. Wang Z., Millet L., Mir M., Ding H., Unarunotai S., Rogers J., Gillette M., Popescu G. Spatial light interference microscopy (SLIM). Opt Express. 2011, 19, 1016-1026.

[141]. Pham H., Bhaduri B., Tangela K., Popescu C., Popescu G. Real time blood testing using quantitative phase imaging. PLoS One. 2013, 8, e55676.

[142]. Anna T., Srivastava V., Mehta S. Shakher C. High-resolution full-field optical coherence microscopy using a Mirau interferometer for the quantitative imaging of biological cells. Appl Optics. 2011, 50, 6343-6351. 
[143]. Mehta S., Srivastava V. Quantitative phase imaging of human red blood cells using phaseshifting white light interference microscopy with colour fringe analysis. Appl Phys Lett. 2012, 101.

[144]. Jang Y., Jang J., Park Y. Dynamic spectroscopic phase microscopy for quantifying hemoglobin concentration and dynamic membrane fluctuation in red blood cells. Opt Express. 2012, 20, 9673-9681.

[145]. Wang Z., Tangella K., Balla A., Popescu G. Tissue Refractive Index as Marker of Disease. J Biomed Opt. 2011, 16, 116017-1160177.

[146]. Malacara D. Optical Shop Testing. John Wiley \& Sons, Inc., Hoboken, 3rd edition. 2007.

[147]. Goodman W. Introduction to Fourier Optics. New York: McGraw-Hill, 1996.

[148]. Ghiglia C., Pritt D. Two-Dimensional Phase Unwrapping: Theory, Algorithms, and Software (Wiley,1998).

[149]. Goldstein R., Zebker H., Werner C. Satellite radar interferometry: Two-dimensional phase unwrapping. Radio Sci.1988, 23, 713.

[150]. Mora-González M., Muñoz-Maciel J., Casillas F., Peña-Lecona, G. et al (2008). Canny, J. A Computational Approach to Edge Detection, IEEE Trans. on Pattern Analysis and Machine Intelligence. 1986, 8, 679-698.

[151]. Park K., Diez-Silva M., Popescu G., Lykotrafitis G., Choi W., Feld S., Suresh S. Refractive index maps and membrane dynamics of human red blood cells parasitized by Plasmodium falciparum. Proc. Natl. Acad. Sci. 2008, 105, 13730-13735.

[152]. Anand A., Chhaniwal K., Patel R., Javidi B. Automatic identification of malaria-infected RBC with digital holographic microscopy using correlation algorithms. IEEE Photonic J. 2012, 4, 1456-1464.

[153]. D’Avenio G., Caprari P., Daniele C., Tarzia A., Grigioni M. A software for analysing RBCs images under the flow. Computational modelling of objects represented in images. ISBN. 2012, 978-0-415-62134

[154]. Patil P., Sable G., Anandgaokar G. International Journal of Research in Engineering and Technology. eISSN, 2014, 3, 391-394.

[155]. Abbas N., Muhamad D., Abdullah A. Semi-Automatic clustered red blood cells splitting and counting in thin blood smear images. IRICT. 2014, 342-351. 